Utah State University

DigitalCommons@USU

$5-2015$

\title{
DNA Methylation in Lung Tissues of Mouse Offspring Exposed In utero to Polycyclic Aromatic Hydrocarbons
}

Trevor J. Fish

Utah State University

Follow this and additional works at: https://digitalcommons.usu.edu/etd

Part of the Biochemistry Commons

\section{Recommended Citation}

Fish, Trevor J., "DNA Methylation in Lung Tissues of Mouse Offspring Exposed In utero to Polycyclic Aromatic Hydrocarbons" (2015). All Graduate Theses and Dissertations. 4578.

https://digitalcommons.usu.edu/etd/4578

This Thesis is brought to you for free and open access by the Graduate Studies at DigitalCommons@USU. It has been accepted for inclusion in All Graduate Theses and Dissertations by an authorized administrator of DigitalCommons@USU. For more information, please contact digitalcommons@usu.edu.

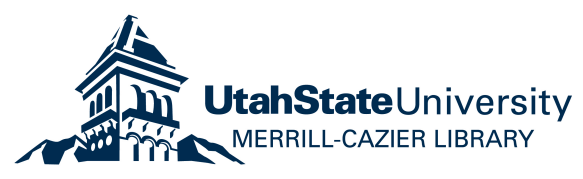


DNA METHYLATION IN LUNG TISSUES OF MOUSE OFFSPRING EXPOSED

IN UTERO TO POLYCYCLIC AROMATIC HYDROCARBONS

by

Trevor J Fish

A thesis submitted in partial fulfillment

of the requirements for the degree

of

MASTER OF SCIENCE

in

Toxicology

Approved:

Abby D. Benninghoff, Ph.D.

Major Professor

Roger A. Coulombe Jr., Ph.D.

Committee Member

Korry J. Hintze, Ph.D.

Committee Member
Aaron L. Olsen, D.V.M., Ph.D.

Committee Member

S. Clay Isom, Ph.D.

Committee Member

Dirk K. Vanderwall, D.V.M., Ph.D. ADVS Department Head

Mark R. McLellan, Ph.D.

Vice President for Research and

Dean of the School of Graduate Studies

UTAH STATE UNIVERSITY

Logan, Utah 
Copyright (C) Trevor J Fish 2015

All Rights Reserved 


\author{
ABSTRACT \\ In Utero to Polycyclic Aromatic Hydrocarbons \\ by \\ Trevor J Fish, Master of Science \\ Utah State University, 2015
}

DNA Methylation in Lung Tissues of Mouse Offspring Exposed

Major Professor: Dr. Abby D. Benninghoff

Department: Animal, Dairy and Veterinary Sciences

Polycyclic aromatic hydrocarbons (PAHs) comprise an important class of environmental pollutants that are known to cause lung cancer in animals and are suspected lung carcinogens in humans. PAHs are also known to cause cancer in offspring when provided to a pregnant mouse. Moreover, evidence from cell-based studies points to PAHs as modulators of the epigenome, that is modifications to DNA structure that control the expression of genes. The objective of this thesis research was to determine the impact of transplacental exposure of PAHs on the epigenome of fetal and adult lung tissues in offspring exposed to either dibenzo[def, $p]$ chrysene (DBC) or benzo $[a]$ pyrene (BaP) throughout gestation and lactation. Lung tumor incidence in 45-week old mice initiated with $\mathrm{BaP}$ was $30 \%$, much lower than that of the $\mathrm{DBC}$-exposed offspring at $100 \%$. The spontaneous lung tumor rate was $9 \%$ in control offspring at 45 -weeks. Promoter methylation for Cdkn2a, Rarb, Dapk1 and Mgmt genes was assessed by 
bisulfite sequencing. Laser capture microdissection was employed to assess the methylation of these selected tumor suppressor genes during the process of tumor progression. Analysis of methylation for the $C d k n 2 a$ promoter revealed a localized region of moderate methylation associated with $\mathrm{CpG}$ sites 27 to 30 in all samples evaluated. No differences in methylation were apparent for the other target genes. Genome-wide methylation of lung tumors in adult offspring was determined using the NimbleGen DNA Methylation array. The genome-wide assessment of $\mathrm{CpG}$ island promoter methylation revealed distinct patterns of methylation associated with normal and adenocarcinoma lung tissues. Furthermore, gene ontology analysis revealed differences in the biological functions represented by the methylated genes in normal lung tissues compared to tumor tissues. Altogether, the research presented here identified several new target genes of interest for future studies investigating the epigenetics of PAH-initiated lung cancer. This work also provided new knowledge that transplacental exposure to PAHs can lead to distinct DNA methylation profiles in lung tumors in adult offspring. 


\title{
PUBLIC ABSTRACT
}

\author{
DNA Methylation in Lung Tissues of Mouse Offspring Exposed \\ In Utero to Polycyclic Aromatic Hydrocarbons
}

by

\author{
Trevor J Fish, Master of Science \\ Utah State University, 2015
}

\begin{abstract}
Major Professor: Dr. Abby D. Benninghoff
Department: Animal, Dairy and Veterinary Sciences
\end{abstract}

Polycyclic aromatic hydrocarbons (PAHs) comprise an important class of environmental pollutants that are known to cause lung cancer in animals and suspected lung carcinogens in humans. PAHs are also known to cause cancer in offspring when provided to a pregnant mouse. Some evidence from cell-based studies points to PAHs as modulators of the epigenome, that is modifications to DNA structure that control the expression of genes. Inappropriate changes to the epigenome and consequently expression of cancer-critical genes are often characteristic of cancer cells. The objective of this thesis research was to determine the impact of transplacental exposure to two model PAHs on the epigenome of fetal and adult lung tissues in offspring. Specifically, we measured patterns of methylation of DNA, a type of epigenetic mark, in different types of lung tissue to assess changes in the epigenome associated with development of lung cancer. Two strategies were employed: 1) a targeted approach using ultra-deep bisulfite sequencing to precisely measure the specific pattern of methylated sites in the 
promoter regulatory region for several tumor suppressor genes, including Cdkn2a, Rarb, Dapk1 and Mgmt; and 2) a broad, genome-wide approach using a microarray covering all regulatory promoter regions in the entire mouse genome. Our first approach did not yield any marked differences in methylation patterns for any of the target genes for lung tissues obtained at birth or at various ages up to 45 weeks, nor according to the type of tissue (normal, pre-neoplastic, tumor). However, the genome-wide approach did yield specific patterns of methylation in lung tumors, including distinct profiles associated with lung tumor tissue from PAH-exposed animals that were substantially different from normal lung tissue in control animals. Altogether, the research presented here identified several new target genes of interest for future studies investigating the epigenetics of PAHinitiated lung cancer. This work also provided new knowledge that exposure to PAHs can lead to distinct DNA methylation profiles in lung tumors in adult offspring. 


\section{ACKNOWLEDGMENTS}

I would like to thank my advisor, Dr. Abby Benninghoff, who has mentored, supported and encouraged me throughout my studies at Utah State University. It was due to her enthusiasm and passion for science that I began my own journey into the realm of scientific research. I also thank my committee members, Dr. Roger Coulombe, Dr. Clay Isom, Dr. Aaron Olsen and Dr. Korry Hintze, for their support and assistance in my graduate work. I would like to thank my laboratory colleagues Deanna Larson, Stephany Perez and Amanda Hagman for always being willing to lend a helping hand.

I especially am grateful to my wife Callie and my daughter Chloe for their love, support and patience as I pursued this goal. I would not have been able to complete this graduate program without them.

This work was supported by the National Institute of Environmental Health and Safety (grant number RO3ES018919) and the Utah Agriculture Experiment Station (project number UTA00172). 
CONTENTS

Page

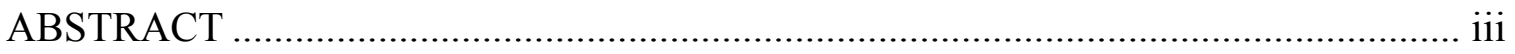

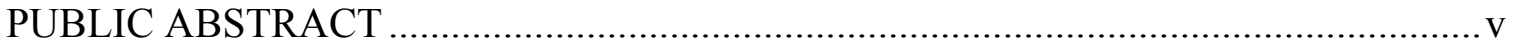

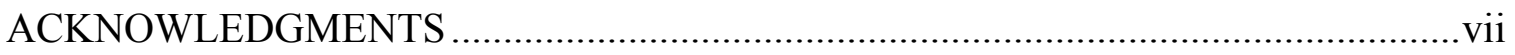

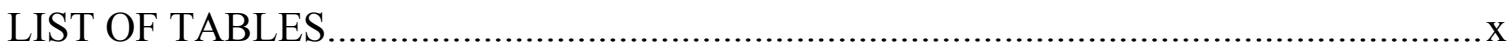

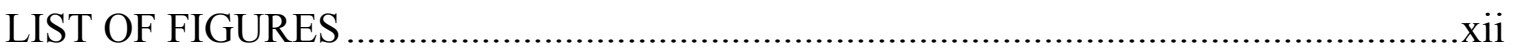

LIST OF ABBREVIATIONS AND SYMBOLS .........................................................

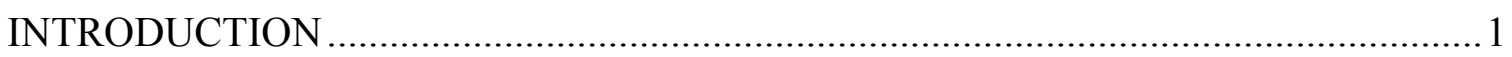

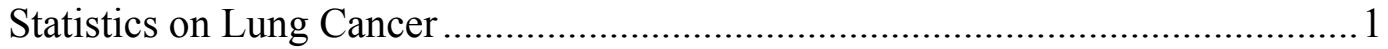

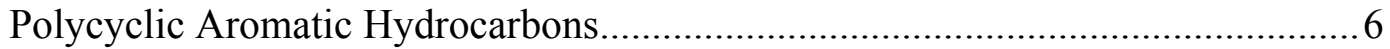

Epigenetics and Lung Cancer ........................................................................... 16

Project Objectives and Hypothesis ................................................................2 21

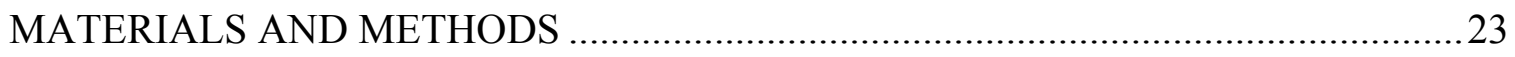

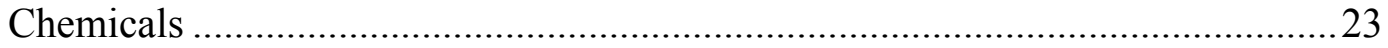

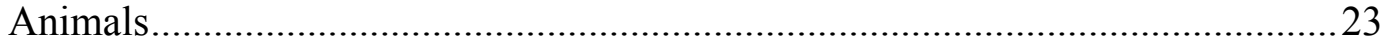

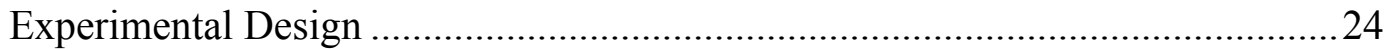

Lung Tissue Collection and Processing...........................................................25

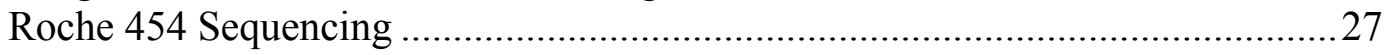

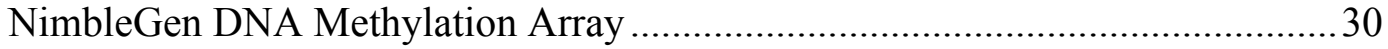

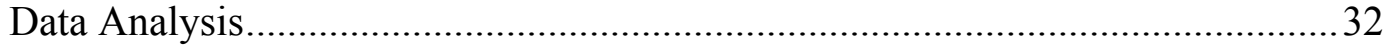

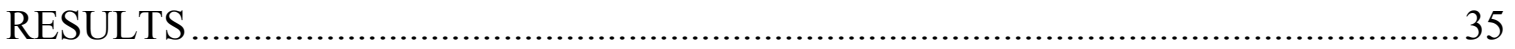

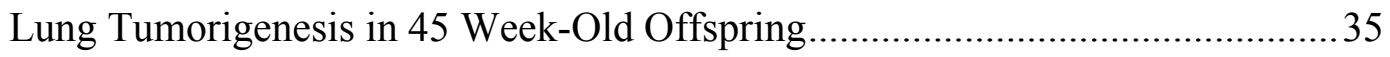

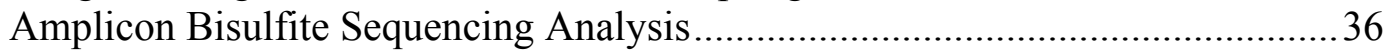

Genome-wide Methylation Analysis ........................................................ 46

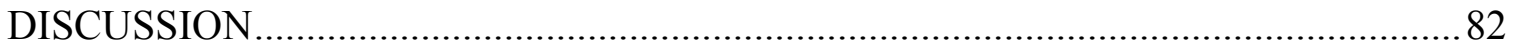

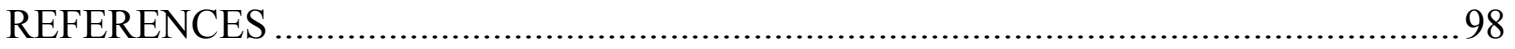

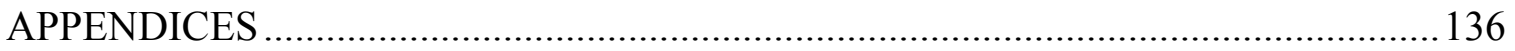

Appendix A: NimbleGen Processed Data Report for All Methylated

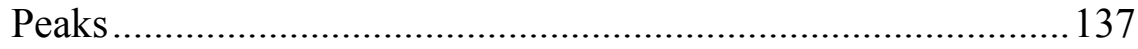

Appendix B: NimbleGen Processed Data Report for Nearest

Methylated Peaks. 
Appendix C: Peak Scores Summary Table for All Methylated Genes in Any Data Set....................................................................... 141

Appendix D: Gene Lists for Ontology Analyses.......................................... 142

Appendix E: Results of Gene Ontology Analyses for Differentially Methylated Genes ....................................................................... 143

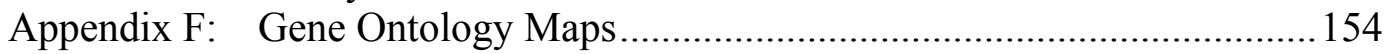




\section{LIST OF TABLES}

Table

1 Select Genes Altered by Hypermethylation in Lung Cancer

2 Primers Used for Bisulfite Sequencing . 28

3 Average Methylation per CpG Site for the Cdkn2a Promoter Region Sequenced by Roche 454.

4 Average Methylation per CpG Site for the Dapk1 Promoter Region

Sequenced by Roche 454

5 Average Methylation per CpG Site for the Mgmt Promoter Region Sequenced by Roche 454.

6 Average Methylation per CpG Site for the Rarb Promoter Region Sequenced by Roche 454 .

$7 \quad$ Functional Analysis of Differently Methylated Genes for Adjacent and Tumor Tissues of Lungs From DBC-Initiated Mice and From Normal Lung Tissue of Sham-Initiated Mice

$8 \quad$ Functional Analysis of Differently Methylated Genes for Adjacent and Tumor Tissues of Lungs From BaP-Initiated Mice and From Normal Lung Tissue of Sham-Initiated Mice

$9 \quad$ Functional Analysis of Differently Methylated Genes for Tumor Tissues Obtained From Lungs of DBC- or BaP-Initiated Mice and From Normal Lung Tissue of Sham-Initiated Mice

A.1 List of File Names Included in the Archive File for Appendix A ...................... 137

A.2 Definitions of File Contents for Appendix A by Spreadsheet Column Title ...... 138

B.1 List of File Names Included in the Archive File for Appendix B ..................... 139

B.2 Definitions of File Contents for Appendix B by Spreadsheet Column Title....... 140

C.1. Definitions of File Contents or Appendix C by Spreadsheet Column Title........ 141

D.1 List of Comparisons Among Tissue Types to Generate Gene Lists for Ontology Analyses..... 
E.1 AgriGO GO Slim Results for Biological Process, Molecular Function and Cellular Compartment for Sham, AdjDBC and TumDBC Tissues

E.2 AgriGO GO Slim Results for Biological Process, Molecular Function and Cellular Compartment for Sham, AdjBaP and TumBaP Tissues

E.3 AgriGO GO Slim Results for Biological Process, Molecular Function and Cellular Compartment for sham, TumDBC and TumBaP Tissues

F.1 List of File Names Included in the Archive File for Appendix F. 


\section{LIST OF FIGURES}

Figure $\quad$ Page

$1 \quad$ Worldwide lung cancer incidence and mortality ........................................... 1

2 Chemical structures for example polycyclic aromatic hydrocarbons.................... 6

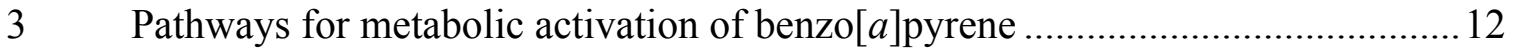

4 Pathways for metabolic activation of dibenzo[def,p]chrysene ........................... 14

5 Representative microscopy images of mouse lung tissues collected for laser capture microdissection ................................................................... 25

6 Location of primer sequences with respect to predicted $\mathrm{CpG}$ islands

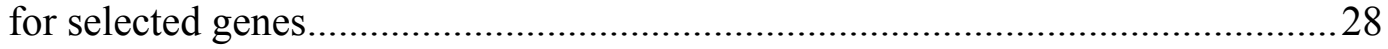

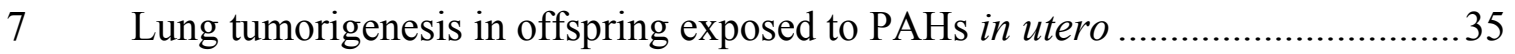

8 Methylation profile for region of $C d k n 2 a$ gene promoter by 454 deep bisulfite sequencing

$9 \quad$ Methylation profile for region of Dapk1 gene promoter by 454 deep bisulfite sequencing

10 Methylation profile for region of Mgmt gene promoter by 454 deep

bisulfite sequencing

11 Methylation profile for region of Rarb gene promoter by 454 deep bisulfite sequencing

12 Venn diagrams depicting segregation of differentially methylated genes among lung tissue types

13 Biological processes associated with genes differentially methylated in lung tumors from both $\mathrm{DBC}$ - and $\mathrm{BaP}$ initiated offspring

14 Biological processes associated with genes differentially methylated in normal lung tissue from sham-initiated offspring

15 Biological processes associated with genes differentially methylated in lung tumor for either DBC- or BaP initiated offspring

16 Methylation of Bmpl promoter in normal and tumor lung tissues .65

17 Methylation of Fgfrlop promoter in normal and tumor lung tissues 66 
18 Methylation of Hoxbl promoter in normal and tumor lung tissues

19 Methylation of $B c l 2 l 11$ promoter in normal and tumor lung tissues...................69

20 Methylation of $P d c d 4$ promoter in normal and tumor lung tissues ......................70

21 Methylation of Pten promoter in normal and tumor lung tissues....................... 71

22 Methylation of Casp7 promoter in normal and tumor lung tissues ..................... 72

23 Methylation of $1 l 11$ promoter in normal and tumor lung tissues........................ 73

24 Methylation of Maea promoter in normal and tumor lung tissues ...................... 75

25 Methylation of Tpd52l1 promoter in normal and tumor lung tissues...................76

26 Methylation of $C d k n 2 a$ promoter in normal and tumor lung tissues ...................77

27 Methylation of Dapklpromoter in normal and tumor lung tissues ......................78

28 Methylation of $\mathrm{Mgmt}$ promoter in normal and tumor lung tissues .................... 79

29 Methylation of Rarb promoter in normal and tumor lung tissues ...................... 81

F.1 GO Slim Map for Methylated in Sham, Not AdjDBC or TumDBC ................. 154

F.2 GO Slim Map for Methylated in AdjDBC, Not Sham or TumDBC .................. 154

F.3 GO Slim Map for Methylated in TumDBC, Not Sham or AdjDBC .................. 154

F.4 GO Slim Map for Methylated in Sham, Not AdjBaP or TumBaP ..................... 154

F.5 GO Slim Map for Methylated in AdjBaP, Not Sham or TumBaP ..................... 154

F.6 GO Slim Map for Methylated in TumBaP, Not Sham or AdjBaP ..................... 154

F.7 GO Slim Map for Methylated in Sham, Not TumDBC or TumBaP .................. 154

F.8 GO Slim Map for Methylated in TumDBC, Not Sham or TumBaP .................. 154

F.9 GO Slim Map for Methylated in TumBaP, Not Sham or TumDBC .................. 154

F.10 GO Slim Map for Methylated in TumDBC and TumBaP, Not Sham ............... 154 


\section{LIST OF ABBREVIATIONS AND SYMBOLS}

$\begin{array}{ll}\text { Abbreviations } & \\ \text { AAH } & \text { Atypical adenomatous hyperplasia } \\ \text { AdC } & \text { Adenocarcinoma } \\ \text { AhR } & \text { Aryl hydrocarbon receptor } \\ \text { ALK } & \text { Anaplastic lymphoma kinase } \\ \text { ANOVA } & \text { Analysis of Variance } \\ \text { ARNT } & \text { AhR nuclear translocator } \\ \text { BaP } & \text { Benzo[a]pyrene } \\ \text { Bcl2l11 } & \text { Bcl-2-like protein 11 } \\ \text { BISMA } & \text { Bisulfite Sequencing DNA Methylation Analysis } \\ \text { BMP } & \text { Bone morphogenetic proteins } \\ \text { bp } & \text { Base pair } \\ \text { Casp7 } & \text { Caspase-7 } \\ \text { CDC } & \text { Centers for Disease Control and Prevention } \\ \text { Cdkn2a } & \text { Cyclin-dependent kinase inhibitor 2a } \\ \text { CO } 2 & \text { Carbon dioxide } \\ \text { CS } & \text { Common sequence tag } \\ \text { CYP } & \text { Cytochrome P450 } \\ \text { dA } & \text { Deoxyadenosine } \\ \text { Dapk1 } & \text { Death-associated protein kinase } \\ \text { DBC } & \text { Dibenzo[def,p]chrysene } \\ \text { dG } & \text { Deoxyguanosine } \\ \text { DNMT } & \text { DNA methyltransferase } \\ \text { DOI } & \text { Digital Object Identifier } \\ \text { EGFR } & \text { Epidermal growth factor receptor } \\ \text { Fgfrlop } & \text { Fibroblast growth factor receptor 1 oncogene partner } \\ \text { GSH } & \text { Glutathione } \\ \text { GO } & \text { Gene Ontology } \\ \text { GST } & \text { Glutathione-s-transferases } \\ \text { HOX } & \text { Homeobox } \\ \text { Hoxb1 } & \text { Homeobox B1 } \\ \text { Ill1 } & \text { Interleukin 11 } \\ \text { IR } & \text { Infrared } \\ \text { KRAS } & \text { Kirsten rat sarcoma viral oncogene homolog } \\ \text { KS } & \text { Kolmogorov-Smirnov } \\ \text { LCM } & \text { Laser capture micro-dissection } \\ \text { Maea } & \text { Macrophage erythroblast attacher } \\ M g m t & \text { O'-methylguanine-DNA methyltransferase } \\ \text { MID } & \text { Multiplex identifier } \\ \text { NNK } & \text { 4-(methylnitrosamino)-1-(3-pyridyl)-1-butanone } \\ \text { NNN } & \text { N'-nitrosonornicotine } \\ \text { NSCLC } & \text { Non-small cell lung cancer } \\ & \\ & \\ \text { a } & \end{array}$




$\begin{array}{ll}\text { OCT } & \text { Optimal Cuttin } \\ \text { PAH } & \text { Polycyclic A } \\ \text { Pdcd4 } & \text { Programmed } \\ \text { PEN } & \text { Polyethylene } \\ \text { Pten } & \text { Phosphatase } \\ \text { Rarb } & \text { Retinoic acid } \\ \text { SEA } & \text { Singular enrich } \\ \text { SULT } & \text { Sulfotransfer } \\ \text { TP53 } & \text { Tumor prote } \\ \text { Tpd52ll } & \text { Tumor protein } \\ \text { UGT } & \text { UDP-glucoronc } \\ \text { WGA } & \text { Whole Geno } \\ \text { XRE } & \text { Xenobiotic resh } \\ \text { Symbols } & \\ \AA & \\ \text { C } & \text { Angstom } \\ \mathrm{kg} & \text { Celsius } \\ \mathrm{mg} & \text { Kilogram } \\ \mathrm{min} & \text { Milligram } \\ \mathrm{ml} & \text { Minute } \\ \mathrm{mm} & \text { Milliliter } \\ \mathrm{ng} & \text { Millimeter } \\ \mathrm{sec} & \text { Nanogram } \\ \mu \mathrm{g} & \text { Second } \\ \mu \mathrm{l} & \text { Microgram } \\ \mu \mathrm{m} & \text { Microliter } \\ \mu \mathrm{M} & \text { Micrometer } \\ & \text { Micromolar } \\ & \end{array}$




\section{INTRODUCTION}

\section{Statistics on Lung Cancer}

Lung cancer is the leading cause of cancer death worldwide and is the most common cancer in the world, with estimated deaths totaling $1,590,000$ in 2012 . New cases were estimated to be 1.8 million in $2012,58 \%$ of which occurred in economically less-developed regions of the world (Fig. 1) (Ferlay, et al., 2013). For the United States,

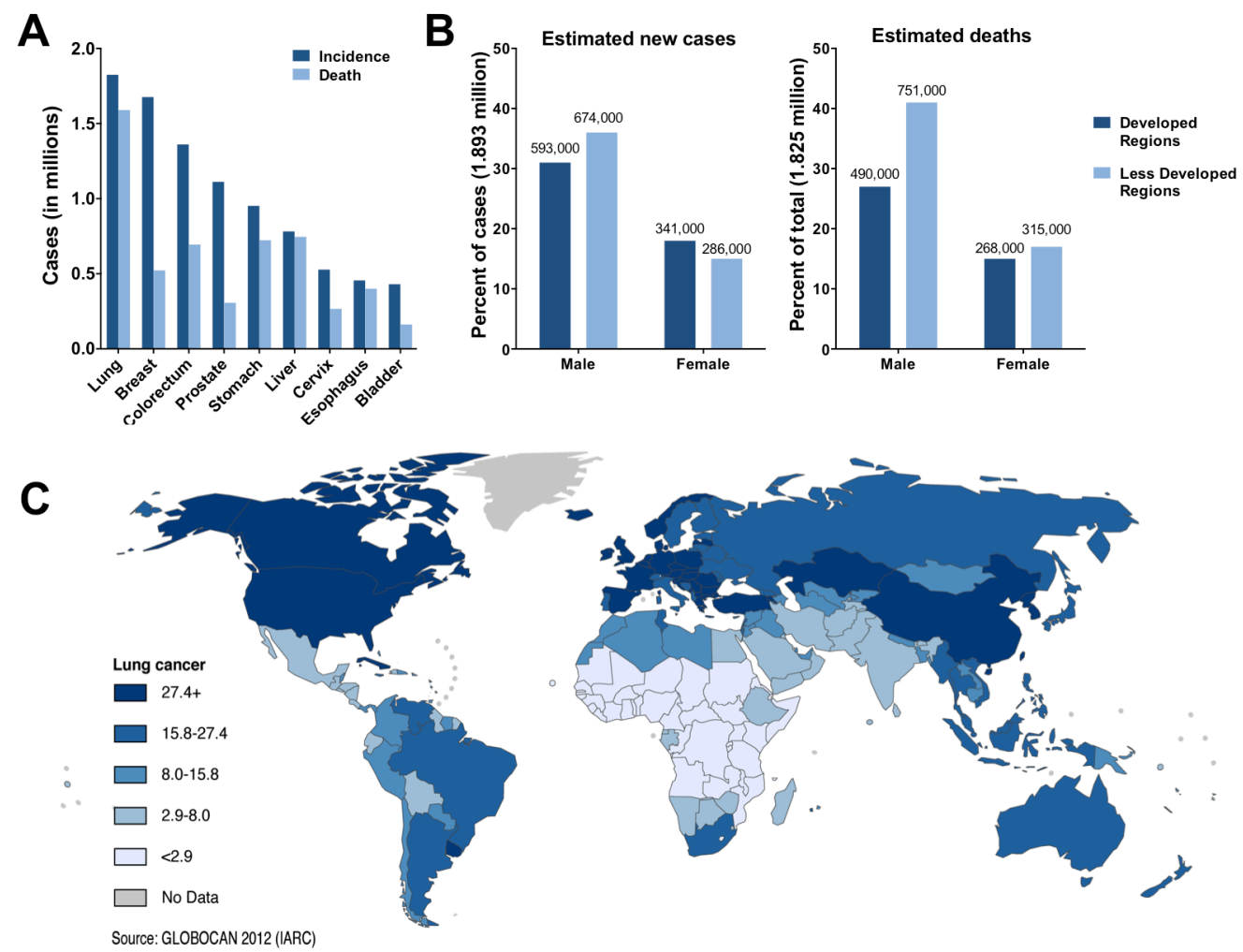

FIG. 1. Worldwide lung cancer incidence and mortality. (A) Estimated number of cases for the most common cancers worldwide in 2012. (B) Bars represent the estimated number of new lung cancer cases and the estimated number of lung cancer deaths as a percentage of all cancers (excluding non-melanoma skin cancers) for male or females in developed (dark blue) or developing (light blue) nations. Numbers above the bar represent the number of cases or deaths. (C) World map depicting incidence of lung cancer by nation for both males and females (values shown are the agestandardized rate per 100,000 people). Source data were obtained from the GLOBOCAN 2012 database (Ferlay, et al., 2013). 
an estimated 224,210 new cases of lung cancer were documented in 2014, a number that accounts for about $13 \%$ of all cancer diagnoses that year. The estimated new cases of lung cancer for both males and females rank second after prostate cancer for males and breast cancer for females. The rate of lung cancer incidence in men started to decline in the mid-1980s, whereas a decline was evident for women in the mid-2000s (American Cancer Society, 2015). Estimated deaths in 2014 due to lung cancer are higher than the combined total for prostate cancer, breast cancer, and colorectal cancer (Siegel et al., 2014). Lung cancer is a deadly disease with a poor five-year survival rate of only $17 \%$. In addition, roughly half of lung cancer patients die within one year of being diagnosed (Howlader N, 2015; Siegel, et al., 2014).

The two main types of lung cancer are small cell lung cancer (13\% of cases) and non-small cell lung cancer (83\%), with non-small cell lung cancer (NSCLC) being further classified within three main subtypes: adenocarcinoma, squamous-cell carcinoma and large-cell carcinoma (American Cancer Society, 2015). Adenocarcinoma is usually found in peripheral locations of the lung but also can be observed centrally in a main bronchus. Squamous-cell lung carcinoma tends to be found near the middle of the lungs, near a bronchus. Large-cell carcinoma can appear in any part of the lung.

Adenocarcinoma is the predominant form of lung cancer diagnosed in neversmokers, whereas smokers develop all major histological types of lung cancer (MacKinnon et al., 2010). The diagnosis and staging of lung cancer are often performed simultaneously, and some of the common tests and procedures used include physical exam, chest x-ray, CT scan, biopsy, sputum cytology and immunohistochemistry. 
NSCLC progresses through several stages as it develops from simple abnormal cells to full adenocarcinoma of the lung. Briefly, in stage 0, also called carcinoma in situ, abnormal cells are found in the lining of airways. In stage I patients, the cancer is localized to the lungs but has not spread to any lymph nodes outside the lung. Stage II is typified by spread of the cancer to nearby lymph nodes. Stage III is subdivided into IIIA and IIIB depending upon the location of the tumor and the infiltrated lymph nodes. Stage IV is the most advanced stage of lung cancer in which cancer has spread to both lungs and metastasized to other organs, such as the brain, liver, adrenal glands, kidneys or bone (National Cancer Institute, 2015). Metastases to these critical organs account for the majority of lung cancer deaths (Nichols et al., 2012).

Lung cancer incidence and mortality vary with respect to race. The Centers for Disease Control and Prevention (CDC) reported that among men, black men had the highest rates for lung cancer incidence and mortality, followed by white men and then by men representing other ethnic groups. Conversely, white women had the highest incidence and mortality rates, followed by black women (Centers for Disease Control and Prevention, 2014). As with many cancers, risk of developing lung cancer increases with age.

Today, lung cancer risk typically is greater in men than in women, and the higher risk apparent for men has been attributed to smoking habits, environment and lifestyle choices (Freedman et al., 2008; Sun et al., 2007). The issue of gender in lung cancer risk has been debated since the early 1990s, at which time reports from case control studies showed higher odds ratio in women than men (Osann et al., 1993; Risch et al., 1993; 
Zang and Wynder, 1996). Cohort studies consistently found higher lung cancer death rates in men than women, both in the presence (Thun et al., 2002) and absence (Thun et al., 2008; Thun et al., 2006) of smoking. However, this pattern did not appear to be consistent when considering newly diagnosed cases (Bach et al., 2003). Some researchers have hypothesized that, among smokers, women may have higher risk of developing lung cancer than men but lower risk of dying from the disease (Henschke and Miettinen, 2004; Henschke et al., 2006). Additionally, Wakelee, et al. (2007) documented that lung cancer incidence rates were higher in women than men among never-smokers. The accumulated evidence supports the notion that lung cancer risk is not consistent for men and women. Further research is needed to elucidate the gender differences in respect to lung cancer risk.

Lung cancer risk is also elevated among people with genetic susceptibility, such as the carriers of tumor protein 53 (TP53) germline sequence variations, often as a hereditary feature (Molina et al., 2008). The tumor suppressor gene TP53 plays a vital role in the process of apoptosis and is an important stress response molecule that is activated in response to diverse intrinsic and extrinsic signals (Rozan and El-Deiry, 2007). In addition, there is a linear relationship between TP53 mutation risk and tobacco consumption, as mutations of this gene are less frequent in never-smokers than in those who smoke (Hernandez-Boussard and Hainaut, 1998; Le Calvez et al., 2005). Increased lung cancer risk is also associated with mutations in other genes, including epidermal growth factor receptor $(E G F R)$, anaplastic lymphoma kinase $(A L K)$ and kirsten rat sarcoma viral oncogene homolog (KRAS) (Couraud et al., 2012; Pendharkar et al., 2013). 
$E G F R$ is responsible for a cascade of biological effects in normal cells and is often involved in cancer cell biology by activating tumorigenic processes, such as cell proliferation and angiogenesis (Schlessinger, 2002). $A L K$ is a receptor tyrosine kinase and the EML4 (echinoderm microtubule-associated protein-like 4)-ALK fusion is the most common $A L K$ rearrangement in NSCLC (Choi et al., 2008; Pendharkar, et al., 2013). This receptor has gained clinical relevance and importance as a therapeutic target for NSCLC. KRAS is involved primarily in regulating cell division as part of the RAS/mitogen-activated protein kinase (MAPK) signaling pathway. KRAS mutations are found in $20-30 \%$ of NSCLC cases, predominately in adenocarcinomas (Couraud, et al., 2012). Changes in these and other genes may make some lung cancers more likely to grow and metastasize.

Cigarette smoking is by far the most important risk factor for lung cancer. About $90 \%$ of lung cancer cases have been attributed to this behavior, and risk of lung cancer increases with both quantity and duration of smoking (Centers for Disease Control and Prevention, 2014). Besides smoking cigarettes, several other environmental risk factors may increase the chance of developing lung cancer. Exposure to radon gas released from soil and building materials is estimated to be the second leading cause of lung cancer in Europe and North America (American Cancer Society, 2015). Other risk factors include environmental or occupational exposure to asbestos, silica, certain metals (cadmium, nickel, chromium, arsenic), radiation, air pollution, soot, tar and diesel exhaust (American Cancer Society, 2015; Centers for Disease Control and Prevention, 2014; Molina, et al., 2008; Siegel, et al., 2014). Never-smokers account for 10-15\% of all lung 
cancer cases, and these are typically associated with combination of genetic factors and exposure to other forms of air pollution (Thun, et al., 2008). Some researchers regard lung cancer in never-smokers as a different disease, which would then be ranked as the seventh leading cause of cancer death worldwide (Sun, et al., 2007; Wakelee, et al., 2007).

Polycyclic Aromatic Hydrocarbons

Polycyclic Aromatic Hydrocarbons (PAHs) comprise a large class of structurally diverse organic molecules comprised of two or more fused aromatic rings (Fig. 2). PAHs are non-polar, lipophilic, organic compounds that do not contain any heteroatoms. Fully aromatic PAHs are generally planar with a molecular thickness of $3.7 \AA$ (Harvey, 1991).

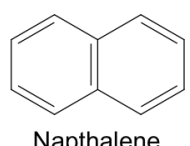

Napthalene

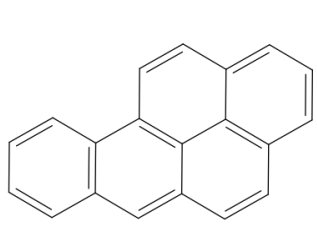

Benzo[a]pyrene

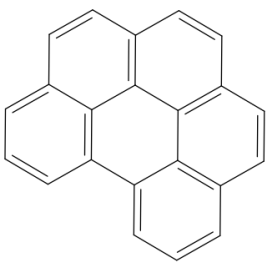

Benzo[g,h,j]perylene

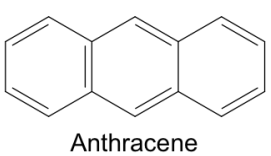

Anthracene

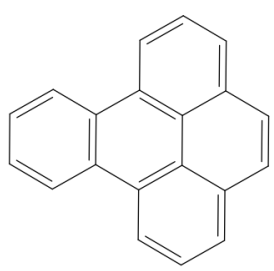

Benzo[e]pyrene

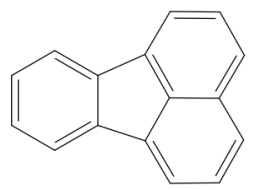

Fluoranthene

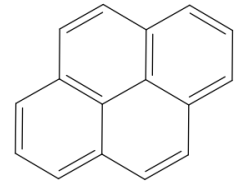

Pyrene

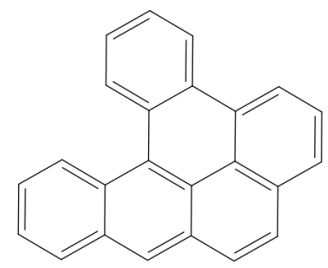

Dibenzo[def,p]chrysene

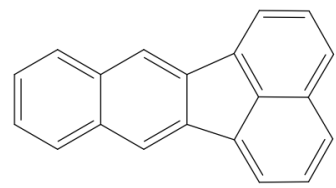

Benzo[k]fluoranthene

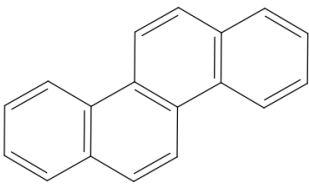

Chrysene

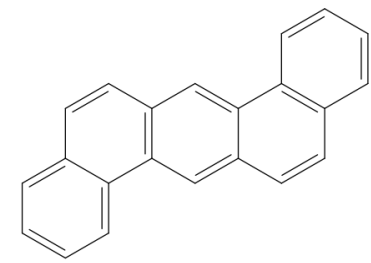

Dibenz $[a, h]$ anthracene

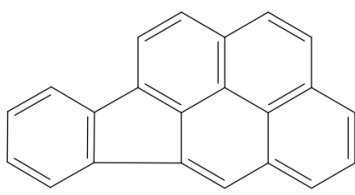

Indeno[1,2,3-cd]pyrene

FIG. 2. Chemical structures for example polycyclic aromatic hydrocarbons. 
PAHs present in the environment usually originate from anthropogenic sources and are formed during incomplete combustion of organic matter. Shen et al. (2013) reported the major global atmospheric emissions of PAHs in 2007 to be residential/commercial biomass burning (60.5\%), open-field biomass burning (agricultural waste burning, deforestation, and wildfire, $13.6 \%)$, and motor vehicles (12.8\%). Natural events such as forest fires, natural oil seeps and volcanic activity also release PAHs into the atmosphere. Thermal decomposition of nearly any organic material may lead to the formation of PAHs. Other important anthropogenic sources of PAHs include cigarette smoke, industrial coke production, asphalt roads, coal tar, incineration, industrial power generation and hazardous waste sites (Luch, 2005). Benzo[a]pyrene $(\mathrm{BaP})$ is one of the most common PAHs detected in the environment; annual production of BaP was estimated to range between 300 and 1,300 metric tons. An estimated 11,000 metric tons of all PAHs were released to the atmosphere on an annual basis (ATSDR, 1995).

PAHs are widely distributed in human food as a result of contamination from air, soil and water, or as a byproduct of certain food preparation processes (e.g., frying, grilling, smoking or charbroiling fish or meat) (Phillips, 1999). Amounts of PAHs present in food are highly variable, but can be as high as parts per billion, depending on the food item and the way that it is prepared. For non-smokers, food is considered one of the main sources of human PAH exposure in the general public. The average total daily intake of PAHs by a member of the general population has been estimated to be $0.207 \mu \mathrm{g} / \mathrm{day}$ from air, $0.027 \mu \mathrm{g}$ /day from water, and 0.16 to $1.6 \mu \mathrm{g} /$ day from food (ATSDR, 1995). Tobacco smoke is one of the most important sources of PAH exposure indoors. Cigarettes contain 
72 known human or animal carcinogens, including BaP at 13.5 ng per cigarette and the most abundant carcinogen, $N^{\prime}$-nitrosonornicotine (NNN) at $70.5 \mathrm{ng}$ per cigarette (Hecht, 2011).

More than two centuries ago, Percivall Pott (1775), a surgeon in St. Bartholomew's Hospital in London, England, described the occurrence of scrotal cancer in chimney sweeps and traced it to the contamination of the skin by soot, now known to be a major source of PAHs. Nearly 100 years later, Volkmann and Bell described several cases of scrotal skin tumors among workers in the German and Scottish paraffin industry (Bell, 1876; Volkmann, 1874). The first official acknowledgment that cancer of any cutaneous site could be caused by pitch, tar or tarry substances was made in an addendum to the Workmen's Compensation Act of Great Britain in 1907, which stated that "scrotal epithelioma occurring in chimney sweeps and epitheliomattous cancer or ulceration of the skin occurring in the handling or use of pitch, tar or tarry compound" (Henry, 1947).

Following these observations, researchers embarked on a series of systematic studies to address the potential for PAHs to cause cancer. Yamagiwa and Ichikawa (1918) were successful in achieving the production of malignant epithelial tumors by repetitive application of coal tar to the ear skin of rabbits. Further evidence that PAHs induce skin tumors in animals was obtained using ethereal extracts of soot (Passey and Carter-Braine, 1925). Around 1930, several researchers worked on the isolation and synthesis of hydrocarbons from coal tar (Cook et al., 1933; Hieger, 1930; Kennaway, 1930; Kennaway and Hieger, 1930). During this time, BaP was first isolated and identified from coal tar (Cook, et al., 1933). Boyland and Levi (1935) discovered urinary 
metabolites of small PAHs, such as anthracene and suggested that toxic hydrocarbons might either be converted into more active metabolites or be detoxified by conversion into other compounds.

The two PAHs, dibenzo[def,p]chrysene (DBC) and BaP have been classified by the International Agency for Research on Cancer (IARC) as group 2A (probably carcinogenic to humans) and group 1 (carcinogenic to humans), respectively. The IARC reported that $\mathrm{BaP}$ produced tumors in all species tested (mouse, rat, hamster, guinea-pig, rabbit, duck, newt, monkey) for which data were reported following exposure by many different routes (oral, dermal, inhalation, intratracheal, intrabronchial, subcutaneous, intraperitoneal, intravenous) yielding many different tumor types (cancer of the liver, stomach, tongue, esophagus, lung, skin, breast, lymphoid and abdomen) (reviewed in (International Agency for Research on Cancer, 2012). DBC has been reported by the IARC to produce tumors in mice, rats, hamsters and fish with cancers of the skin, ovary, testicle, liver, stomach, lung, lymphoid and breast (reviewed in International Agency for Research on Cancer, 2010). With DBC as the most mutagenic and carcinogenic of all the PAHs (Bostrom et al., 2002) and BaP as the most extensively studied, these two PAHs are representative prototypes for environmental PAH toxins.

Women are unavoidably exposed to environmental pollutants during pregnancy. In non-occupational settings, most PAH exposures for non-smoking persons are associated with diet. The developing fetus and neonate are especially sensitive to chemical insults due to increased rates of cell division and underdeveloped detoxification and elimination pathways (Burdge and Lillycrop, 2010; Perera et al., 1999). Cancer risk 
in adult life may be affected by transplacental exposure to environmental chemicals, resulting from greater relative DNA damage and a more rapid frequency of mutations during fetal development (Whyatt et al., 2001). Evidence suggests that many carcinogens are bioactive when administered transplacentally or perinatally and could be linked with childhood and adult cancers (Anderson, 2004; Anderson et al., 2000; Flower et al., 2004; Jensen et al., 2004; Lightfoot and Roman, 2004; Ma et al., 2002; Perera et al., 2005; Perera et al., 2004; Sandler et al., 1985; Sasco and Vainio, 1999; Tang et al., 2006; Whyatt et al., 2000; Zahm and Ward, 1998). Exposure during gestation and throughout breast feeding represents a significant portion of lifetime exposure to PAHs (Somogyi and Beck, 1993).

Studies using animal models have provided further evidence that transplacental exposure to environmental pollutants including PAHs, cigarette smoke, arsenic and benzene can induce carcinogenesis in offspring (Badham et al., 2010; Nicolov and Chernozemsky, 1979; Waalkes et al., 2007; Yu et al., 2006a). Scientists at Oregon State University demonstrated that $\mathrm{DBC}$ is a transplacental carcinogen in the mouse offspring from a cross of strains B6129F1/J dams and 129S1/SvlmJ sires (Yu, et al., 2006a). Mice treated with DBC had a high incidence of mortality due to aggressive T-cell lymphoblastic lymphoma as early as three months of age. Furthermore, mice surviving to ten months of age had a 100\% incidence of lung adenomas (Yu, et al., 2006a). In subsequent studies, this group determined the critical role of CYP1B1 in DBC-initiated transplacental carcinogenesis in the development of the T-cell lymphoblastic lymphoma (Castro et al., 2008a). A cross fostering study demonstrated that the two to three day in 
utero exposure to DBC resulted in much higher lymphoma-associated mortality than exposure to the residual DBC in the breast milk over three weeks of nursing, thus establishing that a brief exposure in utero is primarily responsible for the cancer response (Castro et al., 2008b). All the previously mentioned transplacental mouse models utilized a single dose of $15 \mathrm{mg} / \mathrm{kg}$ administered on gestation day (GD) 17. To more closely model episodic exposure throughout pregnancy, researchers dosed pregnant dams with four smaller amounts of DBC (3.75 mg/kg) on GD 5, 9, 13, and 17 (Shorey et al., 2012). This multiple dosing regimen lessened the lung tumor response in offspring at 10 months of age and eliminated mortality due to T-cell lymphoblastic lymphoma. These observations suggest that the target tissue response for transplacental PAH carcinogenesis in this mouse model is largely dependent upon the stage of fetal development upon exposure.

Many chemical carcinogens exist in the environment as inert procarcinogens that require biological activation. Such is the case for PAHs, which are procarcinogens requiring metabolic activation by phase I cytochrome (CYP) P450 enzymes to generate their highly carcinogenic metabolites. CYP1A1 and CYP1B1 of the CYP1 family are the main enzymes to carry out metabolic activation of PAHs, while the other CYP enzymes in humans that play a lesser role in PAH metabolism, including CYP1A2, CYP2C9, CYP3A4 and CYP3A5 (Shimada and Fujii-Kuriyama, 2004). Metabolic activation of $\mathrm{BaP}$ is initiated by CYP1A1 oxidation to generate (+)- and (-)-BaP-7,8-oxides (Fig. 3). Then, epoxide hydrolase hydrolyses these oxides to produce (-)- and (+)-BaP-7,8-diol, which are further activated by CYP to highly reactive bay region epoxides, $(+)$-BaP-7,8diol-9,10-epoxide. The unstable epoxide intermediates may undergo conjugation with 

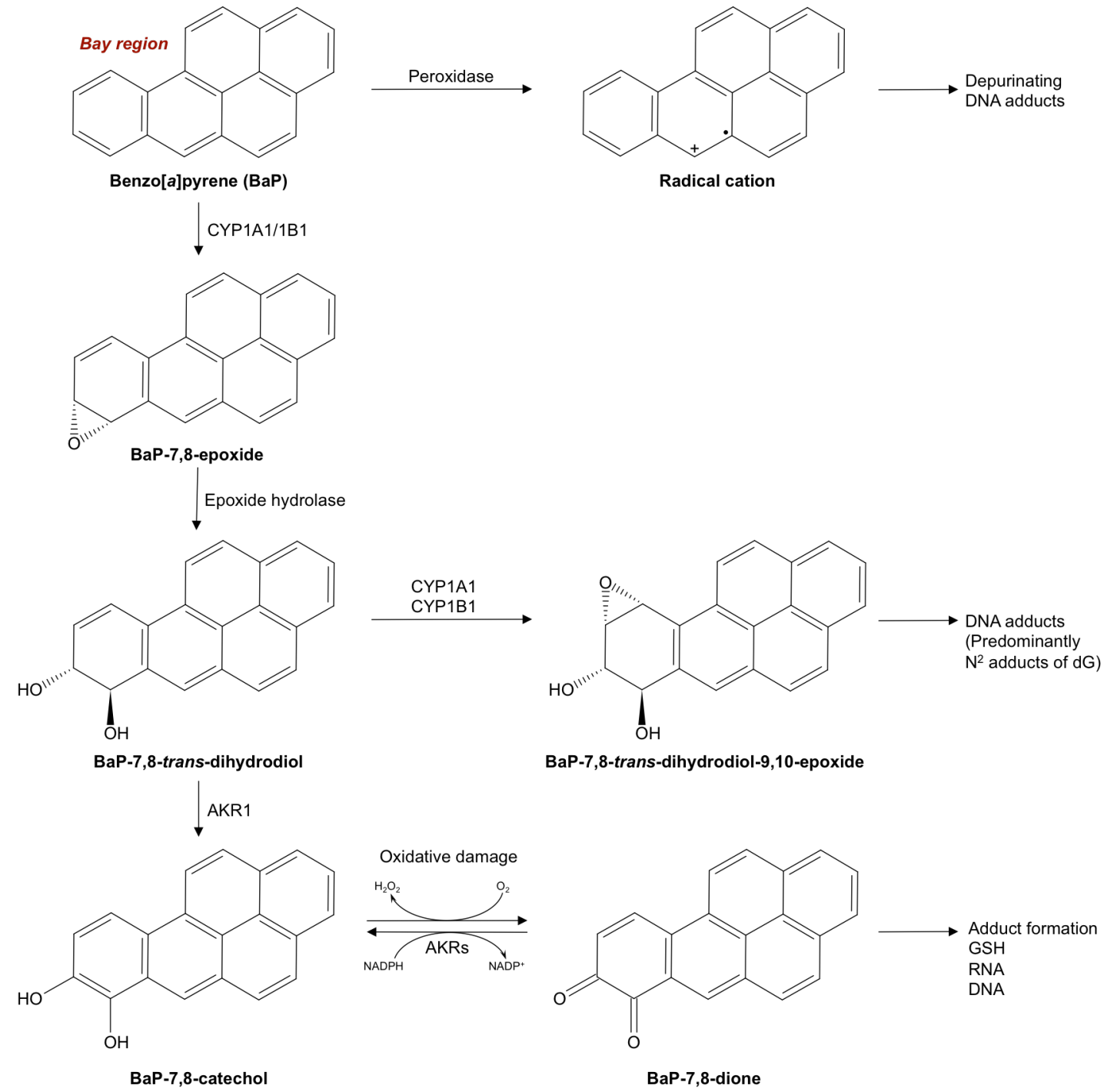

FIG. 3. Pathways for metabolic activation of benzo[a]pyrene.

glutathione (GSH) catalyzed by glutathione-s-transferases (GST) (Armsrong, 1997), nonenzymatic isomerization to phenols, or hydration catalyzed by epoxide hydrolase to form trans-dihydrodiols (Guenthner and Oesch, 1981). The GSH-conjugated metabolites are primarily excreted in the bile or transported to the kidney where they are converted to urinary excretable mercapturic acid conjugates (Hall and Grover, 1990). The phenol 
conjugates may undergo further conjugation reactions with glucuronic acid or sulfate catalyzed by UDP-glucoronosyltransferase (UGT) and sulfotransferases (SULT), respectively. Both the sulfate and glucuronide conjugates are water soluble and easily excreted from the body (Burchell et al., 1997; Duffel, 1997). The highly reactive diolepoxide metabolites may undergo enzymatic conjugation with GSH or, alternatively, may covalently bind to cellular nucleophiles such as DNA, thereby forming DNA adducts (Hall and Grover, 1990). The stereo selectivity of PAH metabolism determines the configuration of the formed DNA adducts in each individual case. Depending upon the configuration of certain groups around the chiral center, there will theoretically be multiple conformations for any given DNA adduct (Harvey, 1991). BaP predominantly reacts with deoxyguanosine $(\mathrm{dG})$ bases while $\mathrm{DBC}$ predominantly binds to deoxyadenosine (dA) residues to form DNA adducts (Mahadevan et al., 2007). However, not all DNA adduct formations have the same biological effect. The BaP-7, 8-diol-9, 10epoxide is a classical example which may form 16 different conformations in double stranded DNA. The (+)-trans-anti-BaPDE- $\mathrm{N}^{2}-\mathrm{dG}$ renders a high tumorigenicity (Conney, 1982; Huberman et al., 1976; Newbold and Brookes, 1976; Phillips, 1983; Weinstein et al., 1976).

Fjord region PAHs, such as DBC, are the most carcinogenic PAHs tested, thus far (Bostrom, et al., 2002; Higginbotham et al., 1993). Their potency is due, in part, to repulsive interactions between the two opposing hydrogen bonds present at the fjord region (Fig. 4). This repulsion renders the molecule out of plane such that the dihyrodiolepoxides can bind more extensively to DNA as compared to PAHs with only a bay region 


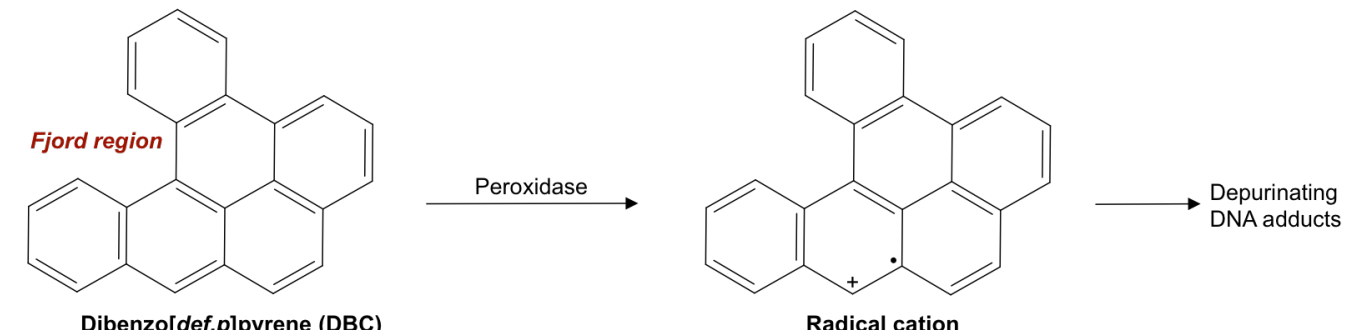

Dibenzo[def,p]pyrene (DBC)

Radical cation

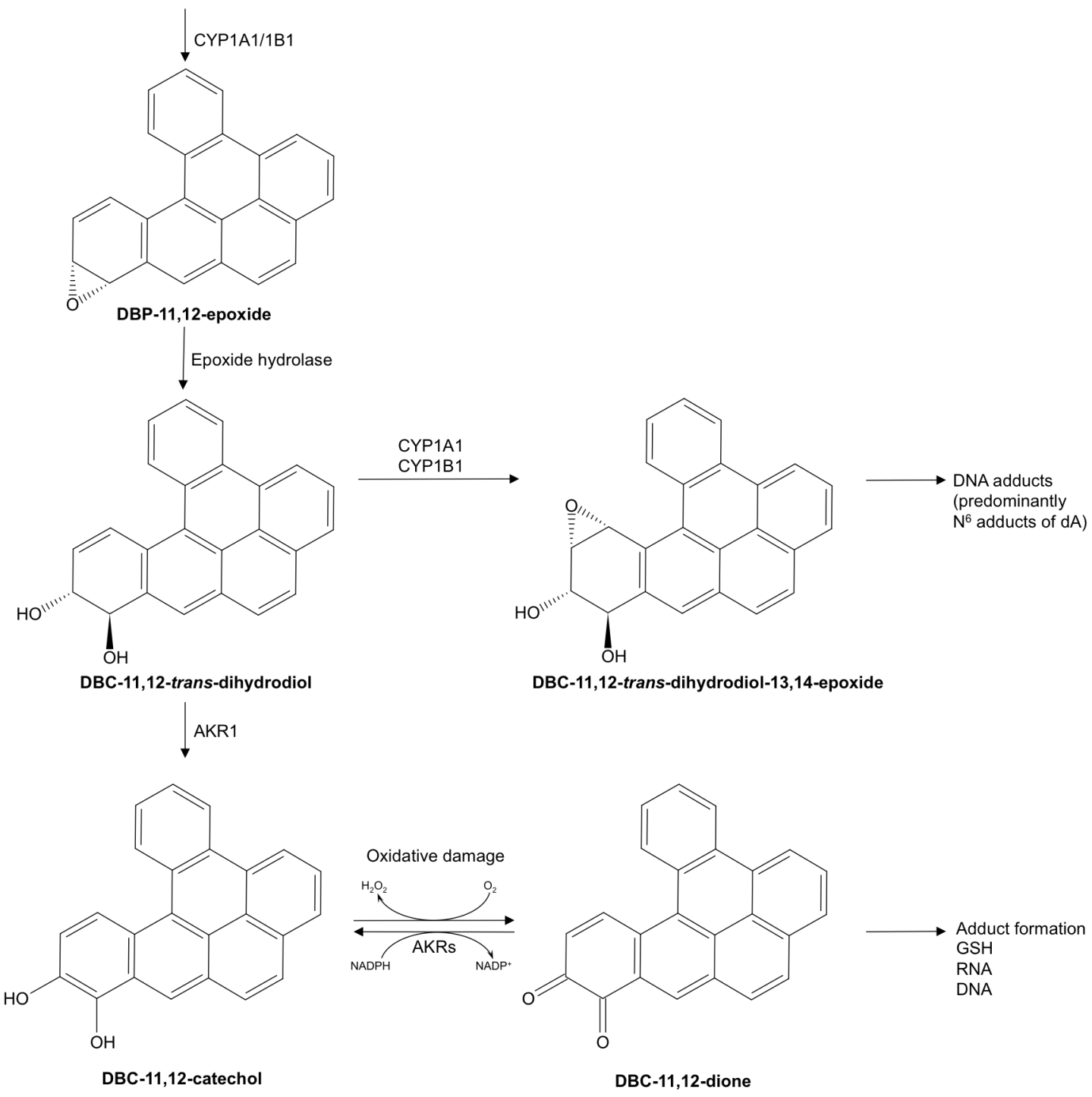

FIG. 4. Pathways for metabolic activation of dibenzo[def,p]chrysene. 
(e.g., $\mathrm{BaP})$ (Geacintov et al., 2002; Wu et al., 2002). These structural effects of the fjord region conformation provide resistance to removal by DNA repair enzymes, leading to a higher incidence of tumor initiation (Buterin et al., 2000; Chakravarti et al., 2000). Expression of CYP1A1, CYP1A2 and CYP1B1 is induced by the presence of PAHs (Iwanari et al., 2002). Exposure to PAH procarcinogens increases the expression of the phase I enzyme that is responsible for their bioactivation. The induction of CYP1A1 expression is regulated by the intracellular aryl hydrocarbon receptor (AhR) to which PAHs chemically bind with high affinity. In the cytoplasm, AhR forms a complex with the $90 \mathrm{kDA}$ heat shock protein, the $\mathrm{X}$-associated protein 2 and co-chaperone protein. Following ligand binding, the AhR complex translocates into the nucleus, dissociates from the protein complex and binds to a nuclear protein called the AhR nuclear translocator (ARNT). Formation of the AhR-ARNT heterodimer converts the complex into its high affinity DNA binding form, which subsequently binds to its specific DNA recognition sites, the xenobiotic responsive elements (XREs), located upstream of the CYP1A1 gene. The binding of XRE results in an increase in chromatin and nucleosome disruption, an increase in promoter accessibility and an increase in the transcription of the CYP1A1 gene (Denison et al., 2002; Whitlock, 1999).

Expression of phase I enzymes appears to play a role in the site of cancer development as demonstrated by their tissue specific expression patterns (Choudhary et al., 2003; Choudhary et al., 2005; McKinnon et al., 1991; Saarikoski et al., 1998). CYP1A1 is primarily expressed in extrahepatic tissues such as the lung (Nelson et al., 1996). The CYP1B1 gene is highly expressed in many human organs including brain, 
endometrium, placenta, fetal adrenal glands, lung, kidney, and lymphocytes (Hakkola et al., 1997; Sutter et al., 1994), although its expression in the liver is rather low. CYP1B1 has been shown to activate $\mathrm{BaP}$ to its ultimate carcinogen metabolite at rates higher than CYP1A2 but lower than CYP1A1 (Kim et al., 1998). On the other hand, CYP1A1 and CYP1B1 appear to differ in their stereo-selective activation of DBC. CYP1B1 has been shown to form higher portions of the highly carcinogenic metabolite of DBC, as compared to CYP1A1 (Luch et al., 1998).

\section{Epigenetics and Lung Cancer}

The term epigenetics refers to specific patterns of chromatin structure and DNA modifications that remain constant through the rounds of cell division, but that do not involve changes to the DNA sequence. The epigenome is another layer of cellular information that controls, registers or signals altered chromosome and gene activity (Bird, 2007). Genes can become inactivated or permanently silenced by at least three pathways: 1) a gene can become mutated and lose its functionality, 2) a gene can become completely lost and thus not be available to perform its function and 3) a gene that has not been lost or mutated can be switched off through changes to its epigenetic code. Different combinations of pathways participate and cooperate with each other to cause either a gain of function or loss of function in critical regulatory genes (Baylin and Jones, 2007). Genetic and epigenetic abnormalities can cause heritable disruptions to homeostatic pathways by two different mechanisms. Either the activation of an oncogene can occur resulting in a gain of function or tumor suppressor genes can be inactivated resulting in a loss of function (Hanahan and Weinberg, 2000; Herman and Baylin, 2003; 
Jones and Baylin, 2002). These epigenetic changes can involve histone modifications, chromatin remodeling and methylation of cytosine in $\mathrm{CpG}$ sites within the promoter regions of genes.

Histone modifications and chromatin remodeling are two interrelated processes that are regulated by post translational modifications of the nucleosome. The base unit of packaged DNA is called a nucleosome, which consists of 146 base pairs (bp) of DNA wrapped around an octamer of four core histone proteins (Luger et al., 1997). Histone proteins are an essential part of DNA packaging and chromosome structure. The Nterminus amino acid tail of the histone protein protrudes away from the nucleosome which provides multiple sites for post translational modifications, including acetylation, methylation, ubiquitination and phosphorylation, that establish the histone code (reviewed in Delage and Dashwood, 2008). These epigenetic modifications regulate gene expression through the remodeling of the chromatin state. For example, methylation of particular residues on histone tails is key modification for regulating chromatin packaging and gene expression. In addition, acetylation of specific sites of the histone protein aid in the regulation of chromatin folding and transcriptional activation. Transcriptionally active regions of the genome reside in euchromatin, a decondensed chromatin state, whereas inactive regions reside in heterochromatin defined as highly compacted chromatin state. Due to the variety of epigenetic modifications, chromatin is a highly dynamic structure, prone to remodeling and restructuring as it receives input from upstream signaling pathways. The histone code works in concert with the DNA 
methylation code to ensure an efficient regulation of chromatin structure and genes transcription (Delage and Dashwood, 2008).

CpG methylation can contribute to tumorigenesis via one of at least three mechanisms. These include global hypomethylation of the genome, focal hypermethylation of the proximal promoter region of tumor suppressor genes and transversions, in which meCpG is converted to TpG (Baylin and Jones, 2007). Three different types of DNA methyltransferases (DNMTs) are responsible for the methylation of DNA. DNMT1 is responsible for the maintenance methylation or the upkeep of the methylation code during cell replication. DNMT3a and $3 \mathrm{~b}$ are responsible for the de novo methylation, that is, the initial pattern of methylation established during early development.

The misregulation of genes controlling cell proliferation, cell cycle, DNA repair, inflammation, growth suppressor evasion, angiogenesis and cell metabolism can contribute to the development of tumorigenesis (Hanahan and Weinberg, 2011). Lung cancer involves an accumulation of genetic and epigenetic events in the cell (Dumitrescu, 2012). Alterations of the epigenome have been associated with many cancers, in which a general pattern of DNA global hypomethylation and promoter hypermethylation have been observed. Although mutations in tumor suppressor genes and oncogenes are important for tumorigenesis, epigenetic modifications that also arise in response to DNA damage have a crucial role in cellular selection, leading to a growth advantage for the tumor cells at the expense of the host (Timp and Feinberg, 2013). Epigenetic alterations are, in fact, more frequent than somatic mutations in lung cancer (Brzezianska et al., 
2013). Epigenetic variation in progenitor cells together with genetic lesions drives tumor progression. This early role for epigenetic alterations in cancer is in addition to epigenetic alterations that can substitute for genetic variation later in tumor progression. Epigenetically disrupted tumor suppressor genes in progenitor cells might be crucial targets for cancer risk assessment (Feinberg et al., 2006). Promoter methylation of specific tumor suppressor genes, along with the overall number of hypermethylated genes, increased with neoplastic progression from hyperplasia to adenocarcinoma (Chung et al., 2011; Licchesi et al., 2008).

There have been numerous studies examining the functions of gene inactivation by promoter hypermethylation with respect to impacting tumorigenesis. Cyclindependent kinase inhibitor $2 \mathrm{a}\left(C d k n 2 a\right.$, also known as $\left.p 16^{I N K 4 a}\right)$ was the first tumor suppressor gene found inactivated in lung cancer predominantly through aberrant gene promoter hypermethylation (Merlo, et al., 1995). Cdkn2a inhibits the cyclin-dependent kinases 4 and 6, which bind cyclin D1 and phosphorylate the retinoblastoma tumor suppressor gene (Lukas et al., 1995; Weinberg, 1995). Since the identification of Cdkn2a inactivation by promoter hypermethylation, epigenetic silencing has been associated with numerous other genes in lung cancer (Belinsky, 2004; Tsou et al., 2002). Methylation of the death-associated protein kinase (Dapkl) and retinoic acid receptor $\beta$ (Rarb) genes were detected in approximately half of alveolar hyperplasia induced mouse lungs chronically exposed to 4-(methylnitrosamino)-1-(3-pyridyl)-1-butanone (NNK) (Pulling, et al., 2004; Vuillemenot, et al., 2004). Loss of function for these two genes could significantly affect cellular apoptosis and normal cell differentiation control. $\mathrm{O}^{6}$ - 
methylguanine-DNA methyltransferase $(M g m t)$ is a DNA repair enzyme that protects cells from the carcinogenic effects of alkylating agents by removing alkyl adducts from the $\mathrm{O}^{6}$ position of guanine. The predominant mechanism for $\mathrm{Mgmt}$ gene inactivation is hypermethylation within its promoter region that leads to transcriptional silencing in $27-$ $47 \%$ of lung adenocarcinomas (Esteller, et al., 1999; Furonaka, et al., 2005). The important functions of these genes and others inactivated through promoter hypermethylation supports a role for their involvement in the initiation and progression of lung carcinogenesis.

Several in vitro studies have shown that treatment of human cells with $\mathrm{BaP}$ or the carcinogenic metabolite benzo[a]pyrene-diolepoxide (BPDE) results in altered histone modification (Sadikovic et al., 2008), gene-specific DNA hypo-and hypermethylation (Damiani et al., 2008; Sadikovic and Rodenhiser, 2006) and altered expression of key genes involved in cancer cell transformation (Damiani, et al., 2008). Damiani, et al. (2008) functionally linked BPDE-induced changes in DNA methylation in transformed human bronchial epithelial cells from smokers with lung cancer to increased expression of DNMT1 (but not DNMTs 3a or 3b) and de novo methylation of a number of tumor suppressor genes, including E-cadherin, H-cadherin, protocadherin-10, Rassf2a and Pax $5 \alpha$ among others. These early studies provide initial evidence that PAHs, specifically $\mathrm{BaP}$, are capable of epigenome modification. However, it is not known whether in vivo exposure to PAHs disrupts patterns of gene promoter methylation. Moreover, it is also unknown whether transplacental exposure to PAHs alters the fetal/neonatal epigenome, specifically. 
Table 1 shows the methylation prevalence of four specific tumor suppressor genes known to be hypermethylated in human lung cancer and some mouse chemical cancer models. These four genes have diverse functions in carcinogenesis and were the focus of a major portion of the present study. Methylation prevalence is the ratio of the number of occurrences for detected methylation in the population studied.

\section{Project Objectives and Hypothesis}

The objective of this project was to determine the impact of transplacental exposure of PAHs on the neonatal and adult offspring epigenome in the mouse lung. We hypothesized that transplacental exposure to PAHs alters DNA methylation in the promoter region of key tumor suppressor genes leading to increased risk of lung cancer in the adult. The specific objectives were as follows:

1. Determine the impact of transplacental exposure to $\mathrm{DBC}$ and $\mathrm{BaP}$ on methylation of the promoter regions of the Cdkn2a, Rarb, Dapkl and Mgmt genes in neonate mouse lung. Determine the impact of transplacental exposure to $\mathrm{DBC}$ and $\mathrm{BaP}$ on genome-wide methylation of the lung tumors in the adult offspring mice.

2. Assess the timing of tumor suppressor gene silencing resulting from gestational PAH exposure by measuring gene promoter methylation in adult mice (aged 15 to 45 weeks) as preneoplastic lesions develop into lung adenocarcinomas in the adults. 


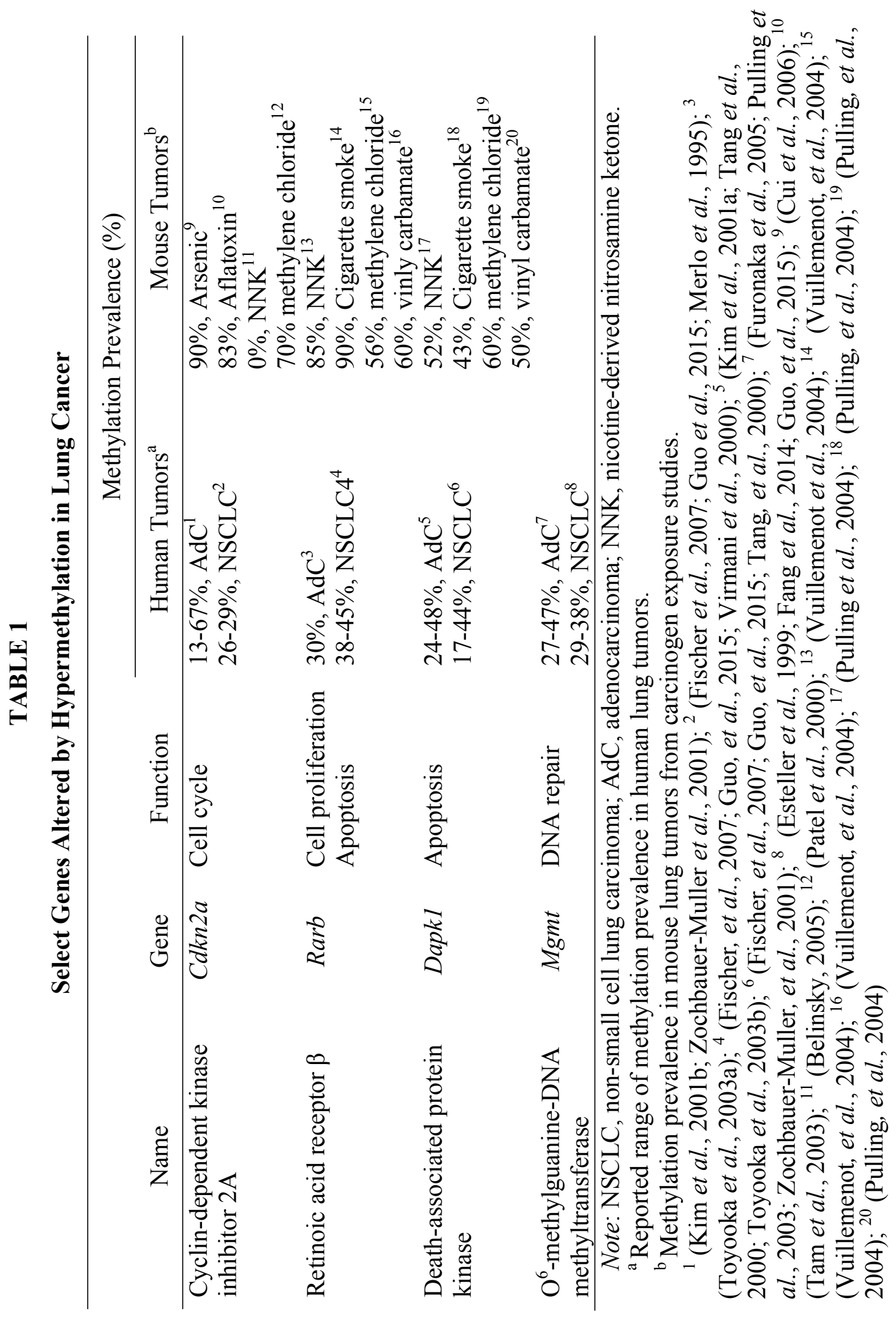




\section{MATERIALS AND METHODS}

\section{Chemicals}

DBC and BaP were purchased from Sigma-Aldrich (St. Louis, MO; CAS No. 191-30-0, CAS No. 50-32-8). Buffered 10\% neutral formalin was purchased from VWR (Houston, TX). Optimal Cutting Temperature (OCT) Compound was obtained from Sakura Finetek (Torrence, CA). Arcturus ${ }^{\circledR}$ HistoGene ${ }^{\circledR}$ staining solution was purchased from Applied Biosystems (Foster City, CA). All other chemicals used were of reagent grade and purchased from general laboratory suppliers.

\section{Animals}

The Utah State University Institutional Animal Care and Use Committee approved all procedures for the handling and treatment of mice used in this study (Protocol \#1442). Animals were housed in the USTAR BioInnovations Center's Vivarium at Utah State University. All mice were maintained on a 12:12 hour dark:light cycle, and water was provided ad libitum. Eight week old B6129SF 1/J females and 129S1/SvImJ males were obtained from the Jackson Laboratory (Bar Harbor, ME) and bred to obtain a backcross strain that is sensitive to $\mathrm{DBC}$ and $\mathrm{BaP}$ as transplacental carcinogens (Castro, et al., 2008a; Yu, et al., 2006a). Moreover, B6129SF1/J mice possess an AhR-responsive allele, meaning that these mice are prone to carcinogenesis induced by PAHs via induction of Cyp1b1 and/or Cyp1a1 (Yu, et al., 2006a). Furthermore, the mouse Cyp1b1 has the highest activity toward conversion of DBC to the carcinogenic fjord region diol-epoxide (Castro et al., 2008c; Luch, et al., 1998). Dams 
were fed AIN93G (Harlan-Teklad, Madison, WI) during pregnancy and lactation. Weaned offspring were fed AIN93G until three months of age, and were then fed AIN93M diet (Harlan-Teklad, Madison, WI) ad libitum until euthanasia.

\section{Experiment Design}

B6129SF1/J dams and 129S1/SvImJ sires were bred and day one of gestation was determined by the detection of the vaginal plug. On gestation days 5, 9, 13 and 17, pregnant mice were dosed by body weight with vehicle ( $5 \mathrm{ml} / \mathrm{kg} /$ day corn oil), 3.75 $\mathrm{mg} / \mathrm{kg} /$ day DBC (total dose $15 \mathrm{mg} / \mathrm{kg}$ ) or $12.5 \mathrm{mg} / \mathrm{kg} /$ day BaP (total dose $50 \mathrm{mg} / \mathrm{kg}$ ). Any mice exhibiting signs of morbidity, pain or distress during the study were humanely euthanized with an overdose of $\mathrm{CO}_{2}$ and necropsied.

For the sham treatment group (group 1), 11 females were bred to 5 males to generate 93 pups. For the DBC group (group 2), 13 dams were bred to 7 males to generate 89 pups. Because $\mathrm{BaP}$ is a less potent carcinogen compared to $\mathrm{DBC}$, we generated more animals for group 3 using 15 dams and 8 males to produce 131 offspring. Four litters from each group were used to obtain lung tissue from neonate offspring at day one of age. From these neonates, lung, liver, thymus and heart were collected and immediately frozen in liquid nitrogen and stored at $-80^{\circ} \mathrm{C}$. The tissues from all pups within each litter were combined to provide sufficient tissue for analysis, resulting in $n=$ 4 for each experimental group.

At ages 15, 25, 35 and 45 weeks, six mice from group 1 and 2 and ten mice from group 3 were randomly selected to be euthanized to obtain tissues samples throughout the progression of lung tumor development, including normal tissue, preneoplastic lesions 

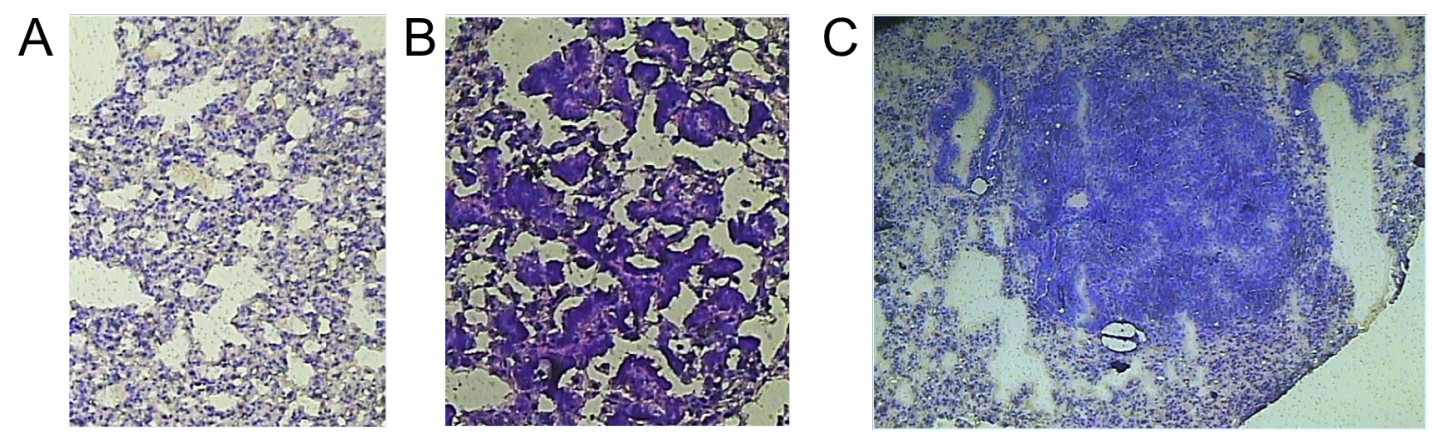

FIG. 5. Representative microscopy images of mouse lung tissues collected for laser capture microdissection. (A) normal lung tissue, (B) atypical adenomatous hyperplasia, and (C) adenocarcinoma.

(atypical adenomatous hyperplasia $[\mathrm{AAH}]$ ) and lung adenocarcinomas. Example images depicting these tissue types in mouse lung are shown in Fig. 5. Lung tissues were collected and embedded in OCT for later processing and use in laser capture microdissection (LCM). At 45 weeks, all remaining offspring from all experimental groups were humanely euthanized by $\mathrm{CO}_{2}$ asphyxiation and necropsied. Lung tumors present on the lungs of these 45 week-old mice were identified, counted and the diameter was measured by digital caliper. Half the lung was placed in the $10 \%$ neutral buffered formalin, while the other half was frozen in liquid nitrogen and stored at $-80^{\circ} \mathrm{C}$. At each necropsy, body weights and spleen, kidney, liver, and heart weights were recorded; these organs were stored in 10\% buffered neutral formalin.

\section{Lung Tissue Collection and Processing}

Lung tissues designated for analysis by LCM were processed in two halves, each of which was placed into a $12 \times 12 \times 20 \mathrm{~mm}$ embedding mold, covered with OCT compound and then submerged in chilled isopentane until solidification of OCT, 
according to the supplier's protocol. Frozen tissue blocks were stored at $-80^{\circ} \mathrm{C}$ until further processing.

Sectioning of the tissue blocks was completed using the Leica CM1950 cryostat (Leica Biosystems, Buffalo Grove, IL). Tissue sections were cut with $8 \mu \mathrm{m}$ thickness and placed onto Arcturus ${ }^{\circledR}$ polyethylene naphthalate (PEN) membrane slides (Applied Biosystems, Foster City, CA). The slides were immediately placed into a microslide box on dry ice. Next, slides were immersed in $75 \%$ ethanol (v/v) for $30 \mathrm{sec}$ followed by distilled water for $30 \mathrm{sec}$ and then removed. Next, $100 \mu \mathrm{l}$ of Arcturus ${ }^{\circledR}$ HistoGene ${ }^{\circledR}$ staining solution was applied to each slide for $20 \mathrm{sec}$. Then, slides were sequentially immersed for $30 \mathrm{sec}$ each in distilled water, 75\% (v/v) ethanol, 95\% (v/v) ethanol and $100 \%$ ethanol and then finally $5 \mathrm{~min}$ in xylene. The slides were dried in a ventilated hood for an additional five minutes and then stored at $-80^{\circ} \mathrm{C}$ until LCM.

Slide sections were examined by a board certified Veterinary Pathologist at the Utah Veterinary Diagnostics Laboratory to localize tissue types to be collected by LCM. For this analysis, only tissues from mice in group 1 (sham) and group 2 (DBC-initiated) were used. Tissue types collected for LCM included normal adjacent lung, AAH and lung adenocarcinomas for mice aged 25 to 45 weeks initiated with DBC in utero (group 2). Only normal lung tissue was collected for mice in group 1 at all ages and for mice in group 2 at age 15 weeks as there was no evidence of AAH in DBC-initiated offspring at this age. Tissues were collected from four of the six mice euthanized at each time point, as not all tissue types were evident in every mouse (i.e., AAH was evident in only four of the six mice collected at 25 weeks). 
LCM collection was performed on Arcturus ${ }^{\circledR}$ Veritas ${ }^{\mathrm{TM}}$ laser capture micro dissection instrument at the University of Utah Cell Imaging core facility. LCM was performed using an infrared (IR) laser, which captured the cells of interest directly onto Arcturus ${ }^{\circledR}$ CapSure ${ }^{\circledR}$ Macro LCM caps (Applied Biosystems, Foster City, CA). Caps with tissues were then snapped onto $0.5 \mathrm{ml}$ microcentrifuge tubes, and then DNA from the captured tissues was extracted using the Arcturus ${ }^{\circledR}$ PicoPure ${ }^{\circledR}$ DNA Extraction Kit (Applied Biosystems, Foster City, CA) according to the supplied protocol with no deviation.

Frozen lung tissues from neonate offspring were pooled by litter $(n=4$ litters per group), and DNA and total RNA were isolated from these tissues using TRI Reagent ${ }^{\circledR}$ (Sigma-Aldrich, St. Louis, MO) according to the supplier's protocol.

\section{Roche 454 Sequencing}

DNA collected from LCM-captured lung tissues from group one and two mice and from neonate lungs was utilized for bisulfite deep sequencing. All DNA samples were bisulfite-converted using the EZ DNA Methylation Kit ${ }^{\mathrm{TM}}$ (Zymo Research, Irvine, CA). Then, the bisulfite-converted DNA was subject to a genomic pre-amplification step using Fluidigm's Specific Target Amplification (Preamplification) protocol (Fluidigm, South San Francisco, CA). Table 2 shows the primer sequences utilized for preamplification and library preparation, and Fig. 6 shows the location of primer sets with respect to predicted $\mathrm{CpG}$ islands for each target gene. Genomic sequence information was obtained from the Ensembl Genome Browser (Flicek et al., 2012), and primer sequences were designed using MethPrimer software (Li and Dahiya, 2002). The 
TABLE 2

Primers Used for Bisulfite Sequencing

\begin{tabular}{|c|c|c|c|}
\hline Gene & Primer $\left(5^{\prime}\right.$ to $\left.3^{\prime}\right)$ & Position $^{\mathrm{a}}$ & CpG sites \\
\hline \multicolumn{4}{|c|}{$C d k n 2 a$} \\
\hline For & [CS1]-GATGGATTTGGAGTAAGGGAAA & -366 & 41 \\
\hline Rev & [CS2]-CCCAAAAACRCCCAAAAA & -86 & \\
\hline \multicolumn{4}{|l|}{ Dapk1 } \\
\hline For & [CS1]-GTGTGGGGGTTTTTTAGTTTAGATT & 340 & 22 \\
\hline Rev & [CS2]-СТТССТААТАССТАСССААТТССТC & 635 & \\
\hline \multicolumn{4}{|l|}{ Mgmt } \\
\hline For & [CS1]-GTTTTAGGTTTGGAAGAAGAGGTTT & -280 & 20 \\
\hline Rev & [CS2]-CACAAATTTTAAATACCTAAACACCAA & 15 & \\
\hline \multicolumn{4}{|c|}{ 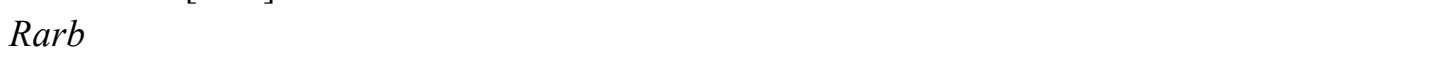 } \\
\hline For & [CS1]-GGTTTGGTTAGGAATAGGAGAGTAGA & -351 & 17 \\
\hline Rev & [CS2]-AACAACCCTACAAAAACCTTCAAC & -165 & \\
\hline
\end{tabular}

Note: For, forward primer; Rev, reverse primer; CS1, common sequence tag 1 (5'acactgacgacatggttctaca-3'); CS2, common sequence tag 2 (5'-tacggtagcagagacttggtct-3'). ${ }^{a}$ Position number specifies the bp location of the genomic primer's 5' end in relation to the first $b p$ of the first exon of the gene.
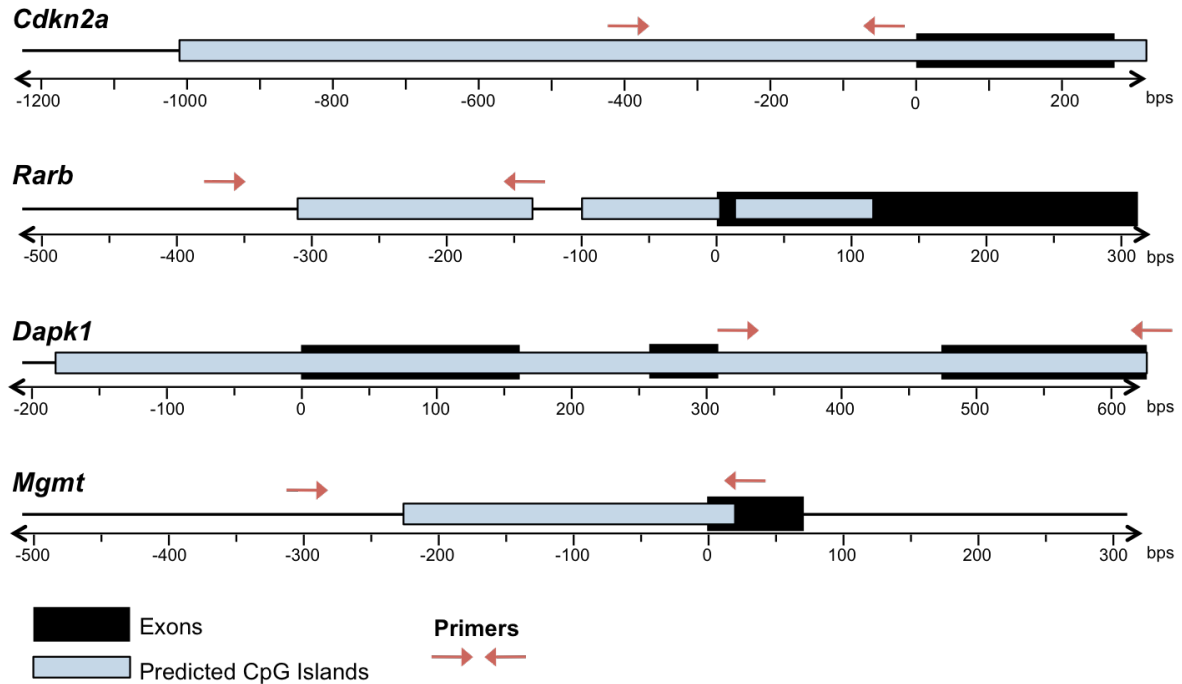

FIG. 6. Location of primer sequences with respect to predicted $\mathrm{CpG}$ islands for selected genes. Red arrow pairs indicate regions targeted for generating amplicons for ultra-deep bisulfite sequencing. The scale indicates position with respect to the transcript start site at exon 1 (first black bar). CpG islands (light blue) were predicted using EMBOSS Cpgplot (Li et al., 2015). 
genome target specific primers were designed to contain a 22 bp Fluidigm forward or reverse common sequence tag (CS1 or CS2) at the 5' end.

The Fluidigm 48.48 Access Array system was used for library preparation of the samples and our four target genes following the manufacturers supplied protocols. Briefly, bisulfite-modified preamplified DNA was combined with master mix from the FastStart High Fidelity PCR System, dNTPack (Roche, Bradford, CT) following manufacturer's protocol and then loaded into the primed 48.48 access array plate. Each plate was run on the BioMark system (Fluidigm, South San Francisco, CA), which utilizes Fluidigm's integrated fluidic circuits for automating the PCR reactions in nanoliter volumes. Three 48.48 access array plates were utilized to amplify all samples, and each gene was duplicated eight times across both plates with one exception. Mgmt was duplicated 16 times on each plate in order to more closely balance the ultimate yield of sequence reads from each amplicon, as amplification of $M g m t$ was roughly half as efficient as the other genes. Products from the Access Array were subjected to electrophoresis on a $2 \%$ agarose gel for visual conformation of adequate amplification and appropriate amplicon size. Gel bands corresponding to each amplicon were excised, and DNA was extracted using Qiagen's QIAquick ${ }^{\circledR}$ Gel Extraction Kit (Qiagen, Valencia, CA). A secondary amplification, using a standard thermocycler, was performed utilizing primers that contained the $25 \mathrm{bp}$ Roche $454 \mathrm{~A}$ and B adaptor sequences (adapter A, CGTATCGCCTCCCTCGCGCCATCAG on the forward primer and adapter B, CTATGCGCCTTGCCAGCCCGCTCAG on the reverse primer), the $10 \mathrm{bp}$ multiplex identifier (MID) and the CS1 or CS2 tags, respectively. This second round of PCR used 
$1 \mu \mathrm{l}$ of the PCR product from the first round and $2 \mu \mathrm{M}$ primer in master mix from the FastStart High Fidelity PCR System, dNTPack (Roche, Bradford, CT) and the following cycling parameters: $95^{\circ} \mathrm{C}$ for $10 \mathrm{~min}$ for $1 \mathrm{cycle}, 95^{\circ} \mathrm{C}$ for $15 \mathrm{sec}$, annealing temperature of $60^{\circ} \mathrm{C}$ for $30 \mathrm{sec}$, and $72^{\circ} \mathrm{C}$ for $1 \mathrm{~min}$ for 15 cycles and the final stage of $72^{\circ} \mathrm{C}$ for $3 \mathrm{~min}$ for 1 cycle. At the conclusion of the second round of PCR, the amplicons contain all the necessary elements for 454 sequencing.

DNA samples were then subjected to electrophoresis on a $2 \%$ agarose gel for visual conformation of adequate amplification and appropriate amplicon size. The DNA bands were excised and DNA was extracted using Qiagen's QIAquick ${ }^{\circledR}$ Gel Extraction Kit. Finally, the fully processed DNA was sent to the Center for Integrated BioSystems at Utah State University to be sequenced on the Roche 454 Sequencer (Roche, Bradford, CT). Briefly, amplicon libraries were subjected to emulsion PCR to produce DNA-coated beads, loaded onto a $70 \times 75$ PicoTiterPlate, and sequenced using the FLX Titanium DNA sequencing Kit according to the manufacturer's protocol.

\section{NimbleGen DNA Methylation Array}

Genome-wide DNA methylation analysis of offspring lung tissues was performed using the NimbleGen Mouse DNA Methylation 3×720K CpG Island Plus RefSeq Promoter Array (Roche NimbleGen Inc., Basel, Switzerland). Tissue types analyzed included normal lung tissue from sham-initiated offspring and normal adjacent lung tissues and lung tumors from 45 week-old offspring initiated with $\mathrm{DBC}$ or $\mathrm{BaP}$ in utero. High-quality genomic DNA was extracted from lung tissue using the DNeasy Blood \& Tissue Kit (Qiagen, Valencia, CA). One microgram of genomic DNA was restriction- 
digested with Mse I restriction enzyme (New England BioLabs, Ipswich, MA) to generate 200 to $1000 \mathrm{bp}$ fragments. Samples were subjected to heat denaturation for $20 \mathrm{~min}$ at $65^{\circ} \mathrm{C}$ in order to stop the reaction. DNA samples were then purified using QIAquick PCR Purification Kit (Qiagen, Valencia, CA), and DNA concentration was determined using a NanoDrop spectrophotometer (Thermo Fisher Scientific). Verification of fragment size, using $200 \mathrm{ng}$ of sample was performed by gel electrophoresis ( $2 \%$ agarose gel). An aliquot of 10 to $15 \mathrm{ng}$ was reserved after quantification for use as control (input DNA). The remainder was immunoprecipitated using the Methylated-DNA IP Kit (Zymo Research, Irvine, CA; Cat. No. D5101). Because the quantity of MeDIP DNA obtained was insufficient for processing the genome-wide arrays, a whole-genome amplification step was required. Whole-genome amplification was performed utilizing a series of two kits with a starting amount of 10 ng for both input and MeDIP DNA as per manufacturer's instructions. The first kit used was GenomePlex ${ }^{\circledR}$ Complete Whole Genome Amplification (WGA) Kit (Sigma-Aldrich Company, St. Louis, MO), or WGA2 kit. The second was GenomePlex ${ }^{\circledR}$ WGA Reamplification Kit (Sigma-Aldrich Company, St. Louis, MO) or WGA3 kit. Following processing of samples through this two-kit procedure, the WGA-amplified DNA was then purified using QIAquick PCR Purification Kit (Qiagen, Valencia, CA), and $5 \mu \mathrm{g}$ of DNA per sample were provided to NimbleGen (Roche NimbleGen Inc, Basel, Switzerland) for array hybridization and preliminary data processing. 
Data Analysis

Statistical analysis of lung tumor multiplicity was performed using one-way ANOVA with Bonferroni's test for multiple comparisons (GraphPad Prism vers. 6, LaJolla, CA). Lung tumor incidence in 45-week old offspring exposed in utero to BaP was analyzed using Fisher's exact test (GraphPad Prism).

Sequencing data generated by Roche 454 were processed using Amplicon Variant Analyzer (Roche, Bradford, CT). Sequences were separated by sample according to their barcode sequences, and barcodes were trimmed off. The resulting sequences were then aligned to reference sequences for each amplicon and the primer sequences were trimmed. BioEdit Sequence Alignment Editor was then used to prepare and edit the individual alignment files for each sample/amplicon (Hall, 1999).

The processed data were subjected to analysis of $\mathrm{CpG}$ methylation frequency using the Bisulfite Sequencing DNA Methylation Analysis (BISMA) program (Rohde et al., 2010). First, data files (limited to 400 sequence reads per file) uploaded to BISMA were automatically subjected to a multiple sequence alignment and quality control analysis on the following parameters: defined quality and specificity criteria of sequence identity, percentage of insertions/deletions, conversion rate and percentage of unresolved annotations. Sequences that did not pass these quality criteria were automatically removed from subsequent analysis. BISMA analysis generated output for each sequence analyzed, including the calculated average methylation at each $\mathrm{CpG}$ position and the average methylation for the entire sequence. Also, the program generated an aligned sequence map depicting methylation status for each $\mathrm{CpG}$ site for each included sequence. 
Data received from NimbleGen's genome-wide DNA methylation analysis consisted of processed data files as well as the raw data files for all samples submitted. Raw data files contained signal intensity data from the scanned images of each array obtained using NimbleScan. Peak data files (processed data files) were generated from the raw signal intensity data by Nimblegen. Briefly, for each array feature, a scaled $\log _{2}$ ratio was calculated as the ratio of the input signals for the experimental and control samples co-hybridized to the array. The $\log _{2}$ ratio was computed and scaled to center the ratio data around zero. Scaling was performed by subtracting the bi-weight mean for the $\log _{2}$ ratio values for all features on the array from each $\log _{2}$ ratio value. From the scaled $\log _{2}$ ratio data, a fixed-length window (750 bp) was placed around each consecutive probe, and the one-sided Kolmogorov-Smirnov (KS) test was applied to determine whether the probes are drawn from a significantly more positive distribution of intensity log-ratios than those in the rest of the array. The resulting score for each probe is the $-\log _{10} \mathrm{p}$-value from the windowed KS test around that probe. Using NimbleScan, peak data files were generated from the p-value data files. NimbleScan detects peaks by searching for at least two probes above a $-\log _{10} \mathrm{p}$-value minimum cutoff of 2 . Peaks within $500 \mathrm{bp}$ of each other were merged. Finally, the "peak score" was calculated as the average $-\log _{10} \mathrm{p}$-values from probes within that peak. Two sets of processed data files with these calculated peak scores were generated by Nimblegen. One set contained data for all peak scores near a given transcript, while the second set included data for only the peak score nearest the transcription start site of a given gene. 
Gene lists for functional ontology analysis were generated by selecting features with peak score $>2$ (indicative of methylated DNA) in all three samples from the treatment group of interest and by excluding all features that were not methylated (peak score $<2)$ in at least 2 of the 3 samples from the comparison treatment group(s). Using these criteria, for example, genes listed as methylated in sham samples were consistently methylated in only the sham tissues and mostly not methylated in other tissues. Gene ontology analysis was performed using the AgriGO singular enrichment analysis (SEA) tool (Du et al., 2010) against the mouse gene ontology database (Mouse Genome Informatics) with the following parameters: Fisher test with FDR under dependency correction and significance level of $P<0.05$ and the minimum number of mapping entries set at five genes. The gene ontology type performed was a generic GO slim (Gene Ontology Consortium). 


\section{RESULTS}

\section{Lung Tumorigenesis in 45 Week-Old Offspring}

Lung tumor incidence was $29.5 \%$ in 45 week-old offspring initiated in utero with $\mathrm{BaP}$, much lower than the $100 \%$ incidence rate observed for the more potent carcinogen DBC (Fig. 7A). The spontaneous lung tumor rate in sham-initiated offspring mice at 45 weeks of age was $8.9 \%$. A similar pattern was observed for lung tumor multiplicity, calculated as the number of lung tumors per tumor-bearing animal (Fig. 7). Shaminitiated mice that developed lung cancer spontaneously did so with a very low multiplicity (one tumor per animal), and multiplicity in BaP-initiated offspring was similarly low with only one to two tumors per animal. Alternatively, DBC-initiated

A

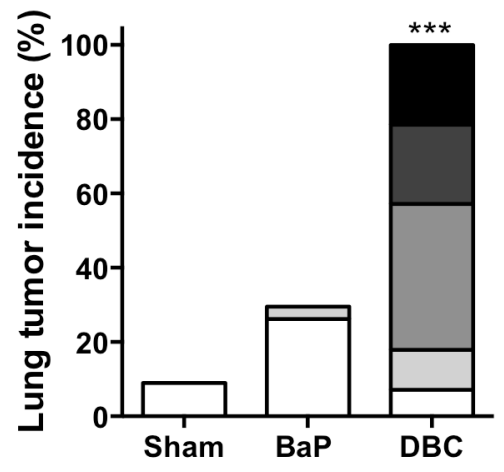

B

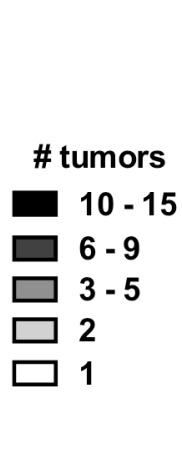

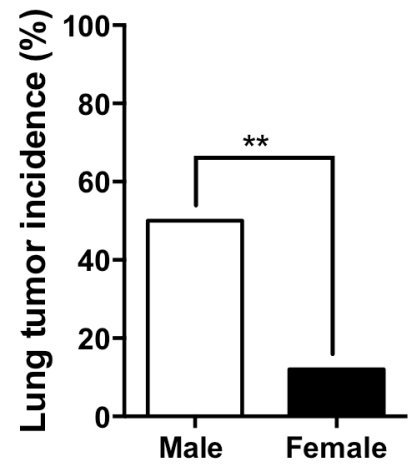

FIG. 7. Lung tumorigenesis in offspring exposed to PAHs in utero. (A) Values for tumor incidence are indicated by the height of the bar for each group, and tumor multiplicity is indicated by bar shading. ${ }^{* *}, P<0.001$ for tumor multiplicity compared to Sham and BaP treatment groups as determined by one-way ANOVA with Bonferroni's post hoc test for multiple comparisons. (B) Values shown are the percent of male and female offspring initiated in utero with $\mathrm{BaP}$ with lung tumors at 45 weeks of age. **, $P=0.0018$ as determined by Fisher's exact test. 
offspring had a significantly higher average tumor multiplicity of six tumors per tumorbearing animal $(P<0.0001)$. Interestingly, lung tumor incidence in 45 week-old offspring exposed in utero to $\mathrm{BaP}$ was significantly lower in females (12\%) compared to their male counterparts $(50 \%)(P=0.0018)$ (Fig. 7B). Although statistical analysis suggested that lung tumor incidence was different between the sexes in mice initiated with $\mathrm{BaP}$, this result should be interpreted with some caution given the relatively small number of mice that developed tumors within this treatment group. There was no apparent difference observed in lung tumor incidence in DBC-initiated mice, as all animals exposed to this carcinogen developed lung cancer, as has been repeatedly observed in this model (Castro, et al., 2008b; Risch, et al., 1993; Yu, et al., 2006a).

\section{Amplicon Bisulfite Sequencing Analysis}

DNA promoter methylation patterns were examined using bisulfite sequencing of CpG sites at single base pair resolution across four genes from DNA collected via LCM. The average percent methylation values corresponding to the $\mathrm{CpG}$ site sequenced are provided in Tables 3-6, with the data organized according to the sample type, animal age and the amplicon sequenced. Unsupervised bi-directional hierarchical clustering was performed using average methylation data for each comparison group to identify distinctions in methylation profiles according to age (neonate, 15, 25, 35 or 45 weeks), type of carcinogen exposure (Sham, DBC or BaP) or tissue type (normal, AAH or tumor). Clustering was also performed using methylation data for each individual mouse to assess variation in methylation profiles within and across age, carcinogen exposure and tissue-type groupings. 
TABLE 3

Average Methylation per CpG Site for the Cdkn2a Promoter Region Sequenced by Roche $454^{\text {a }}$

\begin{tabular}{|c|c|c|c|c|c|c|c|c|c|c|c|c|c|c|c|c|c|c|c|c|c|c|c|c|c|c|c|c|c|c|c|c|c|c|c|c|c|c|c|c|c|c|c|c|}
\hline \multirow[b]{2}{*}{ Carcinogen } & \multirow[b]{2}{*}{ Age } & \multirow[b]{2}{*}{$\begin{array}{l}\text { Tissue } \\
\text { type }\end{array}$} & \multirow[b]{2}{*}{$\begin{array}{l}\text { Number } \\
\text { reads }^{c}\end{array}$} & \multicolumn{41}{|c|}{ CpG Site Number ${ }^{b}$} \\
\hline & & & & 4 & 7 & 14 & 27 & 36 & 43 & 54 & 59 & 66 & 70 & 73 & 82 & 88 & 92 & 98 & 106 & 125 & 129 & 134 & 138 & 140 & 145 & 147 & 150 & 163 & 165 & 175 & 5179 & 184 & +188 & 195 & 200 & 209 & 211 & 221 & 229 & 235 & 245 & 247 & & 9251 \\
\hline Sham & Neonate & Normal & 11 & 0.0 & 0.0 & 0.0 & 0.0 & 0.0 & 4.2 & 0.0 & 0.0 & 0.0 & 0.0 & 0.0 & 0.0 & 0.0 & 0.0 & 0.0 & 0.0 & 0.0 & 0.0 & 0.0 & 0.0 & 0.0 & 0.0 & 0.0 & 0.0 & 0.0 & 0.0 & 16.7 & 722.9 & 22.9 & 912.5 & 0.0 & 0.0 & 0.0 & 0.0 & 0.0 & 0.0 & 0.0 & 0.0 & 0.0 & 0.0 & 0.0 \\
\hline Sham & $15 \mathrm{wk}$ & Normal & 793 & 0.2 & 0.02 & 2.2 & 0.3 & 0.2 & 0.1 & 0.6 & 0.2 & 0.9 & 0.6 & 0.7 & 0.6 & 0.3 & 0.5 & 1.5 & 0.2 & 0.1 & 0.2 & 0.6 & 0.2 & 0.3 & 0.1 & 0.2 & 0.2 & 0.2 & 1.4 & 31.4 & 443.3 & 42.1 & 122.2 & 0.2 & 0.1 & 0.0 & 0.1 & 1.0 & 0.1 & 0.0 & 0.2 & 1.3 & 0.4 & 0.3 \\
\hline Sham & $25 \mathrm{wk}$ & Normal & 1042 & 0.6 & 0.4 & 3.6 & 1.8 & 0.9 & 1.2 & 0.3 & 6.9 & 0.5 & 1.0 & 0.3 & 0.4 & 0.3 & 0.2 & 0.7 & 0.4 & 0.3 & 0.7 & 0.4 & 0.9 & 0.0 & 0.2 & 0.2 & 0.4 & 1.3 & 0.7 & 15.6 & 624.0 & 14.2 & 211.8 & 0.2 & 0.2 & 0.4 & 0.4 & 0.2 & 0.1 & 0.5 & 0.3 & 0.5 & 0.2 & 0.0 \\
\hline Sham & $35 \mathrm{wk}$ & Normal & 915 & 0.2 & 0.2 & 0.2 & 0.8 & 0.6 & 1.0 & 0.1 & 0.6 & 0.5 & 0.3 & 1.0 & 0.0 & 0.2 & 0.7 & 1.7 & 0.6 & 0.2 & 0.8 & 0.5 & 0.5 & 1.2 & 0.2 & 0.2 & 0.3 & 0.7 & 0.8 & 18.4 & 426.6 & 23.2 & 211.4 & 0.5 & 0.8 & 1.1 & 0.5 & 0.2 & 0.2 & 0.7 & 0.1 & 0.3 & 1.3 & 0.2 \\
\hline Sham & $45 \mathrm{wk}$ & Normal & 654 & 0.0 & 0.4 & 0.6 & 1.3 & 0.0 & 0.5 & 0.2 & 0.5 & 0.3 & 0.3 & 0.4 & 0.4 & 0.3 & 0.2 & 0.1 & 0.1 & 0.7 & 0.0 & 0.1 & 3.7 & 0.6 & 0.2 & 0.5 & 0.0 & 0.1 & 0.2 & 55.1 & 160.5 & 61.2 & 230.5 & 1.2 & 0.2 & 0.5 & 0.4 & 0.2 & 0.3 & 0.0 & 0.4 & 0.1 & 0.2 & 0.0 \\
\hline DBC & Neonate & Normal & 9 & 0.0 & 0.0 & 0.0 & 0.0 & 0.0 & 0.0 & 0.0 & 0.0 & 0.0 & 0.0 & 0.0 & 0.0 & 0.0 & 0.0 & 0.0 & 0.0 & 0.0 & 0.0 & 0.0 & 0.0 & 0.0 & 0.0 & 0.0 & 0.0 & 0.0 & 0.0 & 3.6 & 28.6 & 28.6 & $\begin{array}{ll}6 & 3.6\end{array}$ & 0.0 & 0.0 & 0.0 & 0.0 & 0.0 & 0.0 & 0.0 & 0.0 & 0.0 & 0.0 & 0.0 \\
\hline $\mathrm{BaP}$ & Neonate & Normal & 21 & 0.0 & 0.0 & 0.0 & 3.6 & 0.0 & 0.0 & 0.0 & 0.0 & 0.0 & 0.0 & 0.0 & 0.0 & 10.0 & 0.0 & 0.0 & 0.0 & 0.0 & 0.0 & 0.0 & 0.0 & 0.0 & 0.0 & 0.0 & 0.0 & 0.0 & 0.0 & 20.7 & 740.7 & 40.7 & 712.2 & 0.0 & 0.0 & 0.0 & 0.0 & 0.0 & 0.0 & 0.0 & 0.0 & 0.0 & 0.0 & 0.0 \\
\hline DBC & $15 \mathrm{wk}$ & Normal & 149 & 0.5 & 0.0 & 0.0 & 0.5 & 0.5 & 0.3 & 0.3 & 0.0 & 0.0 & 0.0 & 0.0 & 0.0 & 0.0 & 2.8 & 0.5 & 0.0 & 0.3 & 0.3 & 0.0 & 0.3 & 0.0 & 0.0 & 0.0 & 0.0 & 0.0 & 0.0 & 15.9 & 921.6 & 21.6 & 67.1 & 0.0 & 0.0 & 0.0 & 0.0 & 0.0 & 0.3 & 0.0 & 0.3 & 3.5 & 0.3 & 0.3 \\
\hline DBC & $25 \mathrm{wk}$ & Normal & 409 & 0.9 & 1.1 & 0.3 & 0.9 & 0.2 & 0.9 & 0.5 & 0.5 & 0.6 & 0.5 & 0.2 & 0.5 & 0.3 & 0.6 & 0.8 & 0.4 & 0.0 & 0.0 & 1.0 & 0.9 & 0.8 & 0.8 & 0.6 & 1.2 & 1.3 & 0.4 & 12.3 & 318.0 & 20.3 & 35.1 & 0.9 & 0.2 & 1.2 & 1.0 & 0.2 & 0.2 & 0.2 & 0.0 & 0.5 & 1.0 & 0.3 \\
\hline $3 \mathrm{C}$ & & & 324 & 0.1 & 0.8 & 0.0 & 0.0 & 0.3 & 0.4 & 0.0 & 2.0 & 1.1 & 0.0 & 0.6 & 0.0 & 0.0 & 1.4 & 0.8 & 0.0 & 0.0 & 0.1 & 3.6 & 0.1 & 1.5 & 0.0 & 1.1 & 0.1 & 0.5 & 0.0 & 10.6 & $\begin{array}{ll}69.9 \\
\end{array}$ & 11.4 & 46.6 & 0.0 & 0.0 & 0.9 & 0.3 & 0.0 & 0.0 & 0.1 & 0.0 & 0.1 & 0.1 & 0.0 \\
\hline DBC & $25 \mathrm{wk}$ & Tumor & 168 & 0.8 & 0.0 & 1.2 & 1.1 & 0.0 & 0.0 & 0.0 & 1.3 & 0.0 & 0.0 & 2.4 & 0.0 & 0.0 & 1.2 & 0.0 & 0.0 & 0.0 & 0.0 & 0.0 & 0.0 & 1.6 & 0.4 & 0.0 & 0.0 & 0.4 & 0.0 & 11.2 & 29.9 & 35.8 & 84.8 & 0.4 & 0.0 & 0.0 & 0.0 & 0.0 & 0.0 & 0.0 & 1.0 & 0.0 & 0.4 & 0.0 \\
\hline DBC & $35 \mathrm{wk}$ & Normal & 459 & 0.0 & 0.2 & 0.0 & 0.2 & 0.3 & 1.1 & 0.0 & 0.6 & 0.2 & 0.6 & 0.9 & 0.3 & 0.0 & 0.5 & 2.4 & 0.0 & 0.3 & 0.8 & 0.2 & 0.2 & 0.3 & 0.5 & 2.0 & 3.0 & 1.8 & 0.6 & 11.4 & 422.6 & 23.5 & $\begin{array}{lll}5 & 13.3\end{array}$ & 0.8 & 0.2 & 0.2 & 0.2 & 0.3 & 0.2 & 0.2 & 0.5 & 0.3 & 0.6 & 0.2 \\
\hline DBC & $35 \mathrm{wk}$ & AAH & 654 & 0.1 & 0.0 & 0.7 & 0.4 & 0.0 & 1.0 & 0.2 & 0.2 & 0.4 & 0.3 & 0.2 & 0.2 & 0.3 & 0.4 & 0.4 & 0.2 & 0.4 & 0.3 & 0.6 & 0.5 & 0.3 & 0.2 & 0.4 & 0.0 & 0.4 & 0.3 & 31.9 & 917.4 & 14.5 & 510.1 & 0.7 & 0.3 & 0.1 & 0.6 & 0.3 & 0.0 & 0.5 & 0.6 & 0.0 & 0.6 & 0.7 \\
\hline DBC & $35 \mathrm{wk}$ & Tumor & 830 & 0.6 & 0.2 & 0.0 & 0.8 & 0.3 & 0.9 & 0.4 & 0.7 & 0.7 & 0.3 & 0.0 & 0.3 & 0.2 & 0.3 & 0.5 & 0.1 & 2.5 & 0.1 & 0.3 & 0.8 & 0.3 & 0.2 & 0.3 & 0.0 & 0.2 & 1.0 & 22.1 & 140.2 & 37.6 & 620.5 & 1.0 & 0.6 & 1.4 & 0.3 & 0.2 & 0.0 & 1.8 & 0.1 & 0.5 & 0.6 & 0.4 \\
\hline DBC & $45 \mathrm{wk}$ & Normal & 1303 & 0.2 & 0.3 & 1.0 & 0.9 & 0.2 & 1.1 & 0.7 & 0.4 & 0.4 & 0.1 & 0.3 & 0.3 & 0.4 & 0.2 & 0.5 & 0.4 & 0.9 & 0.4 & 0.3 & 0.7 & 0.1 & 0.6 & 0.1 & 0.5 & 0.1 & 2.2 & 15.7 & 728.7 & 27.8 & 89.2 & 0.6 & 0.7 & 0.4 & 0.6 & 0.3 & 0.2 & 0.3 & 0.8 & 0.7 & 0.3 & 0.4 \\
\hline & $45 \mathrm{wk}$ & AAH & 392 & 0.7 & 0.3 & 0.6 & 0.3 & 0.5 & 0.0 & 0.5 & 0.6 & 0.3 & 0.3 & 0.3 & 0.6 & 2.4 & 0.0 & 9.7 & 0.3 & 0.3 & 0.3 & 1.0 & 0.6 & 0.0 & 0.0 & 0.3 & 0.5 & 0.0 & 0.0 & 22.7 & 737.4 & 32.5 & $\begin{array}{ll}5 & 18.0\end{array}$ & 0.6 & 1.6 & 0.3 & 0.6 & 0.0 & 0.3 & 1.5 & 1.2 & 0.3 & 0.0 & 8.0 \\
\hline$c$ & $\mathrm{wk}$ & imor & & & & & U. & & & S & 4.0 & & 0.3 & 0.3 & 0.2 & 0.1 & U. & & 0.3 & & 0 & & 0.2 & U.U & 0.1 & & U. & & & & & & & & & 0.2 & & & & 0.7 & & & & \\
\hline
\end{tabular}

Color key

$\begin{array}{lllllllllll}0.0 & 10.0 & 20.0 & 30.0 & 40.0 & 50.0 & 60.0 & 70.0 & 80.0 & 90.0 & 100.0\end{array}$

${ }^{a}$ Each heat map cell indicates the percentage of $\mathrm{CpG}$ sites positive for methylation. Blue corresponds to low methylation and red to high methylation according to color key shown below the table.

${ }^{b} \mathrm{CpG}$ site number specifies the specific base pair location of the $\mathrm{CpG}$ site in relation to the first base pair of the first exon.

${ }^{c}$ Number shown is the total number of reads associated with each amplicon for each sample. 
TABLE 4

Average Methylation per CpG Site for the Dapk1 Promoter Region Sequenced by Roche $454^{\text {a }}$

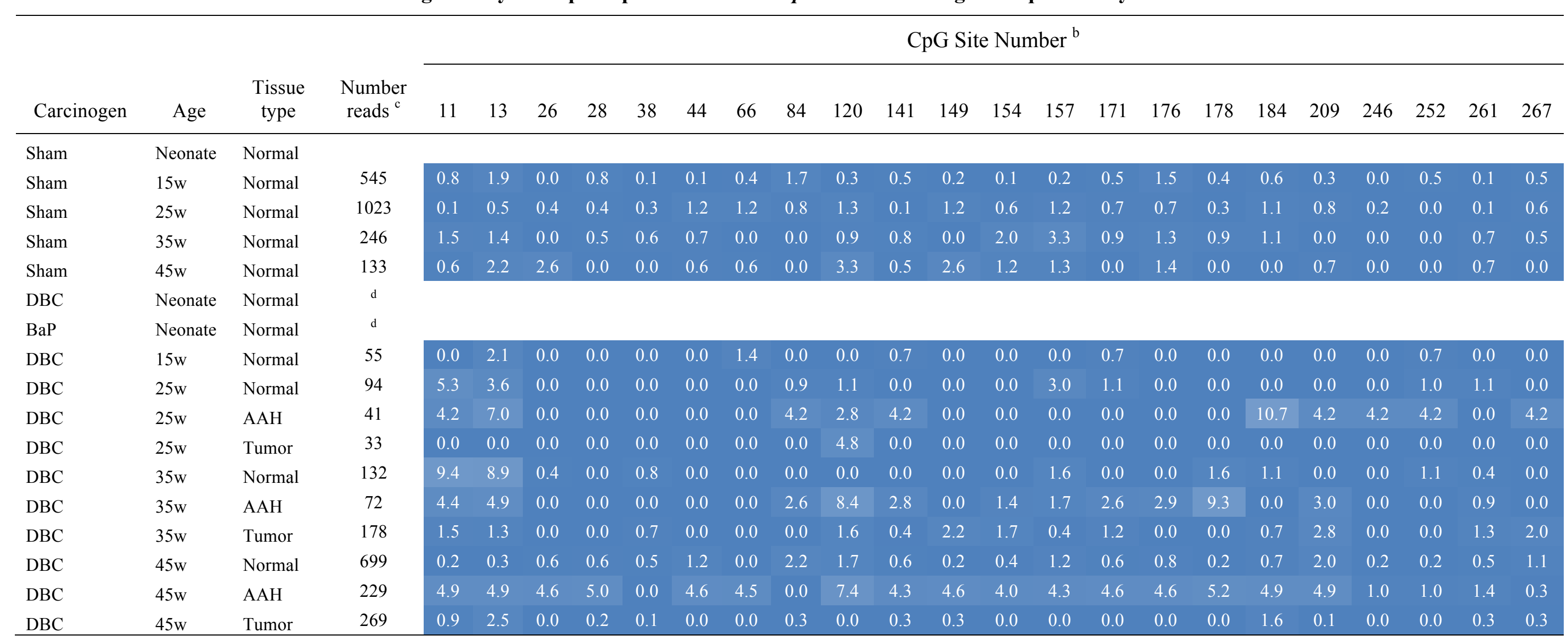

Color key

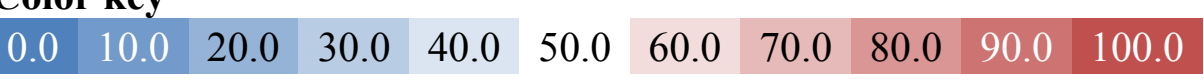

${ }^{a}$ Each heat map cell indicates the percentage of CpG sites positive for methylation. Blue corresponds to low methylation and red to high methylation according to color key shown below the table. ${ }^{6} \mathrm{CpG}$ site number specifies the specific base pair location of the $\mathrm{CpG}$ site in relation to the first base pair of the first exon.

Number shown is the total number of reads associated with each amplicon for each sample.

${ }^{d}$ Sequencing of this amplicon failed for the samples indicated. 
TABLE 5

Average Methylation per CpG Site for the Mgmt Promoter Region Sequenced by Roche $454^{\text {a }}$

\begin{tabular}{|c|c|c|c|c|c|c|c|c|c|c|c|c|c|c|c|c|c|c|c|c|c|c|c|}
\hline \multirow[b]{2}{*}{ Carcinogen } & \multirow[b]{2}{*}{ Age } & \multirow[b]{2}{*}{ Tissue type } & \multirow[b]{2}{*}{$\begin{array}{l}\text { Number } \\
\text { reads }^{c}\end{array}$} & \multicolumn{20}{|c|}{ CpG Site Number ${ }^{\mathrm{b}}$} \\
\hline & & & & 13 & 37 & 48 & 62 & 77 & 88 & 106 & 111 & 113 & 124 & 137 & 181 & 187 & 206 & 218 & 229 & 235 & 237 & 243 & 248 \\
\hline Sham & Neonate & Normal & 23 & 0.0 & 0.0 & 0.0 & 0.0 & 0.0 & 0.0 & 0.0 & 0.0 & 3.6 & 0.0 & 0.0 & 0.0 & 5.0 & 0.0 & 0.0 & 0.0 & 3.6 & 0.0 & 0.0 & 0.0 \\
\hline Sham & $15 \mathrm{w}$ & Normal & 950 & 0.0 & 1.7 & 4.6 & 1.2 & 0.0 & 1.0 & 1.2 & 0.2 & 0.4 & 0.8 & 1.3 & 0.2 & 1.8 & 0.3 & 1.1 & 1.3 & 1.9 & 0.9 & 1.2 & 1.3 \\
\hline Sham & $25 \mathrm{w}$ & Normal & 1239 & 1.4 & 0.8 & 1.1 & 0.8 & 1.7 & 1.2 & 1.1 & 1.0 & 1.4 & 1.1 & 0.6 & 1.3 & 0.8 & 0.9 & 0.5 & 0.4 & 1.2 & 1.3 & 1.5 & 1.1 \\
\hline Sham & $35 \mathrm{w}$ & Normal & 959 & 0.2 & 1.2 & 0.7 & 1.2 & 3.0 & 0.3 & 0.7 & 0.6 & 0.4 & 0.6 & 0.3 & 1.0 & 0.7 & 0.2 & 0.7 & 0.7 & 0.8 & 1.8 & 0.7 & 0.8 \\
\hline Sham & $45 \mathrm{w}$ & Normal & 996 & 0.3 & 0.5 & 0.1 & 0.1 & 0.8 & 1.3 & 0.4 & 0.7 & 0.8 & 0.9 & 0.4 & 0.8 & 0.8 & 0.5 & 0.7 & 0.7 & 1.0 & 1.2 & 0.7 & 0.5 \\
\hline DBC & Neonate & Normal & 72 & 1.1 & 0.0 & 1.1 & 0.0 & 0.0 & 0.0 & 1.1 & 0.0 & 1.9 & 1.1 & 4.2 & 0.0 & 0.0 & 1.3 & 0.0 & 0.7 & 1.1 & 0.0 & 0.0 & 0.0 \\
\hline $\mathrm{BaP}$ & Neonate & Normal & 61 & 0.0 & 0.0 & 0.0 & 2.9 & 0.0 & 1.1 & 1.1 & 1.1 & 1.1 & 1.1 & 2.3 & 2.3 & 1.1 & 1.1 & 1.1 & 2.5 & 1.1 & 2.7 & 5.3 & 2.3 \\
\hline DBC & $15 \mathrm{w}$ & Normal & 439 & 0.0 & 0.0 & 0.0 & 0.3 & 0.4 & 0.2 & 0.0 & 0.6 & 0.2 & 0.0 & 0.0 & 0.4 & 0.2 & 0.8 & 0.5 & 0.5 & 0.2 & 0.3 & 0.8 & 0.7 \\
\hline DBC & $25 \mathrm{w}$ & Normal & 1014 & 0.7 & 0.5 & 0.6 & 0.7 & 2.9 & 0.5 & 0.4 & 0.9 & 0.7 & 0.6 & 0.4 & 1.6 & 1.5 & 0.8 & 2.1 & 1.2 & 1.5 & 1.7 & 1.5 & 1.4 \\
\hline DBC & $25 \mathrm{w}$ & $\mathrm{AAH}$ & 621 & 3.5 & 4.6 & 3.4 & 5.0 & 8.0 & 3.8 & 5.1 & 4.1 & 5.5 & 2.9 & 3.0 & 6.9 & 5.6 & 3.7 & 6.1 & 3.7 & 6.5 & 6.8 & 6.7 & 5.8 \\
\hline DBC & $25 \mathrm{w}$ & Tumor & 446 & 2.9 & 3.5 & 4.9 & 4.6 & 6.8 & 3.9 & 4.4 & 4.6 & 4.2 & 3.7 & 0.4 & 4.6 & 4.3 & 1.5 & 1.8 & 4.3 & 4.9 & 4.4 & 4.2 & 5.5 \\
\hline DBC & $35 \mathrm{w}$ & Normal & 932 & 2.9 & 2.4 & 1.9 & 3.5 & 8.3 & 2.0 & 5.5 & 1.9 & 3.1 & 2.0 & 0.4 & 2.7 & 1.7 & 0.5 & 1.7 & 8.7 & 1.8 & 2.0 & 1.7 & 1.7 \\
\hline DBC & $35 \mathrm{w}$ & AAH & 827 & 1.7 & 4.3 & 2.3 & 6.2 & 4.8 & 2.3 & 2.2 & 1.8 & 4.8 & 3.1 & 1.1 & 0.8 & 0.7 & 0.9 & 0.8 & 0.6 & 0.6 & 1.2 & 2.0 & 2.0 \\
\hline DBC & $35 \mathrm{w}$ & Tumor & 1223 & 0.3 & 3.9 & 3.0 & 0.8 & 4.8 & 3.6 & 3.5 & 3.2 & 5.8 & 1.8 & 1.6 & 3.8 & 2.3 & 1.3 & 1.2 & 1.8 & 1.6 & 0.9 & 3.2 & 3.4 \\
\hline DBC & $45 \mathrm{w}$ & Normal & 1183 & 0.2 & 0.4 & 0.3 & 0.6 & 0.7 & 0.9 & 1.5 & 1.0 & 2.2 & 1.1 & 0.3 & 0.8 & 0.7 & 0.8 & 0.5 & 0.4 & 0.5 & 1.2 & 1.3 & 0.8 \\
\hline DBC & $45 \mathrm{w}$ & AAH & 1115 & 0.2 & 1.8 & 1.0 & 0.4 & 1.2 & 0.5 & 8.8 & 0.5 & 0.3 & 0.2 & 0.3 & 0.3 & 0.5 & 0.3 & 0.6 & 0.4 & 0.1 & 5.4 & 0.3 & 0.4 \\
\hline DBC & $45 \mathrm{w}$ & Tumor & 1180 & 11.1 & 12.2 & 11.8 & 10.6 & 3.3 & 7.8 & 7.3 & 7.4 & 10.7 & 2.6 & 6.1 & 4.0 & 3.4 & 0.3 & 2.3 & 2.3 & 1.4 & 3.3 & 9.4 & 8.5 \\
\hline
\end{tabular}

\section{Color key}

$\begin{array}{llllllllllll}0.0 & 10.0 & 20.0 & 30.0 & 40.0 & 50.0 & 60.0 & 70.0 & 80.0 & 90.0 & 100.0\end{array}$

${ }^{a}$ Each heat map cell indicates the percentage of $\mathrm{CpG}$ sites positive for methylation. Blue corresponds to low methylation and red to high methylation according to color key shown below the table. ${ }^{\mathrm{b}} \mathrm{CpG}$ site number specifies the specific base pair location of the $\mathrm{CpG}$ site in relation to the first base pair of the first exon.

$\mathrm{CpG}$ site number specifies the specific base pair location of the $\mathrm{CpG}$ site in relation to the first
${ }^{c}$ Number shown is the total number of reads associated with each amplicon for each sample. 
TABLE 6

Average Methylation per CpG Site for the Rarb Promoter Region Sequenced by Roche $454^{\text {a }}$

\begin{tabular}{|c|c|c|c|c|c|c|c|c|c|c|c|c|c|c|c|c|c|c|c|c|}
\hline \multirow[b]{2}{*}{ Carcinogen } & \multirow[b]{2}{*}{ Age } & \multirow[b]{2}{*}{ Tissue type } & \multirow[b]{2}{*}{$\begin{array}{l}\text { Number } \\
\text { reads }^{c}\end{array}$} & \multicolumn{17}{|c|}{ CpG Site Number ${ }^{\mathrm{b}}$} \\
\hline & & & & 16 & 27 & 48 & 52 & 63 & 70 & 76 & 84 & 96 & 116 & 124 & 136 & 138 & 140 & 148 & 150 & 156 \\
\hline Sham & Neonate & Normal & 146 & 0.5 & 1.3 & 0.0 & 0.0 & 2.0 & 0.7 & 0.0 & 0.0 & 0.5 & 0.0 & 0.0 & 0.7 & 0.5 & 0.0 & 0.0 & 1.3 & 0.0 \\
\hline Sham & $15 \mathrm{w}$ & Normal & 1520 & 4.2 & 3.4 & 0.8 & 0.8 & 0.9 & 0.6 & 1.8 & 0.7 & 0.5 & 0.4 & 0.1 & 0.0 & 0.2 & 0.7 & 0.7 & 0.7 & 0.3 \\
\hline Sham & $25 \mathrm{w}$ & Normal & 1557 & 0.5 & 0.7 & 0.2 & 0.7 & 1.2 & 0.9 & 0.6 & 1.7 & 0.7 & 0.6 & 0.4 & 1.0 & 0.7 & 0.9 & 0.2 & 0.2 & 0.6 \\
\hline Sham & $35 \mathrm{w}$ & Normal & 1507 & 7.0 & 8.1 & 0.5 & 1.1 & 2.6 & 0.6 & 1.0 & 0.5 & 0.7 & 1.4 & 0.5 & 0.9 & 0.6 & 0.9 & 0.4 & 0.5 & 0.5 \\
\hline Sham & $45 \mathrm{w}$ & Normal & 1510 & 9.6 & 5.3 & 0.6 & 0.9 & 1.5 & 0.5 & 0.7 & 0.6 & 0.6 & 0.6 & 1.1 & 0.5 & 0.3 & 0.6 & 0.7 & 0.3 & 0.9 \\
\hline DBC & Neonate & Normal & 206 & 0.0 & 0.9 & 0.7 & 0.0 & 0.8 & 0.0 & 0.7 & 1.4 & 0.7 & 0.7 & 0.3 & 0.3 & 1.4 & 0.5 & 0.3 & 0.5 & 0.5 \\
\hline $\mathrm{BaP}$ & Neonate & Normal & 269 & 0.2 & 1.1 & 0.0 & 0.0 & 0.7 & 0.2 & 0.0 & 1.1 & 1.2 & 0.9 & 0.9 & 2.0 & 2.1 & 0.0 & 0.0 & 0.2 & 0.7 \\
\hline DBC & $15 \mathrm{w}$ & Normal & 1079 & 3.5 & 2.9 & 1.0 & 1.3 & 1.8 & 0.8 & 0.5 & 1.1 & 0.4 & 0.4 & 0.3 & 0.4 & 0.4 & 0.4 & 0.6 & 0.3 & 0.3 \\
\hline DBC & $25 \mathrm{w}$ & Normal & 1427 & 5.1 & 8.4 & 1.4 & 2.1 & 2.5 & 1.6 & 1.2 & 1.4 & 1.5 & 0.5 & 0.7 & 0.9 & 0.7 & 0.7 & 1.0 & 0.4 & 0.8 \\
\hline DBC & $25 \mathrm{w}$ & AAH & 1434 & 10.1 & 7.9 & 3.9 & 4.7 & 4.6 & 3.5 & 2.1 & 2.3 & 3.4 & 4.7 & 1.6 & 1.7 & 0.8 & 1.3 & 1.5 & 0.3 & 1.0 \\
\hline DBC & $25 \mathrm{w}$ & Tumor & 1124 & 4.1 & 2.1 & 0.3 & 5.8 & 8.7 & 1.0 & 5.3 & 1.0 & 0.8 & 0.9 & 0.4 & 0.4 & 1.0 & 2.8 & 0.7 & 0.4 & 1.0 \\
\hline DBC & $35 \mathrm{w}$ & Normal & 1473 & 7.5 & 5.0 & 1.1 & 1.1 & 1.5 & 1.0 & 0.6 & 0.6 & 0.6 & 1.1 & 0.3 & 1.0 & 0.8 & 0.4 & 0.9 & 0.3 & 1.6 \\
\hline DBC & $35 \mathrm{w}$ & AAH & 1079 & 7.3 & 8.9 & 0.9 & 2.9 & 4.0 & 2.6 & 1.6 & 0.1 & 1.1 & 0.6 & 0.6 & 1.9 & 1.5 & 1.3 & 1.0 & 1.3 & 0.7 \\
\hline DBC & $35 \mathrm{w}$ & Tumor & 1531 & 14.3 & 1.1 & 1.3 & 2.9 & 3.2 & 1.5 & 1.2 & 0.4 & 0.7 & 1.1 & 0.6 & 0.4 & 0.2 & 0.5 & 0.8 & 0.5 & 0.4 \\
\hline DBC & $45 \mathrm{w}$ & Normal & 1456 & 4.6 & 0.2 & 0.6 & 1.8 & 1.6 & 0.7 & 1.1 & 0.5 & 0.5 & 0.8 & 0.9 & 0.4 & 0.4 & 0.4 & 0.6 & 0.8 & 0.5 \\
\hline DBC & $45 \mathrm{w}$ & AAH & 1463 & 1.5 & 2.8 & 0.9 & 0.3 & 2.2 & 0.6 & 1.1 & 1.2 & 0.3 & 0.6 & 0.2 & 0.4 & 0.4 & 1.1 & 1.1 & 0.1 & 0.1 \\
\hline DBC & $45 w$ & Tumor & 1486 & 8.0 & 0.4 & 0.9 & 2.6 & 1.3 & 2.0 & 1.4 & 0.2 & 0.9 & 0.6 & 0.9 & 1.7 & 0.4 & 0.7 & 0.7 & 0.8 & 0.2 \\
\hline
\end{tabular}

Color key

$\begin{array}{llllllllllll}0.0 & 10.0 & 20.0 & 30.0 & 40.0 & 50.0 & 60.0 & 70.0 & 80.0 & 90.0 & 100.0\end{array}$

${ }^{a}$ Each heat map cell indicates the percentage of $\mathrm{CpG}$ sites positive for methylation. Blue corresponds to low methylation and red to high methylation according to color key shown below the table ${ }^{\mathrm{b}} \mathrm{CpG}$ site number specifies the specific base pair location of the CpG site in relation to the first base pair of the first exon.

${ }^{c}$ Number shown is the total number of reads associated with each amplicon for each sample. 
Overall, methylation of $\mathrm{CpG}$ sites in the amplicon sequenced for $C d k n 2 a$ was minimal $(<10 \%)$, with one region from sites 27 to 30 showing a moderate methylation frequency (15 to $60 \%$ ) (Fig. 8). Results of hierarchical clustering according to animal group showed that sham mice had a similar methylation profile, with the exception of 25 week-old sham mice, which grouped more closely with DBC-initiated offspring (Fig. 8A). When considering each individual mouse methylation pattern, no distinct clusters were evident, likely due to the broad pattern of overall very low methylation evident across all samples for most $\mathrm{CpG}$ sites (Fig. 8B).

Analysis of methylation for Dapkl (Fig. 9) revealed that the region sequenced is consistently unmethylated in lung tissues from all ages of offspring, with no discernible differences among sham, DBC- or BaP-initiated mice. Cluster analysis did not reveal any pattern in methylation for age, carcinogen treatment or tissue type for average methylation data (Fig. 9A). Small groupings of two to three animals by tissue type, such as 35 week DBC-initiated mice were observed in the bi-directional hierarchical clustering of the individual mouse methylation profiles (Fig. 9B). Results for methylation analyses of the Mgmt and Rarb amplicons was similar to that for Dapkl in that the frequency of methylation was very low for all the $\mathrm{CpG}$ sites examined, and no clear patterns emerged from the unsupervised clustering according to offspring age, carcinogen exposure or the type of lung tissue (Fig. 10 and 11). 
FIG. 8. Methylation profile for region of $C d k n 2 a$ gene promoter by 454 deep bisulfite sequencing. Each heat map cell indicates the percentage of $\mathrm{CpG}$ sites positive for methylation. Heat map shading indicates percent methylation for each $\mathrm{CpG}$ site (scale bar blue to red shown at bottom). (A) Bi-directional hierarchical clustering of the average methylation of groups for each time point ( $n=4$ mice per group). Green bars indicate sample groupings for lung tissues from sham-initiated offspring, neonates initiated with $\mathrm{DBC}$ and $\mathrm{BaP}$ and offspring initiated with $\mathrm{DBC}$ at multiple time points. (B) Bidirectional hierarchical clustering of each sampled mouse individually with mice labeled A-D for each time, tissue type. Colored boxes indicate grouping per animal for each time point and tissue type. $\mathrm{N}$, normal; $\mathrm{H}$, hyperplasia; $\mathrm{T}$, tumor; Neo, neonate. 


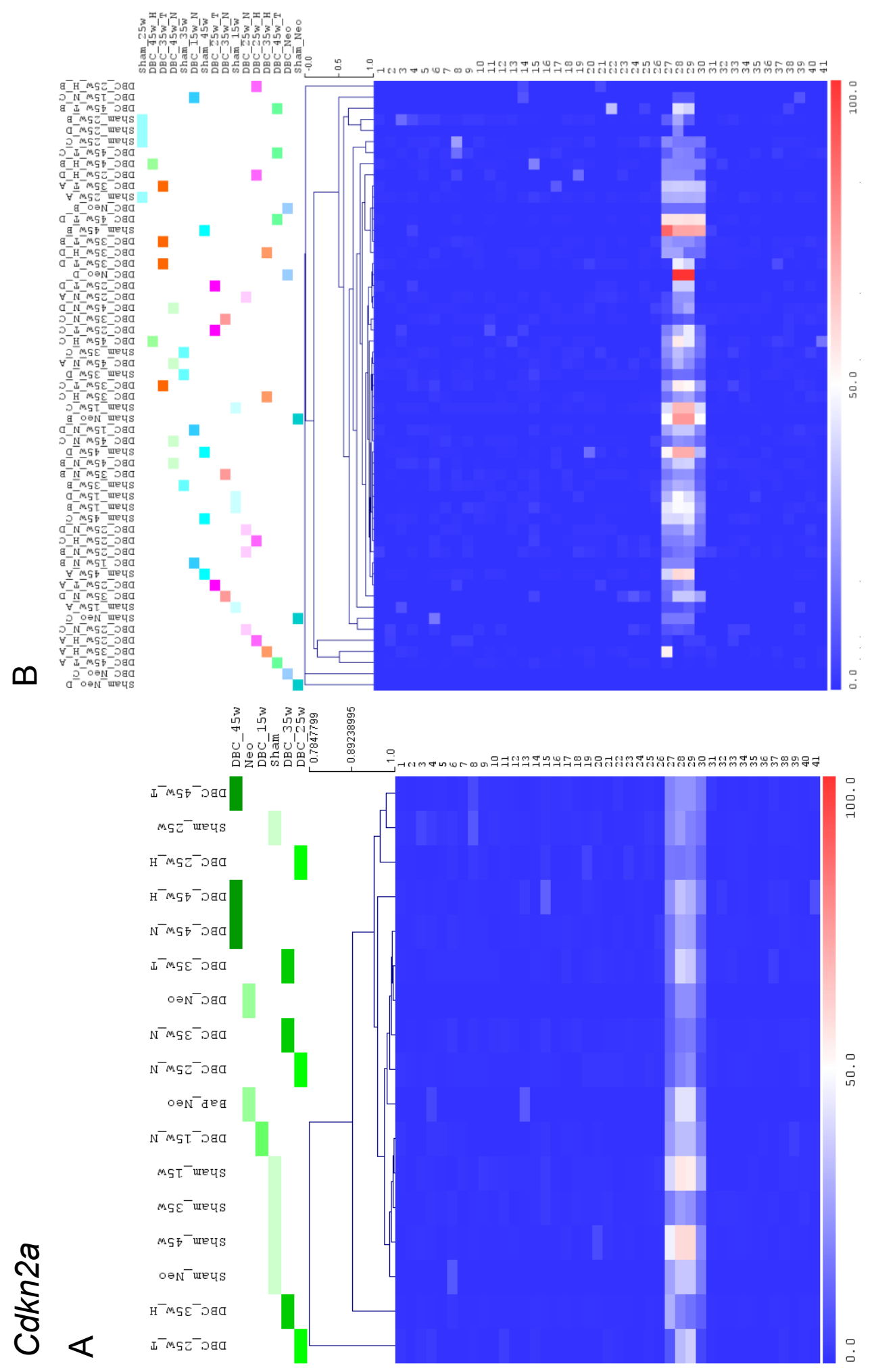


FIG. 9. Methylation profile for region of Dapkl gene promoter by 454 deep bisulfite sequencing. Each heat map cell indicates the percentage of $\mathrm{CpG}$ sites positive for methylation. Heat map shading indicates percent methylation for each $\mathrm{CpG}$ site (scale bar blue to red shown at bottom). (A) Bi-directional hierarchical clustering of the average methylation of groups for each time point ( $n=4$ mice per group). Green bars indicate sample groupings for lung tissues from sham-initiated offspring and offspring initiated with DBC at multiple time points. (B) Bi-directional hierarchical clustering of each sampled mouse individually with mice labeled A-D for each time, tissue type. Colored boxes indicate grouping per animal for each time point and tissue type. $\mathrm{N}$, normal; $\mathrm{H}$, hyperplasia; T, tumor; Neo, neonate. 


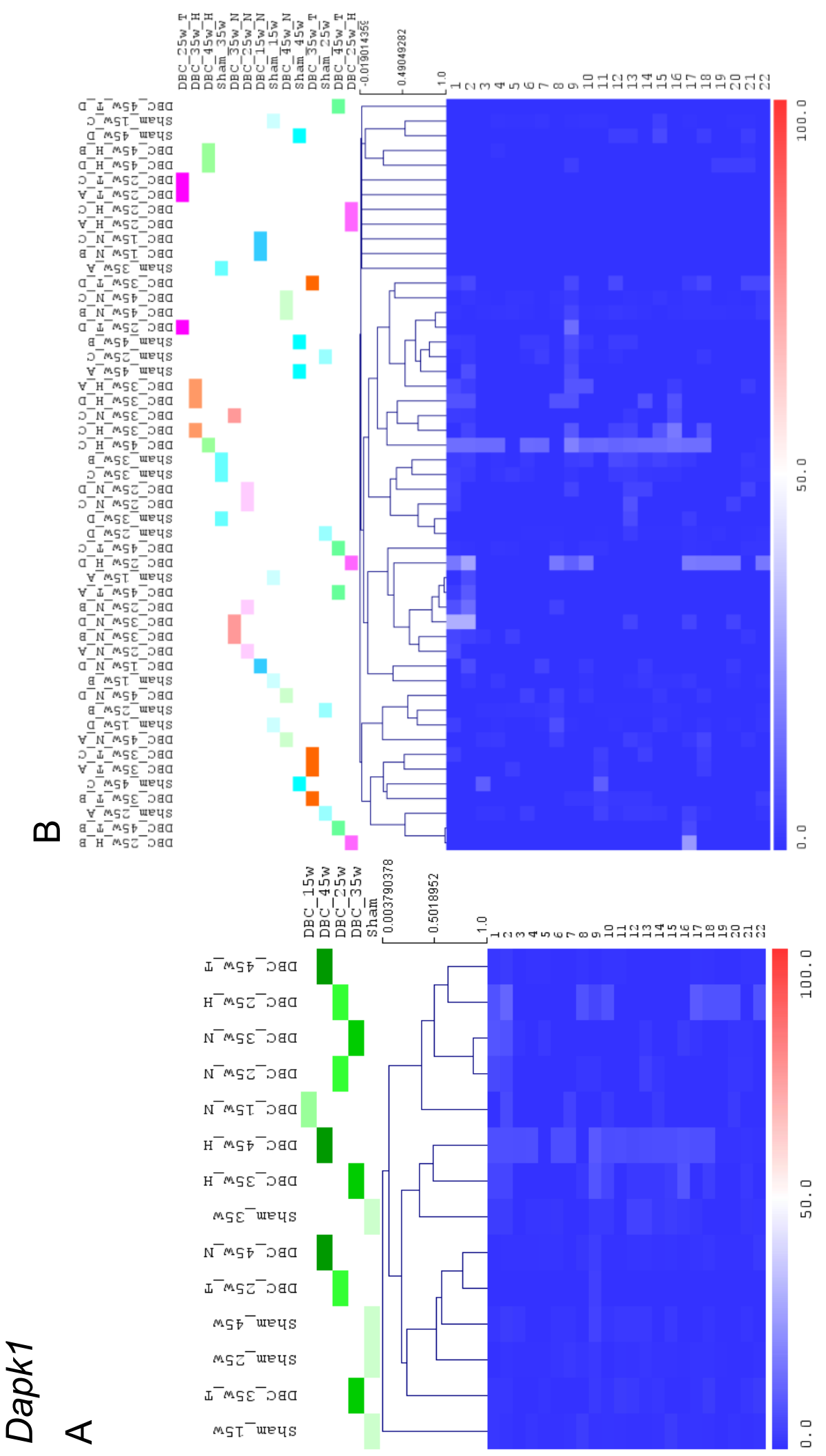


FIG. 10. Methylation profile for region of $M g m t$ gene promoter by 454 deep bisulfite sequencing. Each heat map cell indicates the percentage of $\mathrm{CpG}$ sites positive for methylation. Heat map shading indicates percent methylation for each $\mathrm{CpG}$ site (scale bar blue to red shown at bottom). (A) Bi-directional hierarchical clustering of the average methylation of groups for each time point ( $n=4$ mice per group). Green bars indicate sample groupings for lung tissues from sham-initiated offspring, neonates initiated with $\mathrm{DBC}$ and $\mathrm{BaP}$ and offspring initiated with $\mathrm{DBC}$ at multiple time points. (B) Bidirectional hierarchical clustering of each sampled mouse individually with mice labeled A-D for each time, tissue type. Colored boxes indicate grouping per animal for each time point and tissue type. N, normal; $\mathrm{H}$, hyperplasia; $\mathrm{T}$, tumor; Neo, neonate. 


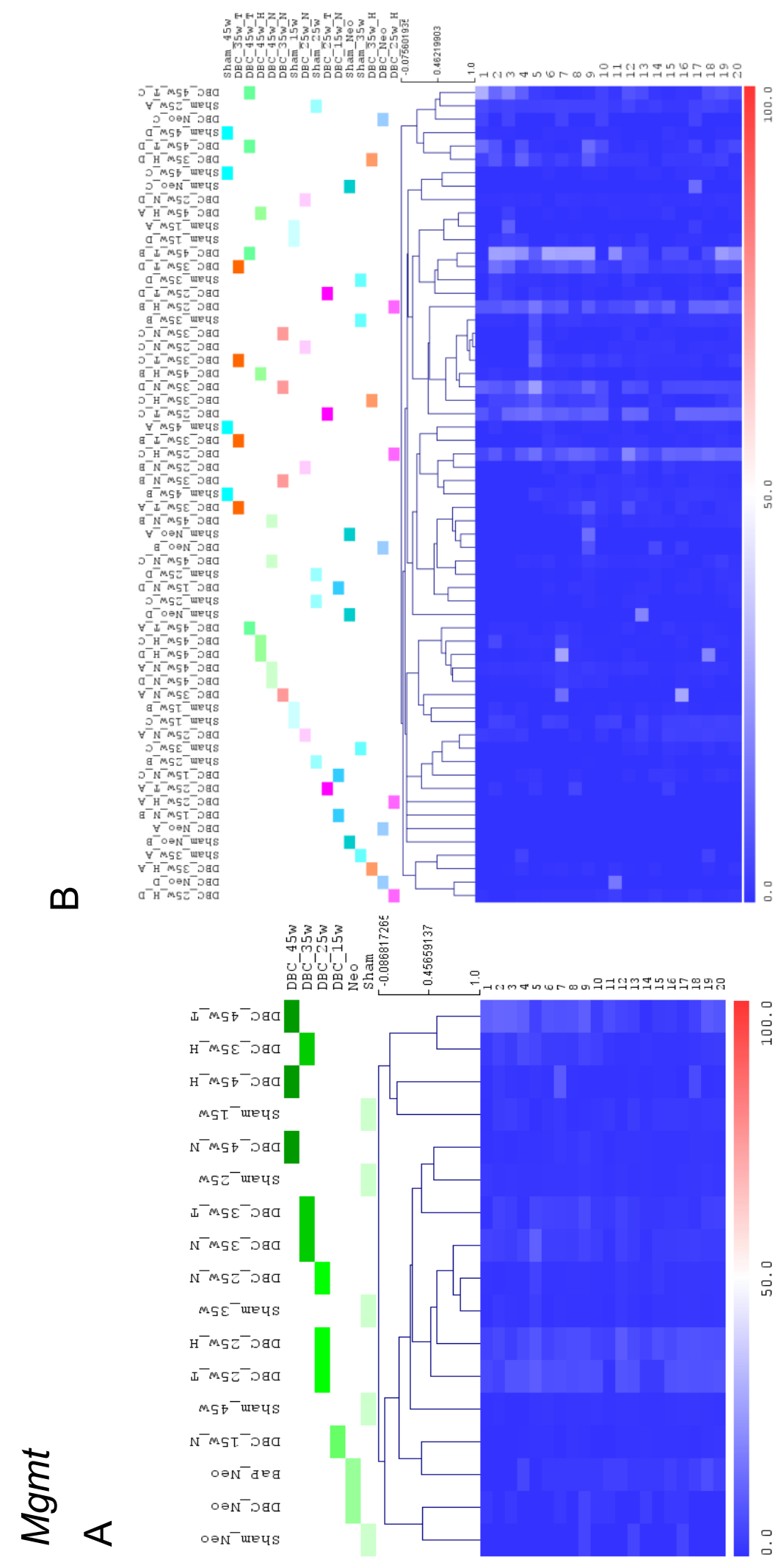


FIG. 11. Methylation profile for region of Rarb gene promoter by 454 deep bisulfite sequencing. Each heat map cell indicates the percentage of $\mathrm{CpG}$ sites positive for methylation. Heat map shading indicates percent methylation for each $\mathrm{CpG}$ site (scale bar blue to red shown at bottom). (A) Bi-directional hierarchical clustering of the average methylation of groups for each time point ( $n=4$ mice per group). Green bars indicate sample groupings for lung tissues from sham-initiated offspring, neonates initiated with $\mathrm{DBC}$ and $\mathrm{BaP}$ and offspring initiated with $\mathrm{DBC}$ at multiple time points. (B) Bidirectional hierarchical clustering of each sampled mouse individually with mice labeled A-D for each time, tissue type. Colored boxes indicate grouping per animal for each time point and tissue type. $\mathrm{N}$, normal; $\mathrm{H}$, hyperplasia; $\mathrm{T}$, tumor; Neo, neonate. 

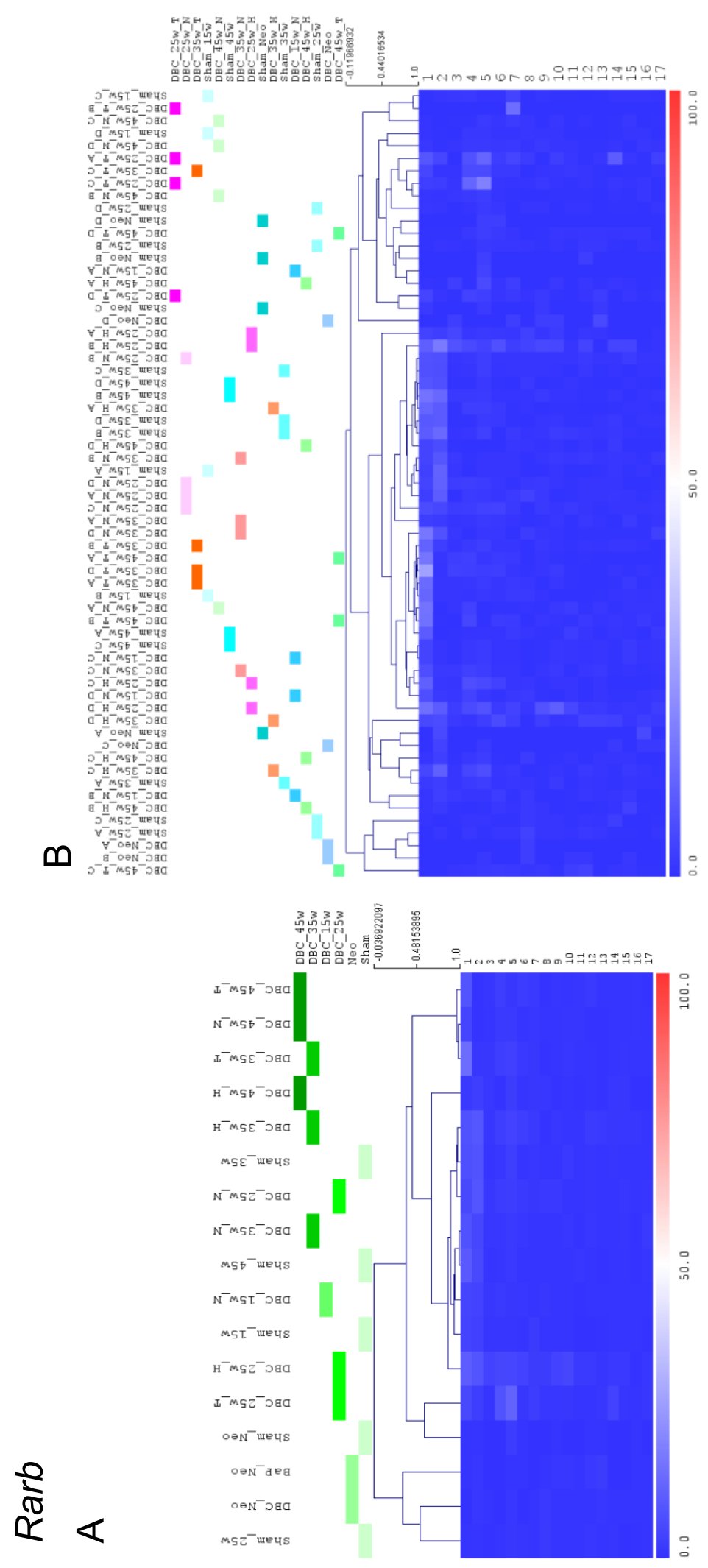
Genome-Wide Methylation Analysis

The impact of transplacental exposure to DBC and BaP on DNA methylation of lung tumors and normal adjacent lung tissue in the adult offspring mice was assessed utilizing NimbleGen's genome-wide DNA methylation promoter array. The processed array data obtained from NimbleGen are provided as calculated peak scores for all methylation peaks detected on the array (Appendix A) (Fish, 2015a) and calculated peak scores for peaks nearest to transcription start site (Appendix B) (Fish, 2015b). Given the small sample size of three in individuals per tissue type, the large number of observations and the limited range of values for peak scores, we did not expect to detect significant differences among the treatment groups using standard statistical analysis for microarrays. Thus, we employed a gene ontology analytical approach to determine whether differences were evident among the biological pathways represented by differentially methylated genes for the various lung tissue types examined, including: normal sham, normal adjacent in DBC-initiated offspring (AdjDBC), tumor in DBCinitiated offspring (TumDBC), normal adjacent in BaP-initiated offspring (AdjBaP), and tumor in BaP-initiated offspring (TumBaP). A summary table of peak scores for all genes identified as methylated in any sample is provided in Table C.1 of Apendix C (Fish, 2015c), which was generated using the processed data for nearest peak scores. This summary table was then used to generate gene lists with unique terms (e.g., methylated in sham tissues but not in AdjDBC or TumDBC tissues) for further analysis. Gene lists for all comparisons of interest are provided in Table E.2 of Appendix D (Fish, 2015d). 

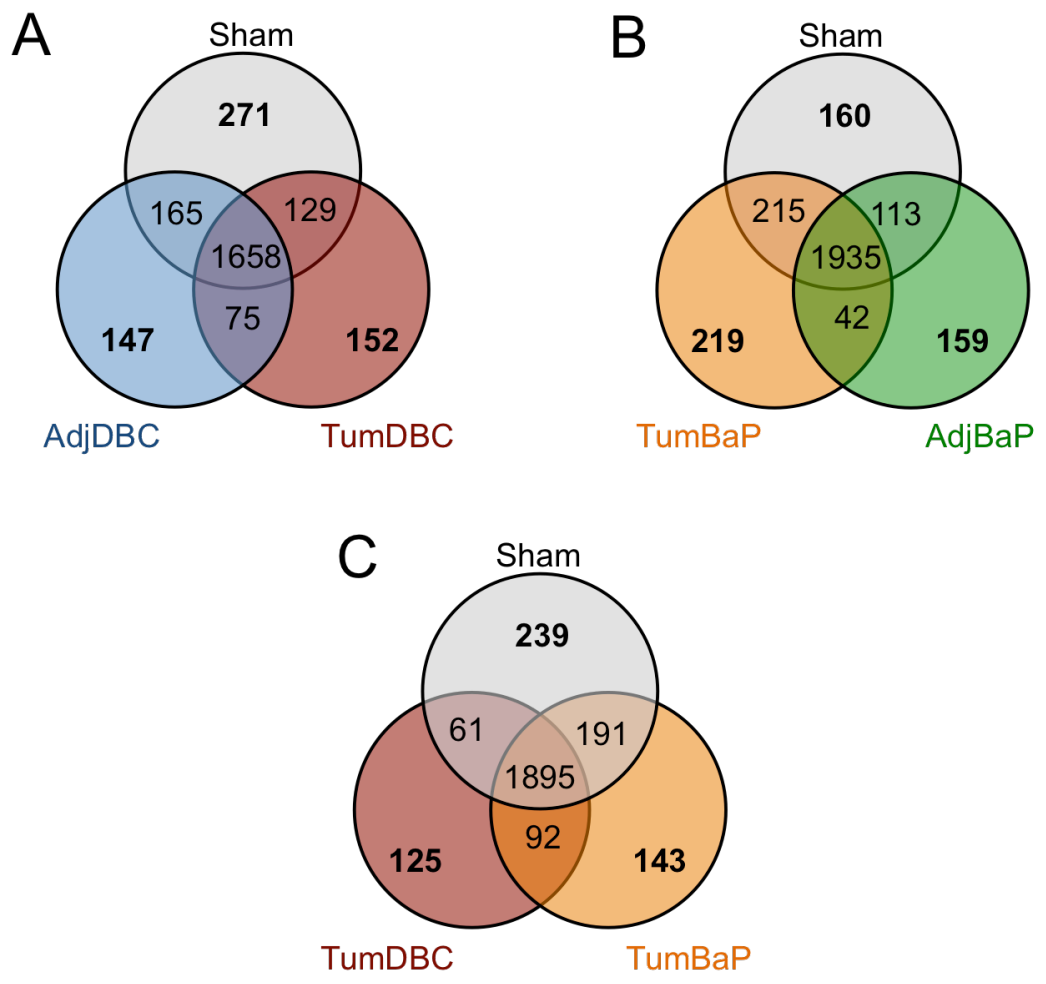

FIG. 12. Venn diagrams depicting segregation of differentially methylated genes among lung tissue types, as well as the number of genes similarly methylated among groups (overlapping regions). (A) Venn diagram depicts the number of genes identified as methylated for sham normal lung tissue, normal adjacent (AdjDBC) and tumor tissue (TumDBC) from DBC-initiated offspring. (B) Venn diagram depicts the number of genes identified as methylated for sham normal lung tissue, normal adjacent (AdjBaP)and tumor tissue (TumBaP) from BaP-initiated offspring. (C) Venn diagram depicts the number of genes identified as methylated for sham normal lung tissue and tumor tissues from both $\mathrm{BaP}$ - and $\mathrm{DBC}$-initiated offspring. Coloring of Venn diagrams corresponds to colors assigned to tissue types in Tables 7-9.

Functional gene ontology (GO) analysis of differentially methylated genes for adjacent and tumor tissue of lungs from $\mathrm{DBC}$ - or BaP-initiated mice and from shaminitiated mice revealed differences in the GO terms for biological processes associated with each group. Each of the Venn diagrams (Fig. 12) depicts the number of differentially methylated genes identified for each sample type used in the functional gene ontology 
analysis, as well as the number of genes similarly methylated among groups (overlapping regions). This GO analysis, based on the differentially methylated gene lists of a given comparison, reports what functions are associated with genes methylated in a select tissue sample. For example, the analysis identifies GO terms associated with genes methylated in sham tissues, but not methylated in tumor DBC or adjacent DBC tissues. Results of gene ontology analysis are presented here for biological process terms only; see Tables E.1-E.3 (Appendix E) for complete results of ontology analysis, including GO terms and FDR values for biological process, cellular compartment and molecular function.

By populating the gene list for ontology analysis with genes that were uniquely methylated in specific sample types (e.g., normal tissue from sham-initiated mice) or groupings of samples (e.g., genes methylated in tumors from BaP-and DBC-initiated mice), we were able to identify biological functions associated with promoter hypermethylation specific to a tissue type. Table 7 and Fig. 13 depict functional gene ontology analysis of differently methylated genes for adjacent and tumor tissues of lungs from DBC-initiated mice and from normal lung tissue of sham-initiated mice. A total of 44 categories are represented by the comparison of the differentially methylated gene lists. Genes in the methylated Sham list are represented in 40 of the 44 enriched GO terms, while the methylated tumor DBC gene list is composed of 35 enriched GO terms. Interestingly, there are only four enriched GO terms associated with the methylated genes in the normal adjacent DBC tissue samples. The strength of enrichment is indicated by the FDR-adjusted $p$-value. While 31 terms appear in both Sham and TumDBC enriched GO term lists, it is important to note that genes that made up the comparison lists for 
FIG. 13. Biological processes associated with genes differentially methylated in lung tumors from both DBC- and BaP-initiated offspring. Gene ontology map for GO terms representing hypermethylated gene promoters unique to lung tumor tissues from DBCand $\mathrm{BaP}$-initiated offspring (not sham). Gene ontology analysis was performed using the AgriGO singular enrichment analysis (SEA) tool (http://bioinfo.cau.edu.cn/agriGO) against the mouse gene ontology database (Mouse Genome Informatics). Significantly enriched GO terms (Generic GO Slim) were determined using the Fisher test with an FDR-corrected significance level of $P<0.05$ (Yekutieli multi-test adjustment method) and the minimum number of mapping entries set at 5 genes. 


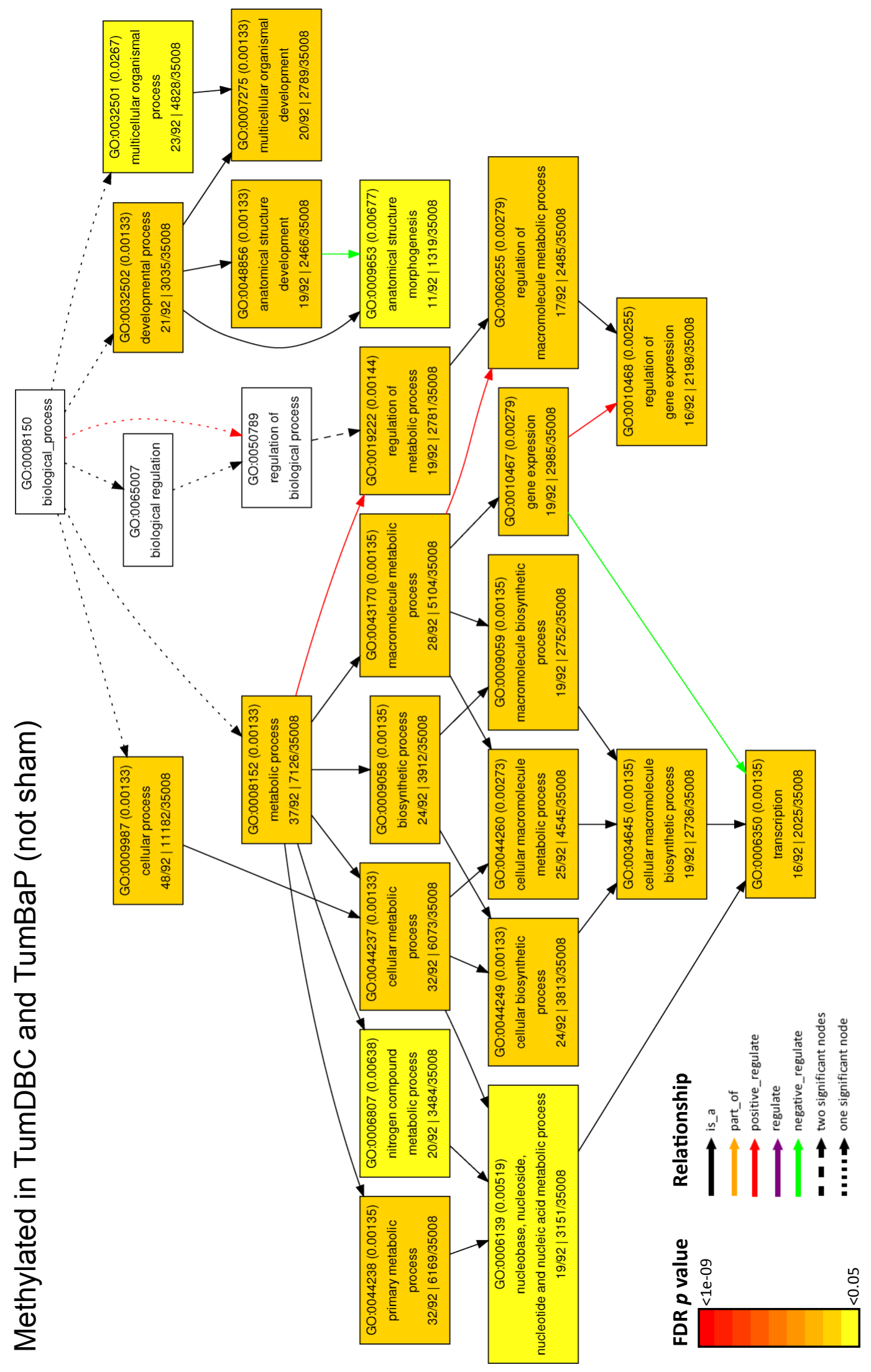




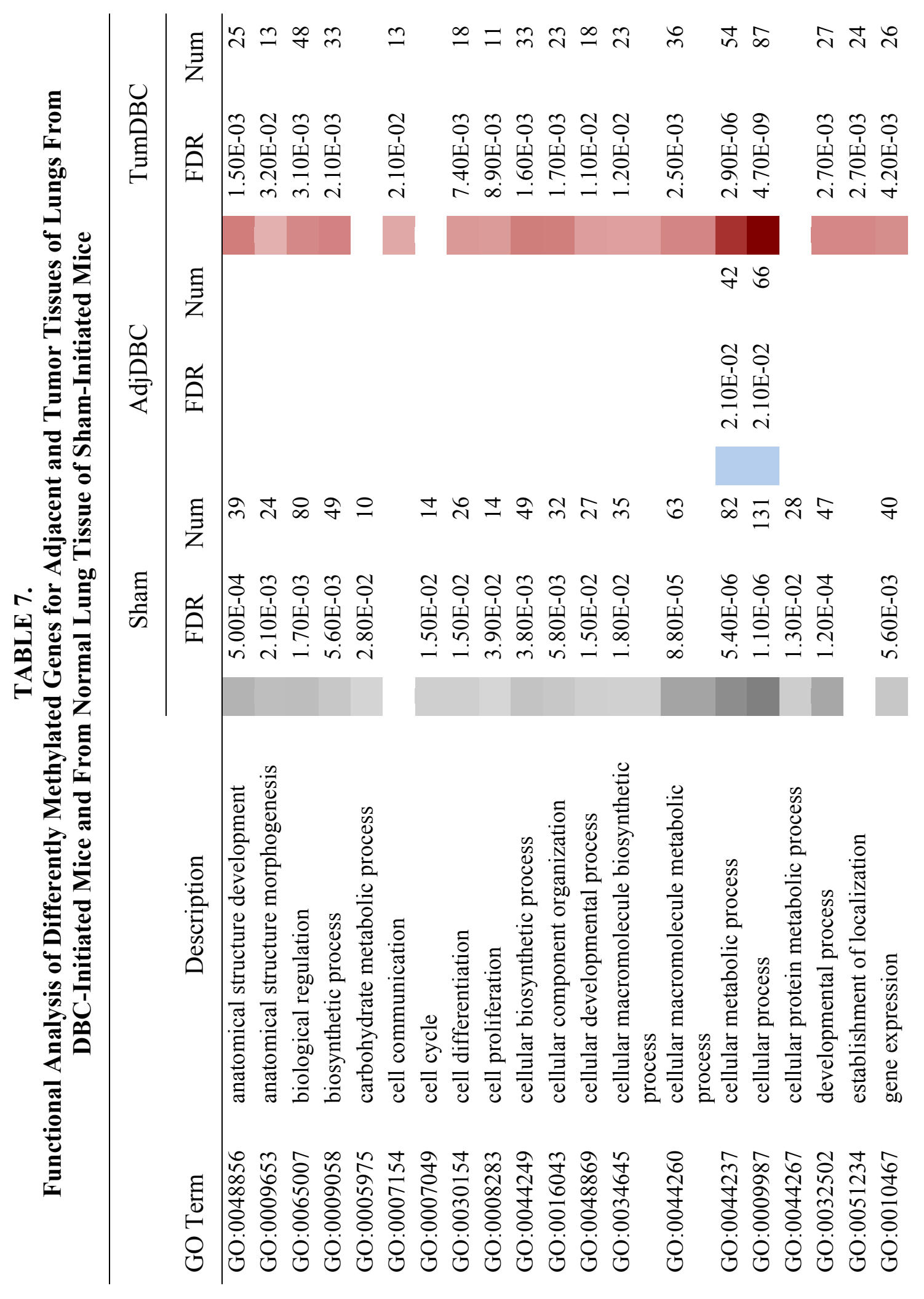




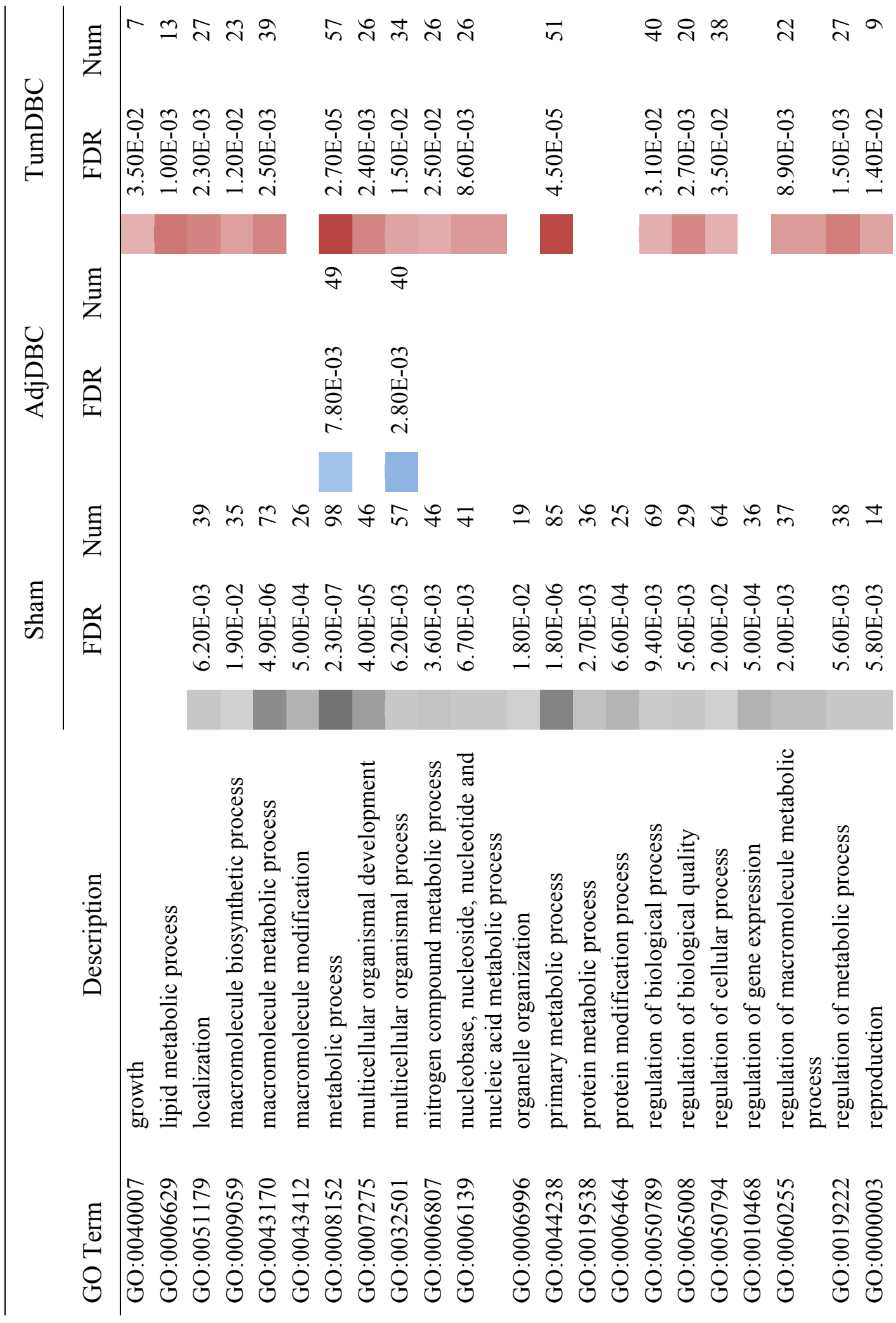




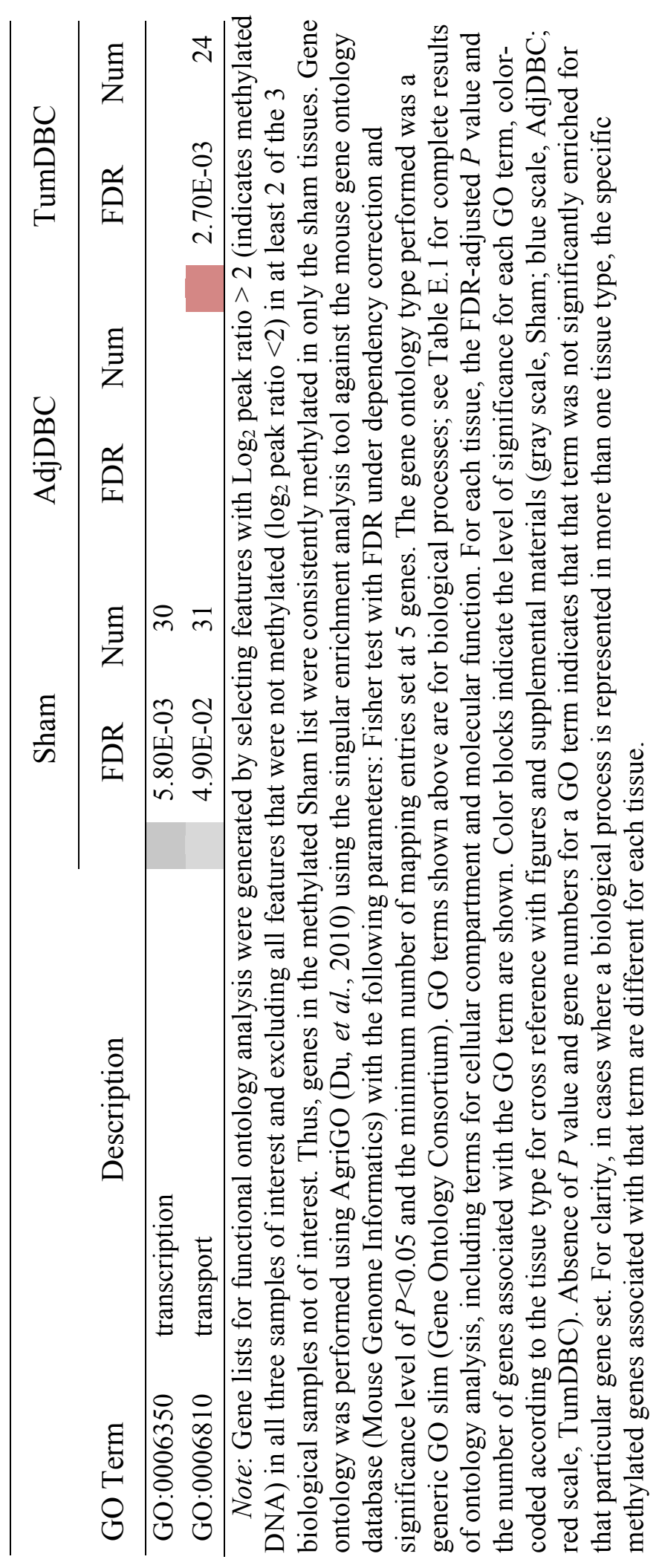


performing the GO analysis were entirely unique. Furthermore, this analysis does not discern the regulatory function of the genes in the lists, but can facilitate the exploration of biological meaning in higher order pathways and the inter-relationships between terms. Within this comparison (Sham vs. AdjDBC and TumDBC), methylated genes were overrepresented in biological function categories unique to normal lung tissue of shaminitiated mice include cell cycle $(\mathrm{FDR}=1.50 \mathrm{E}-02)$, regulation of gene expression $(\mathrm{FDR}=$ $5.00 \mathrm{E}-04)$, transcription $(\mathrm{FDR}=5.80 \mathrm{E}-03)$ and protein modification process $(\mathrm{FDR}=$ 6.60E-04), among others. Genes methylated in tumor tissue from DBC-initiated mice were over-represented in biological function categories cell communication (FDR = $2.10 \mathrm{E}-02)$ and growth $(\mathrm{FDR}=3.50 \mathrm{E}-02)$.

Fig. 14 and Table 8 show the results of functional analysis of differently methylated genes for adjacent and tumor tissues of lungs from BaP-initiated mice and from normal lung tissue of sham-initiated mice. A total of 40 enriched GO terms are represented, of which 36 are associated with the genes methylated in tumor BaP samples, 20 are associated with genes methylated in normal adjacent BaP tissue, and only 6 GO terms are over-represented from genes methylated in normal lung tissue of sham-initiated mice. The higher number of enriched categories for tumor $\mathrm{BaP}$ suggests that the methylation profile associated with tumor $\mathrm{BaP}$ is more functionally diverse than that of either normal adjacent $\mathrm{BaP}$ or Sham profiles. Tumor tissue from BaP-initiated mice yielded a methylation profile enriched for genes associated with cell-cell signaling (FDR $=1.10 \mathrm{E}-02)$, cellular developmental process $(\mathrm{FDR}=4.0 \mathrm{E}-03)$ and translation $(\mathrm{FDR}=$ 
Methylated in Sham (not TumDBC or TumBaP)

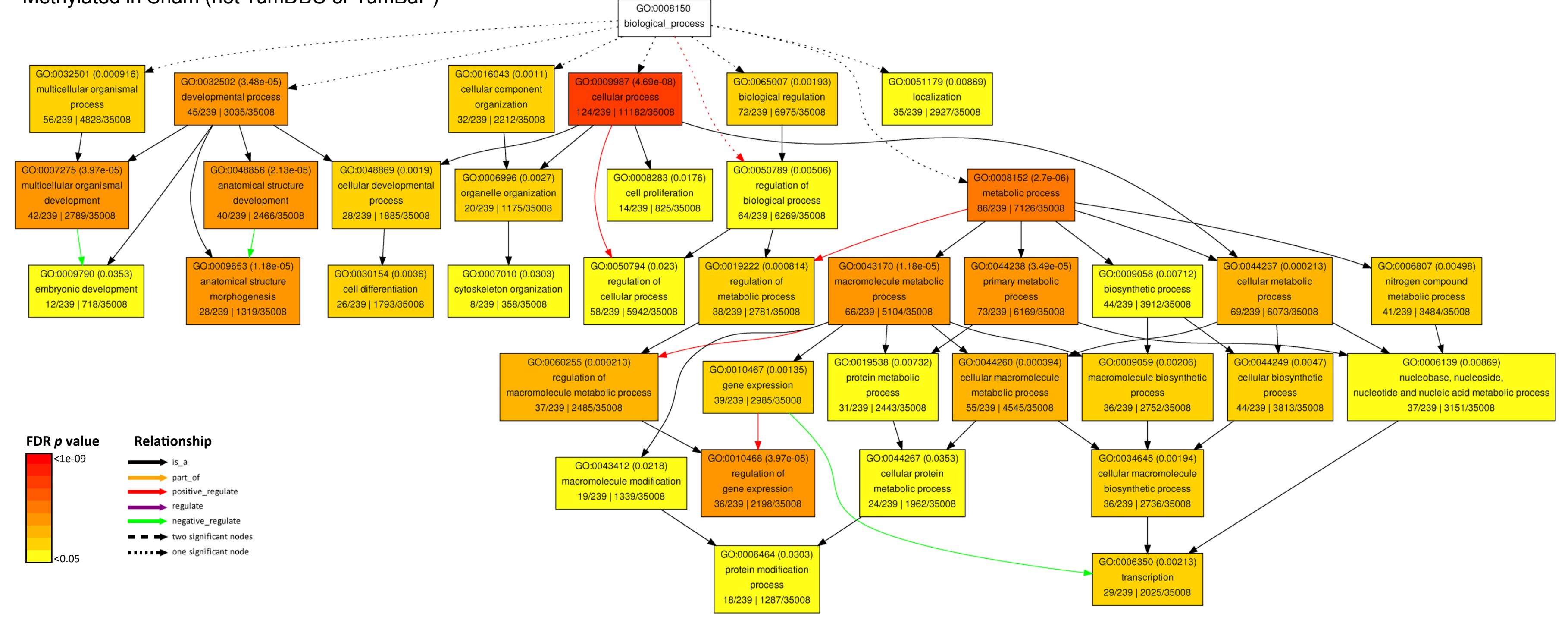

FIG. 14. Biological processes associated with genes differentially methylated in normal lung tissue from sham-initiated offspring. Gene Ontology map for GO terms representing hypermethylated gene promoters unique to sham tissues (not methylated in

lung tumors from DBC- or BaP-initiated offspring). Gene ontology analysis was

performed using the AgriGO singular enrichment analysis (SEA) tool

(http://bioinfo.cau.edu.cn/agriGO) against the mouse gene ontology database (Mouse

Genome Informatics). Significantly enriched GO terms (Generic GO Slim) were

determined using the Fisher test with an FDR-corrected significance level of $P<0.05$

(Yekutieli multi-test adjustment method) and the minimum number of mapping entries set at 5 genes. 


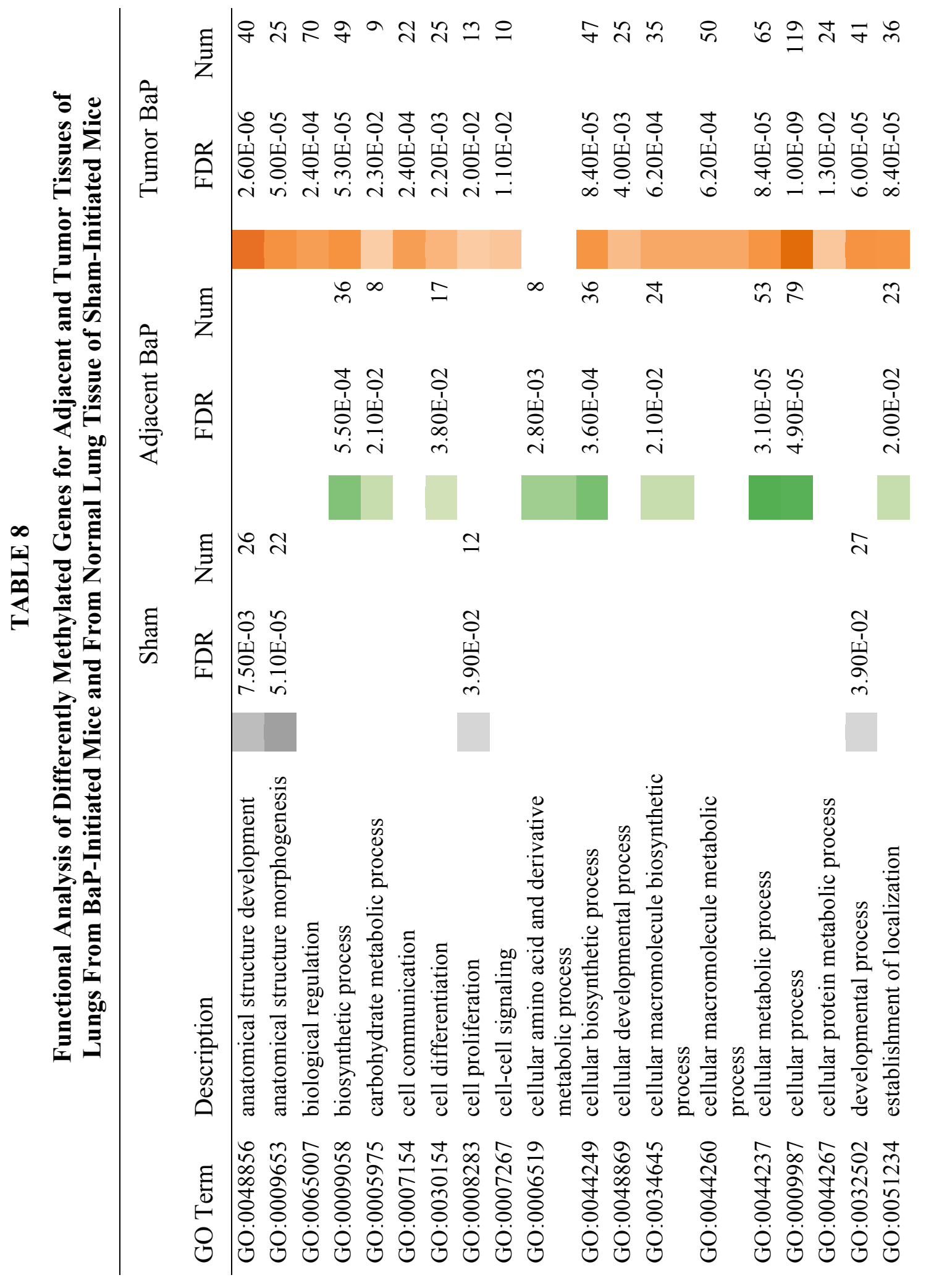




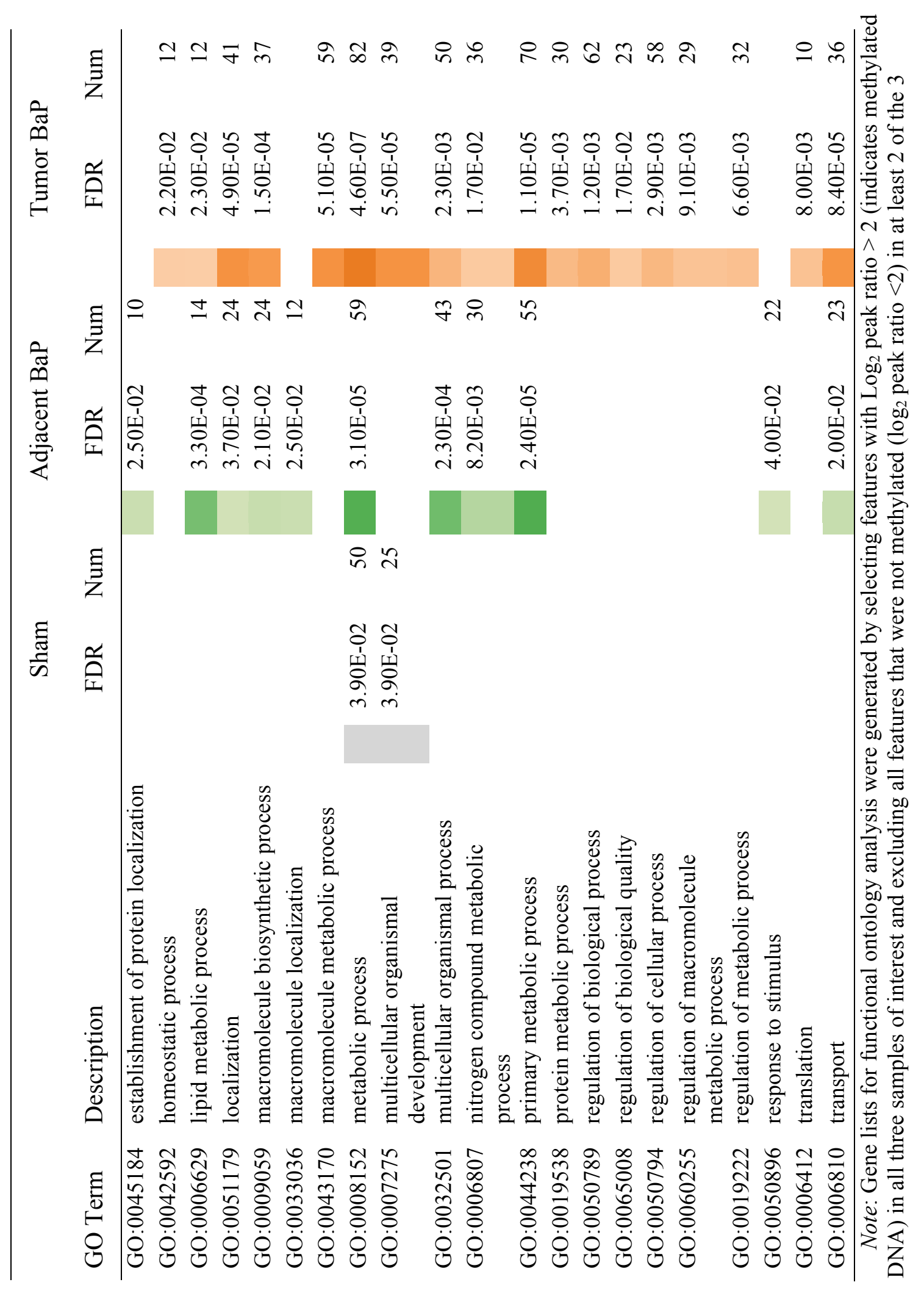




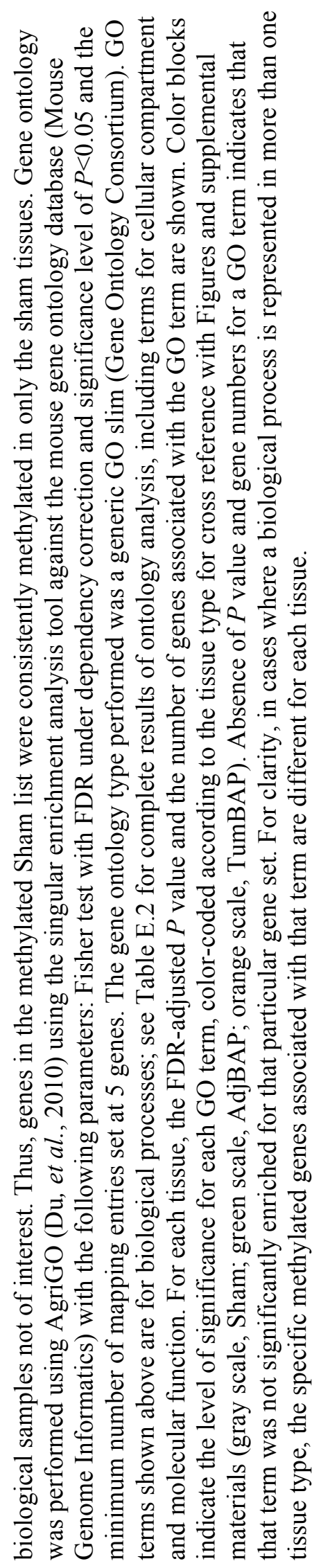


8.0E-03) GO terms, among other processes unique to the list. Both normal lung tissue of sham-initiated mice and tumor tissue of lungs from BaP-initiated mice yielded methylation profiles enriched for genes associated with the cell proliferation GO term $(\mathrm{FDR}=3.90 \mathrm{E}-02,2.0 \mathrm{E}-02$, respectively $)$

Fig. 15 and Table 9 depict functional analysis of differently methylated genes for tumor tissues obtained from lungs of DBC- and/or BaP-initiated mice and from normal lung tissue of sham-initiated mice. The enriched GO terms represented by all four comparison groups methylated gene lists total 42 , with normal lung tissue from shaminitiated mice containing the most functionally diverse list of genes. In addition to Table 5, Fig. 13-15 provide graphical representations of the enriched GO terms for each of the different tissue types compared. Normal lung tissue of sham-initiated mice yielded the most functionally diverse methylation profile enriched for genes in sham (not TumDBC or TumBaP) (Fig. 14) associated with embryonic development $(\mathrm{FDR}=3.50 \mathrm{E}-02)$, cell proliferation $(\mathrm{FDR}=1.80 \mathrm{E}-02)$, cell differentiation $(\mathrm{FDR}=3.60 \mathrm{E}-03)$, gene expression $(\mathrm{FDR}=1.30 \mathrm{E}-03)$ and transcription $(\mathrm{FDR}=2.10 \mathrm{E}-03)$ among the total 37 biological processes represented in the list. The least functionally diverse or most uniform gene list within this comparison was of tumor tissue from DBC-initiated mice. The TumDBC (not sham or TumBaP) methylation gene list was associated with only five enriched pathways (Fig. 15). The functional diversity between the lists of tumor $\mathrm{BaP}$ and tumor $\mathrm{DBC}$ plus $\mathrm{BaP}$ does not seem to differ much when based on the number of enriched pathways represented by each list, 20 and 22, respectively. However, there is diversity between the two lists as only ten of the 22 enriched GO terms for tumor DBC plus BaP are similarly 
A Methylated in TumDBC

(not sham or TumBaP)

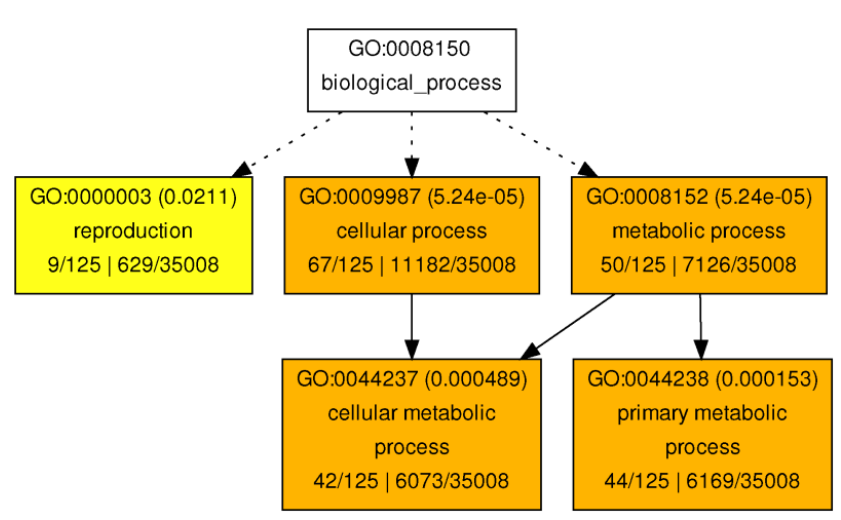

FDR $p$ value Relationship

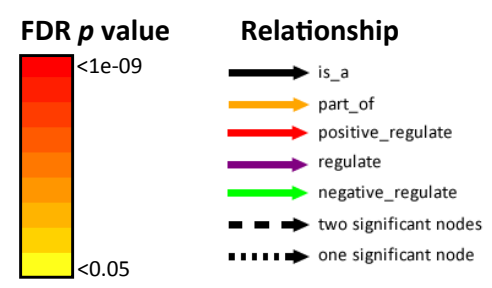

B

Methylated in TumBaP (not sham or TumDBC)

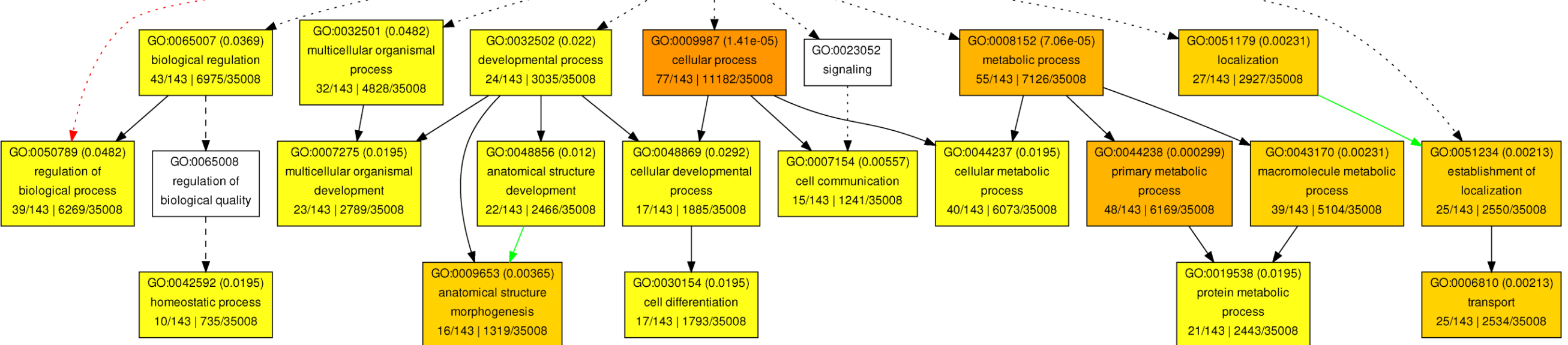

FIG. 15. Biological processes associated with genes differentially methylated in lung tumor for either DBC- or BaP initiated offspring. (A) GO map for GO terms representing hypermethylated gene promoters unique to tumor tissues from DBC-initiated mice (not methylated in sham lung tissue or lung tumors from BaP-initiated offspring). (B) $\mathrm{GO}$ map for $\mathrm{GO}$ terms representing hypermethylated gene promoters unique to tumor tissues from BaP-initiated mice (not methylated in sham lung tissue or lung tumors from DBC-

initiated offspring). Gene ontology analysis was performed using the AgriGO singular enrichment analysis (SEA) tool (http://bioinfo.cau.edu.cn/agriGO) against the mouse gene ontology database (Mouse Genome Informatics). Significantly enriched GO terms (Generic GO Slim) were determined using the Fisher test with an FDR-corrected

significance level of $P<0.05$ (Yekutieli multi-test adjustment method) and the minimum number of mapping entries set at 5 genes. 


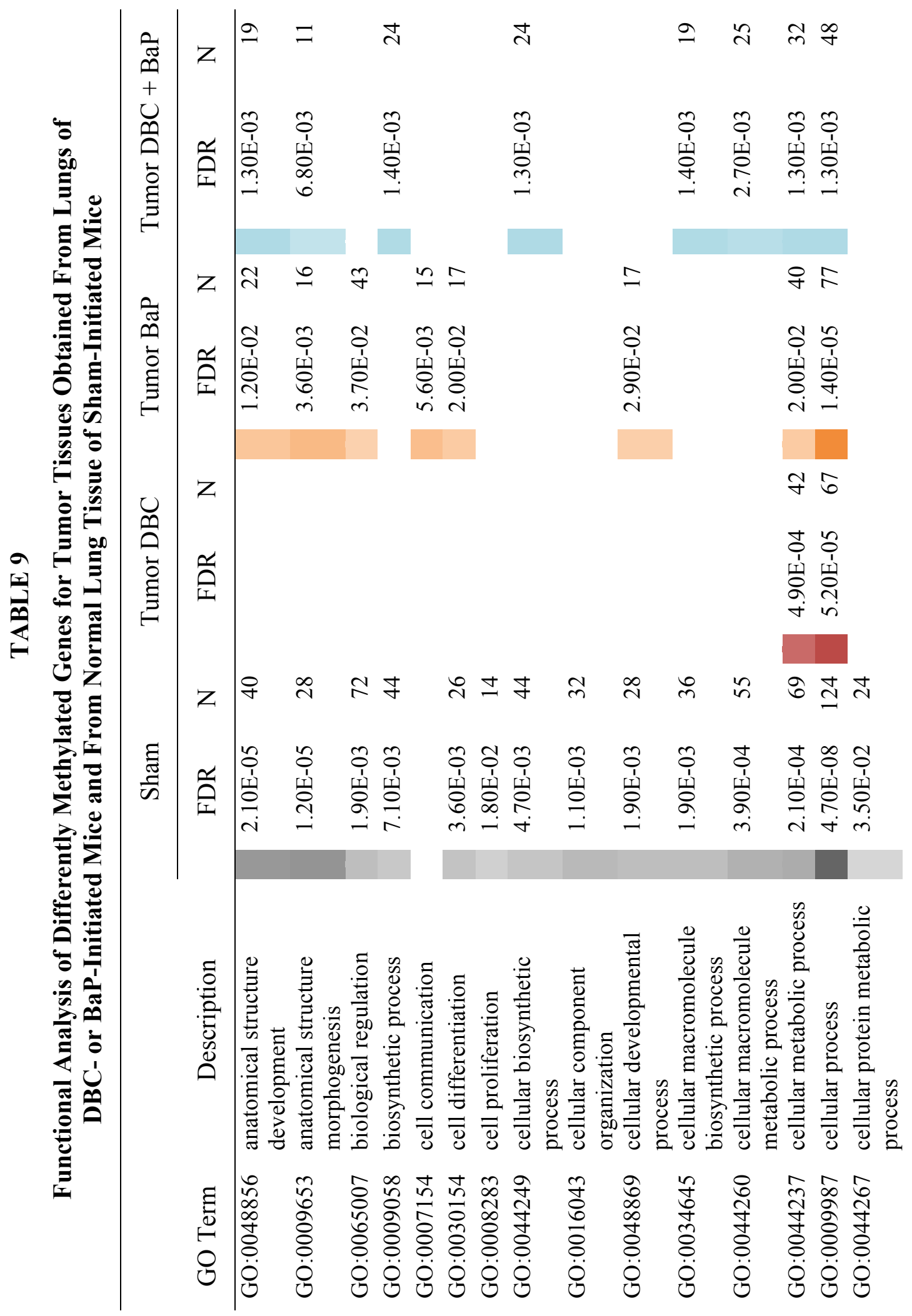




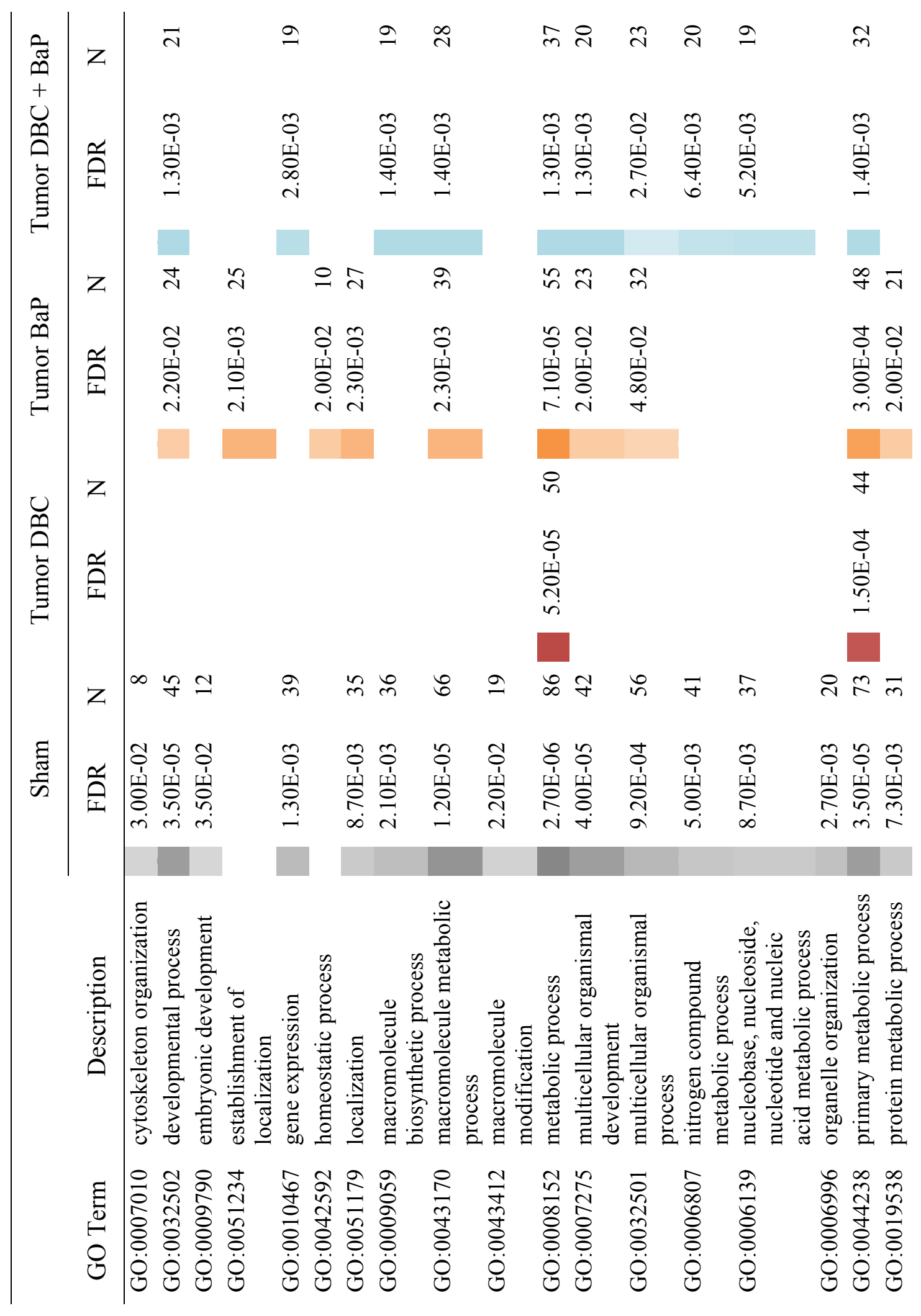




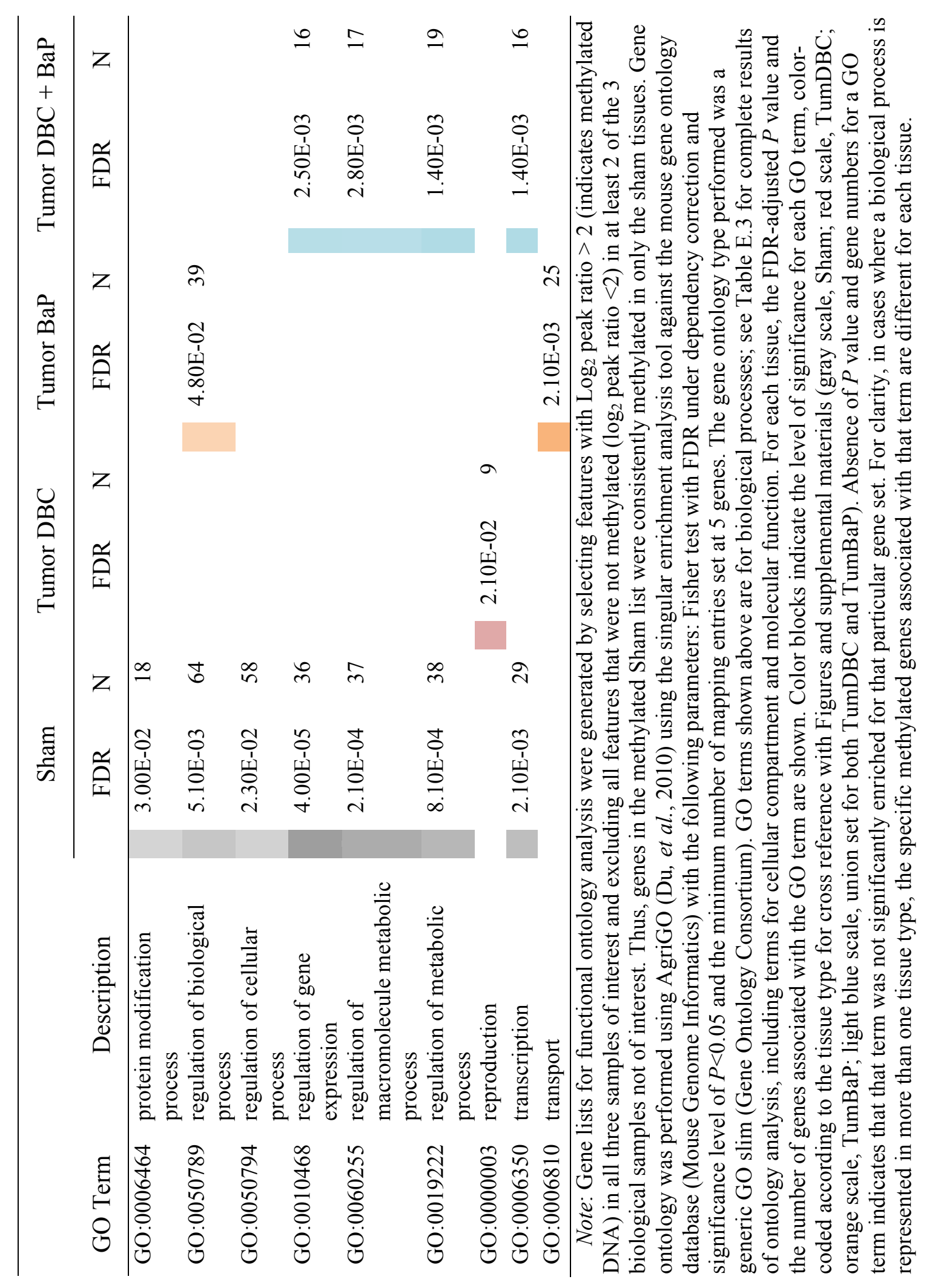


enriched within the tumor BaP gene list. Again, it is important to note that genes that made up the comparison lists for performing the $\mathrm{GO}$ analysis were entirely unique. GO terms enriched by hypermethylated gene promoters for lung tumor tissues from DBCand BaP-initiated offspring (not sham) (Fig. 13) were nucleobase, nucleoside, nucleotide and nucleic acid metabolic process $(\mathrm{FDR}=5.20 \mathrm{E}-03)$, regulation of gene expression $(\mathrm{FDR}=2.50 \mathrm{E}-03)$, gene expression $(\mathrm{FDR}=2.80 \mathrm{E}-03)$ and transcription $(\mathrm{FDR}=1.40 \mathrm{E}-$ 03), among the twelve pathways not similarly enriched for the $\mathrm{BaP}$ gene list. Tumor tissue from BaP-initiated mice yielded a methylation profile enriched for genes in TumBaP (not sham or TumDBC) (Fig. 15) associated with cell differentiation (FDR = 2.0E-02), cell communication $(\mathrm{FDR}=5.60 \mathrm{E}-03)$, transport $(\mathrm{FDR}=2.10 \mathrm{E}-03)$ and homeostatic process $(\mathrm{FDR}=2.0 \mathrm{E}-02)$ among other biological processes. See Fig. F.1F.10 in Appendix F (Fish, 2015e) for GO maps of other comparisons among tissue types using gene lists provided in Appendix D (Fish, 2015d). In summary, this gene ontology analysis showed that transplacental PAH exposure altered biological pathways associated with the regulation and control of many critical processes involved in lung tumorigenesis.

Ten genes were selected for further post-hoc inspection based on observations from the DNA methylation array and their relevance to tumorigenesis, including $B c l 2 l 11$, Bmp1, Fgfrlop, Hoxb1, Pdcd4, Casp7, Il11, Pten, Maea, and Tpd52l1. For each of these genes, the peak score data were aligned with sequence information to inspect patterns of methylation across all sample types using SignalMap software. Interestingly, for some genes selected for post-hoc inspection, the NimbleGen-predicted CpG island did not overlap with the identified region(s) of high methylation according to the array data. In 
addition, EMBOSS Cpgplot (Li, et al., 2015) was used as an independent prediction tool to confirm $\mathrm{CpG}$ islands for the selected genes. The Cpgplot-predicted $\mathrm{CpG}$ islands coincided with the NimbleGen-predicted $\mathrm{CpG}$ island for each gene. Furthermore, for several genes the Cpgplot-prediction revealed additional smaller $\mathrm{CpG}$ islands located apart from the NimbleGen-predicted CpG island. However, this observed discrepancy does not necessarily extend to all genes identified as differentially methylated using the NimbleGen platform, as additional random inspection showed good alignment (data not shown). The Bmpl gene promoter genomic DNA was clearly hypermethylated in sham lung tissues, as a large region spanning about $2400 \mathrm{bp}$ of methylation had peak scores above the 2.0 threshold (Fig. 16). This methylation pattern was consistent for all individuals in the sham group and was also very similar to the methylation pattern evident for normal adjacent lung tissues obtained from DBC- or BaP-initiated mice. Alternatively, methylation was largely absent in this region in tumor tissues obtained from DBC- or BaP-exposed offspring, save for two small regions of hypermethylation in one animal in the TumBaP group (Fig. 16). Similarly, the gene promoter genomic DNA for Fgfrlop (Fig. 17) was methylated in all sham lung tissue and adjacent normal tissues obtained from DBC- or BaP-initiated mice. Yet no tumor tissue samples had methylation surpassing the threshold score $\left(a-\log _{10} p\right.$-value $\left.>2\right)$. A related pattern was observed for the Hoxb1 promoter, for which all individuals in the sham group were similar to the methylation pattern evident for normal adjacent lung tissues (Fig. 18). Interestingly, within the tiled region of Hoxbl, there were two regions of methylation separated by 
FIG. 16. Methylation of Bmpl promoter in normal and tumor lung tissues. This figure illustrates methylation profiles for the promoter region of Bmpl obtained using the NimbleGen Mouse DNA Methylation 3×720K CpG Island Plus RefSeq Promoter Array. Chromosomal location, predicted $\mathrm{CpG}$ islands (black) and tiled regions are all mapped according to the NCBI37/mm9 mouse genome assembly. Also, $\mathrm{CpG}$ islands predicted by EMBOSS Cpgplot are shown in purple. Tracks representing control samples are shown in grey, adjacent normal DBC samples as blue, tumor DBC samples as red, adjacent normal $\mathrm{BaP}$ as green and tracks for tumor $\mathrm{BaP}$ as orange. Each sample is represented by two tracks, the peak scores and threshold score $>2.0$. The threshold score $>2.0$ track visualizes, by means of the solid bar, the region of peak scores that have surpassed the significance threshold and are thus considered to be hypermethylated. The peak score track displays the resulting score for each probe calculated as - $\log _{10} p$-value using the one-sided Kolmogorov-Smirnov test. 
Bmp1 [14:70,874,364-70,920,067:-1]

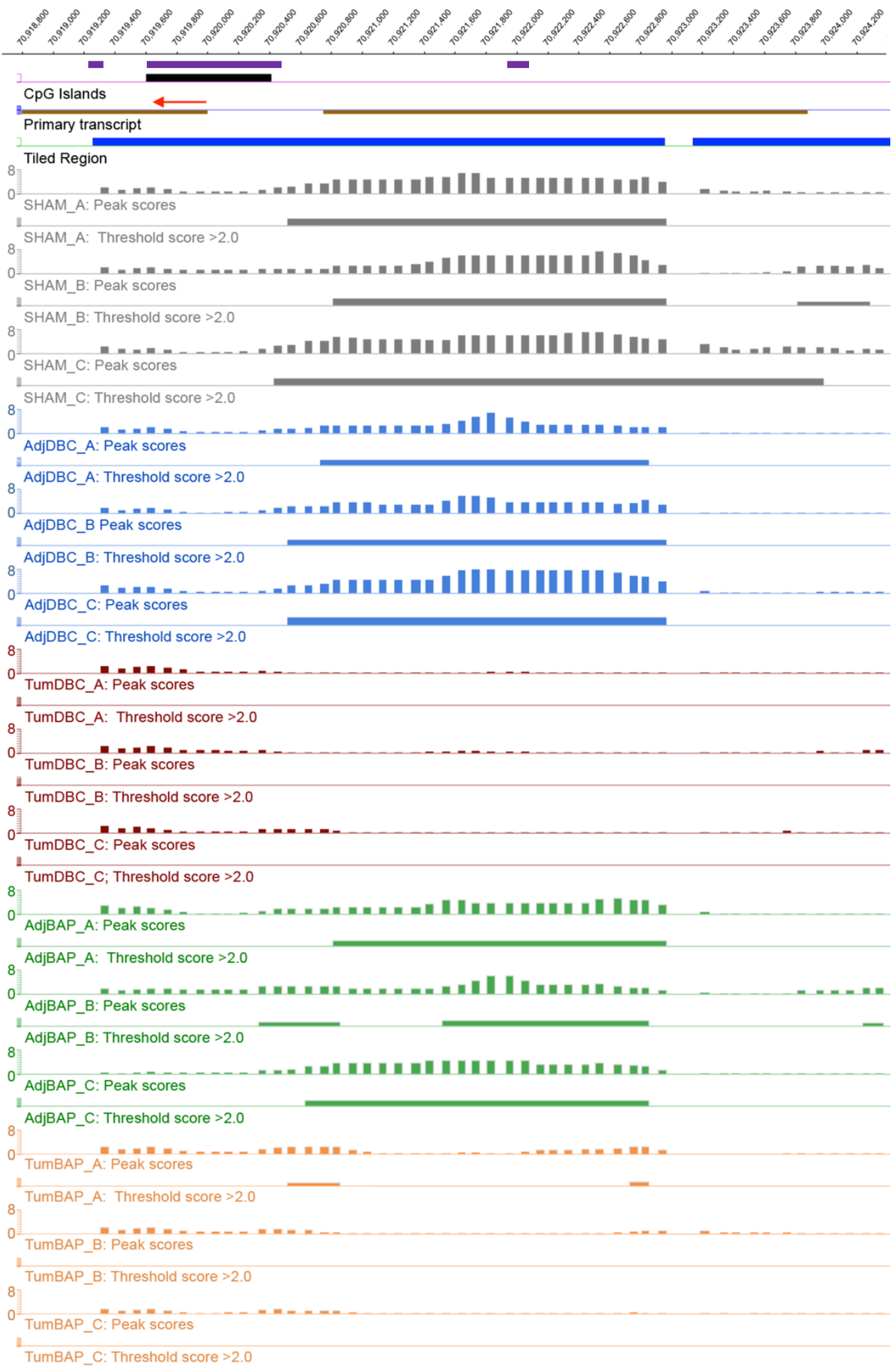


FIG. 17. Methylation of Fgfrlop promoter in normal and tumor lung tissues. This figure illustrates methylation profiles for the promoter region of Fgfr lop obtained using the NimbleGen Mouse DNA Methylation $3 \times 720 \mathrm{~K}$ CpG Island Plus RefSeq Promoter Array. Chromosomal location, predicted $\mathrm{CpG}$ islands (black) and tiled regions are all mapped according to the $\mathrm{NCBI} 37 / \mathrm{mm} 9$ mouse genome assembly. Also, $\mathrm{CpG}$ islands predicted by EMBOSS Cpgplot are shown in purple. Tracks representing control samples are shown in grey, adjacent normal DBC samples as blue, tumor DBC samples as red, adjacent normal $\mathrm{BaP}$ as green and tracks for tumor $\mathrm{BaP}$ as orange. Each sample is represented by two tracks, the peak scores and threshold score $>2.0$. The threshold score $>2.0$ track visualizes, by means of the solid bar, the region of peak scores that have surpassed the significance threshold and are thus considered to be hypermethylated. The peak score track displays the resulting score for each probe calculated as $-\log _{10} \mathrm{p}$-value using the one-sided Kolmogorov-Smirnov test. 
Fgfr1op [17: 8,358,366-8,389,669:1]

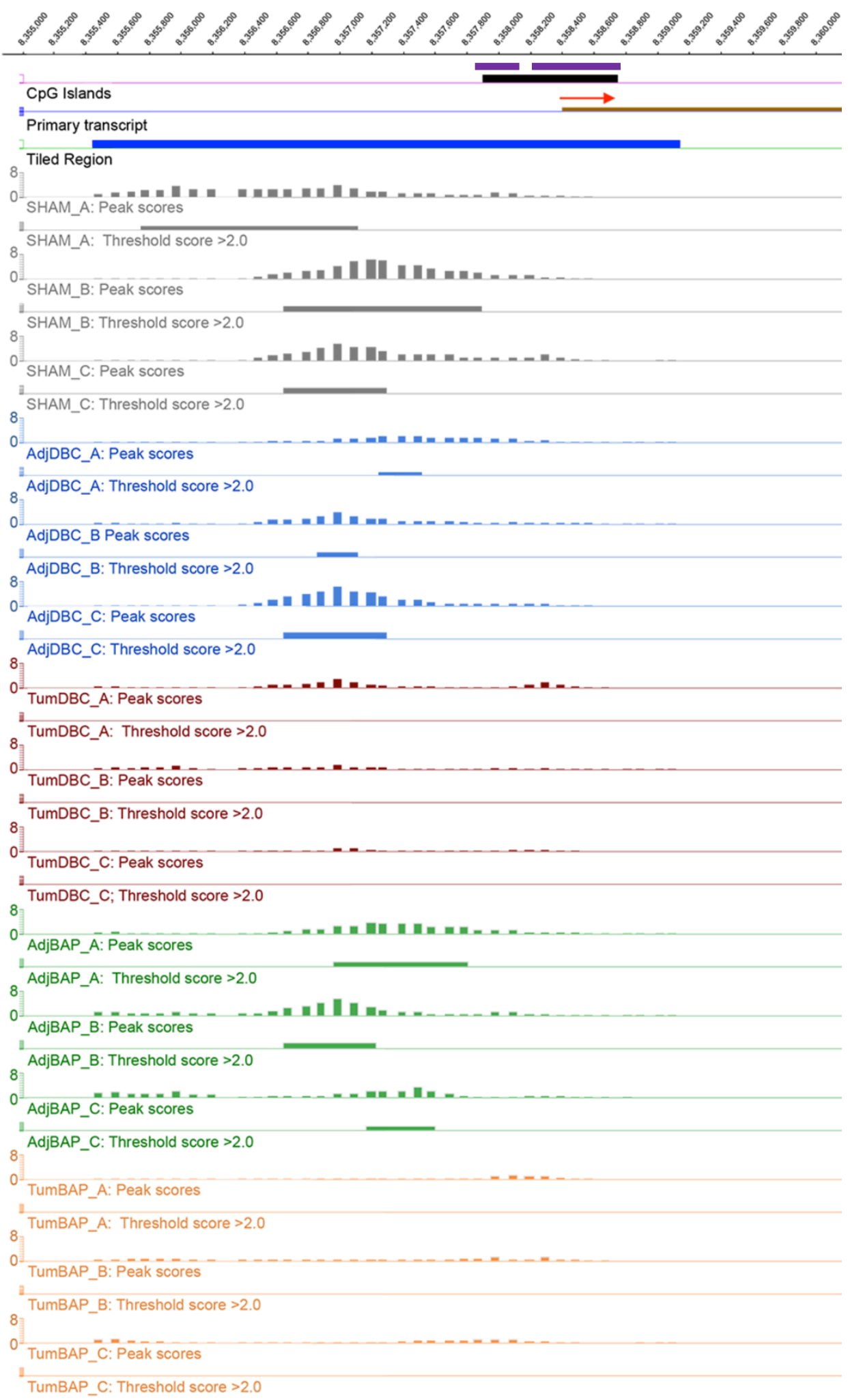


FIG. 18. Methylation of Hoxb1 promoter in normal and tumor lung tissues. This figure illustrates methylation profiles for the promoter region of Hoxbl obtained using the NimbleGen Mouse DNA Methylation $3 \times 720 \mathrm{~K}$ CpG Island Plus RefSeq Promoter Array. Chromosomal location, predicted $\mathrm{CpG}$ islands (black) and tiled regions are all mapped according to the NCBI37/mm9 mouse genome assembly. Also, CpG islands predicted by EMBOSS Cpgplot are shown in purple. Tracks representing control samples are shown in grey, adjacent normal DBC samples as blue, tumor DBC samples as red, adjacent normal $\mathrm{BaP}$ as green and tracks for tumor $\mathrm{BaP}$ as orange. Each sample is represented by two tracks, the peak scores and threshold score $>2.0$. The threshold score $>2.0$ track visualizes, by means of the solid bar, the region of peak scores that have surpassed the significance threshold and are thus considered to be hypermethylated. The peak score track displays the resulting score for each probe calculated as $-\log _{10} \mathrm{p}$-value using the one-sided Kolmogorov-Smirnov test. 
Hoxb1 [11:96227066-96229570:1]

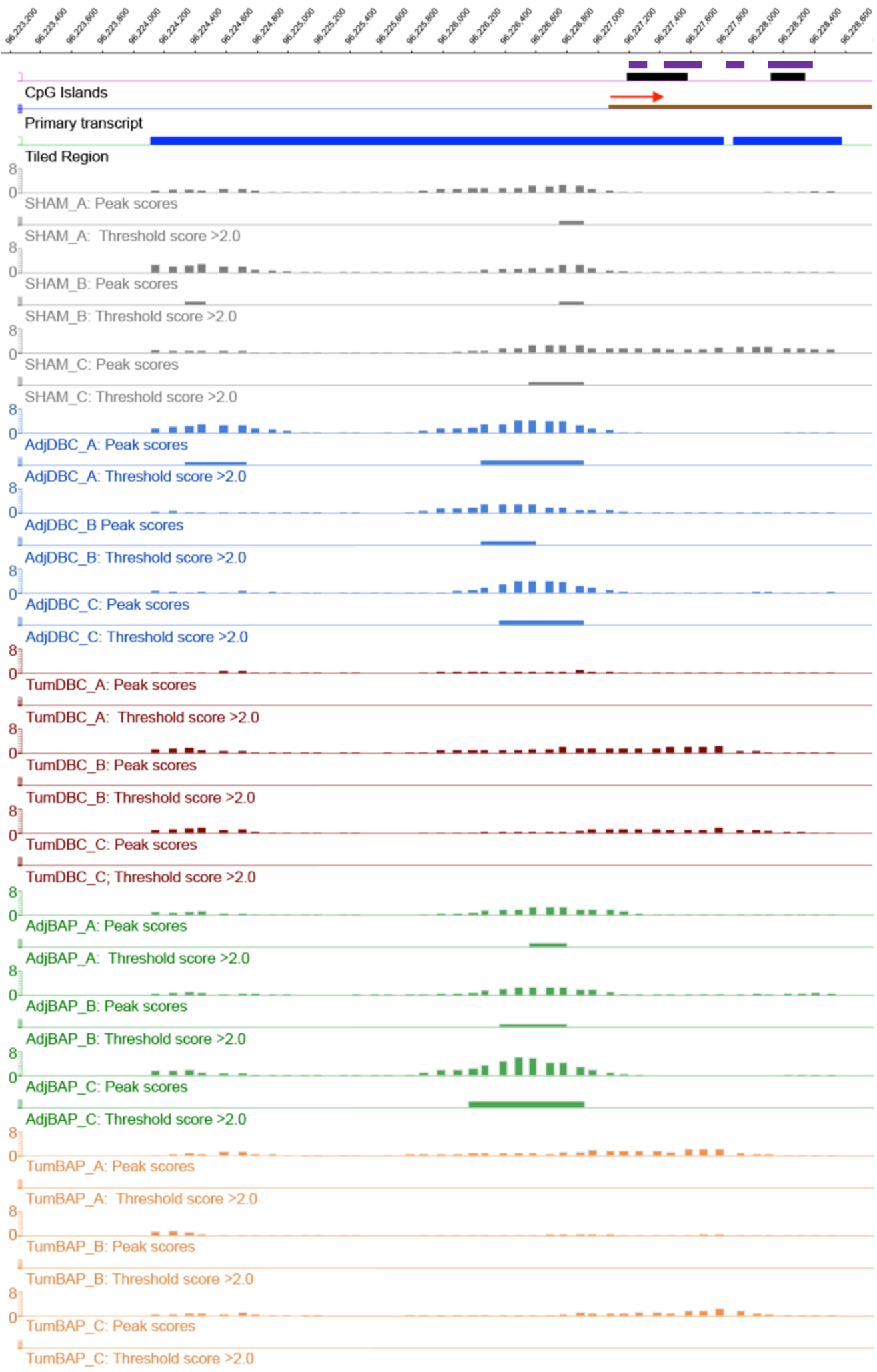


about $1,600 \mathrm{bps}$, for both the sham group and the adjacent normal tissues obtained from DBC-initiated mice.

In contrast, the methylation pattern across sample types was entirely different for $B c l 2 l 11$, which was highly methylated in all tumor samples and had relatively no methylation of adjacent normal or sham samples (Fig. 19). The methylated regions for Bcl2l11 ranged from $500 \mathrm{bp}$ to $1500 \mathrm{bp}$, and all but one $\mathrm{BaP}$ tumor sample were hypermethylated within the same 1700 bp span (location Chr2: 127949400-127951100). A similar pattern was observed for the $\mathrm{Pdcd} 4$ promoter, for which hypermethylation was evident for a short region (approximately $400 \mathrm{bp}$ ) in TumDBC tissue and a region of varying length (400 to $1200 \mathrm{bp}$ ) in TumBaP tissues (Fig 20). The $P d c d 4$ gene promoter was mostly not methylated in normal adjacent lung tissues and sham tissues. A related methylation pattern for Pten was observed, for which methylation peaks were evident within all the DBC-initiated tumor samples (Fig. 21). A major difference was observed in that methylation was largely absent from the BaP-exposed offspring. The Pten gene promoter was mostly not methylated in normal adjacent lung tissues and sham tissues.

For both Casp7 (Fig 22) and IL11 (Fig 23) gene promoters, the methylation pattern was consistent for all the sham tissue samples with methylation peak scores above the 2.0 threshold for regions of about 200 to $600 \mathrm{bp}$ in length. While some methylation was apparent in other tissue types, none of the peak score values for those samples exceeded the threshold indicating hypermethylation. 
FIG. 19. Methylation of $B c l 2 l 11$ promoter in normal and tumor lung tissues. This figure illustrates methylation profiles for the promoter region of $B c l 2 l 11$ obtained using the NimbleGen Mouse DNA Methylation $3 \times 720 \mathrm{~K}$ CpG Island Plus RefSeq Promoter Array. Chromosomal location, predicted $\mathrm{CpG}$ islands (black) and tiled regions are all mapped according to the NCBI37/mm9 mouse genome assembly. Also, $\mathrm{CpG}$ islands predicted by EMBOSS Cpgplot are shown in purple. Tracks representing control samples are shown in grey, adjacent normal DBC samples as blue, tumor DBC samples as red, adjacent normal $\mathrm{BaP}$ as green and tracks for tumor $\mathrm{BaP}$ as orange. Each sample is represented by two tracks, the peak scores and threshold score $>2.0$. The threshold score $>2.0$ track visualizes, by means of the solid bar, the region of peak scores that have surpassed the significance threshold and are thus considered to be hypermethylated. The peak score track displays the resulting score for each probe calculated as $-\log _{10} \mathrm{p}$-value using the one-sided Kolmogorov-Smirnov test. 
Bc/2/11 [2:127951774-127988283:1]

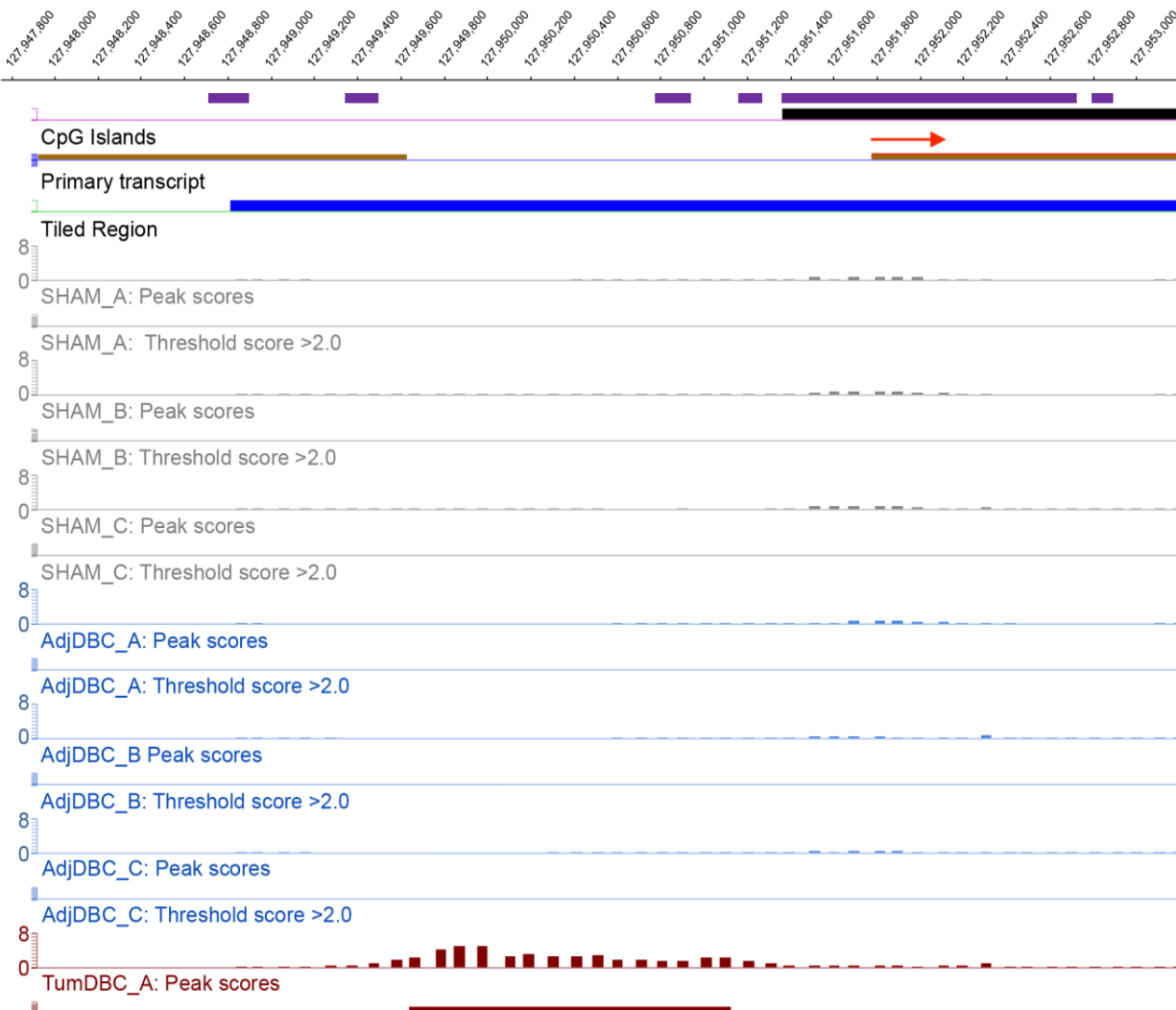

$8_{0}$ TumDBC_A: Threshold score $>2.0$

TumDBC_B: Peak scores

TumDBC_B: Threshold score $>2.0$

8

O TumDBC_C: Peak scores

"TumDBC_C; Threshold score $>2.0$

8

0

AdjBAP_A: Peak scores

AdjBAP_A: Threshold score $>2.0$

AdjBAP_B: Peak scores

AdjBAP_B: Threshold score $>2.0$

AdjBAP_C: Peak scores

AdjBAP_C: Threshold score $>2.0$

TumBAP_A: Peak scores

TumBAP_A: Threshold score $>2.0$

TumBAP_B: Peak scores

TumBAP_B: Threshold score $>2.0$

0 TumBAP C: Peak scores

TumBAP_C: Threshold score $>2.0$ 
FIG. 20. Methylation of $P d c d 4$ promoter in normal and tumor lung tissues. This figure illustrates methylation profiles for the promoter region of $P d c d 4$ obtained using the NimbleGen Mouse DNA Methylation 3×720K CpG Island Plus RefSeq Promoter Array. Chromosomal location, predicted $\mathrm{CpG}$ islands (black) and tiled regions are all mapped according to the NCBI37/mm9 mouse genome assembly. Also, $\mathrm{CpG}$ islands predicted by EMBOSS Cpgplot are shown in purple. Tracks representing control samples are shown in grey, adjacent normal DBC samples as blue, tumor DBC samples as red, adjacent normal $\mathrm{BaP}$ as green and tracks for tumor $\mathrm{BaP}$ as orange. Each sample is represented by two tracks, the peak scores and threshold score $>2.0$. The threshold score $>2.0$ track visualizes, by means of the solid bar, the region of peak scores that have surpassed the significance threshold and are thus considered to be hypermethylated. The peak score track displays the resulting score for each probe calculated as $-\log _{10} \mathrm{p}$-value using the one-sided Kolmogorov-Smirnov test. 


\section{Pdcd4 [19:53966721-54004351:1]}

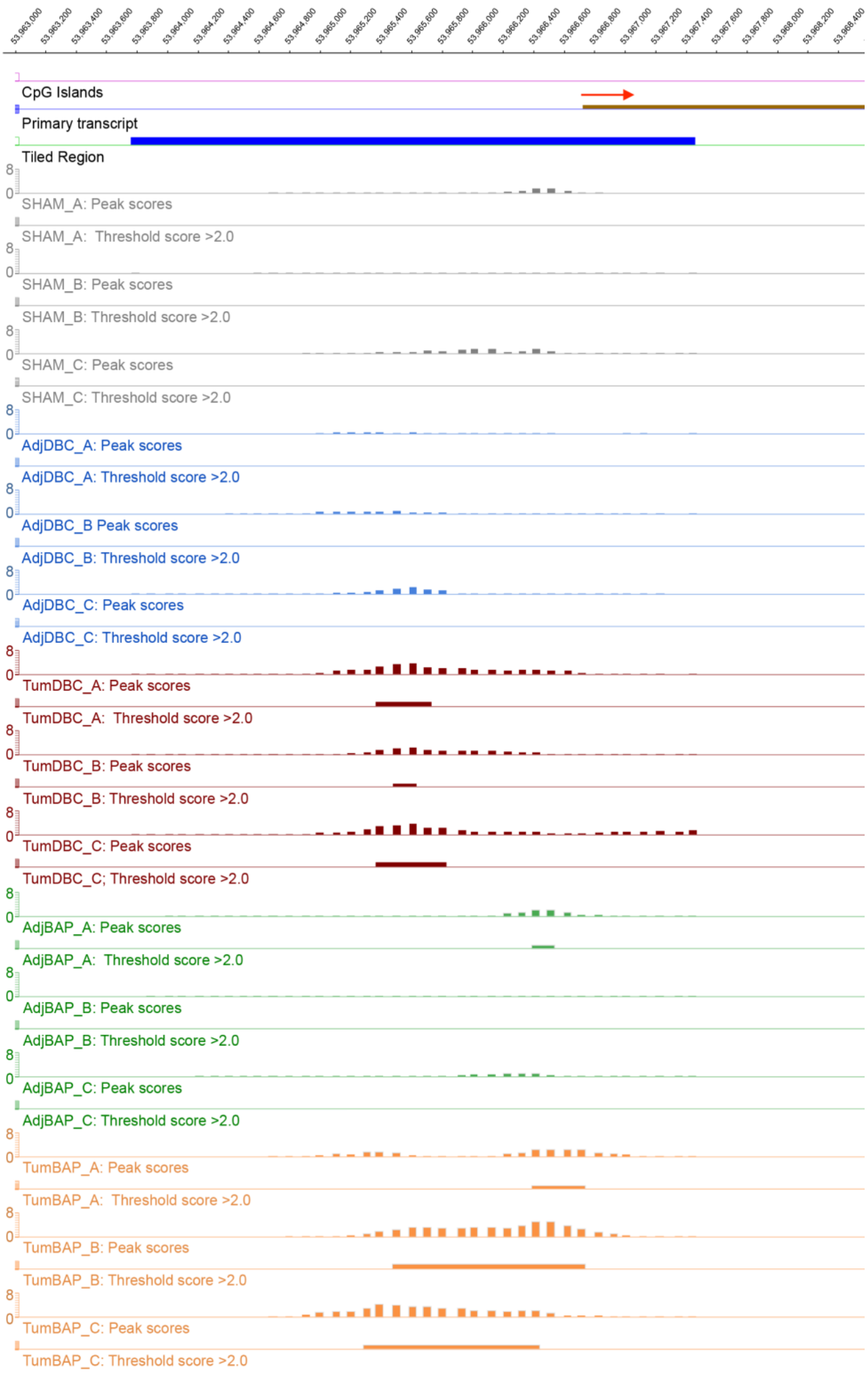


FIG. 21. Methylation of Pten promoter in normal and tumor lung tissues. This figure illustrates methylation profiles for the promoter region of Pten obtained using the NimbleGen Mouse DNA Methylation 3×720K CpG Island Plus RefSeq Promoter Array. Chromosomal location, predicted $\mathrm{CpG}$ islands (black) and tiled regions are all mapped according to the NCBI37/mm9 mouse genome assembly. Also, $\mathrm{CpG}$ islands predicted by EMBOSS Cpgplot are shown in purple. Tracks representing control samples are shown in grey, adjacent normal DBC samples as blue, tumor DBC samples as red, adjacent normal $\mathrm{BaP}$ as green and tracks for tumor $\mathrm{BaP}$ as orange. Each sample is represented by two tracks, the peak scores and threshold score $>2.0$. The threshold score $>2.0$ track visualizes, by means of the solid bar, the region of peak scores that have surpassed the significance threshold and are thus considered to be hypermethylated. The peak score track displays the resulting score for each probe calculated as $-\log _{10} \mathrm{p}$-value using the one-sided Kolmogorov-Smirnov test. 
Pten [19:32831987-32900650:1]

CpG Islands
Primary transcript

Tiled Region

8

SHAM_A: Peak scores

SHAM_A: Threshold score $>2.0$

${ }^{3}$

SHAM_B: Peak scores

SHAM_B: Threshold score $>2.0$

8

SHAM_C: Peak scores

SHAM_C: Threshold score $>2.0$

0

AdjDBC_A: Peak scores

AdjDBC_A: Threshold score $>2.0$

0

AdjDBC_B Peak scores

AdjDBC_B: Threshold score $>2.0$

AdjDBC_C: Peak scores

AdjDBC_C: Threshold score $>2.0$

$0^{8}$

TumDBC_A: Peak scores

TumDBC_A: Threshold score $>2.0$

$8_{\text {寻 }}$

0 TumDBC B: Peak

TumDBC_B: Threshold score $>2.0$

8

0 TumDBC_C: Peak scores

TumDBC C; Threshold score $>20$

8

OdjBAP_A: Peak scores

AdjBAP_A: Threshold score $>2.0$

0

AdjBAP_B: Peak scores

AdjBAP_B: Threshold score $>2.0$

0 AdjBAP_C: Peak scores

AdjBAP_C: Threshold score $>2.0$

0 TumBAP_A: Peak scores

TumBAP A: Threshold score $>2.0$

TumBAP_B: Peak scores

TumBAP_B: Threshold score $>2.0$

TumBAP C: Peak scores

TumBAP_C: Threshold score $>2.0$ 
FIG. 22. Methylation of Casp 7 promoter in normal and tumor lung tissues. This figure illustrates methylation profiles for the promoter region of Casp 7 obtained using the NimbleGen Mouse DNA Methylation 3×720K CpG Island Plus RefSeq Promoter Array. Chromosomal location, predicted $\mathrm{CpG}$ islands (black) and tiled regions are all mapped according to the NCBI37/mm9 mouse genome assembly. Also, $\mathrm{CpG}$ islands predicted by EMBOSS Cpgplot are shown in purple. Tracks representing control samples are shown in grey, adjacent normal DBC samples as blue, tumor DBC samples as red, adjacent normal $\mathrm{BaP}$ as green and tracks for tumor $\mathrm{BaP}$ as orange. Each sample is represented by two tracks, the peak scores and threshold score $>2.0$. The threshold score $>2.0$ track visualizes, by means of the solid bar, the region of peak scores that have surpassed the significance threshold and are thus considered to be hypermethylated. The peak score track displays the resulting score for each probe calculated as $-\log _{10} \mathrm{p}$-value using the one-sided Kolmogorov-Smirnov test. 
Casp7 [19:56471619-56516834:1]

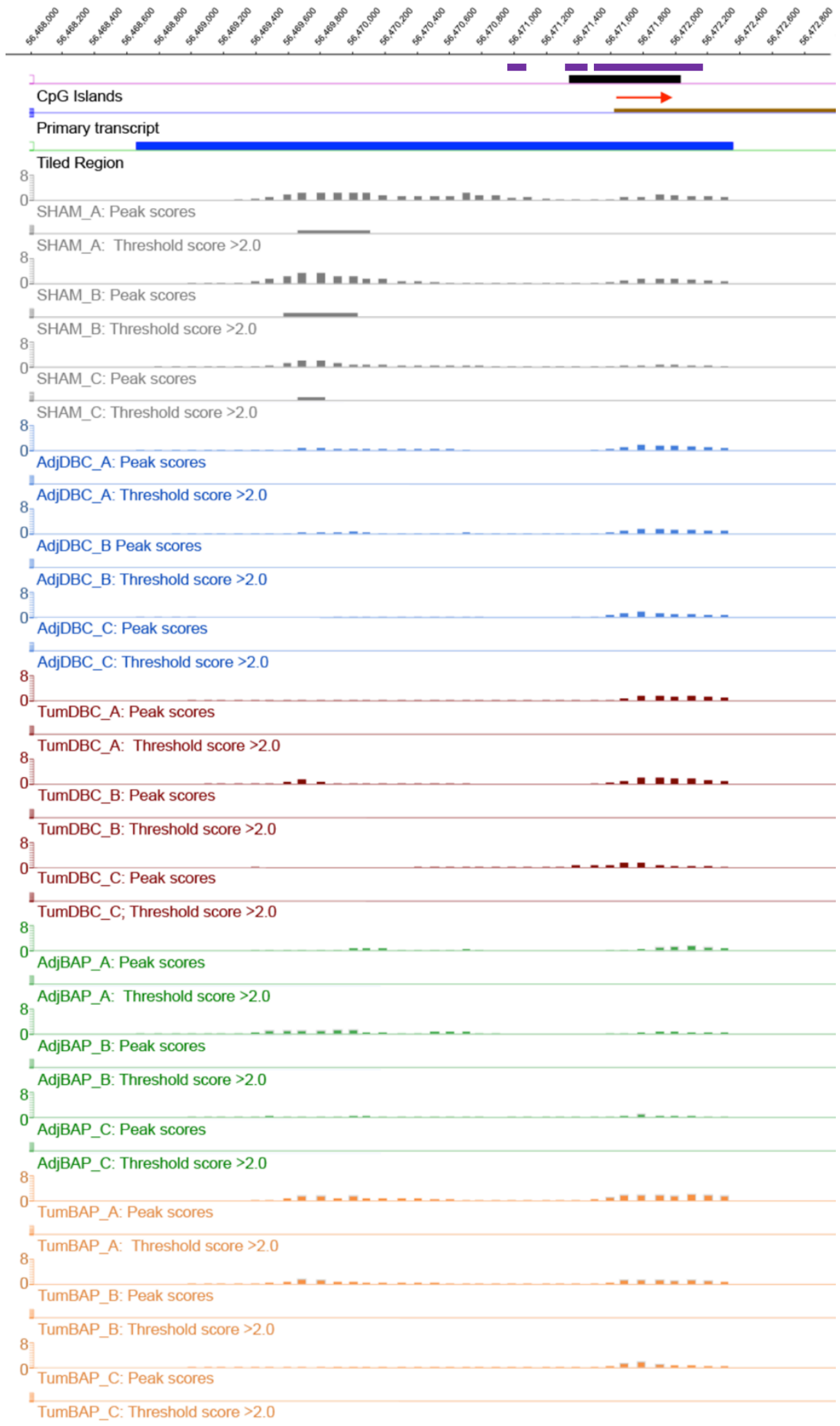


FIG. 23. Methylation of $I l 11$ promoter in normal and tumor lung tissues. This figure illustrates methylation profiles for the promoter region of $I l 11$ obtained using the NimbleGen Mouse DNA Methylation 3×720K CpG Island Plus RefSeq Promoter Array. Chromosomal location, predicted $\mathrm{CpG}$ islands (black) and tiled regions are all mapped according to the NCBI37/mm9 mouse genome assembly. Also, $\mathrm{CpG}$ islands predicted by EMBOSS Cpgplot are shown in purple. Tracks representing control samples are shown in grey, adjacent normal DBC samples as blue, tumor DBC samples as red, adjacent normal $\mathrm{BaP}$ as green and tracks for tumor $\mathrm{BaP}$ as orange. Each sample is represented by two tracks, the peak scores and threshold score $>2.0$. The threshold score $>2.0$ track visualizes, by means of the solid bar, the region of peak scores that have surpassed the significance threshold and are thus considered to be hypermethylated. The peak score track displays the resulting score for each probe calculated as $-\log _{10} \mathrm{p}$-value using the one-sided Kolmogorov-Smirnov test. 
73

II11 [7:4724657-4734459:-1]

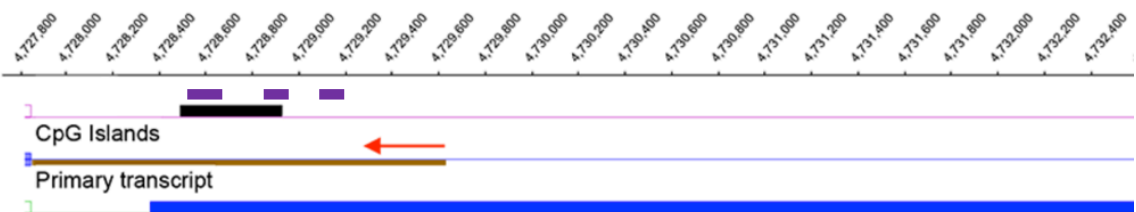

Tiled Region

SHAM_A: Peak scores

SHAM_A: Threshold score $>2.0$

SHAM_B: Peak scores

SHAM_B: Threshold score $>2.0$

0

SHAM_C: Peak scores

SHAM_C: Threshold score $>2.0$

AdjDBC_A: Peak scores

AdjDBC_A: Threshold score $>2.0$

AdjDBC_B Peak scores

AdjDBC_B: Threshold score $>2.0$

AdjDBC_C: Peak scores

AdjDBC_C: Threshold score $>2.0$

TumDBC_A: Peak scores

TumDBC_A: Threshold score $>2.0$

TumDBC_B: Peak scores

TumDBC_B: Threshold score $>2.0$

8

TumDBC_C: Peak scores

TumDBC_C; Threshold score $>2.0$

AdjBAP_A: Peak scores

AdjBAP_A: Threshold score $>2.0$

AdjBAP_B: Peak scores

AdjBAP_B: Threshold score $>2.0$

AdjBAP_C: Peak scores

AdjBAP_C: Threshold score $>2.0$

TumBAP_A: Peak scores

TumBAP_A: Threshold score $>2.0$

TumBAP_B: Peak scores

TumBAP_B: Threshold score $>2.0$

8

TumBAP_C: Peak scores

TumBAP_C: Threshold score $>2.0$ 
The promoter methylation pattern for the oncogene Maea was consistent in sham lung tissues across all individuals, as peak scores exceeded the 2.0 threshold for a region of approximately 1,000 bp (Fig 24). Smaller regions (200 to $400 \mathrm{bp}$ ) of hypermethylation were observed in one adjacent normal DBC sample, two adjacent normal BaP samples and one $\mathrm{BaP}$ tumor sample. All the methylated regions across all samples types for Maea range fell within the same 1,200 bp span (location Chr5: 33675200-33676400). Similarly, the Tpd52l1 gene promoter genomic DNA was methylated in all sham lung tissues, one normal adjacent $\mathrm{BaP}$ sample and two BaP tumor samples (Fig 25). Alternatively, methylation was mostly absent from the adjacent normal and DBC lung tumor tissue samples. In addition, all the methylated peaks of Tpd52ll's promoter were in the same area within the tiled region (location Chr10: 31166600-31167900).

Methylation patterns obtained from the Nimblgen genome-wide array were compared to profiles obtained by 454 amplicon sequencing for Cdkn2a, Dapk1, Mgmt and Rarb. According to the methylation profile obtained by the array, the $C d k n 2 a$ gene promoter was hypermethylated in one of three sham normal lung samples, two of three tumor samples and one of three in the normal adjacent tissues for both carcinogen initiated groups (Fig. 26). The gene promoter DNA for Dapkl was methylated across nearly all sample types, with methylated regions varying in length from $100 \mathrm{bp}$ to 1300 bp (Fig. 27). In contrast, the methylation pattern was markedly different for both $M g m t$ and Rarb. Some methylation was noted for the Mgmt promoter in two of the three sham normal tissues, but no significant methylation in any tissues from PAH-initiated animals (Fig. 28). Conversely, the Rarb gene promoter was devoid of any methylation in all 
FIG. 24. Methylation of Maea promoter in normal and tumor lung tissues. This figure illustrates methylation profiles for the promoter region of Maea obtained using the NimbleGen Mouse DNA Methylation 3×720K CpG Island Plus RefSeq Promoter Array. Chromosomal location, predicted $\mathrm{CpG}$ islands (black) and tiled regions are all mapped according to the NCBI37/mm9 mouse genome assembly. Also, $\mathrm{CpG}$ islands predicted by EMBOSS Cpgplot are shown in purple. Tracks representing control samples are shown in grey, adjacent normal DBC samples as blue, tumor DBC samples as red, adjacent normal $\mathrm{BaP}$ as green and tracks for tumor $\mathrm{BaP}$ as orange. Each sample is represented by two tracks, the peak scores and threshold score $>2.0$. The threshold score $>2.0$ track visualizes, by means of the solid bar, the region of peak scores that have surpassed the significance threshold and are thus considered to be hypermethylated. The peak score track displays the resulting score for each probe calculated as $-\log _{10} \mathrm{p}$-value using the one-sided Kolmogorov-Smirnov test. 
Maea [5:33678214-33715942:1]
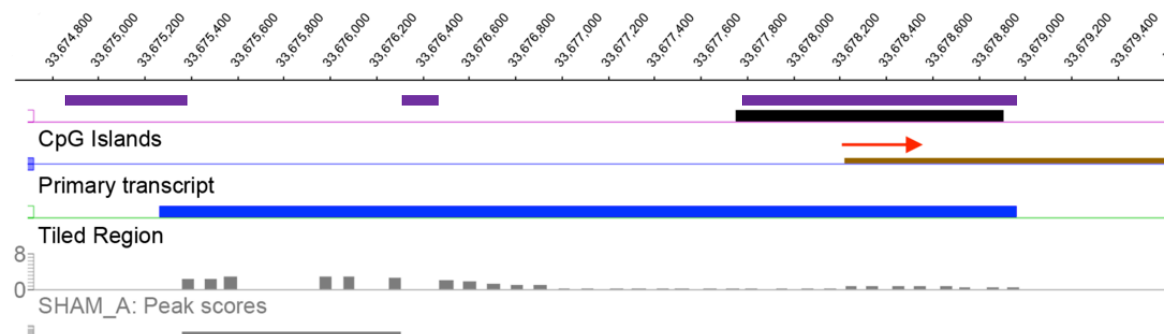

SHAM_A: Threshold score $>2.0$

8

SHAM_B: Peak scores

SHAM_B: Threshold score $>2.0$

8

0 SHAM_C: Peak scores

SHAM_C: Threshold score $>2.0$

0 1 and a

kcores

AdjDBC_A: Threshold score $>2.0$

0 AdjDBC_B Peak scores

AdjDBC_B: Threshold score $>2.0$

AdjDBC_C: Peak scores

AdjDBC_C: Threshold score $>2.0$

TumDBC_A: Peak scores

TumDBC_A: Threshold score $>2.0$

每

TumDBC_B: Peak scores

TumDBC_B: Threshold score $>2.0$

TumDBC_C: Peak scores

TumDBC C; Threshold score $>2.0$

8

OdjBAP_A: Peak scores

AdjBAP_A: Threshold score $>2.0$

AdjBAP_B: Peak scores

AdjBAP B: Threshold score $>2.0$

OdjBAP_C: Peak scores

AdjBAP_C: Threshold score $>2.0$

TumBAP_A: Peak scores

TumBAP A: Threshold score $>2.0$

TumBAP_B: Peak scores

TumBAP_B: Threshold score $>2.0$

TumBAP_C: Peak scores

TumBAP_C: Threshold score $>2.0$ 
FIG. 25. Methylation of Tpd52l1 promoter in normal and tumor lung tissues. This figure illustrates methylation profiles for the promoter region of Tpd52ll obtained using the NimbleGen Mouse DNA Methylation 3×720K CpG Island Plus RefSeq Promoter Array. Chromosomal location, predicted $\mathrm{CpG}$ islands (black) and tiled regions are all mapped according to the NCBI37/mm9 mouse genome assembly. Also, CpG islands predicted by EMBOSS Cpgplot are shown in purple. Tracks representing control samples are shown in grey, adjacent normal DBC samples as blue, tumor DBC samples as red, adjacent normal $\mathrm{BaP}$ as green and tracks for tumor $\mathrm{BaP}$ as orange. Each sample is represented by two tracks, the peak scores and threshold score $>2.0$. The threshold score $>2.0$ track visualizes, by means of the solid bar, the region of peak scores that have surpassed the significance threshold and are thus considered to be hypermethylated. The peak score track displays the resulting score for each probe calculated as $-\log _{10} \mathrm{p}$-value using the one-sided Kolmogorov-Smirnov test. 


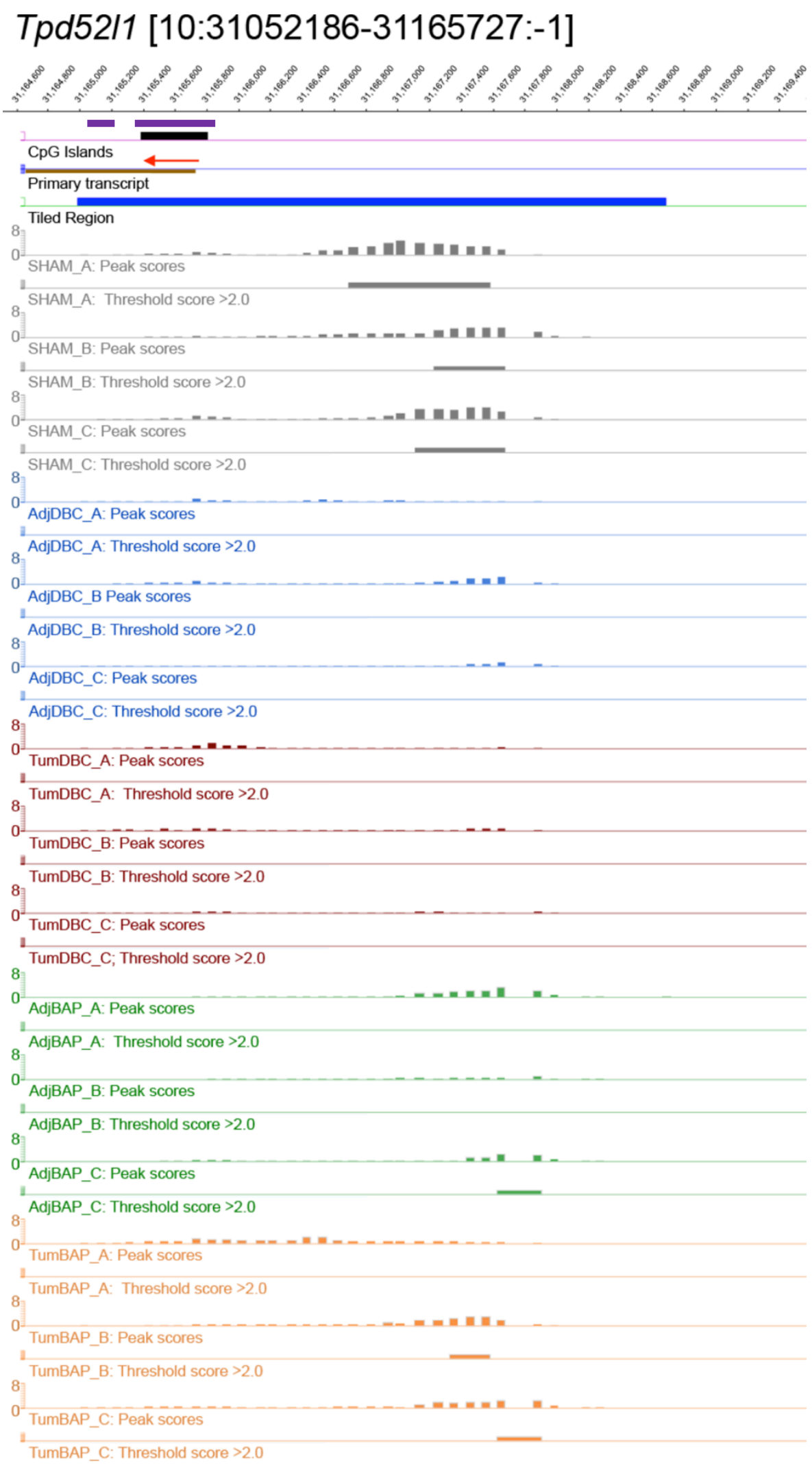


FIG. 26. Methylation of $C d k n 2 a$ promoter in normal and tumor lung tissues. This figure illustrates methylation profiles for the promoter region of $C d k n 2 a$ obtained using the NimbleGen Mouse DNA Methylation $3 \times 720 \mathrm{~K}$ CpG Island Plus RefSeq Promoter Array. Chromosomal location, predicted $\mathrm{CpG}$ islands (black) and tiled regions are all mapped according to the NCBI37/mm9 mouse genome assembly. Also, $\mathrm{CpG}$ islands predicted by EMBOSS Cpgplot are shown in purple. Tracks representing control samples are shown in grey, adjacent normal DBC samples as blue, tumor DBC samples as red, adjacent normal $\mathrm{BaP}$ as green and tracks for tumor $\mathrm{BaP}$ as orange. Bright pink indicates the region of this gene analyzed by bisulfite amplicon sequencing by 454 (data shown in Fig. 8). Each sample is represented by two tracks, the peak scores and threshold score $>2.0$. The threshold score $>2.0$ track visualizes, by means of the solid bar, the region of peak scores that have surpassed the significance threshold and are thus considered to be hypermethylated. The peak score track displays the resulting score for each probe calculated as $-\log _{10}$ p-value using the one-sided Kolmogorov-Smirnov test. 
Cdkn2a [4:88920377-88940557:-1]

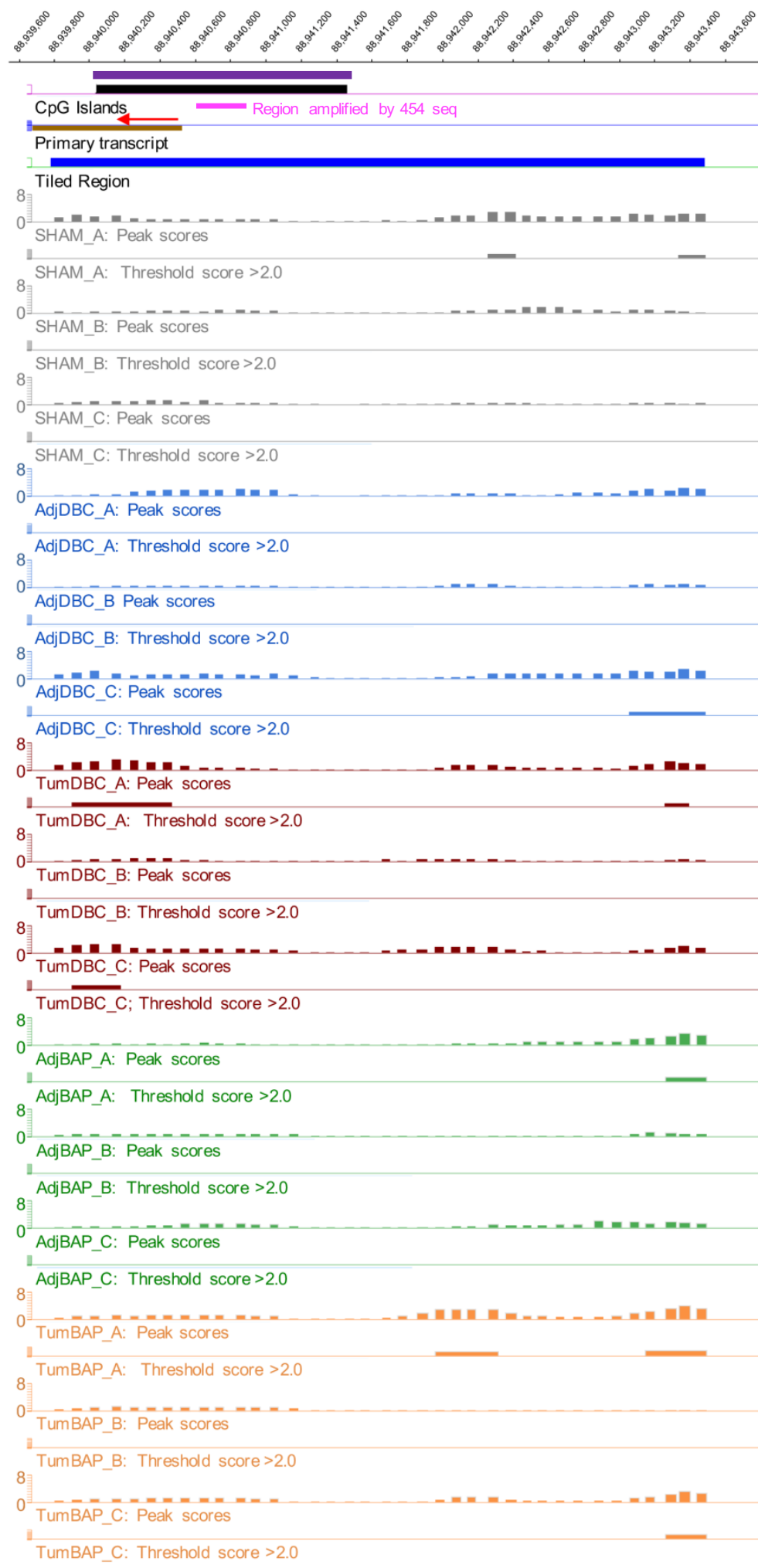


FIG. 27. Methylation of Dapkl promoter in normal and tumor lung tissues. This figure illustrates methylation profiles for the promoter region of Dapkl obtained using the NimbleGen Mouse DNA Methylation 3×720K CpG Island Plus RefSeq Promoter Array. Chromosomal location, predicted $\mathrm{CpG}$ islands and tiled regions are all mapped according to the NCBI37/mm9 mouse genome assembly. Also, $\mathrm{CpG}$ islands predicted by EMBOSS Cpgplot are shown in purple. Bright pink indicates the region of this gene analyzed by bisulfite amplicon sequencing by 454 (data shown in Fig. 9). Tracks representing control samples are shown in grey, adjacent normal DBC samples as blue, tumor $\mathrm{DBC}$ samples as red, adjacent normal $\mathrm{BaP}$ as green and tracks for tumor $\mathrm{BaP}$ as orange. Each sample is represented by two tracks, the peak scores and threshold score $>2.0$. The threshold score $>2.0$ track visualizes, by means of the solid bar, the region of peak scores that have surpassed the significance threshold and are thus considered to be hypermethylated. The peak score track displays the resulting score for each probe calculated as $-\log _{10}$ p-value using the one-sided Kolmogorov-Smirnov test. 
Dapk1 [13:60703308-60864546:1]

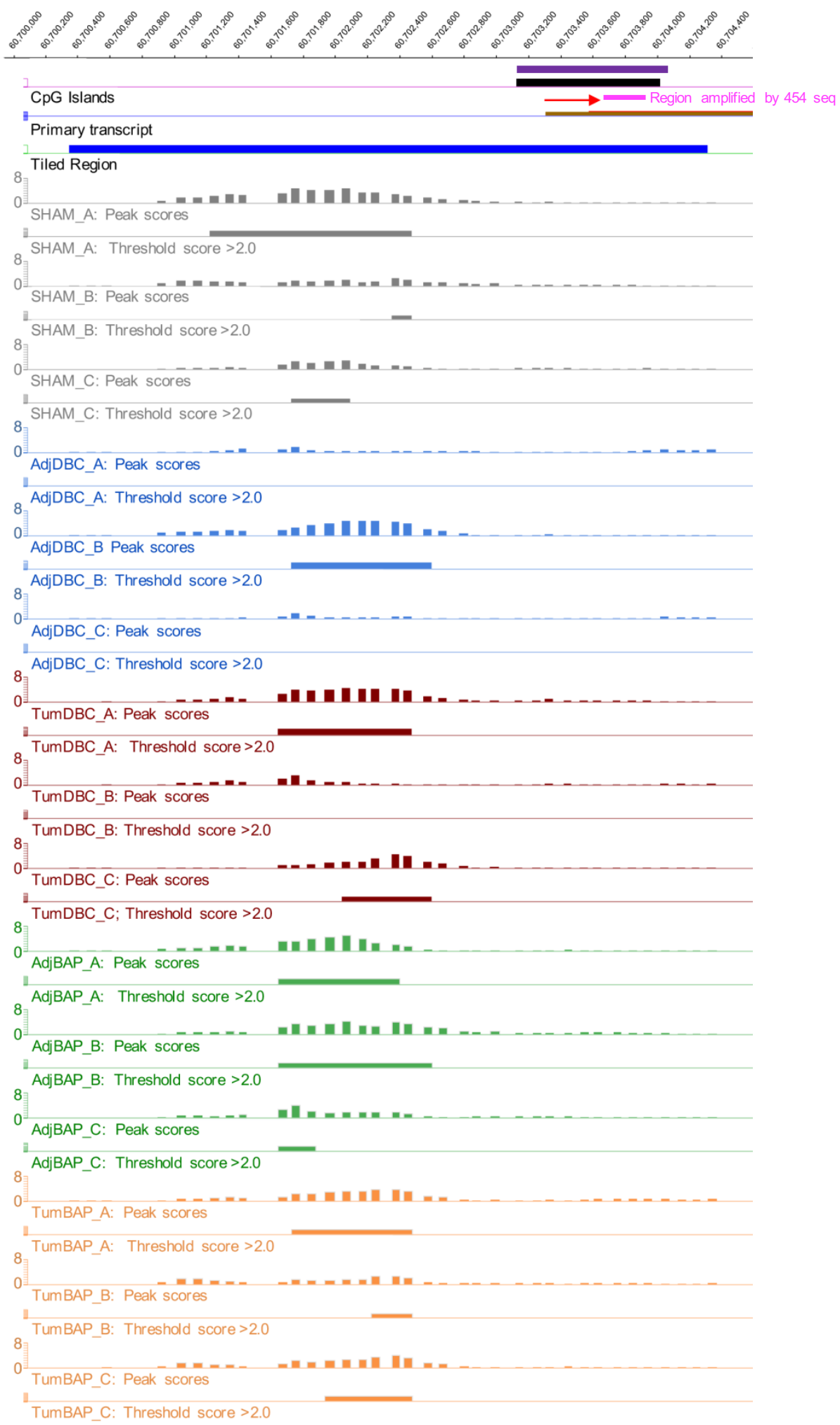


FIG. 28. Methylation of Mgmt promoter in normal and tumor lung tissues. This figure illustrates methylation profiles for the promoter region of $\mathrm{Mgmt}$ obtained using the NimbleGen Mouse DNA Methylation 3×720K CpG Island Plus RefSeq Promoter Array. Chromosomal location, predicted $\mathrm{CpG}$ islands and tiled regions are all mapped according to the NCBI37/mm9 mouse genome assembly. Also, $\mathrm{CpG}$ islands predicted by EMBOSS Cpgplot are shown in purple. Bright pink indicates the region of this gene analyzed by bisulfite amplicon sequencing by 454 (data shown in Fig. 10). Tracks representing control samples are shown in grey, adjacent normal DBC samples as blue, tumor DBC samples as red, adjacent normal $\mathrm{BaP}$ as green and tracks for tumor $\mathrm{BaP}$ as orange. Each sample is represented by two tracks, the peak scores and threshold score $>2.0$. The threshold score $>2.0$ track visualizes, by means of the solid bar, the region of peak scores that have surpassed the significance threshold and are thus considered to be hypermethylated. The peak score track displays the resulting score for each probe calculated as $-\log _{10}$ p-value using the one-sided Kolmogorov-Smirnov test. 


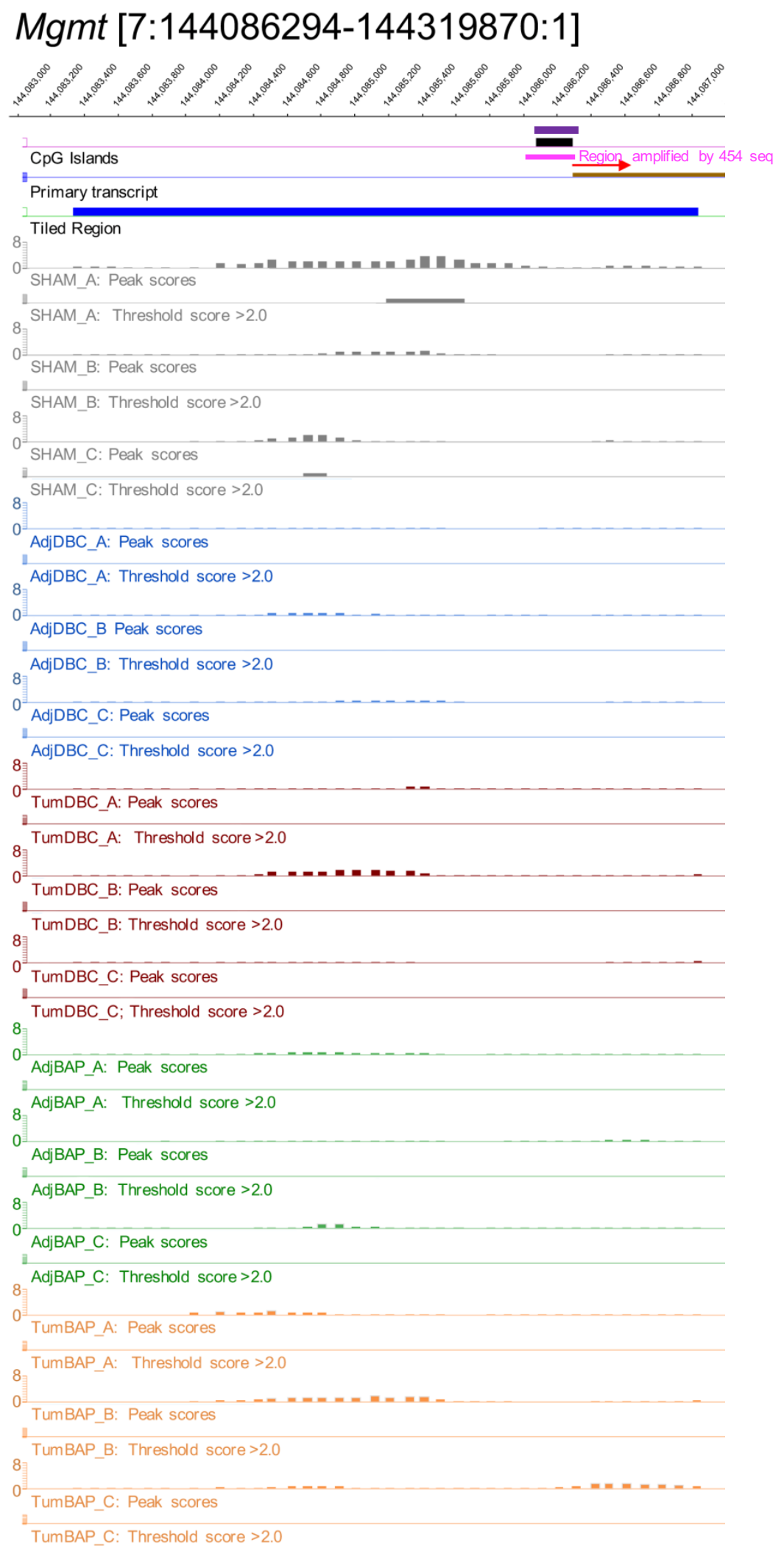


sample types (Fig. 29). Unfortunately, none of the regions for which differential methylation was observed using the genome-wide array aligned with the region analyzed by 454 sequencing, even though $\mathrm{CpG}$ islands were targeted for the ultra-deep sequencing analysis 
FIG. 29. Methylation of Rarb promoter in normal and tumor lung tissues. This figure illustrates methylation profiles for the promoter region of Rarb obtained using the NimbleGen Mouse DNA Methylation 3×720K CpG Island Plus RefSeq Promoter Array. Chromosomal location, predicted $\mathrm{CpG}$ islands and tiled regions are all mapped according to the NCBI37/mm9 mouse genome assembly. Also, $\mathrm{CpG}$ islands predicted by EMBOSS Cpgplot are shown in purple. Bright pink indicates the region of this gene analyzed by bisulfite amplicon sequencing by 454 (data shown in Fig. 11). Tracks representing control samples are shown in grey, adjacent normal DBC samples as blue, tumor DBC samples as red, adjacent normal $\mathrm{BaP}$ as green and tracks for tumor $\mathrm{BaP}$ as orange. Each sample is represented by two tracks, the peak scores and threshold score $>2.0$. The threshold score $>2.0$ track visualizes, by means of the solid bar, the region of peak scores that have surpassed the significance threshold and are thus considered to be hypermethylated. The peak score track displays the resulting score for each probe calculated as $-\log _{10}$ p-value using the one-sided Kolmogorov-Smirnov test. 


\section{Rarb [14:17263356-17651381:-1]}

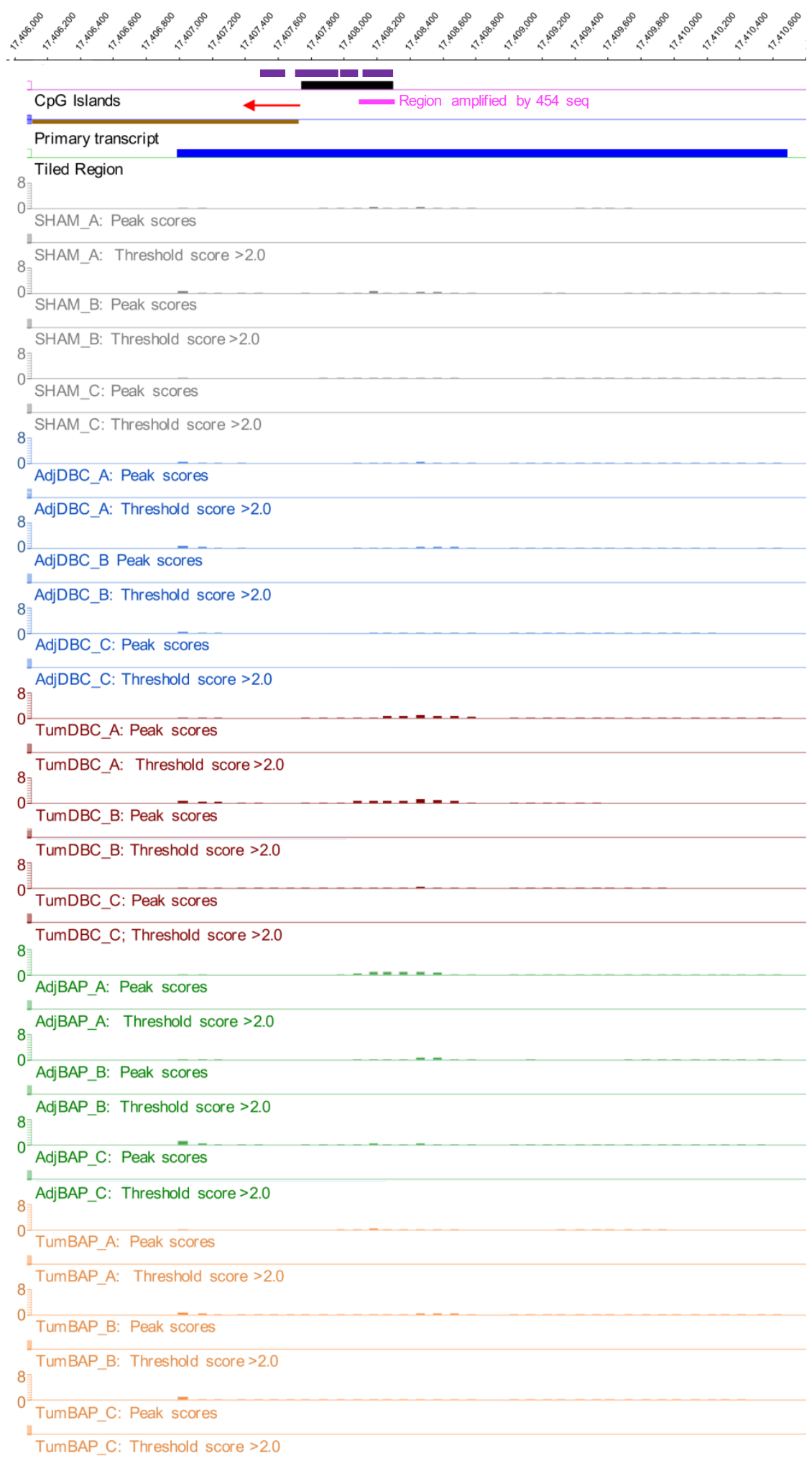




\section{DISCUSSION}

This study is the first to profile genome-wide DNA promoter methylation in lung tissues of mice exposed to environmental PAH carcinogens. Two different methodologies were employed in the investigation of promoter methylation in normal and tumor lung tissues from offspring exposed in utero to the environmental carcinogens $\mathrm{DBC}$ or $\mathrm{BaP}$. Targeted gene promoter amplicon analysis revealed consistent low methylation within the regions sequenced for all four target genes. The genome-wide assessment of CpG island promoter methylation revealed distinct patterns of methylation associated with normal and adenocarcinoma lung tissues. Gene ontology analysis revealed differences in the biological functions represented by the methylated genes in normal lung tissues compared to tumor tissues, such as cell proliferation, cell differentiation, cell cycle and growth, all of which are associated with the regulation and control of critical processes involved in lung tumorigenesis. These observations contribute to the growing knowledge that gene promoter methylation is a highly coordinated epigenetic event that is critical for the initiation and progression of lung cancer (Belinsky, 2015).

The genome-wide assessment of DNA methylation in the present model identified a methylation signature for lung tumorigenesis in $\mathrm{PAH}-$ exposed mice. A methylation profile was evident that distinguished normal lung tissue from tumor tissue obtained from mice initiated with either PAH carcinogen. Moreover, distinctions in DNA methylation were also evident when contrasting methylation profiles for tumors obtained from DBC- or BaP-initiated offspring. These distinctions were observed at the level of 
specific genes as well as in the higher order analysis of enriched GO pathways. Venn diagram analysis identified cohorts of genes uniquely methylated in tumor tissues from DBC-initiated (125 genes) or BaP-initiated (142 genes), as well as those genes that shared a pattern of hypermethylation (92 genes). The enriched GO pathways represented by gene lists from lung tumor tissue obtained from DBC- or BaP-exposed offspring (Table 9) demonstrate the differences in methylation for these exposures. Altogether, these data provide new knowledge that exposure to specific PAHs, though similar in ultimate phenotypic effect, can lead to unique epigenetic profiles in lung tumors in adult offspring.

The utilization of the two methodologies employed in the present study, genomewide and targeted amplicon methylation analysis, can lead to further functional or biological analyses of the disease state. Bisulfite amplicon sequencing provides a rich data set with $\mathrm{CpG}$ site-specific resolution for a small targeted region, approximately 300 bp. Thus, researchers must have prior knowledge or reasonable expectation that the gene targets will be differentially methylated in the assayed tissue. One may rely on evidence from the literature to select target genes for amplicon analyses, as in the case for this study. Alternatively, this prior knowledge may be gained from other methylation analysis methods, such as a genome-wide methylation array, which yields a massive amount of data useful for discovering new target genes of interest. The genome-wide methylation array may also reveal patterns of methylation between groups and provide information for use in higher order gene ontology pathway analysis. However, the use of microarrays as a first-line approach has limitations, notably with respect to their high cost, which 
necessitates limited experiment design. Furthermore, array analyses do not provide $\mathrm{CpG}$ site-specific data, rather a relative score indicative of methylation for a region of about 50 bp. When these two approaches are used in conjunction, researchers may be able to better understand the significance of the methylation profiles and their biological impact.

Based on the results obtained from analysis of the genome-wide DNA methylation data, we identified ten genes of interest for more in-depth examination of their role in carcinogenesis and evidence for methylation in lung cancer or other cancers in human or animal models. These genes include Bcl2l11, Bmpl, Fgfrlop, Hoxb1, Pdcd4, Casp7, IL11, Pten, Maea, and Tpd52l1. One should note that these genes are not necessarily representative of genome-wide methylation patterns for any particular tissue or disease state, and their selection was subjective in nature.

Bcl-2-like protein 11 (Bcl2l11) belongs to the Bcl-2 protein family of proapoptotic genes. In this study, the promoter region of Bcl2ll1 was hypermethylated in lung tumors of BaP- and DBC-initiated adult offspring. Others have reported that BCL2 was hypermethylated in human NSCLC with a methylation frequency of $42 \%$ (i.e., $42 \%$ of tumors assessed had detectable levels of methylation) (Chung, et al., 2011; Zhao et al., 2013). However, no information on methylation of Bcl2l11 in lung cancer in humans or animal models was available prior to this research. Hypermethylation of $B c l 2 l 11$ has been shown for other types of cancer, such as adenoid cystic carcinoma (Bell et al., 2011), chronic myeloid leukemia with 44\% methylation prevalence (San Jose-Eneriz et al., 2009 ) and colon cancer with $68 \%$ promoter methylation (Cho et al., 2014). Bcl2ll1 binds to anti-apoptotic proteins, which allow the proapoptotic multidomain proteins Bax and 
Bak to form channels on the mitochondrial membrane leading to cytochrome c release and apoptosis (Willis et al., 2007). Rahmani et al. suggested that Bcl2ll1 is associated with the disruption of kinase pathways in human leukemia cells leading to apoptotic signaling (Rahmani et al., 2009). Moreover, Bcl2l11 was shown to be critical for apoptosis during negative selection of autoreactive thymocytes (Bouillet et al., 2002), mature T cells (Davey et al., 2002) and B cells (Enders et al., 2003). Also, Bcl2l11 expression controlled the proliferation rate of tumor cells (Akiyama et al., 2009). Several researchers have obtained evidence suggesting that EGFR-driven NSCLC maintained a survival advantage via targeting $B c l 2 l 11$ protein for proteasomal degradation through MAPK dependent phosphorylation (Costa et al., 2007; Cragg et al., 2007; Gong et al., 2007). Promoter hypermethylation of $B c l 2 l 11$, as seen in the present study, may be advantageous for tumor cells as the proapoptotic function of $B c l 2 l 11$ is suppressed. Bone morphogenetic proteins (BMP) are secreted glycoproteins belonging to the transforming growth factor- $\beta$ super family. The present study is the first to observe hypomethylation of the Bmpl gene promoter tissues obtained from mouse lung adenocarcinoma in contrast with expansive regions of hypermethylation in normal adjacent and sham lung tissues. Other BMPs were hypomethylated in cancers, such as BMP6 in endometrial cancer (Hsu et al., 2013). Alternatively, BMP3b and BMP6 were silenced by methylation in NSCLC (Dai et al., 2001; Dai et al., 2004; Kraunz et al., 2005). Components of the BMP signaling cascade include protein kinases and transcription factors belonging to the SMAD family which transduce the BMP signal to the nucleus. The BMP gene family has been associated with an extensive range of actions 
in cancer (Bandyopadhyay et al., 2013; Hatakeyama et al., 1997). For example, activation of BMP signaling inhibits some cancer types, such as colorectal cancer, by promoting growth arrest and differentiation (Blanco Calvo et al., 2009). Additionally, abnormal BMP expression was reported in association with development and progression of cancer of the colon, stomach, prostate and lungs (Dai, et al., 2001; Dai, et al., 2004; Horvath et al., 2004; Kodach et al., 2008; Wen et al., 2006). Bone morphogenetic protein 1 is known to induce cartilage and bone formation and is a contributor to cell differentiation. In this study, $B m p l$ gene promoter regions exhibited a substantial loss of methylation in the lung tumor tissue samples in comparison to the clearly methylated normal adjacent and sham lung tissue samples. This methylation pattern is in accordance with a role for $\mathrm{Bmpl}$ in contributing to $\mathrm{PAH}$-induced tumorigenesis, possibly through induction of cell proliferation.

A similar methylation pattern was observed for fibroblast growth factor receptor 1 oncogene partner (Fgfrlop), for which the promoter region was hypermethylated in normal and normal adjacent tissues but not methylated in tumor tissues from DBC- or BaP-initiated mice. To our knowledge, this report is the first to examine methylation of Fgfrlop in lung cancer. Cortese et al. (2008) observed hypermethylation in lung cancer samples for other members of the FGFR family, such as Fgfr3, which was methylated in $100 \%$ of sampled lung adenocarcinomas and $70 \%$ of squamous cell carcinomas. Moreover, Fgfrl was associated with hypomethylation in Rhabdomyosarcoma tumors in all samples tested (Goldstein et al., 2007). Fgfrlop is a fusion partner of Fgfr1 (Popovici et al., 1999), and this chimeric protein induces myeloproliferative disease in both humans 
and mice (Guasch et al., 2004; Vizmanos et al., 2004). Gene ontology annotations related to Fgfrlop include organelle organization, localization, protein localization and macromolecular localization. Over-expression of FGFR1OP may contribute to the malignancy of human lung cancer by reduced ABL1-dependant phosphorylation of WRNIP1, resulting in cell cycle progression of lung tumors (Mano et al., 2007). A prosurvival effect of $F g f r l$ has been observed in diverse cancer cells, including leukemia, melanoma and bladder cancer (Feng et al., 2015). In the present study, the pattern of gene promoter methylation observed fit with the presumed oncogenic function of Fgfrlop. Homeobox B1 (Hoxb1) belongs to the homeobox (HOX) family of genes. The methylation pattern found in this study for Hoxbl revealed the promoter region was hypermethylated in normal sham and normal adjacent tissues, but not methylated in tumor tissues from DBC- or BaP-initiated mice. Interestingly, Hoxb1 was hypermethylated in downstream regions of the human A549 lung cancer cells, while several other HOX genes were hypermethylated in the promoter regions (Rauch et al., 2007). Furthermore, promoter hypermethylation silenced Hoxbl in acute myeloid leukemia cells, with the hypermethylated fraction of the $\mathrm{CpG}$ island being $68-97 \%$ higher in respect to normal cells (Petrini et al., 2013). In addition, researchers showed Hoxb1 to have increased methylation levels in pituitary adenomas (Duong et al., 2012) and oral squamous cell carcinoma with a mean frequency of promoter hypermethylation of $98 \%$ (Xavier et al., 2014). HOX genes encode transcription factors that are defined by the DNA-binding domain known as homeodomain (Gehring et al., 1994). In addition to their roles in development and stem cell differentiation, HOX genes are frequently 
deregulated in several different cancers including cancers of the colon, bladder, kidney, breast, prostate, thyroid, ovarian, melanoma and lung (Bhatlekar et al., 2014). AbateShen defined three categories of homeobox gene expression in tumor malignancy (AbateShen, 2002). In the first, homeobox genes that are normally only active during embryonic development are re-expressed in the neoplastic cells. This first category accounts for most cases of aberrant HOX gene expression (Abate-Shen, 2002). In the second category, homeobox genes can be expressed in malignant cells derived from tissue in which a particular gene is not expressed during embryogenesis. In the third category, homeobox genes can be down-regulated in malignant cells derived from tissues in which a particular gene is normally expressed in the fully differentiated state. When compared to AbateShen's three categories, the present study's methylation pattern for Hoxbl is most similar to the first category of homeobox gene expression. However, the pattern of aberrant expression of specific HOX genes tends to differ based on tissue type and tumor site, and consequently, more research is needed to elucidate the role Hoxbl plays in lung cancer.

The methylation profile observed from the present study of programmed cell death $4(\mathrm{Pdcd} 4)$ gene promoter DNA was hypermethylated in the lung tumor samples compared to control. No other evidence for $P d c d 4$ methylation in lung cancer has been shown to date. However, 5' $\mathrm{CpG}$ island methylation at the transcription start site of PDCD4 blocks expression at the mRNA level in human glioma cell lines (Gao et al., 2009). PDCD4 was also hypermethylated in the gene promoter of $36 \%$ of human gastric cancer samples (Cao et al., 2012). Pdcd4 encodes a nuclear/cytoplasmic phosphoprotein which modulates the transcription of specific genes by affecting the activity of certain 
transcription factors. $P d c d 4$, described as a tumor suppressor, promotes cell apoptosis, reduces tumorigenesis and inhibits cellular invasion (Lankat-Buttgereit and Goke, 2009). Thus, $P d c d 4$ down-regulation is an unfavorable prognosis in lung, colon, esophageal, ovarian and brain cancer (Chen et al., 2003; Fassan et al., 2010; Gao, et al., 2009; Mudduluru et al., 2007; Wei et al., 2009). Down regulation of $P d c d 4$ may contribute to tumor development in at least two ways. Decreased $P d c d 4$ expression increases mobility and invasiveness of tumor cells (Asangani et al., 2008; Leupold et al., 2007; Wang et al., 2008) and deregulates the cell response to DNA damage (Bitomsky et al., 2008; Singh et al., 2009). Hypermethylation of a gene promoter region, such as $P d c d 4$ in the present study, is typically correlated with down regulation of tumor suppressor gene expression which may be a contributing factor in carcinogenesis, as discussed above.

In this study, the Caspase-7 (Casp7) gene promoter was hypermethylated in only the normal lung tissue of sham animals. While limited data are available on the methylation profiles of caspase genes, Hopkins-Donaldson et al. showed that caspase- 8 was methylated in $52 \%$ of small cell lung cancers and none of the NSCLC samples (2003). Furthermore, Teitz et al. (2000) found that caspase-8 was highly methylated in neuroblastomas. Caspase-7 is an apoptosis-related cysteine peptidase and belongs to a family of cysteine proteases that participate in apoptotic and inflammatory signaling pathways (Fiandalo and Kyprianou, 2012). Because of the comparable specificities of caspases, these proteins may have some functional redundancy in vitro (Luthi and Martin, 2007). However, caspases have been shown to display distinct activities in vivo (Slee et al., 2001), which may be explained by a compensatory mechanism (Zheng et al., 2000). 
Caspase-7, along with caspase-3, is responsible for the execution of the apoptosis program through cleavage of caspase-activated DNase (Larsen et al., 2010). Caspase-7 and -3 are downstream targets for caspase-9, which becomes catalytically active following formation of the apoptosome (Ohtsuka et al., 2003). If caspase-9 is inactive, then the downstream executioner caspases' activation is inhibited (Janssen et al., 2007). Additionally, caspase- 8 is activated during apoptosis induction and cleaves caspase- 7 and -3 , leading towards apoptosis (Yuan and Kroemer, 2010). There is relatively little evidence to support a central role for a single caspase in tumorigenesis, rather, caspases may perform their functions jointly resulting in the combined effect of all caspases (Olsson and Zhivotovsky, 2011). The promoter methylation profile for Casp7 pointed to a loss of methylation in tumor tissues from PAH-initiated animals initiated samples, further research is needed to fully elucidate the role of Casp7 in lung tumorigenesis.

In this study, the promoter region of interleukin 11 (Il11) was hypermethylated in normal lung tissue of sham-initiated adult offspring. While methylation of IL11 in lung cancer has not been investigated to date, researchers studying major depressive disorder reported that the average $\mathrm{CpG}$ site methylation frequency was low in blood samples, ranging from 1\%-9\% (Powell et al., 2013). Furthermore, other interleukins have been shown to be methylated in lung cancer, such as $I L 12 R B 2$ with methylation evident in $42 \%$ of primary NSCLCs (Suzuki et al., 2007). An interplay between genetic variants and DNA methylation of IL13 was shown to influence asthma-related lung function (Patil et al., 2013). Il11 is a multifunctional cytokine that directly stimulates the proliferation of hematopoietic stem cells. Il11 plays a role in regulating cell proliferation, transcription, 
cell-cell signaling, gene expression and establishment of localization. Il11 signaling has been associated with growth of osteosarcoma (Lewis et al., 2009) and is thought to facilitate cell motility in human chondrosarcoma cells (Li et al., 2012b). Multiple researchers established that I111 plays a key role in the metastasis of breast cancer to bone (Ren et al., 2013; Ren et al., 2014; Sotiriou et al., 2001). Furthermore, Il11 has been shown to be an important factor mediating the proliferation and invasion of human colorectal cancer (Chua et al., 2013; Yamazumi et al., 2006). Along with previously mentioned cancers, Il11 expression is associated with cervical, pancreatic, prostate and liver cancers (Ernst and Putoczki, 2014). Thus, the low methylation observed for Il1 lgene promoter in the PAH-exposed offspring suggests an involvement in lung cancer development, possibly through induction of cell proliferation.

In the present study, the promoter region of phosphatase and tensin homolog (Pten) gene was hypermethylated in the lung tissues obtained from mice initiated with DBC in utero. Differences observed between DBC- and BaP-initiated tissue samples may be reflective of the different potency of these PAH compounds, as DBC is a more potent mutagen with a higher rate of DNA adduct formation. Methylation of Pten has previously been identified in lung cancer with 35\% prevalence in NSCLC samples and 69\% prevalence among the NSCLC cell lines tested (Li et al., 2012a; Maeda et al., 2015; Soria et al., 2002). Additionally, hypermethylation of the Pten promoter region has been reported in numerous cancer types, such as breast cancer with a $48 \%$ methylation prevalence (Garcia et al., 2004), glioblastomas (Wiencke et al., 2007), gastric cancer with $73 \%$ methylation prevalence (Kang et al., 2002) and endometrial cancer with 19\% 
methylation prevalence (Salvesen et al., 2001). Pten is an important tumor suppressor gene that acts through its phosphatase protein as a regulator of the cellular phosphoinositide 3-kinase signaling pathway. This pathway regulates a wide range of important biological processes. Thus, it is unsurprising that dysregulation of this signaling pathway plays a key role in several different disease states (Leslie and Downes, 2004). Gene ontology annotations related to Pten include gene expression, cell communication, response to stimulus, cell differentiation and cell death, among several others. Pten is the most frequently mutated gene in prostate cancer, yet mutation of Pten is rarely observed in lung cancer (9\%) (Chu and Tarnawski, 2004). Methylation is considered the main factor contributing to low expression of Pten in NSCLC cells (Soria, et al., 2002).

This study was the first to determine a methylation profile for macrophage erythroblast attacher (Maea) in lung cancer, and to our knowledge, no other data are available describing the methylation status of Maea in other cancers. Maea, also known as $E M P$ (erythroblast macrophage protein) or human lung cancer oncogene 10 protein, has been commonly associated with erythroblast/macrophage interactions during erythroid differentiation and enucleation of erythroblasts (Hanspal et al., 1998; Mohandas and Chasis, 2010; Soni et al., 2008). Moreover, Maea undergoes dynamic rearrangements that may be involved in cytokinesis and cell division (Bala et al., 2006). In addition, Maea is widely distributed in nonerythroid cells, such as heart, placenta, brain, liver, kidney, pancreas, skeletal muscle and lung (Hanspal, et al., 1998). Gene ontology annotations associated with Maea include cell cycle, regulation of biological quality 
control, cell differentiation, cytoskeleton organization and organelle organization. The observed low methylation profile of PAH-exposed lung tissue samples for Maea, as seen in the present study, suggest that this gene may contribute to cancer development in DBC-initiated offspring.

The present study is the first to observe a methylation profile for tumor protein D52-like 1 (Tpd52l1) in lung cancer. The promoter region of Tpd52ll was consistently hypermethylated in lung tissue obtained from all sham-initiated offspring, hypomethylated in all tissues from DBC-initiated animals and intermittently methylated in BaP-initiated mice. Tpd52l1 is a member of the tumor protein D52 (Tpd52) family and is involved in cell proliferation and calcium signaling. Byrne et al. (1995) identified the first D52-like gene, after which the Tpd52 family was identified to be contributors in several human cancers including breast cancer (Boutros and Byrne, 2005), colon cancer (Malek et al., 2002), ovarian cancer (Byrne et al., 2005), prostate cancer (Wang et al., 2004), glioma (Wang et al., 2014), melanoma (Roesch et al., 2007), and lung cancer (Chen et al., 1996). Loss of methylation of the Tpd52l1 promoter, as observed in DBCexposed offspring, may promote growth of tumor cells as loss of methylation allows for Tpd52l1 to increase proliferation.

The four target genes selected for gene promoter amplicon analysis were selected based on prior knowledge from the literature (see Table 1). Methylation analysis of four genes (Cdkn2a, Rarb, Dapk1 and Mgmt) using 454 deep bisulfite sequencing of CpG sites at single base pair resolution on targeted regions within the promoter $\mathrm{CpG}$ island revealed consistent low levels of methylation within all four genes. A conserved region of 
hypermethylation in $C d k n 2 \mathrm{a}$ amplicon was detected across all sample types, covering four $\mathrm{CpG}$ sites from $\mathrm{bp}-156$ to -169 upstream in relation to the first bp of the first exon. Although interesting, the biological significance of the elevated methylation at this location has yet to be determined. Furthermore, the approach taken in the present study was an in-depth look at single base pair resolution within a targeted region of the promoter of these four genes. Putative $\mathrm{CpG}$ island sites of the promoter regions varied in length and number between the target genes. The $\mathrm{CpG}$ island within $C d k n 2 a$ was large, beginning $-1,000 \mathrm{bp}$ upstream of the first exon and extending to about $300 \mathrm{bp}$ downstream. Rarb contained three separate $\mathrm{CpG}$ islands ranging from $-300 \mathrm{bp}$ to $100 \mathrm{bp}$ in relation to the first bp of the first exon. A single large $\mathrm{CpG}$ island surrounded Dapkl first exon beginning at -180 bp extending to 750 bp past the first exon. Finally, the $\mathrm{CpG}$ island with the promoter region of Mgmt began at $-225 \mathrm{bp}$ and extended to $25 \mathrm{bp}$, in relation to the first bp of the first exon. Given the number of sample types to be examined and the depth of sequencing desired using 454 technology, the experiment design allowed for only one region to be sequenced per gene. Thus, it is possible that other regions of the gene promoter were differentially methylated. Indeed, since this analysis was performed, new cost-effective technologies have become available that would allow for more extensive methylation analysis of the entire promoter region, while also allowing for a large number of samples to be sequenced. Our approach aimed at $\mathrm{CpG}$ site-specific methylation in an attempt to achieve a greater depth of analysis, resulting in a fine resolution of data which can only be achieved by sequencing. In addition to the amplicon sequencing, the four target genes methylation profiles were identified using the genome- 
wide assessment of DNA methylation, which corresponded with the low levels of methylation observed for the sequenced regions. Interestingly, three of the four target gene promoter regions were methylated in regions not analyzed by bisulfite sequencing. However, the methylation patterns observed for these genes differed based on tissue type and, consequently, the pattern of gene promoter methylation observed did not fit with the presumed tumor suppressor function of these cancer critical genes.

Results of this study provide further supporting evidence that DBC is a substantially more potent transplacental carcinogen when compared to BaP. Even though $\mathrm{BaP}$ was administered at a substantially higher dose than DBC, lung tumor incidence was only a fraction $(29.5 \%)$ of the rate observed for DBC-initiated offspring (100\%). A similar trend was also observed for lung tumor multiplicity. Initial studies of transplacental exposure to $\mathrm{DBC}$ and $\mathrm{BaP}$ using the cross of strain $\mathrm{B} 6129 \mathrm{SF} 1 / \mathrm{J}$ dams to 129S1/SvlmJ sires were developed by Yu et al. (2006a; 2006b). The incidence of lung tumors in initial studies employing a single high dose was $100 \%$ and the average lung tumor multiplicity was 13 to 15 per tumor-bearing animal (Castro, et al., 2008a; Castro, et al., 2008b; Castro et al., 2009; Castro, et al., 2008c; Yu, et al., 2006a; Yu, et al., 2006b). In a subsequent study, Shorey et al. (2012) developed a multiple dosing regimen resulting in reduced gross lung tumor incidence of $80 \%$ and reduced multiplicity (3 tumors per tumor-bearing animal) in comparison to previous studies. Our gross lung tumor data, utilizing the multiple dosing regimen, more closely resembled the outcome of the initial single high dose studies, with $100 \%$ tumor incidence and tumor multiplicity typically ranging from 3 to 15 tumors per tumor-bearing animal within the DBC group. 
$\mathrm{BaP}$ previously had never been used in a multiple dosing regimen, and lung tumor incidence was $30 \%$ with lung tumor multiplicity at one to two tumors per tumor-bearing animal. Although the study was not designed to compare lung tumorigenesis among males and females, we did make an interesting observation that lung tumor incidence was lower in BaP-initiated females than in males. Historically, lung cancer incidence is consistently higher in men compared to women in all populations, with sex ratios varying from about 1.5 to 20 (International Agency for Research on Cancer, 2008). Tobacco smoke is a major risk factor for lung cancer, and gender differences in lung cancer mortality reflect patterns of smoking uptake and cessation over time (American Cancer Society, 2015). However, other researchers found that women may be more susceptible to tobacco smoke and potentially more vulnerable to lung cancer (Kiyohara and Ohno, 2010). Further work is needed to understand the gender differences in susceptibility to lung cancer.

In conclusion, we identified a set of cancer-related genes with distinct methylation profiles according to tissue type using a genome-wide approach to assess DNA promoter methylation tumor tissues of offspring initiated with $\mathrm{DBC}$ or $\mathrm{BaP}$ in utero in comparison to normal lung tissue from sham-initiated offspring. These new genes of interest may be important targets for epigenetic modification during the process of lung tumorigenesis in animals exposed to environmental PAHs. However, it remains to be seen whether PAH exposure directly results in their altered methylation or their methylation state is indicative of the cancer disease state. To address this uncertainty, these newly identified target genes may be subject to 454 sequencing analysis in the same manner as the four 
tumor suppressor genes originally selected for this study. Another future application of the data from this work would be a comparison of the methylation profile obtained for PAH-initiated lung tumors in mice to methylation profile for human NSCLC. Such an analysis may provide insight into the applicability of this transplacental carcinogenesis animal model as a model for human lung cancer with respect to epigenome modifications that occur during lung carcinogenesis. In future bioinformatics analyses, we plan to address these questions by comparing the methylation profile obtained in the present study with those publicly available for human NSCLC. A greater understanding of the epigenetic events that contribute to lung tumorigenesis may enhance approaches for lung cancer prevention and treatment and may reduce the heavy burden of this devastating disease. 


\section{REFERENCES}

Abate-Shen, C. (2002). Deregulated homeobox gene expression in cancer: cause or consequence? Nat. Rev. Cancer 2, 777-785.

Akiyama, T., Dass, C. R., and Choong, P. F. (2009). Bim-targeted cancer therapy: a link between drug action and underlying molecular changes. Mol. Cancer Ther. 8, 3173-3180.

American Cancer Society (2015). Cancer Facts \& Figures. Available at: http://www.cancer.org/research/cancerfactsstatistics/cancerfactsfigures2015/.

Anderson, L. M. (2004). Introduction and overview. Perinatal carcinogenesis: growing a node for epidemiology, risk management, and animal studies. Toxicol. Appl. Pharmacol. 199, 85-90.

Anderson, L. M., Diwan, B. A., Fear, N. T., and Roman, E. (2000). Critical windows of exposure for children's health: cancer in human epidemiological studies and neoplasms in experimental animal models. Environ. Health Perspect. 108 Suppl 3, 573-594.

Armsrong, R. (1997). Glutathione-S-transferases. In Comprehensive Toxicology:

Biotransformation (F. P. Guengerich, and I. G. Sipes, Eds.), Vol. 3, pp. 307-327. Pergamon Press, Oxford, UK.

Asangani, I. A., Rasheed, S. A., Nikolova, D. A., Leupold, J. H., Colburn, N. H., Post, S., and Allgayer, H. (2008). MicroRNA-21 (miR-21) post-transcriptionally downregulates tumor suppressor Pdcd4 and stimulates invasion, intravasation and metastasis in colorectal cancer. Oncogene 27, 2128-2136. 
ATSDR (1995). Toxicological Profile for Polycyclic Aromatic Hydrocarbons. Available at: http://www.atsdr.cdc.gov.

Bach, P. B., Kattan, M. W., Thornquist, M. D., Kris, M. G., Tate, R. C., Barnett, M. J., Hsieh, L. J., and Begg, C. B. (2003). Variations in lung cancer risk among smokers. J. Natl. Cancer Inst. 95, 470-478.

Badham, H. J., LeBrun, D. P., Rutter, A., and Winn, L. M. (2010). Transplacental benzene exposure increases tumor incidence in mouse offspring: possible role of fetal benzene metabolism. Carcinogenesis 31, 1142-1148.

Bala, S., Kumar, A., Soni, S., Sinha, S., and Hanspal, M. (2006). Emp is a component of the nuclear matrix of mammalian cells and undergoes dynamic rearrangements during cell division. Biochem. Biophys. Res. Commun. 342, 1040-1048.

Bandyopadhyay, A., Yadav, P. S., and Prashar, P. (2013). BMP signaling in development and diseases: a pharmacological perspective. Biochem. Pharmacol. 85, 857-864.

Baylin, S. B., and Jones, P. A. (2007). Epigenetic determinants of cancer. In Epigenetics (C. D. Allis, T. Jenuwein, and D. Reinberg, Eds.), pp. 457-476. Cold Spring Harbor Laboratory Press, Cold Spring Harbor, N.Y.

Belinsky, S. A. (2004). Gene-promoter hypermethylation as a biomarker in lung cancer. Nature reviews. Cancer 4, 707-717.

Belinsky, S. A. (2005). Silencing of genes by promoter hypermethylation: key event in rodent and human lung cancer. Carcinogenesis 26, 1481-1487.

Belinsky, S. A. (2015). Unmasking the lung cancer epigenome. Annu. Rev. Physiol. 77, 453-474. 
Bell, A., Bell, D., Weber, R. S., and El-Naggar, A. K. (2011). CpG island methylation profiling in human salivary gland adenoid cystic carcinoma. Cancer 117, 28982909.

Bell, J. (1876). Paraffin epithelioma of the scrotum. Edinb. Med. J. 22, 135 - 137.

Bhatlekar, S., Fields, J. Z., and Boman, B. M. (2014). HOX genes and their role in the development of human cancers. J. Mol. Med. 92, 811-823.

Bird, A. (2007). Perceptions of epigenetics. Nature 447, 396-398.

Bitomsky, N., Wethkamp, N., Marikkannu, R., and Klempnauer, K. H. (2008). siRNAmediated knockdown of Pdcd4 expression causes upregulation of p21(Waf1/Cip1) expression. Oncogene 27, 4820-4829.

Blanco Calvo, M., Bolos Fernandez, V., Medina Villaamil, V., Aparicio Gallego, G., Diaz Prado, S., and Grande Pulido, E. (2009). Biology of BMP signalling and cancer. Clin. Transl. Oncol. 11, 126-137.

Bostrom, C. E., Gerde, P., Hanberg, A., Jernstrom, B., Johansson, C., Kyrklund, T., Rannug, A., Tornqvist, M., Victorin, K., and Westerholm, R. (2002). Cancer risk assessment, indicators, and guidelines for polycyclic aromatic hydrocarbons in the ambient air. Environ. Health Perspect. 110 Suppl 3, 451-488.

Bouillet, P., Purton, J. F., Godfrey, D. I., Zhang, L. C., Coultas, L., Puthalakath, H., Pellegrini, M., Cory, S., Adams, J. M., and Strasser, A. (2002). BH3-only Bcl-2 family member Bim is required for apoptosis of autoreactive thymocytes. Nature 415, 922-926. 
Boutros, R., and Byrne, J. A. (2005). D53 (TPD52L1) is a cell cycle-regulated protein maximally expressed at the G2-M transition in breast cancer cells. Exp. Cell Res. 310, 152-165.

Boyland, E., and Levi, A. A. (1935). Metabolism of polycyclic compounds: Production of dihydroxydihydroanthracene from anthracene. Biochem. J. 29, 2679-2683.

Brzezianska, E., Dutkowska, A., and Antczak, A. (2013). The significance of epigenetic alterations in lung carcinogenesis. Mol. Biol. Rep. 40, 309-325.

Burchell, B., McGurk, K., Brierley, C. H., and Clarke, D. J. (1997). UDPglucuronosyltransferases. In Comprehensive toxicology: Biotransformation (F. P. Guengerich, Ed.), Vol. 3, pp. 401-436. Pergamon Press, Oxford, UK.

Burdge, G. C., and Lillycrop, K. A. (2010). Nutrition, epigenetics, and developmental plasticity: implications for understanding human disease. Annu. Rev. Nutr. 30, 315-339.

Buterin, T., Hess, M. T., Luneva, N., Geacintov, N. E., Amin, S., Kroth, H., Seidel, A., and Naegeli, H. (2000). Unrepaired fjord region polycyclic aromatic hydrocarbonDNA adducts in ras codon 61 mutational hot spots. Cancer Res. 60, 1849-1856.

Byrne, J. A., Balleine, R. L., Schoenberg Fejzo, M., Mercieca, J., Chiew, Y. E., Livnat, Y., St Heaps, L., Peters, G. B., Byth, K., Karlan, B. Y., Slamon, D. J., Harnett, P., and Defazio, A. (2005). Tumor protein D52 (TPD52) is overexpressed and a gene amplification target in ovarian cancer. Int. J. Cancer 117, 1049-1054.

Byrne, J. A., Tomasetto, C., Garnier, J. M., Rouyer, N., Mattei, M. G., Bellocq, J. P., Rio, M. C., and Basset, P. (1995). A screening method to identify genes commonly 
overexpressed in carcinomas and the identification of a novel complementary DNA sequence. Cancer Res. 55, 2896-2903.

Cao, Z., Yoon, J. H., Nam, S. W., Lee, J. Y., and Park, W. S. (2012). PDCD4 expression inversely correlated with miR-21 levels in gastric cancers. J. Cancer Res. Clin. Oncol. 138, 611-619.

Castro, D. J., Baird, W. M., Pereira, C. B., Giovanini, J., Lohr, C. V., Fischer, K. A., Yu, Z., Gonzalez, F. J., Krueger, S. K., and Williams, D. E. (2008a). Fetal mouse Cyp 1 b1 and transplacental carcinogenesis from maternal exposure to dibenzo(a,l)pyrene. Cancer Prev. Res. 1, 128-134.

Castro, D. J., Lohr, C. V., Fischer, K. A., Pereira, C. B., and Williams, D. E. (2008b). Lymphoma and lung cancer in offspring born to pregnant mice dosed with dibenzo[a,1]pyrene: the importance of in utero vs. lactational exposure. Toxicol. Appl. Pharmacol. 233, 454-458.

Castro, D. J., Lohr, C. V., Fischer, K. A., Waters, K. M., Webb-Robertson, B. J., Dashwood, R. H., Bailey, G. S., and Williams, D. E. (2009). Identifying efficacious approaches to chemoprevention with chlorophyllin, purified chlorophylls and freeze-dried spinach in a mouse model of transplacental carcinogenesis. Carcinogenesis 30, 315-320.

Castro, D. J., Yu, Z., Lohr, C. V., Pereira, C. B., Giovanini, J. N., Fischer, K. A., Orner, G. A., Dashwood, R. H., and Williams, D. E. (2008c). Chemoprevention of dibenzo $[a, l]$ pyrene transplacental carcinogenesis in mice born to mothers administered green tea: primary role of caffeine. Carcinogenesis 29, 1581-1586. 
Centers for Disease Control and Prevention (2014). Lung cancer. Available at: http://www.cdc.gov/cancer/lung/index.htm. Accessed March 2015.

Chakravarti, D., Mailander, P. C., Cavalieri, E. L., and Rogan, E. G. (2000). Evidence that error-prone DNA repair converts dibenzo[a,1]pyrene-induced depurinating lesions into mutations: formation, clonal proliferation and regression of initiated cells carrying H-ras oncogene mutations in early preneoplasia. Mutat. Res. 456, $17-32$.

Chen, S. L., Maroulakou, I. G., Green, J. E., Romano-Spica, V., Modi, W., Lautenberger, J., and Bhat, N. K. (1996). Isolation and characterization of a novel gene expressed in multiple cancers. Oncogene 12, 741-751.

Chen, Y., Knosel, T., Kristiansen, G., Pietas, A., Garber, M. E., Matsuhashi, S., Ozaki, I., and Petersen, I. (2003). Loss of PDCD4 expression in human lung cancer correlates with tumour progression and prognosis. J. Pathol. 200, 640-646.

Cho, Y., Turner, N. D., Davidson, L. A., Chapkin, R. S., Carroll, R. J., and Lupton, J. R. (2014). Colon cancer cell apoptosis is induced by combined exposure to the n-3 fatty acid docosahexaenoic acid and butyrate through promoter methylation. Exp. Biol. Med. 239, 302-310.

Choi, Y. L., Takeuchi, K., Soda, M., Inamura, K., Togashi, Y., Hatano, S., Enomoto, M., Hamada, T., Haruta, H., Watanabe, H., Kurashina, K., Hatanaka, H., Ueno, T., Takada, S., Yamashita, Y., Sugiyama, Y., Ishikawa, Y., and Mano, H. (2008). Identification of novel isoforms of the EML4-ALK transforming gene in nonsmall cell lung cancer. Cancer Res. 68, 4971-4976. 
Choudhary, D., Jansson, I., Schenkman, J. B., Sarfarazi, M., and Stoilov, I. (2003). Comparative expression profiling of 40 mouse cytochrome P450 genes in embryonic and adult tissues. Arch. Biochem. Biophys. 414, 91-100.

Choudhary, D., Jansson, I., Stoilov, I., Sarfarazi, M., and Schenkman, J. B. (2005). Expression patterns of mouse and human CYP orthologs (families 1-4) during development and in different adult tissues. Arch. Biochem. Biophys. 436, 50-61.

Chu, E. C., and Tarnawski, A. S. (2004). PTEN regulatory functions in tumor suppression and cell biology. Med. Sci. Monit. 10, RA235-241.

Chua, A. C., Klopcic, B. R., Ho, D. S., Fu, S. K., Forrest, C. H., Croft, K. D., Olynyk, J. K., Lawrance, I. C., and Trinder, D. (2013). Dietary iron enhances colonic inflammation and IL-6/IL-11-Stat3 signaling promoting colonic tumor development in mice. PLoS One 8, e78850.

Chung, J. H., Lee, H. J., Kim, B. H., Cho, N. Y., and Kang, G. H. (2011). DNA methylation profile during multistage progression of pulmonary adenocarcinomas. Virchows Arch. 459, 201-211.

Conney, A. H. (1982). Induction of microsomal enzymes by foreign chemicals and carcinogenesis by polycyclic aromatic hydrocarbons: GHA Clowes Memorial Lecture. Cancer Res. 42, 4875-4917.

Cook, J., Hewett, C., and Hieger, I. (1933). The isolation of a cancer-producing hydrocarbon from coal tar. Parts I, II and III. J. Chem. Soc. Paper 106, 395-405. 
Cortese, R., Hartmann, O., Berlin, K., and Eckhardt, F. (2008). Correlative gene expression and DNA methylation profiling in lung development nominate new biomarkers in lung cancer. Int. J. Biochem. Cell Biol. 40, 1494-1508.

Costa, D. B., Halmos, B., Kumar, A., Schumer, S. T., Huberman, M. S., Boggon, T. J., Tenen, D. G., and Kobayashi, S. (2007). BIM mediates EGFR tyrosine kinase inhibitor-induced apoptosis in lung cancers with oncogenic EGFR mutations. PLoS Med. 4, 1669-1679.

Couraud, S., Zalcman, G., Milleron, B., Morin, F., and Souquet, P. J. (2012). Lung cancer in never smokers--a review. Eur. J. Cancer 48, 1299-1311.

Cragg, M. S., Kuroda, J., Puthalakath, H., Huang, D. C., and Strasser, A. (2007). Gefitinib-induced killing of NSCLC cell lines expressing mutant EGFR requires BIM and can be enhanced by BH3 mimetics. PLoS Med. 4, 1681-1689.

Cui, X., Wakai, T., Shirai, Y., Hatakeyama, K., and Hirano, S. (2006). Chronic oral exposure to inorganic arsenate interferes with methylation status of p16INK4a and RASSF1A and induces lung cancer in A/J mice. Toxicol. Sci. 91, 372-381.

Dai, Z., Lakshmanan, R. R., Zhu, W. G., Smiraglia, D. J., Rush, L. J., Fruhwald, M. C., Brena, R. M., Li, B., Wright, F. A., Ross, P., Otterson, G. A., and Plass, C. (2001). Global methylation profiling of lung cancer identifies novel methylated genes. Neoplasia 3, 314-323.

Dai, Z., Popkie, A. P., Zhu, W. G., Timmers, C. D., Raval, A., Tannehill-Gregg, S., Morrison, C. D., Auer, H., Kratzke, R. A., Niehans, G., Amatschek, S., Sommergruber, W., Leone, G. W., Rosol, T., Otterson, G. A., and Plass, C. 
(2004). Bone morphogenetic protein 3B silencing in non-small-cell lung cancer. Oncogene 23, 3521-3529.

Damiani, L. A., Yingling, C. M., Leng, S., Romo, P. E., Nakamura, J., and Belinsky, S. A. (2008). Carcinogen-induced gene promoter hypermethylation is mediated by DNMT1 and causal for transformation of immortalized bronchial epithelial cells. Cancer Res. 68, 9005-9014.

Davey, G. M., Kurts, C., Miller, J. F., Bouillet, P., Strasser, A., Brooks, A. G., Carbone, F. R., and Heath, W. R. (2002). Peripheral deletion of autoreactive CD8 T cells by cross presentation of self-antigen occurs by a Bcl-2-inhibitable pathway mediated by Bim. J. Exp. Med. 196, 947-955.

Delage, B., and Dashwood, R. H. (2008). Dietary manipulation of histone structure and function. Annu. Rev. Nutr. 28, 347-366.

Denison, M. S., Pandini, A., Nagy, S. R., Baldwin, E. P., and Bonati, L. (2002). Ligand binding and activation of the Ah receptor. Chem. Biol. Interact. 141, 3-24.

Du, Z., Zhou, X., Ling, Y., Zhang, Z., and Su, Z. (2010). agriGO: a GO analysis toolkit for the agricultural community. Nucleic Acids Res. 38, W64-70.

Duffel, M. W. (1997). Sulfotransferases. In Comprehensive Toxicology: Biotransformation (F. P. Guengerich, Ed.), Vol. 3, pp. 365-384. Pergamon Press, Oxford, UK.

Dumitrescu, R. G. (2012). Epigenetic markers of early tumor development. Methods Mol. Biol. 863, 3-14. 
Duong, C. V., Emes, R. D., Wessely, F., Yacqub-Usman, K., Clayton, R. N., and Farrell, W. E. (2012). Quantitative, genome-wide analysis of the DNA methylome in sporadic pituitary adenomas. Endocr. Relat. Cancer 19, 805-816.

Enders, A., Bouillet, P., Puthalakath, H., Xu, Y., Tarlinton, D. M., and Strasser, A. (2003). Loss of the pro-apoptotic BH3-only Bcl-2 family member Bim inhibits BCR stimulation-induced apoptosis and deletion of autoreactive B cells. J. Exp. Med. 198, 1119-1126.

Ernst, M., and Putoczki, T. L. (2014). Molecular pathways: IL11 as a tumor-promoting cytokine-translational implications for cancers. Clin. Cancer Res. 20, 5579-5588.

Esteller, M., Hamilton, S. R., Burger, P. C., Baylin, S. B., and Herman, J. G. (1999). Inactivation of the DNA repair gene O6-methylguanine-DNA methyltransferase by promoter hypermethylation is a common event in primary human neoplasia. Cancer Res. 59, 793-797.

Fang, N., Gu, J., Wei, H., You, J., and Zhou, Q. (2014). [A meta-analysis of Association between MGMT gene promoter methylation and non-small cell lung cancer]. Zhongguo Fei Ai Za Zhi 17, 601-605.

Fassan, M., Cagol, M., Pennelli, G., Rizzetto, C., Giacomelli, L., Battaglia, G., Zaninotto, G., Ancona, E., Ruol, A., and Rugge, M. (2010). Programmed cell death 4 protein in esophageal cancer. Oncol. Rep. 24, 135-139.

Feinberg, A. P., Ohlsson, R., and Henikoff, S. (2006). The epigenetic progenitor origin of human cancer. Nat. Rev. Genet 7, 21-33. 
Feng, S., Zhou, L., Nice, E. C., and Huang, C. (2015). Fibroblast growth factor receptors: multifactorial-contributors to tumor initiation and progression. Histol. Histopathol. 30, 13-31.

Ferlay, J., Soerjomataram, I., Ervik, M., Dikshit, R., Eser, S., Mathers, C., Rebelo, M., Parkin, D., Forman, D., and Bray, F. (2013). GLOBOCAN 2012 v1.0, Cancer Incidence and Mortality Worldwide. IARC CancerBase No. 11. Available at: http://globocan.iarc.fr. Accessed November 2014.

Fiandalo, M. V., and Kyprianou, N. (2012). Caspase control: protagonists of cancer cell apoptosis. Exp. Oncol. 34, 165-175.

Fischer, J. R., Ohnmacht, U., Rieger, N., Zemaitis, M., Stoffregen, C., Manegold, C., and Lahm, H. (2007). Prognostic significance of RASSF1A promoter methylation on survival of non-small cell lung cancer patients treated with gemcitabine. Lung Cancer 56, 115-123.

Fish, T. (2015a). DNA methylation in lung tissues of mouse offspring exposed in utero to polycyclic aromatic hydrocarbons: Appendix A. NimbleGen processed data report for all methylated peaks MS, Utah State University, Logan, UT. http://dx.doi.org/10.15142/T35P49

Fish, T. (2015b). DNA methylation in lung tissues of mouse offspring exposed in utero to polycyclic aromatic hydrocarbons: Appendix B. NimbleGen processed data report for all nearest peaks MS, Utah State University, Logan, UT. http://dx.doi.org/10.15142/T3201B 
Fish, T. (2015c). DNA methylation in lung tissues of mouse offspring exposed in utero to polycyclic aromatic hydrocarbons: Appendix C. Peak scores summary table for all methylated genes in any data set. MS, Utah State University, Logan, UT. http://dx.doi.org/10.15142/T3X59V

Fish, T. (2015d). DNA methylation in lung tissues of mouse offspring exposed in utero to polycyclic aromatic hydrocarbons: Appendix D. Gene lists for ontology analyses. MS, Utah State University, Logan, UT. http://dx.doi.org/10.15142/T3SG6K

Fish, T. (2015e). DNA methylation in lung tissues of mouse offspring exposed in utero to polycyclic aromatic hydrocarbons: Appendix F. Gene ontology maps. MS, Utah State University, Logan, UT. http://dx.doi.org/10.15142/T3NP4N

Flicek, P., Amode, M. R., Barrell, D., Beal, K., Brent, S., Carvalho-Silva, D., Clapham, P., Coates, G., Fairley, S., Fitzgerald, S., Gil, L., Gordon, L., Hendrix, M., Hourlier, T., Johnson, N., Kahari, A. K., Keefe, D., Keenan, S., Kinsella, R., Komorowska, M., Koscielny, G., Kulesha, E., Larsson, P., Longden, I., McLaren, W., Muffato, M., Overduin, B., Pignatelli, M., Pritchard, B., Riat, H. S., Ritchie, G. R., Ruffier, M., Schuster, M., Sobral, D., Tang, Y. A., Taylor, K., Trevanion, S., Vandrovcova, J., White, S., Wilson, M., Wilder, S. P., Aken, B. L., Birney, E., Cunningham, F., Dunham, I., Durbin, R., Fernandez-Suarez, X. M., Harrow, J., Herrero, J., Hubbard, T. J., Parker, A., Proctor, G., Spudich, G., Vogel, J., Yates, A., Zadissa, A., and Searle, S. M. (2012). Ensembl 2012. Nucleic Acids Res. 40 , D84-90. 
Flower, K. B., Hoppin, J. A., Lynch, C. F., Blair, A., Knott, C., Shore, D. L., and Sandler, D. P. (2004). Cancer risk and parental pesticide application in children of Agricultural Health Study participants. Environ. Health Perspect. 112, 631-635.

Freedman, N. D., Leitzmann, M. F., Hollenbeck, A. R., Schatzkin, A., and Abnet, C. C. (2008). Cigarette smoking and subsequent risk of lung cancer in men and women: analysis of a prospective cohort study. Lancet Oncol. 9, 649-656.

Furonaka, O., Takeshima, Y., Awaya, H., Kushitani, K., Kohno, N., and Inai, K. (2005). Aberrant methylation and loss of expression of O-methylguanine-DNA methyltransferase in pulmonary squamous cell carcinoma and adenocarcinoma. Pathol. Int. 55, 303-309.

Gao, F., Wang, X., Zhu, F., Wang, Q., Zhang, X., Guo, C., Zhou, C., Ma, C., Sun, W., Zhang, Y., Chen, Y. H., and Zhang, L. (2009). PDCD4 gene silencing in gliomas is associated with 5'CpG island methylation and unfavourable prognosis. J. Cell. Mol. Med. 13, 4257-4267.

Garcia, J. M., Silva, J., Pena, C., Garcia, V., Rodriguez, R., Cruz, M. A., Cantos, B., Provencio, M., Espana, P., and Bonilla, F. (2004). Promoter methylation of the PTEN gene is a common molecular change in breast cancer. Genes Chromosomes Cancer 41, 117-124.

Geacintov, N. E., Broyde, S., Buterin, T., Naegeli, H., Wu, M., Yan, S., and Patel, D. J. (2002). Thermodynamic and structural factors in the removal of bulky DNA adducts by the nucleotide excision repair machinery. Biopolymers $\mathbf{6 5}, \mathbf{2 0 2 - 2 1 0 .}$ 
Gehring, W. J., Affolter, M., and Burglin, T. (1994). Homeodomain proteins. Annu. Rev. Biochem. 63, 487-526.

Goldstein, M., Meller, I., and Orr-Urtreger, A. (2007). FGFR1 over-expression in primary rhabdomyosarcoma tumors is associated with hypomethylation of a $5^{\prime}$ $\mathrm{CpG}$ island and abnormal expression of the AKT1, NOG, and BMP4 genes. Genes Chromosomes Cancer 46, 1028-1038.

Gong, Y., Somwar, R., Politi, K., Balak, M., Chmielecki, J., Jiang, X., and Pao, W. (2007). Induction of BIM is essential for apoptosis triggered by EGFR kinase inhibitors in mutant EGFR-dependent lung adenocarcinomas. PLoS Med. 4, e294.

Guasch, G., Delaval, B., Arnoulet, C., Xie, M. J., Xerri, L., Sainty, D., Birnbaum, D., and Pebusque, M. J. (2004). FOP-FGFR1 tyrosine kinase, the product of a $\mathrm{t}(6 ; 8)$ translocation, induces a fatal myeloproliferative disease in mice. Blood 103, 309312.

Guenthner, T. M., and Oesch, F. (1981). Microsomal epoxide hydrolase and its role in polycyclic aromatic hydrocarbon biotransformation. In Polycyclic Aromatic Hydrocarbons and Cancer (H. V. Gelboin, and P. O. Ts'o, Eds.), Vol. 3, pp. 183212. Academic Press, New York.

Guo, M., Alumkal, J., Drachova, T., Gao, D., Marina, S. S., Jen, J., and Herman, J. G. (2015). CHFR methylation strongly correlates with methylation of DNA damage repair and apoptotic pathway genes in non-small cell lung cancer. Discov. Med. 19, 151-158. 
Hakkola, J., Pasanen, M., Pelkonen, O., Hukkanen, J., Evisalmi, S., Anttila, S., Rane, A., Mantyla, M., Purkunen, R., Saarikoski, S., Tooming, M., and Raunio, H. (1997). Expression of CYP1B1 in human adult and fetal tissues and differential inducibility of CYP1B1 and CYP1A1 by Ah receptor ligands in human placenta and cultured cells. Carcinogenesis 18, 391-397.

Hall, M., and Grover, P. (1990). Polycyclic aromatic hydrocarbons: metabolism, activation and tumour initiation. In Chemical Carcinogenesis and Mutagenesis I (C. S. Cooper, and P. L. Grover, Eds.), pp. 327-372. Springer.

Hall, T. A. (1999). BioEdit: a user-friendly biological sequence alignment editor and analysis program for Windows 95/98/NT. Nucleic Acids Symp. Ser. 41, 95-98.

Hanahan, D., and Weinberg, R. A. (2000). The hallmarks of cancer. Cell 100, 57-70.

Hanahan, D., and Weinberg, R. A. (2011). Hallmarks of cancer: the next generation. Cell $144,646-674$.

Hanspal, M., Smockova, Y., and Uong, Q. (1998). Molecular identification and functional characterization of a novel protein that mediates the attachment of erythroblasts to macrophages. Blood 92, 2940-2950.

Harvey, R. G. (1991). Molecular preoperties of polyarenes. In Polycyclic Aromatic Hydrocarbons: Chemistry and Carcinogenicity (R. G. Harvey, Ed.), pp. 96-116. Cambridge University Press, Cambridge, UK.

Hatakeyama, S., Gao, Y. H., Ohara-Nemoto, Y., Kataoka, H., and Satoh, M. (1997). Expression of bone morphogenetic proteins of human neoplastic epithelial cells. Biochem. Mol. Biol. Int. 42, 497-505. 
Hecht, S. S. (2011). More than 500 trillion molecules of strong carcinogens per cigarette: use in product labelling? Tob. Control 20, 387.

Henry, S. A. (1947). Occupational cutaneous cancer attributable to certain chemicals in industry. Br. Med. Bull. 4, 389-401.

Henschke, C. I., and Miettinen, O. S. (2004). Women's susceptibility to tobacco carcinogens. Lung Cancer 43, 1-5.

Henschke, C. I., Yip, R., and Miettinen, O. S. (2006). Women's susceptibility to tobacco carcinogens and survival after diagnosis of lung cancer. JAMA 296, 180-184.

Herman, J. G., and Baylin, S. B. (2003). Gene silencing in cancer in association with promoter hypermethylation. N. Engl. J. Med. 349, 2042-2054.

Hernandez-Boussard, T. M., and Hainaut, P. (1998). A specific spectrum of p53 mutations in lung cancer from smokers: review of mutations compiled in the IARC p53 database. Environ. Health Perspect. 106, 385-391.

Hieger, I. (1930). The spectra of cancer-producing tars and oils and of related substances. Biochem. J. 24, 505-511.

Higginbotham, S., RamaKrishna, N. V., Johansson, S. L., Rogan, E. G., and Cavalieri, E. L. (1993). Tumor-initiating activity and carcinogenicity of dibenzo[a,l]pyrene versus 7,12-dimethylbenz $[a]$ anthracene and benzo $[a]$ pyrene at low doses in mouse skin. Carcinogenesis 14, 875-878.

Hopkins-Donaldson, S., Ziegler, A., Kurtz, S., Bigosch, C., Kandioler, D., Ludwig, C., Zangemeister-Wittke, U., and Stahel, R. (2003). Silencing of death receptor and 
caspase- 8 expression in small cell lung carcinoma cell lines and tumors by DNA methylation. Cell Death Differ. 10, 356-364.

Horvath, L. G., Henshall, S. M., Kench, J. G., Turner, J. J., Golovsky, D., Brenner, P. C., O'Neill, G. F., Kooner, R., Stricker, P. D., Grygiel, J. J., and Sutherland, R. L. (2004). Loss of BMP2, Smad8, and Smad4 expression in prostate cancer progression. Prostate 59, 234-242.

Howlader N, N. A., Krapcho M, Garshell J, Miller D, Altekruse SF, Kosary CL, Yu M, Ruhl J, Tatalovich Z,Mariotto A, Lewis DR, Chen HS, Feuer EJ, Cronin KA (2015). SEER Cancer Statistics Review, 1975-2012. Available at: http://seer.cancer.gov/csr/1975_2012/. Accessed June 2015.

Hsu, Y. T., Gu, F., Huang, Y. W., Liu, J., Ruan, J., Huang, R. L., Wang, C. M., Chen, C. L., Jadhav, R. R., Lai, H. C., Mutch, D. G., Goodfellow, P. J., Thompson, I. M., Kirma, N. B., and Huang, T. H. (2013). Promoter hypomethylation of EpCAMregulated bone morphogenetic protein gene family in recurrent endometrial cancer. Clin. Cancer Res. 19, 6272-6285.

Huberman, E., Sachs, L., Yang, S. K., and Gelboin, V. (1976). Identification of mutagenic metabolites of benzo (a) pyrene in mammalian cells. Proc. Natl. Acad. Sci. USA 73, 607-611.

International Agency for Research on Cancer (2008). Cancer incidence in five continents. Volume IX. IARC Sci. Publ., 1-837. 
International Agency for Research on Cancer (2010). Some non-heterocyclic polycyclic aromatic hydrocarbons and some related exposures. IARC Monogr. Eval. Carcinog. Risks Hum. 92, 1-853.

International Agency for Research on Cancer (2012). Chemical agents and related occupations. IARC Monogr. Eval. Carcinog. Risks Hum. 100, 9-562.

Iwanari, M., Nakajima, M., Kizu, R., Hayakawa, K., and Yokoi, T. (2002). Induction of CYP1A1, CYP1A2, and CYP1B1 mRNAs by nitropolycyclic aromatic hydrocarbons in various human tissue-derived cells: chemical-, cytochrome P450 isoform-, and cell-specific differences. Arch. Toxicol. 76, 287-298.

Janssen, K., Pohlmann, S., Janicke, R. U., Schulze-Osthoff, K., and Fischer, U. (2007). Apaf-1 and caspase-9 deficiency prevents apoptosis in a Bax-controlled pathway and promotes clonogenic survival during paclitaxel treatment. Blood 110,36623672.

Jensen, C. D., Block, G., Buffler, P., Ma, X., Selvin, S., and Month, S. (2004). Maternal dietary risk factors in childhood acute lymphoblastic leukemia (United States). Cancer Causes Control 15, 559-570.

Jones, P. A., and Baylin, S. B. (2002). The fundamental role of epigenetic events in cancer. Nat. Rev. Genet 3, 415-428.

Kang, Y. H., Lee, H. S., and Kim, W. H. (2002). Promoter methylation and silencing of PTEN in gastric carcinoma. Lab. Invest. 82, 285-291.

Kennaway, E. L. (1930). Further experiments on cancer-producing substances. Biochem. J. 24, 497-504. 
Kennaway, E. L., and Hieger, I. (1930). Carcinogenic substances and their fluorescence spectra. Br. Med. J. 1, 1044-1046.

Kim, D. H., Nelson, H. H., Wiencke, J. K., Christiani, D. C., Wain, J. C., Mark, E. J., and Kelsey, K. T. (2001a). Promoter methylation of DAP-kinase: association with advanced stage in non-small cell lung cancer. Oncogene 20, 1765-1770.

Kim, D. H., Nelson, H. H., Wiencke, J. K., Zheng, S., Christiani, D. C., Wain, J. C., Mark, E. J., and Kelsey, K. T. (2001b). p16(INK4a) and histology-specific methylation of $\mathrm{CpG}$ islands by exposure to tobacco smoke in non-small cell lung cancer. Cancer Res. 61, 3419-3424.

Kim, J. H., Stansbury, K. H., Walker, N. J., Trush, M. A., Strickland, P. T., and Sutter, T. R. (1998). Metabolism of benzo[a]pyrene and benzo[a]pyrene-7,8-diol by human cytochrome P450 1B1. Carcinogenesis 19, 1847-1853.

Kiyohara, C., and Ohno, Y. (2010). Sex differences in lung cancer susceptibility: a review. Gend. Med. 7, 381-401.

Kodach, L. L., Wiercinska, E., de Miranda, N. F., Bleuming, S. A., Musler, A. R., Peppelenbosch, M. P., Dekker, E., van den Brink, G. R., van Noesel, C. J., Morreau, H., Hommes, D. W., Ten Dijke, P., Offerhaus, G. J., and Hardwick, J. C. (2008). The bone morphogenetic protein pathway is inactivated in the majority of sporadic colorectal cancers. Gastroenterology 134, 1332-1341.

Kraunz, K. S., Nelson, H. H., Liu, M., Wiencke, J. K., and Kelsey, K. T. (2005). Interaction between the bone morphogenetic proteins and Ras/MAP-kinase signalling pathways in lung cancer. Br. J. Cancer 93, 949-952. 
Lankat-Buttgereit, B., and Goke, R. (2009). The tumour suppressor Pdcd4: recent advances in the elucidation of function and regulation. Biol. Cell 101, 309-317.

Larsen, B. D., Rampalli, S., Burns, L. E., Brunette, S., Dilworth, F. J., and Megeney, L. A. (2010). Caspase 3/caspase-activated DNase promote cell differentiation by inducing DNA strand breaks. Proc. Natl. Acad. Sci. USA 107, 4230-4235.

Le Calvez, F., Mukeria, A., Hunt, J. D., Kelm, O., Hung, R. J., Taniere, P., Brennan, P., Boffetta, P., Zaridze, D. G., and Hainaut, P. (2005). TP53 and KRAS mutation load and types in lung cancers in relation to tobacco smoke: distinct patterns in never, former, and current smokers. Cancer Res. 65, 5076-5083.

Leslie, N. R., and Downes, C. P. (2004). PTEN function: how normal cells control it and tumour cells lose it. Biochem. J. 382, 1-11.

Leupold, J. H., Yang, H. S., Colburn, N. H., Asangani, I., Post, S., and Allgayer, H. (2007). Tumor suppressor Pdcd4 inhibits invasion/intravasation and regulates urokinase receptor (u-PAR) gene expression via Sp-transcription factors. Oncogene 26, 4550-4562.

Lewis, V. O., Ozawa, M. G., Deavers, M. T., Wang, G., Shintani, T., Arap, W., and Pasqualini, R. (2009). The interleukin-11 receptor alpha as a candidate liganddirected target in osteosarcoma: consistent data from cell lines, orthotopic models, and human tumor samples. Cancer Res. 69, 1995-1999.

Li, G., Zhao, J., Peng, X., Liang, J., Deng, X., and Chen, Y. (2012a). The mechanism involved in the loss of PTEN expression in NSCLC tumor cells. Biochem. Biophys. Res. Commun. 418, 547-552. 
Li, L. C., and Dahiya, R. (2002). MethPrimer: designing primers for methylation PCRs. Bioinformatics 18, 1427-1431.

Li, T. M., Wu, C. M., Huang, H. C., Chou, P. C., Fong, Y. C., and Tang, C. H. (2012b). Interleukin-11 increases cell motility and up-regulates intercellular adhesion molecule-1 expression in human chondrosarcoma cells. J. Cell. Biochem. 113, 3353-3362.

Li, W., Cowley, A., Uludag, M., Gur, T., McWilliam, H., Squizzato, S., Park, Y. M., Buso, N., and Lopez, R. (2015). The EMBL-EBI bioinformatics web and programmatic tools framework. Nucleic Acids Res. 43, W580-584.

Licchesi, J. D., Westra, W. H., Hooker, C. M., and Herman, J. G. (2008). Promoter hypermethylation of hallmark cancer genes in atypical adenomatous hyperplasia of the lung. Clin. Cancer Res. 14, 2570-2578.

Lightfoot, T. J., and Roman, E. (2004). Causes of childhood leukaemia and lymphoma. Toxicol. Appl. Pharmacol. 199, 104-117.

Luch, A. (2005). Polycyclic aromatic hydrocarbon-induced carcinogenesis - an introduction. In The Carcinogenic Effects of Polycyclic Aromatic Hydrocarbons (A. Luch, Ed.), pp. 1-18. Imperial College Press, London.

Luch, A., Coffing, S. L., Tang, Y. M., Schneider, A., Soballa, V., Greim, H., Jefcoate, C. R., Seidel, A., Greenlee, W. F., Baird, W. M., and Doehmer, J. (1998). Stable expression of human cytochrome P450 1B1 in V79 Chinese hamster cells and metabolically catalyzed DNA adduct formation of dibenzo[a,l]pyrene. Chem. Res. Toxicol. 11, 686-695. 
Luger, K., Rechsteiner, T. J., Flaus, A. J., Waye, M. M., and Richmond, T. J. (1997). Characterization of nucleosome core particles containing histone proteins made in bacteria. J. Mol. Biol. 272, 301-311.

Lukas, J., Parry, D., Aagaard, L., Mann, D. J., Bartkova, J., Strauss, M., Peters, G., and Bartek, J. (1995). Retinoblastoma-protein-dependent cell-cycle inhibition by the tumour suppressor p16. Nature 375, 503-506.

Luthi, A. U., and Martin, S. J. (2007). The CASBAH: a searchable database of caspase substrates. Cell Death Differ. 14, 641-650.

Ma, X., Buffler, P. A., Gunier, R. B., Dahl, G., Smith, M. T., Reinier, K., and Reynolds, P. (2002). Critical windows of exposure to household pesticides and risk of childhood leukemia. Environ. Health Perspect. 110, 955-960.

MacKinnon, A. C., Kopatz, J., and Sethi, T. (2010). The molecular and cellular biology of lung cancer: identifying novel therapeutic strategies. Br. Med. Bull. 95, 47-61. Maeda, M., Murakami, Y., Watari, K., Kuwano, M., Izumi, H., and Ono, M. (2015). CpG hypermethylation contributes to decreased expression of PTEN during acquired resistance to gefitinib in human lung cancer cell lines. Lung Cancer 87, 265-271.

Mahadevan, B., Courter, L. A., and Baird, W. M. (2007). Mutations induced by polycyclic aromatic hydrocarbons. In New Developments in Mutation Research (C. L. Valon, Ed.), pp. 19-40. Nova Publishers, New York.

Malek, R. L., Irby, R. B., Guo, Q. M., Lee, K., Wong, S., He, M., Tsai, J., Frank, B., Liu, E. T., Quackenbush, J., Jove, R., Yeatman, T. J., and Lee, N. H. (2002). 
Identification of Src transformation fingerprint in human colon cancer. Oncogene 21, 7256-7265.

Mano, Y., Takahashi, K., Ishikawa, N., Takano, A., Yasui, W., Inai, K., Nishimura, H., Tsuchiya, E., Nakamura, Y., and Daigo, Y. (2007). Fibroblast growth factor receptor 1 oncogene partner as a novel prognostic biomarker and therapeutic target for lung cancer. Cancer Sci. 98, 1902-1913.

McKinnon, R. A., Hall, P. d. 1. M., Quattrochi, L. C., Tukey, R. H., and McManus, M. E. (1991). Localization of CYP1A1 and CYP1A2 messenger RNA in normal human liver and in hepatocellular carcinoma by in situ hybridization. Hepatology 14, 848-856.

Merlo, A., Herman, J. G., Mao, L., Lee, D. J., Gabrielson, E., Burger, P. C., Baylin, S. B., and Sidransky, D. (1995). 5' CpG island methylation is associated with transcriptional silencing of the tumour suppressor p16/CDKN2/MTS1 in human cancers. Nat. Med. 1, 686-692.

Mohandas, N., and Chasis, J. A. (2010). The erythroid niche: molecular processes occurring within erythroblastic islands. Transfus. Clin. Biol. 17, 110-111.

Molina, J. R., Yang, P., Cassivi, S. D., Schild, S. E., and Adjei, A. A. (2008). Non-small cell lung cancer: epidemiology, risk factors, treatment, and survivorship. Mayo Clin. Proc. 83, 584-594.

Mudduluru, G., Medved, F., Grobholz, R., Jost, C., Gruber, A., Leupold, J. H., Post, S., Jansen, A., Colburn, N. H., and Allgayer, H. (2007). Loss of programmed cell death 4 expression marks adenoma-carcinoma transition, correlates inversely with 
phosphorylated protein kinase $\mathrm{B}$, and is an independent prognostic factor in resected colorectal cancer. Cancer 110, 1697-1707.

National Cancer Institute (2015). Non-small cell lung cancer treatment. Available at: http://www.cancer.gov/types/lung/patient/non-small-cell-lung-treatment-pdq section/. Accessed March 2015.

Nelson, D. R., Koymans, L., Kamataki, T., Stegeman, J. J., Feyereisen, R., Waxman, D. J., Waterman, M. R., Gotoh, O., Coon, M. J., and Estabrook, R. W. (1996). P450 superfamily: update on new sequences, gene mapping, accession numbers and nomenclature. Pharmacogenetics 6, 1-42.

Newbold, R. F., and Brookes, P. (1976). Exceptional mutagenicity of a benzo[a]pyrene diol epoxide in cultured mammalian cells. Nature 261, 52-54.

Nichols, L., Saunders, R., and Knollmann, F. D. (2012). Causes of death of patients with lung cancer. Arch. Pathol. Lab. Med. 136, 1552-1557.

Nicolov, I. G., and Chernozemsky, I. N. (1979). Tumors and hyperplastic lesions in Syrian hamsters following transplacental and neonatal treatment with cigarette smoke condensate. J. Cancer Res. Clin. Oncol. 94, 249-256.

Ohtsuka, T., Buchsbaum, D., Oliver, P., Makhija, S., Kimberly, R., and Zhou, T. (2003). Synergistic induction of tumor cell apoptosis by death receptor antibody and chemotherapy agent through $\mathrm{JNK} / \mathrm{p} 38$ and mitochondrial death pathway. Oncogene 22, 2034-2044.

Olsson, M., and Zhivotovsky, B. (2011). Caspases and cancer. Cell Death Differ. 18, 1441-1449. 
Osann, K. E., Anton-Culver, H., Kurosaki, T., and Taylor, T. (1993). Sex differences in lung-cancer risk associated with cigarette smoking. Int. J. Cancer 54, 44-48.

Passey, R., and Carter-Braine, J. (1925). Experimental soot cancer. J. Pathol. Bacteriol. 28, 133-144.

Patel, A. C., Anna, C. H., Foley, J. F., Stockton, P. S., Tyson, F. L., Barrett, J. C., and Devereux, T. R. (2000). Hypermethylation of the p16 (Ink4a) promoter in B6C3F1 mouse primary lung adenocarcinomas and mouse lung cell lines. Carcinogenesis 21, 1691-1700.

Patil, V. K., Holloway, J. W., Zhang, H., Soto-Ramirez, N., Ewart, S., Arshad, S. H., and Karmaus, W. (2013). Interaction of prenatal maternal smoking, interleukin 13 genetic variants and DNA methylation influencing airflow and airway reactivity. Clin. Epigenetics 5, 22.

Pendharkar, D., Ausekar, B. V., and Gupta, S. (2013). Molecular biology of lung cancera review. Indian J. Surg. Oncol. 4, 120-124.

Perera, F. P., Jedrychowski, W., Rauh, V., and Whyatt, R. M. (1999). Molecular epidemiologic research on the effects of environmental pollutants on the fetus. Environ. Health Perspect. 107 Suppl 3, 451-460.

Perera, F. P., Tang, D., Rauh, V., Lester, K., Tsai, W. Y., Tu, Y. H., Weiss, L., Hoepner, L., King, J., Del Priore, G., and Lederman, S. A. (2005). Relationships among polycyclic aromatic hydrocarbon-DNA adducts, proximity to the World Trade Center, and effects on fetal growth. Environ. Health Perspect. 113, 1062-1067. 
Perera, F. P., Tang, D., Tu, Y. H., Cruz, L. A., Borjas, M., Bernert, T., and Whyatt, R. M. (2004). Biomarkers in maternal and newborn blood indicate heightened fetal susceptibility to procarcinogenic DNA damage. Environ. Health Perspect. 112, 1133-1136.

Petrini, M., Felicetti, F., Bottero, L., Errico, M. C., Morsilli, O., Boe, A., De Feo, A., and Care, A. (2013). HOXB1 restored expression promotes apoptosis and differentiation in the HL60 leukemic cell line. Cancer Cell Int. 13, 101.

Phillips, D. H. (1983). Fifty years of benzo(a)pyrene. Nature 303, 468-472.

Phillips, D. H. (1999). Polycyclic aromatic hydrocarbons in the diet. Mutat. Res.-Genet. Toxicol. Environ. Mutag. 443, 139-147.

Popovici, C., Zhang, B., Gregoire, M. J., Jonveaux, P., Lafage-Pochitaloff, M., Birnbaum, D., and Pebusque, M. J. (1999). The t(6;8)(q27;p11) translocation in a stem cell myeloproliferative disorder fuses a novel gene, FOP, to fibroblast growth factor receptor 1. Blood $\mathbf{9 3}, 1381-1389$.

Pott, P. (1775). Chirurgical Observations Relative to the Cataract, The Polypus of the Nose, the Cancer of the Scrotum, the Different Kind of Ruptures, and the Mortification of the Toes and Feet. Printed by T.J. Carnegy for L. Hawes, W. Clarke and R. Collins, London.

Powell, T. R., Smith, R. G., Hackinger, S., Schalkwyk, L. C., Uher, R., McGuffin, P., Mill, J., and Tansey, K. E. (2013). DNA methylation in interleukin-11 predicts clinical response to antidepressants in GENDEP. Transl. Psychiatry 3, e300. 
Pulling, L. C., Divine, K. K., Klinge, D. M., Gilliland, F. D., Kang, T., Schwartz, A. G., Bocklage, T. J., and Belinsky, S. A. (2003). Promoter hypermethylation of the O6-methylguanine-DNA methyltransferase gene: more common in lung adenocarcinomas from never-smokers than smokers and associated with tumor progression. Cancer Res. 63, 4842-4848.

Pulling, L. C., Vuillemenot, B. R., Hutt, J. A., Devereux, T. R., and Belinsky, S. A. (2004). Aberrant promoter hypermethylation of the death-associated protein kinase gene is early and frequent in murine lung tumors induced by cigarette smoke and tobacco carcinogens. Cancer Res. 64, 3844-3848.

Rahmani, M., Anderson, A., Habibi, J. R., Crabtree, T. R., Mayo, M., Harada, H., Ferreira-Gonzalez, A., Dent, P., and Grant, S. (2009). The BH3-only protein Bim plays a critical role in leukemia cell death triggered by concomitant inhibition of the PI3K/Akt and MEK/ERK1/2 pathways. Blood 114, 4507-4516.

Rauch, T., Wang, Z., Zhang, X., Zhong, X., Wu, X., Lau, S. K., Kernstine, K. H., Riggs, A. D., and Pfeifer, G. P. (2007). Homeobox gene methylation in lung cancer studied by genome-wide analysis with a microarray-based methylated $\mathrm{CpG}$ island recovery assay. Proc. Natl. Acad. Sci. USA 104, 5527-5532.

Ren, L., Wang, X., Dong, Z., Liu, J., and Zhang, S. (2013). Bone metastasis from breast cancer involves elevated IL-11 expression and the gp130/STAT3 pathway. Med. Oncol. 30, 634. 
Ren, L., Yu, Y., Wang, X., Ge, J., and Wen, L. (2014). Levels and clinical significances of interleukin 11 in breast tissue and serum of bone metastasis of breast cancer [Article in Chinese]. Zhonghua Yi Xue Za Zhi 94, 2656-2660.

Risch, H. A., Howe, G. R., Jain, M., Burch, J. D., Holowaty, E. J., and Miller, A. B. (1993). Are female smokers at higher risk for lung cancer than male smokers? A case-control analysis by histologic type. Am. J. Epidemiol. 138, 281-293.

Roesch, A., Becker, B., Bentink, S., Spang, R., Vogl, A., Hagen, I., Landthaler, M., and Vogt, T. (2007). Ataxia telangiectasia-mutated gene is a possible biomarker for discrimination of infiltrative deep penetrating nevi and metastatic vertical growth phase melanoma. Cancer Epidemiol. Biomarkers Prev. 16, 2486-2490.

Rohde, C., Zhang, Y., Reinhardt, R., and Jeltsch, A. (2010). BISMA--fast and accurate bisulfite sequencing data analysis of individual clones from unique and repetitive sequences. BMC Bioinformatics 11, 230.

Rozan, L. M., and El-Deiry, W. S. (2007). p53 downstream target genes and tumor suppression: a classical view in evolution. Cell Death Differ. 14, 3-9.

Saarikoski, S. T., Husgafvel-Pursiainen, K., Hirvonen, A., Vainio, H., Gonzalez, F. J., and Anttila, S. (1998). Localization of CYP1A1 mRNA in human lung by in situ hybridization: comparison with immunohistochemical findings. Int. J. Cancer 77, 33-39.

Sadikovic, B., Andrews, J., Carter, D., Robinson, J., and Rodenhiser, D. I. (2008). Genome-wide H3K9 histone acetylation profiles are altered in benzopyrenetreated MCF7 breast cancer cells. J. Biol. Chem. 283, 4051-4060. 
Sadikovic, B., and Rodenhiser, D. I. (2006). Benzopyrene exposure disrupts DNA methylation and growth dynamics in breast cancer cells. Toxicol. Appl. Pharmacol. 216, 458-468.

Salvesen, H. B., MacDonald, N., Ryan, A., Jacobs, I. J., Lynch, E. D., Akslen, L. A., and Das, S. (2001). PTEN methylation is associated with advanced stage and microsatellite instability in endometrial carcinoma. Int. J. Cancer 91, 22-26.

San Jose-Eneriz, E., Agirre, X., Jimenez-Velasco, A., Cordeu, L., Martin, V., Arqueros, V., Garate, L., Fresquet, V., Cervantes, F., Martinez-Climent, J. A., Heiniger, A., Torres, A., Prosper, F., and Roman-Gomez, J. (2009). Epigenetic down-regulation of BIM expression is associated with reduced optimal responses to imatinib treatment in chronic myeloid leukaemia. Eur. J. Cancer 45, 1877-1889.

Sandler, D. P., Everson, R. B., Wilcox, A. J., and Browder, J. P. (1985). Cancer risk in adulthood from early life exposure to parents' smoking. Am. J. Public Health 75, 487-492.

Sasco, A. J., and Vainio, H. (1999). From in utero and childhood exposure to parental smoking to childhood cancer: a possible link and the need for action. Hum. Exp. Toxicol. 18, 192-201.

Schlessinger, J. (2002). Ligand-induced, receptor-mediated dimerization and activation of EGF receptor. Cell 110, 669-672.

Shen, H., Huang, Y., Wang, R., Zhu, D., Li, W., Shen, G., Wang, B., Zhang, Y., Chen, Y., Lu, Y., Chen, H., Li, T., Sun, K., Li, B., Liu, W., Liu, J., and Tao, S. (2013). 
Global atmospheric emissions of polycyclic aromatic hydrocarbons from 1960 to 2008 and future predictions. Environ. Sci. Technol. 47, 6415-6424.

Shimada, T., and Fujii-Kuriyama, Y. (2004). Metabolic activation of polycyclic aromatic hydrocarbons to carcinogens by cytochromes P450 1A1 and 1B1. Cancer Sci. 95, $1-6$.

Shorey, L. E., Castro, D. J., Baird, W. M., Siddens, L. K., Lohr, C. V., Matzke, M. M., Waters, K. M., Corley, R. A., and Williams, D. E. (2012). Transplacental carcinogenesis with dibenzo[def,p]chrysene (DBC): timing of maternal exposures determines target tissue response in offspring. Cancer Lett. 317, 49-55.

Siegel, R., Ma, J. M., Zou, Z. H., and Jemal, A. (2014). Cancer Statistics, 2014. CA. Cancer J. Clin. 64, 9-29.

Singh, P., Marikkannu, R., Bitomsky, N., and Klempnauer, K. H. (2009). Disruption of the Pdcd4 tumor suppressor gene in chicken DT40 cells reveals its role in the DNA-damage response. Oncogene 28, 3758-3764.

Slee, E. A., Adrain, C., and Martin, S. J. (2001). Executioner caspase-3, -6, and -7 perform distinct, non-redundant roles during the demolition phase of apoptosis. $J$. Biol. Chem. 276, 7320-7326.

Somogyi, A., and Beck, H. (1993). Nurturing and breast-feeding: exposure to chemicals in breast milk. Environ. Health Perspect. 101 Suppl 2, 45-52.

Soni, S., Bala, S., and Hanspal, M. (2008). Requirement for erythroblast-macrophage protein (Emp) in definitive erythropoiesis. Blood Cells. Mol. Dis. 41, 141-147. 
Soria, J. C., Lee, H. Y., Lee, J. I., Wang, L., Issa, J. P., Kemp, B. L., Liu, D. D., Kurie, J. M., Mao, L., and Khuri, F. R. (2002). Lack of PTEN expression in non-small cell lung cancer could be related to promoter methylation. Clin. Cancer Res. 8, 11781184.

Sotiriou, C., Lacroix, M., Lespagnard, L., Larsimont, D., Paesmans, M., and Body, J. J. (2001). Interleukins-6 and -11 expression in primary breast cancer and subsequent development of bone metastases. Cancer Lett. 169, 87-95.

Sun, S., Schiller, J. H., and Gazdar, A. F. (2007). Lung cancer in never smokers--a different disease. Nat. Rev. Cancer 7, 778-790.

Sutter, T. R., Tang, Y. M., Hayes, C. L., Wo, Y. Y., Jabs, E. W., Li, X., Yin, H., Cody, C. W., and Greenlee, W. F. (1994). Complete cDNA sequence of a human dioxininducible mRNA identifies a new gene subfamily of cytochrome P450 that maps to chromosome 2. J. Biol. Chem. 269, 13092-13099.

Suzuki, M., Iizasa, T., Nakajima, T., Kubo, R., Iyoda, A., Hiroshima, K., Nakatani, Y., and Fujisawa, T. (2007). Aberrant methylation of IL-12Rbeta2 gene in lung adenocarcinoma cells is associated with unfavorable prognosis. Ann. Surg. Oncol. 14, 2636-2642.

Tam, A. S., Devereux, T. R., Patel, A. C., Foley, J. F., Maronpot, R. R., and Massey, T. E. (2003). Perturbations of the Ink4a/Arf gene locus in aflatoxin B1-induced mouse lung tumors. Carcinogenesis 24, 121-132. 
Tang, D., Li, T. Y., Liu, J. J., Chen, Y. H., Qu, L., and Perera, F. (2006). PAH-DNA adducts in cord blood and fetal and child development in a Chinese cohort. Environ. Health Perspect. 114, 1297-1300.

Tang, X., Khuri, F. R., Lee, J. J., Kemp, B. L., Liu, D., Hong, W. K., and Mao, L. (2000). Hypermethylation of the death-associated protein (DAP) kinase promoter and aggressiveness in stage I non-small-cell lung cancer. J. Natl. Cancer Inst. 92, 1511-1516.

Teitz, T., Wei, T., Valentine, M. B., Vanin, E. F., Grenet, J., Valentine, V. A., Behm, F. G., Look, A. T., Lahti, J. M., and Kidd, V. J. (2000). Caspase 8 is deleted or silenced preferentially in childhood neuroblastomas with amplification of MYCN. Nat. Med. 6, 529-535.

Thun, M. J., Hannan, L. M., Adams-Campbell, L. L., Boffetta, P., Buring, J. E., Feskanich, D., Flanders, W. D., Jee, S. H., Katanoda, K., Kolonel, L. N., Lee, I. M., Marugame, T., Palmer, J. R., Riboli, E., Sobue, T., Avila-Tang, E., Wilkens, L. R., and Samet, J. M. (2008). Lung cancer occurrence in never-smokers: an analysis of 13 cohorts and 22 cancer registry studies. PLoS Med. 5, e185.

Thun, M. J., Henley, S. J., Burns, D., Jemal, A., Shanks, T. G., and Calle, E. E. (2006). Lung cancer death rates in lifelong nonsmokers. J. Natl. Cancer Inst. 98, 691-699.

Thun, M. J., Henley, S. J., and Calle, E. E. (2002). Tobacco use and cancer: an epidemiologic perspective for geneticists. Oncogene 21, 7307-7325. 
Timp, W., and Feinberg, A. P. (2013). Cancer as a dysregulated epigenome allowing cellular growth advantage at the expense of the host. Nat. Rev. Cancer 13, 497510.

Toyooka, S., Maruyama, R., Toyooka, K. O., McLerran, D., Feng, Z., Fukuyama, Y., Virmani, A. K., Zochbauer-Muller, S., Tsukuda, K., Sugio, K., Shimizu, N., Shimizu, K., Lee, H., Chen, C. Y., Fong, K. M., Gilcrease, M., Roth, J. A., Minna, J. D., and Gazdar, A. F. (2003a). Smoke exposure, histologic type and geography-related differences in the methylation profiles of non-small cell lung cancer. Int. J. Cancer 103, 153-160.

Toyooka, S., Toyooka, K. O., Miyajima, K., Reddy, J. L., Toyota, M., Sathyanarayana, U. G., Padar, A., Tockman, M. S., Lam, S., Shivapurkar, N., and Gazdar, A. F. (2003b). Epigenetic down-regulation of death-associated protein kinase in lung cancers. Clin. Cancer Res. 9, 3034-3041.

Tsou, J. A., Hagen, J. A., Carpenter, C. L., and Laird-Offringa, I. A. (2002). DNA methylation analysis: a powerful new tool for lung cancer diagnosis. Oncogene 21, 5450-5461.

Virmani, A. K., Rathi, A., Zochbauer-Muller, S., Sacchi, N., Fukuyama, Y., Bryant, D., Maitra, A., Heda, S., Fong, K. M., Thunnissen, F., Minna, J. D., and Gazdar, A. F. (2000). Promoter methylation and silencing of the retinoic acid receptor-beta gene in lung carcinomas. J. Natl. Cancer Inst. 92, 1303-1307.

Vizmanos, J. L., Hernandez, R., Vidal, M. J., Larrayoz, M. J., Odero, M. D., Marin, J., Ardanaz, M. T., Calasanz, M. J., and Cross, N. C. (2004). Clinical variability of 
patients with the $\mathrm{t}(6 ; 8)(\mathrm{q} 27 ; \mathrm{p} 12)$ and FGFR1OP-FGFR1 fusion: two further cases. Hematol. J. 5, 534-537.

Volkmann, R. (1874). Ueber Theer-und Russkrebs [About tar and soot cancer]. Berl. Klin. Wochenschr. 11, 218.

Vuillemenot, B. R., Pulling, L. C., Palmisano, W. A., Hutt, J. A., and Belinsky, S. A. (2004). Carcinogen exposure differentially modulates RAR-beta promoter hypermethylation, an early and frequent event in mouse lung carcinogenesis. Carcinogenesis 25, 623-629.

Waalkes, M. P., Liu, J., and Diwan, B. A. (2007). Transplacental arsenic carcinogenesis in mice. Toxicol. Appl. Pharmacol. 222, 271-280.

Wakelee, H. A., Chang, E. T., Gomez, S. L., Keegan, T. H., Feskanich, D., Clarke, C. A., Holmberg, L., Yong, L. C., Kolonel, L. N., Gould, M. K., and West, D. W. (2007). Lung cancer incidence in never smokers. J. Clin. Oncol. 25, 472-478.

Wang, Q., Sun, Z., and Yang, H. S. (2008). Downregulation of tumor suppressor Pdcd4 promotes invasion and activates both beta-catenin/Tcf and AP-1-dependent transcription in colon carcinoma cells. Oncogene 27, 1527-1535.

Wang, R., Xu, J., Saramaki, O., Visakorpi, T., Sutherland, W. M., Zhou, J., Sen, B., Lim, S. D., Mabjeesh, N., Amin, M., Dong, J. T., Petros, J. A., Nelson, P. S., Marshall, F. F., Zhau, H. E., and Chung, L. W. (2004). PrLZ, a novel prostate-specific and androgen-responsive gene of the TPD52 family, amplified in chromosome 8q21.1 and overexpressed in human prostate cancer. Cancer Res. 64, 1589-1594. 
Wang, Z., Sun, J., Zhao, Y., Guo, W., Lv, K., and Zhang, Q. (2014). Lentivirus-mediated knockdown of tumor protein D52-like 2 inhibits glioma cell proliferation. Cell. Mol. Biol. 60, 39-44.

Wei, N. A., Liu, S. S., Leung, T. H., Tam, K. F., Liao, X. Y., Cheung, A. N., Chan, K. K., and Ngan, H. Y. (2009). Loss of Programmed cell death 4 (Pdcd4) associates with the progression of ovarian cancer. Mol. Cancer $\mathbf{8}, 70$.

Weinberg, R. A. (1995). The retinoblastoma protein and cell cycle control. Cell 81, 323330.

Weinstein, I. B., Jeffrey, A. M., Jennette, K. W., Blobstein, S. H., Harvey, R. G., Harris, C., Autrup, H., Kasai, H., and Nakanishi, K. (1976). Benzo(a)pyrene diol epoxides as intermediates in nucleic acid binding in vitro and in vivo. Science 193, 592-595.

Wen, X. Z., Akiyama, Y., Baylin, S. B., and Yuasa, Y. (2006). Frequent epigenetic silencing of the bone morphogenetic protein 2 gene through methylation in gastric carcinomas. Oncogene 25, 2666-2673.

Whitlock, J. P., Jr. (1999). Induction of cytochrome P4501A1. Annu. Rev. Pharmacol. Toxicol. 39, 103-125.

Whyatt, R. M., Jedrychowski, W., Hemminki, K., Santella, R. M., Tsai, W. Y., Yang, K., and Perera, F. P. (2001). Biomarkers of polycyclic aromatic hydrocarbon-DNA damage and cigarette smoke exposures in paired maternal and newborn blood samples as a measure of differential susceptibility. Cancer Epidemiol. Biomarkers Prev. 10, 581-588. 
Whyatt, R. M., Perera, F. P., Jedrychowski, W., Santella, R. M., Garte, S., and Bell, D. A. (2000). Association between polycyclic aromatic hydrocarbon-DNA adduct levels in maternal and newborn white blood cells and glutathione S-transferase P1 and CYP1A1 polymorphisms. Cancer Epidemiol. Biomarkers Prev. 9, 207-212.

Wiencke, J. K., Zheng, S., Jelluma, N., Tihan, T., Vandenberg, S., Tamguney, T., Baumber, R., Parsons, R., Lamborn, K. R., Berger, M. S., Wrensch, M. R., HaasKogan, D. A., and Stokoe, D. (2007). Methylation of the PTEN promoter defines low-grade gliomas and secondary glioblastoma. Neuro Oncol. 9, 271-279.

Willis, S. N., Fletcher, J. I., Kaufmann, T., van Delft, M. F., Chen, L., Czabotar, P. E., Ierino, H., Lee, E. F., Fairlie, W. D., Bouillet, P., Strasser, A., Kluck, R. M., Adams, J. M., and Huang, D. C. (2007). Apoptosis initiated when BH3 ligands engage multiple Bcl-2 homologs, not Bax or Bak. Science 315, 856-859.

Wu, M., Yan, S., Patel, D. J., Geacintov, N. E., and Broyde, S. (2002). Relating repair susceptibility of carcinogen-damaged DNA with structural distortion and thermodynamic stability. Nucleic Acids Res. 30, 3422-3432.

Xavier, F. C., Destro, M. F., Duarte, C. M., and Nunes, F. D. (2014). Epigenetic repression of HOXB cluster in oral cancer cell lines. Arch. Oral Biol. 59, 783789.

Yamagiwa, K., and Ichikawa, K. (1918). Experimental study of the pathogenesis of carcinoma. J. Cancer Res. 3, 1-29.

Yamazumi, K., Nakayama, T., Kusaba, T., Wen, C. Y., Yoshizaki, A., Yakata, Y., Nagayasu, T., and Sekine, I. (2006). Expression of interleukin-11 and interleukin- 
11 receptor alpha in human colorectal adenocarcinoma; immunohistochemical analyses and correlation with clinicopathological factors. World J. Gastroenterol. 12, 317-321.

Yu, Z., Loehr, C. V., Fischer, K. A., Louderback, M. A., Krueger, S. K., Dashwood, R. H., Kerkvliet, N. I., Pereira, C. B., Jennings-Gee, J. E., Dance, S. T., Miller, M. S., Bailey, G. S., and Williams, D. E. (2006a). In utero exposure of mice to dibenzo[ $[a, l]$ pyrene produces lymphoma in the offspring: role of the aryl hydrocarbon receptor. Cancer Res. 66, 755-762.

Yu, Z., Mahadevan, B., Lohr, C. V., Fischer, K. A., Louderback, M. A., Krueger, S. K., Pereira, C. B., Albershardt, D. J., Baird, W. M., Bailey, G. S., and Williams, D. E. (2006b). Indole-3-carbinol in the maternal diet provides chemoprotection for the fetus against transplacental carcinogenesis by the polycyclic aromatic hydrocarbon dibenzo[a,l]pyrene. Carcinogenesis 27, 2116-2123.

Yuan, J., and Kroemer, G. (2010). Alternative cell death mechanisms in development and beyond. Genes Dev. 24, 2592-2602.

Zahm, S. H., and Ward, M. H. (1998). Pesticides and childhood cancer. Environ. Health Perspect. 106 Suppl 3, 893-908.

Zang, E. A., and Wynder, E. L. (1996). Differences in lung cancer risk between men and women: examination of the evidence. J. Natl. Cancer Inst. 88, 183-192.

Zhao, Y., Zhou, H., Ma, K., Sun, J., Feng, X., Geng, J., Gu, J., Wang, W., Zhang, H., He, Y., Guo, S., Zhou, X., Yu, J., and Lin, Q. (2013). Abnormal methylation of seven 
genes and their associations with clinical characteristics in early stage non-small cell lung cancer. Oncol. Letters 5, 1211-1218.

Zheng, T. S., Hunot, S., Kuida, K., Momoi, T., Srinivasan, A., Nicholson, D. W., Lazebnik, Y., and Flavell, R. A. (2000). Deficiency in caspase-9 or caspase-3 induces compensatory caspase activation. Nat. Med. 6, 1241-1247.

Zochbauer-Muller, S., Fong, K. M., Virmani, A. K., Geradts, J., Gazdar, A. F., and Minna, J. D. (2001). Aberrant promoter methylation of multiple genes in nonsmall cell lung cancers. Cancer Res. 61, 249-255. 
APPENDICES 
Appendix A

NimbleGen Processed Data Report for All Methylated Peaks.

The complete data set for all detected peaks on the NimbleGen mouse methylation array is provided as a digital data set accessible at the DOI link in the following reference (Fish, 2015a). The .zip archive includes 15 individual digital Microsoft Excel documents, one for each sample hybridized to the NimbleGen mouse methylation array. Names of the individual Excel documents are provided in Table A.1, and these documents were named according to the sample type (Sham, AdjDBC, TumDBC, AdjBaP and TumBaP) and replicate (A, B and C). Table A.2 provides a description of the file content according to the spreadsheet column title.

TABLE A.1

List of File Names Included in the Archive File for Appendix A

Sham_A_553635A01_ratio_peaks_mapToFeatures_All_Peaks.xls
Sham_B_553565A03_ratio_peaks_mapToFeatures_All_Peaks.xls
Sham_C_553574A01_ratio_peaks_mapToFeatures_All_Peaks.xls
AdjDBC_A_553634A03_ratio_peaks_mapToFeatures_All_Peaks.xls
AdjDBC_B_553654A03_ratio_peaks_mapToFeatures_All_Peaks.xls
AdjDBC_C_553635A02_ratio_peaks_mapToFeatures_All_Peaks.xls
TumDBC_A_553634A01_ratio_peaks_mapToFeatures_A1l_Peaks.xls
TumDBC_B_553565A01_ratio_peaks_mapToFeatures_All_Peaks.xls
TumDBC_C_553653A03_ratio_peaks_mapToFeatures_All_Peaks.xls
AdjBaP_A_553653A02_ratio_peaks_mapToFeatures_All_Peaks.xls
AdjBaP_B_553653A01_ratio_peaks_mapToFeatures_All_Peaks.xls
AdjBaP_C_553565A02_ratio_peaks_mapToFeatures_All_Peaks.xls
TumBaP_A_553635A03_ratio_peaks_mapToFeatures_All_Peaks.xls
TumBaP_B_553574A03_ratio_peaks_mapToFeatures_All_Peaks.xls
TumBaP_C_554845A01_ratio_peaks_mapToFeatures_All_Peaks.xls


TABLE A.2

Definitions of File Contents for Appendix A by Spreadsheet Column Title

\begin{tabular}{|c|c|}
\hline Column & Description \\
\hline PEAK_ID & An ID for each mapped peak \\
\hline CHROMOSOME & Chromosome associated with peak \\
\hline PEAK START & First base of the peak on the chromosome \\
\hline PEAK END & Last base of the peak on the chromosome \\
\hline PEAK SCORE & Average $-\log _{10} p$-value for probes in the peak region \\
\hline FEATŪRE_TRACK & $\begin{array}{l}\text { The annotation track against which peaks were mapped, } \\
\text { which is the transcription start site for this data set }\end{array}$ \\
\hline FEATURE_STRAND & Strand of the transcript \\
\hline FEATURE_START & $\begin{array}{l}\text { First base of the feature on the chromosome } \\
\text { Note: For the transcription start site, feature size is } 1 \text {; } \\
\text { therefore, start and end positions are the same. }\end{array}$ \\
\hline FEATURE_END & Last base of the feature on the chromosome \\
\hline $\begin{array}{l}\text { FEATURE_TO_PEAK_ } \\
\text { DISTANCE }\end{array}$ & Distance from center of peak to the feature \\
\hline Parent & $\begin{array}{l}\text { Internal identification number of the transcript from which the } \\
\text { transcript start site is generated }\end{array}$ \\
\hline Accession & NCBI RefSeq Accession ID \\
\hline Name & Gene symbol of the transcript \\
\hline Description & Full gene name of the transcript \\
\hline Ncbi_gene_id & NCBI Entrez GeneID of the transcript \\
\hline $\begin{array}{l}\text { Synonyms } \\
\text { attr1 (etc.) }\end{array}$ & $\begin{array}{l}\text { Other alias symbol(s) of the transcript } \\
\text { Other available attributes for the transcript }\end{array}$ \\
\hline
\end{tabular}


Appendix B

NimbleGen Processed Data Report for Nearest Methylated Peaks.

The complete data set for peaks nearest to the transcription start site on the NimbleGen mouse methylation array is provided as a digital data set accessible at the DOI link in the following reference (Fish, 2015b). The .zip archive includes 15 individual digital Microsoft Excel documents, one for each sample hybridized to the NimbleGen mouse methylation array. Names of the individual Excel documents are provided in Table B.1, and these documents were named according to the sample type (Sham, AdjDBC, TumDBC, AdjBaP and TumBaP) and replicate (A, B and C). Table B.2 provides a description of the file content according to the spreadsheet column title.

TABLE B.1

List of File Names Included in the Archive File for Appendix B

Sham_A_553635A01_ratio_peaks_mapToFeatures_Nearest_Peak.xls
Sham_B_553565A03_ratio_peaks_mapToFeatures_Nearest_Peak.xls
Sham_C_553574A01_ratio_peaks_mapToFeatures_Nearest_Peak.xls
AdjDBC_A_553634A03_ratio_peaks_mapToFeatures_Nearest_Peak.xls
AdjDBC_B_553654A03_ratio_peaks_mapToFeatures_Nearest_Peak.xls
AdjDBC_C_553635A02_ratio_peaks_mapToFeatures_Nearest_Peak.xls
TumDBC_A_553634A01_ratio_peaks_mapToFeatures_Nearest_Peak.xls
TumDBC_B_553565A01_ratio_peaks_mapToFeatures_Nearest_Peak.xls
TumDBC_C_553653A03_ratio_peaks_mapToFeatures_Nearest_Peak.xls
AdjBaP_A_553653A02_ratio_peaks_mapToFeatures_Nearest_Peak.xls
AdjBaP_B_553653A01_ratio_peaks_mapToFeatures_Nearest_Peak.xls
AdjBaP_C_553565A02_ratio_peaks_mapToFeatures_Nearest_Peak.xls
TumBaP_A_553635A03_ratio_peaks_mapToFeatures_Nearest_Peak.xls
TumBaP_B_553574A03_ratio_peaks_mapToFeatures_Nearest_Peak.xls
TumBaP_C_554845A01_ratio_peaks_mapToFeatures_Nearest_Peak.xls


TABLE B.2

Definitions of File Contents for Appendix B by Spreadsheet Column Title

\begin{tabular}{|c|c|}
\hline Column & Description \\
\hline PEAK_ID & An ID for each mapped peak \\
\hline CHROMOSOME & Chromosome associated with peak \\
\hline PEAK_START & First base of the peak on the chromosome \\
\hline PEAK END & Last base of the peak on the chromosome \\
\hline PEAK SCORE & Average $-\log _{10} p$-value for probes in the peak region \\
\hline FEATŪRE_TRACK & $\begin{array}{l}\text { The annotation track against which peaks were mapped, which } \\
\text { is the transcription start site for this data set }\end{array}$ \\
\hline FEATURE_STRAND & Strand of the transcript \\
\hline \multirow[t]{2}{*}{ FEATURE_START } & First base of the feature on the chromosome \\
\hline & $\begin{array}{l}\text { Note: For the transcription start site, feature size is } 1 \text {; therefore, } \\
\text { start and end positions are the same. }\end{array}$ \\
\hline FEATURE_END & Last base of the feature on the chromosome \\
\hline $\begin{array}{l}\text { FEATURE_TO_PEAK } \\
\text { DISTANCE }\end{array}$ & Distance from center of peak to the feature \\
\hline Parent & $\begin{array}{l}\text { Internal identification number of the transcript from which the } \\
\text { transcript start site is generated }\end{array}$ \\
\hline Accession & NCBI RefSeq Accession ID \\
\hline Name & Gene symbol of the transcript \\
\hline Description & Full gene name of the transcript \\
\hline Ncbi_gene_id & NCBI Entrez GenelD of the transcript \\
\hline Synonyms & Other alias symbol(s) of the transcript \\
\hline attr1 (etc.) & Other available attributes for the transcript \\
\hline
\end{tabular}


Appendix C

Peak Scores Summary Table for All Methylated Genes in Any Data Set.

A summary table of peak scores for the nearest peak to the indicated transcript for any peak significantly methylated (score $\geq 2.0$ ) in any of the 15 samples analyzed are provided as a Microsoft Excel file available at a DOI link in the following reference (Fish, 2015c). Table C.1 provides a description of the file content according to the spreadsheet column title.

TABLE C.1

Definitions of File Contents for Appendix C by Spreadsheet Column Title

\begin{tabular}{ll}
\hline Column & Description \\
\hline MGI Gene & Mouse Genome Informatics (MGI) Accession number \\
NCBI gene ID & NCBI Entrez GeneID of the transcript \\
Symbol & Gene symbol of the transcript \\
Name & Full gene name of the transcript \\
Peak Score & Average $-\log _{10}$ p-value for probes in the peak region \\
\hline
\end{tabular}


Appendix D

Gene Lists for Ontology Analyses

Table D.1 indicates the specific comparisons performed to generate gene lists for ontology analysis and the number of genes in each resulting data set. These lists are provided as a Microsoft Excel file available at the DOI link included in the following reference (Fish, 2015d). Within this spreadsheet, each column contains a set of gene accession numbers (MGI accession) representing genes methylated in one (or more) tissue type(s) and not in others according to the comparisons outlined in Table D.1.

TABLE D.1

\section{List of Comparisons Among Tissue Types to Generate Gene Lists for Ontology Analyses}

\begin{tabular}{lc} 
Comparisons & Number of genes in list \\
\hline Methylated in Sham but not AdjDBC or TumDBC & 271 \\
Methylated in AdjDBC but not Sham or TumDBC & 147 \\
Methylated in TumDBC but not Sham or AdjDBC & 150 \\
Methylated in Sham but not AdjBAP or TumBAP & 160 \\
Methylated in AdjBAP but not Sham or TumBAP & 159 \\
Methylated in TumBAP but not Sham or AdjBAP & 218 \\
Methylated in Sham but not TumDBC or TumBAP & 239 \\
Methylated in TumDBC but not Sham or TumBAP & 127 \\
Methylated in TumBAP but not Sham or TumDBC & 143 \\
Methylated in TumDBC and TumBAP but not Sham & 93 \\
\hline
\end{tabular}


Appendix E

Results of Gene Ontology Analyses for Differentially Methylated Genes 
TABLE E.1.

AgriGO GO Slim Results for Biological Process, Molecular Function and Cellular Compartment for Sham, AdjDBC and TumDBC Tissues

\begin{tabular}{|c|c|c|c|c|c|c|c|c|}
\hline \multirow[b]{2}{*}{ Ontology } & \multirow[b]{2}{*}{ GO Term } & \multirow[b]{2}{*}{ Description } & \multicolumn{2}{|c|}{$\begin{array}{l}\text { Methylated in } \\
\text { Sham tissues }\end{array}$} & \multicolumn{2}{|c|}{$\begin{array}{l}\text { Methylated in } \\
\text { AdjDBC tissue }\end{array}$} & \multicolumn{2}{|c|}{$\begin{array}{l}\text { Methylated in } \\
\text { TumDBC tissue }\end{array}$} \\
\hline & & & FDR & Num & FDR & Num & FDR & Num \\
\hline $\mathrm{P}$ & GO:0048856 & anatomical structure development & $5.00 \mathrm{E}-04$ & 39 & & & $1.50 \mathrm{E}-03$ & 25 \\
\hline $\mathrm{P}$ & GO:0009653 & anatomical structure morphogenesis & $2.10 \mathrm{E}-03$ & 24 & & & $3.20 \mathrm{E}-02$ & 13 \\
\hline $\mathrm{P}$ & GO:0065007 & biological regulation & $1.70 \mathrm{E}-03$ & 80 & & & $3.10 \mathrm{E}-03$ & 48 \\
\hline$P$ & GO:0009058 & biosynthetic process & $5.60 \mathrm{E}-03$ & 49 & & & $2.10 \mathrm{E}-03$ & 33 \\
\hline $\mathrm{P}$ & GO:0005975 & carbohydrate metabolic process & $2.80 \mathrm{E}-02$ & 10 & & & & \\
\hline $\mathrm{P}$ & GO:0007154 & cell communication & & & & & $2.10 \mathrm{E}-02$ & 13 \\
\hline $\mathrm{P}$ & GO:0007049 & cell cycle & $1.50 \mathrm{E}-02$ & 14 & & & & \\
\hline $\mathrm{P}$ & GO:0030154 & cell differentiation & $1.50 \mathrm{E}-02$ & 26 & & & 7.40E-03 & 18 \\
\hline $\mathrm{P}$ & GO:0008283 & cell proliferation & $3.90 \mathrm{E}-02$ & 14 & & & $8.90 \mathrm{E}-03$ & 11 \\
\hline$P$ & GO:0044249 & cellular biosynthetic process & $3.80 \mathrm{E}-03$ & 49 & & & $1.60 \mathrm{E}-03$ & 33 \\
\hline$P$ & GO:0016043 & cellular component organization & $5.80 \mathrm{E}-03$ & 32 & & & $1.70 \mathrm{E}-03$ & 23 \\
\hline $\mathrm{P}$ & GO:0048869 & cellular developmental process & $1.50 \mathrm{E}-02$ & 27 & & & $1.10 \mathrm{E}-02$ & 18 \\
\hline$P$ & GO:0034645 & cellular macromolecule biosynthetic process & $1.80 \mathrm{E}-02$ & 35 & & & $1.20 \mathrm{E}-02$ & 23 \\
\hline$P$ & GO:0044260 & cellular macromolecule metabolic process & $8.80 \mathrm{E}-05$ & 63 & & & $2.50 \mathrm{E}-03$ & 36 \\
\hline $\mathrm{P}$ & GO:0044237 & cellular metabolic process & $5.40 \mathrm{E}-06$ & 82 & $2.10 \mathrm{E}-02$ & 42 & $2.90 \mathrm{E}-06$ & 54 \\
\hline $\mathrm{P}$ & GO:0009987 & cellular process & $1.10 \mathrm{E}-06$ & 131 & $2.10 \mathrm{E}-02$ & 66 & 4.70E-09 & 87 \\
\hline $\mathrm{P}$ & GO:0044267 & cellular protein metabolic process & $1.30 \mathrm{E}-02$ & 28 & & & & \\
\hline $\mathrm{P}$ & GO:0032502 & developmental process & $1.20 \mathrm{E}-04$ & 47 & & & $2.70 \mathrm{E}-03$ & 27 \\
\hline $\mathrm{P}$ & GO:0051234 & establishment of localization & & & & & $2.70 \mathrm{E}-03$ & 24 \\
\hline $\mathrm{P}$ & GO:0010467 & gene expression & $5.60 \mathrm{E}-03$ & 40 & & & 4.20E-03 & 26 \\
\hline$P$ & GO:0040007 & growth & & & & & $3.50 \mathrm{E}-02$ & 7 \\
\hline $\mathrm{P}$ & GO:0006629 & lipid metabolic process & & & & & $1.00 \mathrm{E}-03$ & 13 \\
\hline $\mathrm{P}$ & GO:0051179 & localization & $6.20 \mathrm{E}-03$ & 39 & & & $2.30 \mathrm{E}-03$ & 27 \\
\hline$P$ & GO:0009059 & macromolecule biosynthetic process & $1.90 \mathrm{E}-02$ & 35 & & & $1.20 \mathrm{E}-02$ & 23 \\
\hline $\mathrm{P}$ & GO:0043170 & macromolecule metabolic process & 4.90E-06 & 73 & & & $2.50 \mathrm{E}-03$ & 39 \\
\hline $\mathrm{P}$ & GO:0043412 & macromolecule modification & $5.00 \mathrm{E}-04$ & 26 & & & & \\
\hline$P$ & GO:0008152 & metabolic process & $2.30 \mathrm{E}-07$ & 98 & $7.80 \mathrm{E}-03$ & 49 & $2.70 \mathrm{E}-05$ & 57 \\
\hline $\mathrm{P}$ & GO:0007275 & multicellular organismal development & $4.00 \mathrm{E}-05$ & 46 & & & $2.40 \mathrm{E}-03$ & 26 \\
\hline $\mathrm{P}$ & GO:0032501 & multicellular organismal process & $6.20 \mathrm{E}-03$ & 57 & $2.80 \mathrm{E}-03$ & 40 & $1.50 \mathrm{E}-02$ & 34 \\
\hline$P$ & GO:0006807 & $\begin{array}{l}\text { nitrogen compound metabolic process } \\
\text { nucleobase, nucleoside, nucleotide and nucleic acid }\end{array}$ & $3.60 \mathrm{E}-03$ & 46 & & & $2.50 \mathrm{E}-02$ & 26 \\
\hline $\mathrm{P}$ & GO:0006139 & metabolic process & $6.70 \mathrm{E}-03$ & 41 & & & $8.60 \mathrm{E}-03$ & 26 \\
\hline
\end{tabular}




\begin{tabular}{|c|c|c|c|c|c|c|c|c|}
\hline \multirow[b]{2}{*}{ Ontology } & \multirow[b]{2}{*}{ GO Term } & \multirow[b]{2}{*}{ Description } & \multicolumn{2}{|c|}{$\begin{array}{l}\text { Methylated in } \\
\text { Sham tissues }\end{array}$} & \multicolumn{2}{|c|}{$\begin{array}{l}\text { Methylated in } \\
\text { AdjDBC tissue }\end{array}$} & \multicolumn{2}{|c|}{$\begin{array}{l}\text { Methylated in } \\
\text { TumDBC tissue }\end{array}$} \\
\hline & & & FDR & Num & FDR & Num & FDR & Num \\
\hline $\mathrm{P}$ & GO:0006996 & organelle organization & $1.80 \mathrm{E}-02$ & 19 & & & & \\
\hline $\mathrm{P}$ & GO:0044238 & primary metabolic process & $1.80 \mathrm{E}-06$ & 85 & & & $4.50 \mathrm{E}-05$ & 51 \\
\hline $\mathrm{P}$ & GO:0019538 & protein metabolic process & $2.70 \mathrm{E}-03$ & 36 & & & & \\
\hline$P$ & GO:0006464 & protein modification process & $6.60 \mathrm{E}-04$ & 25 & & & & \\
\hline $\mathrm{P}$ & GO:0050789 & regulation of biological process & $9.40 \mathrm{E}-03$ & 69 & & & $3.10 \mathrm{E}-02$ & 40 \\
\hline $\mathrm{P}$ & GO:0065008 & regulation of biological quality & $5.60 \mathrm{E}-03$ & 29 & & & $2.70 \mathrm{E}-03$ & 20 \\
\hline $\mathrm{P}$ & GO:0050794 & regulation of cellular process & $2.00 \mathrm{E}-02$ & 64 & & & $3.50 \mathrm{E}-02$ & 38 \\
\hline $\mathrm{P}$ & GO:0010468 & regulation of gene expression & $5.00 \mathrm{E}-04$ & 36 & & & & \\
\hline $\mathrm{P}$ & GO:0060255 & regulation of macromolecule metabolic process & $2.00 \mathrm{E}-03$ & 37 & & & $8.90 \mathrm{E}-03$ & 22 \\
\hline $\mathrm{P}$ & GO:0019222 & regulation of metabolic process & $5.60 \mathrm{E}-03$ & 38 & & & $1.50 \mathrm{E}-03$ & 27 \\
\hline $\mathrm{P}$ & GO:0000003 & reproduction & $5.80 \mathrm{E}-03$ & 14 & & & $1.40 \mathrm{E}-02$ & 9 \\
\hline $\mathrm{P}$ & GO:0006350 & transcription & $5.80 \mathrm{E}-03$ & 30 & & & & \\
\hline $\mathrm{P}$ & GO:0006810 & transport & 4.90E-02 & 31 & & & $2.70 \mathrm{E}-03$ & 24 \\
\hline $\mathrm{F}$ & GO:0005488 & binding & $1.50 \mathrm{E}-17$ & 152 & $1.20 \mathrm{E}-03$ & 66 & 4.70E-09 & 83 \\
\hline $\mathrm{F}$ & GO:0003824 & catalytic activity & 4.30E-08 & 76 & $7.60 \mathrm{E}-03$ & 36 & $2.20 \mathrm{E}-05$ & 45 \\
\hline $\mathrm{F}$ & GO:0043169 & cation binding & $4.30 \mathrm{E}-08$ & 58 & & & $4.00 \mathrm{E}-02$ & 24 \\
\hline $\mathrm{F}$ & GO:0043167 & ion binding & 4.30E-08 & 58 & & & $2.70 \mathrm{E}-02$ & 25 \\
\hline $\mathrm{F}$ & GO:0046872 & metal ion binding & 4.30E-08 & 58 & & & 3.90E-02 & 24 \\
\hline $\mathrm{F}$ & GO:0005515 & protein binding & $1.30 \mathrm{E}-05$ & 73 & 8.40E-04 & 42 & $6.90 \mathrm{E}-03$ & 40 \\
\hline $\mathrm{F}$ & GO:0003677 & DNA binding & $1.00 \mathrm{E}-04$ & 32 & & & & \\
\hline $\mathrm{F}$ & GO:0005509 & calcium ion binding & $1.00 \mathrm{E}-04$ & 15 & & & & \\
\hline $\mathrm{F}$ & GO:0016787 & hydrolase activity & $1.70 \mathrm{E}-04$ & 36 & & & $8.10 \mathrm{E}-03$ & 21 \\
\hline $\mathrm{F}$ & GO:0003676 & nucleic acid binding & $1.90 \mathrm{E}-03$ & 37 & & & $2.00 \mathrm{E}-02$ & 22 \\
\hline $\mathrm{F}$ & GO:0000166 & nucleotide binding & $5.00 \mathrm{E}-03$ & 31 & & & $6.90 \mathrm{E}-03$ & 21 \\
\hline $\mathrm{F}$ & GO:0016773 & phosphotransferase activity, alcohol group as acceptor & $1.00 \mathrm{E}-02$ & 14 & & & & \\
\hline $\mathrm{F}$ & GO:0016301 & kinase activity & $4.10 \mathrm{E}-02$ & 14 & & & & \\
\hline $\mathrm{C}$ & GO:0044464 & cell part & $8.70 \mathrm{E}-21$ & 190 & $1.50 \mathrm{E}-08$ & 98 & $3.20 \mathrm{E}-13$ & 108 \\
\hline $\mathrm{C}$ & GO:0005623 & cell & $8.70 \mathrm{E}-21$ & 190 & $1.50 \mathrm{E}-08$ & 98 & 3.20E-13 & 108 \\
\hline $\mathrm{C}$ & GO:0005622 & intracellular & $2.90 \mathrm{E}-15$ & 141 & & & $3.10 \mathrm{E}-08$ & 77 \\
\hline $\mathrm{C}$ & GO:0044424 & intracellular part & $5.30 \mathrm{E}-15$ & 138 & & & $3.50 \mathrm{E}-08$ & 75 \\
\hline $\mathrm{C}$ & GO:0043229 & intracellular organelle & $8.00 \mathrm{E}-12$ & 117 & & & 3.10E-08 & 68 \\
\hline $\mathrm{C}$ & GO:0043226 & organelle & $8.30 \mathrm{E}-12$ & 117 & & & 3.10E-08 & 68 \\
\hline $\mathrm{C}$ & GO:0043231 & intracellular membrane-bounded organelle & $9.60 \mathrm{E}-11$ & 105 & & & 3.10E-08 & 63 \\
\hline $\mathrm{C}$ & GO:0043227 & membrane-bounded organelle & $9.60 \mathrm{E}-11$ & 105 & & & $3.10 \mathrm{E}-08$ & 63 \\
\hline
\end{tabular}




\begin{tabular}{|c|c|c|c|c|c|c|c|c|}
\hline \multirow[b]{2}{*}{ Ontology } & \multirow[b]{2}{*}{ GO Term } & \multirow[b]{2}{*}{ Description } & \multicolumn{2}{|c|}{$\begin{array}{l}\text { Methylated in } \\
\text { Sham tissues }\end{array}$} & \multicolumn{2}{|c|}{$\begin{array}{l}\text { Methylated in } \\
\text { AdjDBC tissue }\end{array}$} & \multicolumn{2}{|c|}{$\begin{array}{l}\text { Methylated in } \\
\text { TumDBC tissue }\end{array}$} \\
\hline & & & FDR & Num & FDR & Num & FDR & Num \\
\hline $\mathrm{C}$ & GO:0005737 & cytoplasm & $8.40 \mathrm{E}-10$ & 98 & & & $6.30 \mathrm{E}-05$ & 52 \\
\hline $\mathrm{C}$ & GO:0044444 & cytoplasmic part & 5.60E-08 & 67 & & & $5.70 \mathrm{E}-04$ & 35 \\
\hline $\mathrm{C}$ & GO:0016020 & membrane & $5.00 \mathrm{E}-05$ & 90 & $5.20 \mathrm{E}-08$ & 64 & 4.40E-06 & 59 \\
\hline $\mathrm{C}$ & GO:0005634 & nucleus & $5.80 \mathrm{E}-05$ & 59 & & & $3.50 \mathrm{E}-04$ & 36 \\
\hline $\mathrm{C}$ & GO:0042995 & cell projection & $4.90 \mathrm{E}-04$ & 19 & & & & \\
\hline $\mathrm{C}$ & GO:0005654 & nucleoplasm & $1.60 \mathrm{E}-03$ & 12 & & & & \\
\hline $\mathrm{C}$ & GO:0005783 & endoplasmic reticulum & $1.90 \mathrm{E}-03$ & 18 & & & & \\
\hline $\mathrm{C}$ & GO:0031974 & membrane-enclosed lumen & 4.30E-03 & 15 & & & & \\
\hline $\mathrm{C}$ & GO:0070013 & intracellular organelle lumen & 7.20E-03 & 14 & & & & \\
\hline $\mathrm{C}$ & GO:0043233 & organelle lumen & 7.20E-03 & 14 & & & & \\
\hline $\mathrm{C}$ & GO:0044446 & intracellular organelle part & 7.70E-03 & 36 & & & 7.70E-03 & 23 \\
\hline $\mathrm{C}$ & GO:0044422 & organelle part & $8.80 \mathrm{E}-03$ & 36 & & & $8.40 \mathrm{E}-03$ & 23 \\
\hline $\mathrm{C}$ & GO:0005576 & extracellular region & 1.10E-02 & 25 & $2.20 \mathrm{E}-02$ & 17 & $9.60 \mathrm{E}-04$ & 19 \\
\hline $\mathrm{C}$ & GO:0031981 & nuclear lumen & $1.10 \mathrm{E}-02$ & 12 & & & & \\
\hline $\mathrm{C}$ & GO:0005773 & vacuole & $1.50 \mathrm{E}-02$ & 7 & & & & \\
\hline $\mathrm{C}$ & GO:0005886 & plasma membrane & $1.60 \mathrm{E}-02$ & 37 & & & $1.90 \mathrm{E}-02$ & 23 \\
\hline $\mathrm{C}$ & GO:0043234 & protein complex & $2.80 \mathrm{E}-02$ & 27 & & & & \\
\hline $\mathrm{C}$ & GO:0044428 & nuclear part & 4.20E-02 & 15 & & & & \\
\hline $\mathrm{P}$ & GO:0033036 & macromolecule localization & $3.40 \mathrm{E}-02$ & 16 & & & & \\
\hline $\mathrm{P}$ & GO:0008104 & protein localization & 4.10E-02 & 14 & & & & \\
\hline $\mathrm{F}$ & GO:0004871 & signal transducer activity & & & 8.40E-04 & 27 & & \\
\hline $\mathrm{F}$ & GO:0004872 & receptor activity & & & $8.40 \mathrm{E}-04$ & 26 & & \\
\hline $\mathrm{F}$ & GO:0060089 & molecular transducer activity & & & 8.40E-04 & 27 & & \\
\hline $\mathrm{F}$ & GO:0005102 & receptor binding & & & $1.40 \mathrm{E}-02$ & 10 & & \\
\hline $\mathrm{P}$ & GO:0006091 & generation of precursor metabolites and energy & 4.10E-02 & 7 & & & & \\
\hline $\mathrm{P}$ & GO:0007010 & cytoskeleton organization & 4.90E-02 & 8 & & & & \\
\hline $\mathrm{P}$ & GO:0008219 & cell death & & & $6.40 \mathrm{E}-03$ & 14 & & \\
\hline $\mathrm{P}$ & GO:0016265 & death & & & $6.40 \mathrm{E}-03$ & 14 & & \\
\hline $\mathrm{F}$ & GO:0003723 & RNA binding & & & & & $8.50 \mathrm{E}-03$ & 10 \\
\hline $\mathrm{F}$ & GO:0005215 & transporter activity & & & & & $2.30 \mathrm{E}-02$ & 12 \\
\hline $\mathrm{F}$ & GO:0016740 & transferase activity & & & & & $2.50 \mathrm{E}-02$ & 16 \\
\hline $\mathrm{F}$ & GO:0015075 & ion transmembrane transporter activity & & & & & $2.50 \mathrm{E}-02$ & 9 \\
\hline $\mathrm{F}$ & GO:0030234 & enzyme regulator activity & & & & & 2.70E-02 & 9 \\
\hline $\mathrm{F}$ & GO:0022857 & transmembrane transporter activity & & & & & 2.70E-02 & 10 \\
\hline
\end{tabular}




\begin{tabular}{|c|c|c|c|c|c|c|c|c|}
\hline \multirow[b]{2}{*}{ Ontology } & \multirow[b]{2}{*}{ GO Term } & \multirow[b]{2}{*}{ Description } & \multicolumn{2}{|c|}{$\begin{array}{l}\text { Methylated in } \\
\text { Sham tissues }\end{array}$} & \multicolumn{2}{|c|}{$\begin{array}{l}\text { Methylated in } \\
\text { AdjDBC tissue }\end{array}$} & \multicolumn{2}{|c|}{$\begin{array}{l}\text { Methylated in } \\
\text { TumDBC tissue }\end{array}$} \\
\hline & & & FDR & Num & FDR & Num & FDR & Num \\
\hline $\mathrm{F}$ & GO:0022892 & substrate-specific transporter activity & & & & & $3.10 \mathrm{E}-02$ & 10 \\
\hline $\mathrm{F}$ & GO:0022891 & substrate-specific transmembrane transporter activity & & & & & $3.80 \mathrm{E}-02$ & 9 \\
\hline $\mathrm{F}$ & GO:0022803 & passive transmembrane transporter activity & & & & & 4.00E-02 & 6 \\
\hline $\mathrm{F}$ & GO:0015267 & channel activity & & & & & 4.00E-02 & 6 \\
\hline $\mathrm{C}$ & GO:0005794 & Golgi apparatus & & & & & $2.70 \mathrm{E}-03$ & 11 \\
\hline $\mathrm{C}$ & GO:0005739 & mitochondrion & & & & & $2.70 \mathrm{E}-03$ & 16 \\
\hline $\mathrm{C}$ & GO:0044421 & extracellular region part & & & & & $9.40 \mathrm{E}-03$ & 11 \\
\hline $\mathrm{C}$ & GO:0005578 & proteinaceous extracellular matrix & & & & & $9.80 \mathrm{E}-03$ & 6 \\
\hline $\mathrm{C}$ & GO:0031012 & extracellular matrix & & & & & $1.50 \mathrm{E}-02$ & 6 \\
\hline $\mathrm{C}$ & GO:0031967 & organelle envelope & & & & & 4.10E-02 & 7 \\
\hline $\mathrm{C}$ & GO:0031975 & envelope & & & & & $4.30 \mathrm{E}-02$ & 7 \\
\hline
\end{tabular}

Note: Gene lists for functional ontology analysis were generated by selecting features with $\log _{2}$ peak ratio $>2$ (indicates methylated DNA) in all three samples of interes and excluding all features that were not methylated $\left(\log _{2}\right.$ peak ratio $\left.<2\right)$ in at least 2 of the 3 biological samples not of interest. Thus, genes in the methylated Sham list were consistently methylated in only the sham tissues. Gene ontology was performed using AgrGo (Du, et al., 2010) using the singular entichment analysis tool against the mouse the minimum numer of mopping entries set at 5 genes. The gene ontology type performed was a generic GO slim (Gene Ontology Consortium). GO terms shown abo a the minimum number of mapping entries set at 5 genes. The gene ontology type performed was a generic GO slim (Gene Ontology Consortium). GO terms shown above are for are shown. Color blocks indicate the level of significance for each GO term, color-coded according to the tissue type for cross reference with figures and supplemental materist are shown. Color blocks indicate the level of significance for each GO term, color-coded according to the tissue type for cross reference with figures and supplemental materials (gray scale, Shan, blie scale, AdDBC, red scale, TunDBC). Absence of $P$ value and gene numbers for a GO term indicates that hat tern was not significanty enriched for that particular gene set. Fr clatily, in cases wherea bion ifferent for each tissue. 
TABLE E.2.

Agrigo GO Slim Results for Biological Process, Molecular Function and Cellular Compartment for Sham, AdjBaP and TumBaP Tissues

\begin{tabular}{|c|c|c|c|c|c|c|c|c|}
\hline \multirow[b]{2}{*}{ Ontology } & \multirow[b]{2}{*}{ GO Term } & \multirow[b]{2}{*}{ Description } & \multicolumn{2}{|c|}{$\begin{array}{l}\text { Methylated in Sham } \\
\text { tissues }\end{array}$} & \multicolumn{2}{|c|}{$\begin{array}{l}\text { Methylated in } \\
\text { AdjBaP tissue }\end{array}$} & \multicolumn{2}{|c|}{$\begin{array}{l}\text { Methylated in } \\
\text { TumBaP tissue }\end{array}$} \\
\hline & & & FDR & Num & FDR & Num & FDR & Num \\
\hline $\mathrm{P}$ & GO:0048856 & anatomical structure development & $7.50 \mathrm{E}-03$ & 26 & & & $2.60 \mathrm{E}-06$ & 40 \\
\hline $\mathrm{P}$ & GO:0009653 & anatomical structure morphogenesis & $5.10 \mathrm{E}-05$ & 22 & & & $5.00 \mathrm{E}-05$ & 25 \\
\hline $\mathrm{P}$ & GO:0065007 & biological regulation & & & & & $2.40 \mathrm{E}-04$ & 70 \\
\hline $\mathrm{P}$ & GO:0009058 & biosynthetic process & & & $5.50 \mathrm{E}-04$ & 36 & $5.30 \mathrm{E}-05$ & 49 \\
\hline $\mathrm{P}$ & GO:0005975 & carbohydrate metabolic process & & & $2.10 \mathrm{E}-02$ & 8 & $2.30 \mathrm{E}-02$ & 9 \\
\hline $\mathrm{P}$ & GO:0007154 & cell communication & & & & & $2.40 \mathrm{E}-04$ & 22 \\
\hline $\mathrm{P}$ & GO:0030154 & cell differentiation & & & $3.80 \mathrm{E}-02$ & 17 & $2.20 \mathrm{E}-03$ & 25 \\
\hline$P$ & GO:0008283 & cell proliferation & $3.90 \mathrm{E}-02$ & 12 & & & $2.00 \mathrm{E}-02$ & 13 \\
\hline $\mathrm{P}$ & GO:0007267 & cell-cell signaling & & & & & $1.10 \mathrm{E}-02$ & 10 \\
\hline $\mathrm{P}$ & GO:0006519 & cellular amino acid and derivative metabolic process & & & $2.80 \mathrm{E}-03$ & 8 & & \\
\hline $\mathrm{P}$ & GO:0044249 & cellular biosynthetic process & & & $3.60 \mathrm{E}-04$ & 36 & 8.40E-05 & 47 \\
\hline $\mathrm{P}$ & GO:0048869 & cellular developmental process & & & & & 4.00E-03 & 25 \\
\hline $\mathrm{P}$ & GO:0034645 & cellular macromolecule biosynthetic process & & & $2.10 \mathrm{E}-02$ & 24 & $6.20 \mathrm{E}-04$ & 35 \\
\hline $\mathrm{P}$ & GO:0044260 & cellular macromolecule metabolic process & & & & & $6.20 \mathrm{E}-04$ & 50 \\
\hline $\mathrm{P}$ & GO:0044237 & cellular metabolic process & & & $3.10 \mathrm{E}-05$ & 53 & $8.40 \mathrm{E}-05$ & 65 \\
\hline $\mathrm{P}$ & GO:0009987 & cellular process & & & 4.90E-05 & 79 & $1.00 \mathrm{E}-09$ & 119 \\
\hline $\mathrm{P}$ & GO:0044267 & cellular protein metabolic process & & & & & $1.30 \mathrm{E}-02$ & 24 \\
\hline $\mathrm{P}$ & GO:0032502 & developmental process & $3.90 \mathrm{E}-02$ & 27 & & & $6.00 \mathrm{E}-05$ & 41 \\
\hline$P$ & GO:0051234 & establishment of localization & & & $2.00 \mathrm{E}-02$ & 23 & $8.40 \mathrm{E}-05$ & 36 \\
\hline $\mathrm{P}$ & GO:0045184 & establishment of protein localization & & & $2.50 \mathrm{E}-02$ & 10 & & \\
\hline $\mathrm{P}$ & GO:0042592 & homeostatic process & & & & & $2.20 \mathrm{E}-02$ & 12 \\
\hline$P$ & GO:0006629 & lipid metabolic process & & & $3.30 \mathrm{E}-04$ & 14 & $2.30 \mathrm{E}-02$ & 12 \\
\hline $\mathrm{P}$ & GO:0051179 & localization & & & $3.70 \mathrm{E}-02$ & 24 & 4.90E-05 & 41 \\
\hline $\mathrm{P}$ & GO:0009059 & macromolecule biosynthetic process & & & $2.10 \mathrm{E}-02$ & 24 & $1.50 \mathrm{E}-04$ & 37 \\
\hline $\mathrm{P}$ & GO:0033036 & macromolecule localization & & & $2.50 \mathrm{E}-02$ & 12 & & \\
\hline $\mathrm{P}$ & GO:0043170 & macromolecule metabolic process & & & & & $5.10 \mathrm{E}-05$ & 59 \\
\hline $\mathrm{P}$ & GO:0008152 & metabolic process & $3.90 \mathrm{E}-02$ & 50 & $3.10 \mathrm{E}-05$ & 59 & 4.60E-07 & 82 \\
\hline $\mathrm{P}$ & GO:0007275 & multicellular organismal development & 3.90E-02 & 25 & & & 5.50E-05 & 39 \\
\hline $\mathrm{P}$ & GO:0032501 & multicellular organismal process & & & $2.30 \mathrm{E}-04$ & 43 & $2.30 \mathrm{E}-03$ & 50 \\
\hline $\mathrm{P}$ & GO:0006807 & nitrogen compound metabolic process & & & $8.20 \mathrm{E}-03$ & 30 & $1.70 \mathrm{E}-02$ & 36 \\
\hline $\mathrm{P}$ & GO:0044238 & primary metabolic process & & & $2.40 \mathrm{E}-05$ & 55 & $1.10 \mathrm{E}-05$ & 70 \\
\hline $\mathrm{P}$ & GO:0019538 & protein metabolic process & & & & & $3.70 \mathrm{E}-03$ & 30 \\
\hline
\end{tabular}




\begin{tabular}{|c|c|c|c|c|c|c|c|c|}
\hline \multirow[b]{2}{*}{ Ontology } & \multirow[b]{2}{*}{ GO Term } & \multirow[b]{2}{*}{ Description } & \multicolumn{2}{|c|}{$\begin{array}{l}\text { Methylated in Sham } \\
\text { tissues }\end{array}$} & \multicolumn{2}{|c|}{$\begin{array}{l}\text { Methylated in } \\
\text { AdjBaP tissue }\end{array}$} & \multicolumn{2}{|c|}{$\begin{array}{l}\text { Methylated in } \\
\text { TumBaP tissue }\end{array}$} \\
\hline & & & FDR & Num & FDR & Num & FDR & Num \\
\hline $\mathrm{P}$ & GO:0050789 & regulation of biological process & & & & & $1.20 \mathrm{E}-03$ & 62 \\
\hline$P$ & GO:0065008 & regulation of biological quality & & & & & $1.70 \mathrm{E}-02$ & 23 \\
\hline$P$ & GO:0050794 & regulation of cellular process & & & & & $2.90 \mathrm{E}-03$ & 58 \\
\hline$P$ & GO:0060255 & regulation of macromolecule metabolic process & & & & & $9.10 \mathrm{E}-03$ & 29 \\
\hline$P$ & GO:0019222 & regulation of metabolic process & & & & & $6.60 \mathrm{E}-03$ & 32 \\
\hline$P$ & GO:0050896 & response to stimulus & & & 4.00E-02 & 22 & & \\
\hline $\mathrm{P}$ & GO:0006412 & translation & & & & & $8.00 \mathrm{E}-03$ & 10 \\
\hline $\mathrm{P}$ & GO:0006810 & transport & & & $2.00 \mathrm{E}-02$ & 23 & $8.40 \mathrm{E}-05$ & 36 \\
\hline $\mathrm{F}$ & GO:0005488 & binding & $3.30 \mathrm{E}-05$ & 78 & $4.60 \mathrm{E}-06$ & 78 & $1.70 \mathrm{E}-10$ & 115 \\
\hline $\mathrm{F}$ & GO:0043169 & cation binding & $3.00 \mathrm{E}-03$ & 30 & $1.10 \mathrm{E}-03$ & 30 & & \\
\hline $\mathrm{F}$ & GO:0043167 & ion binding & $3.00 \mathrm{E}-03$ & 30 & $1.10 \mathrm{E}-03$ & 30 & & \\
\hline $\mathrm{F}$ & GO:0046872 & metal ion binding & $3.00 \mathrm{E}-03$ & 30 & $1.10 \mathrm{E}-03$ & 30 & & \\
\hline $\mathrm{F}$ & GO:0003677 & DNA binding & $4.00 \mathrm{E}-02$ & 17 & & & & \\
\hline $\mathrm{F}$ & GO:0016788 & hydrolase activity, acting on ester bonds & $5.00 \mathrm{E}-02$ & 9 & & & & \\
\hline $\mathrm{F}$ & GO:0003824 & catalytic activity & & & $2.70 \mathrm{E}-06$ & 49 & $2.20 \mathrm{E}-04$ & 56 \\
\hline $\mathrm{F}$ & GO:0060089 & molecular transducer activity & & & $1.60 \mathrm{E}-04$ & 30 & & \\
\hline $\mathrm{F}$ & GO:0004871 & signal transducer activity & & & $1.60 \mathrm{E}-04$ & 30 & & \\
\hline $\mathrm{F}$ & GO:0004872 & receptor activity & & & $2.10 \mathrm{E}-04$ & 28 & & \\
\hline $\mathrm{F}$ & GO:0016787 & hydrolase activity & & & $5.00 \mathrm{E}-04$ & 24 & & \\
\hline $\mathrm{F}$ & GO:0008289 & lipid binding & & & $3.70 \mathrm{E}-02$ & 6 & & \\
\hline $\mathrm{F}$ & GO:0005515 & protein binding & & & & & 4.30E-05 & 62 \\
\hline $\mathrm{F}$ & GO:0005215 & transporter activity & & & & & $2.60 \mathrm{E}-03$ & 18 \\
\hline $\mathrm{C}$ & GO:0044464 & cell part & $2.80 \mathrm{E}-09$ & 107 & $5.80 \mathrm{E}-11$ & 108 & $7.00 \mathrm{E}-27$ & 170 \\
\hline $\mathrm{C}$ & GO:0005623 & cell & $2.80 \mathrm{E}-09$ & 107 & $5.80 \mathrm{E}-11$ & 108 & 7.00E-27 & 170 \\
\hline $\mathrm{C}$ & GO:0005622 & intracellular & $5.60 \mathrm{E}-05$ & 73 & $1.60 \mathrm{E}-04$ & 70 & $3.20 \mathrm{E}-17$ & 124 \\
\hline $\mathrm{C}$ & GO:0044424 & intracellular part & $5.60 \mathrm{E}-05$ & 72 & $1.60 \mathrm{E}-04$ & 69 & $1.20 \mathrm{E}-15$ & 119 \\
\hline $\mathrm{C}$ & GO:0043229 & intracellular organelle & $4.10 \mathrm{E}-04$ & 61 & $2.50 \mathrm{E}-03$ & 57 & $6.70 \mathrm{E}-10$ & 95 \\
\hline $\mathrm{C}$ & GO:0043226 & organelle & $4.10 \mathrm{E}-04$ & 61 & $2.50 \mathrm{E}-03$ & 57 & $6.70 \mathrm{E}-10$ & 95 \\
\hline $\mathrm{C}$ & GO:0005576 & extracellular region & $1.50 \mathrm{E}-03$ & 20 & & & $1.00 \mathrm{E}-02$ & 22 \\
\hline $\mathrm{C}$ & GO:0016020 & membrane & $3.00 \mathrm{E}-03$ & 54 & $2.10 \mathrm{E}-09$ & 70 & $1.60 \mathrm{E}-12$ & 96 \\
\hline $\mathrm{C}$ & GO:0043231 & intracellular membrane-bounded organelle & $1.20 \mathrm{E}-02$ & 50 & $3.60 \mathrm{E}-03$ & 51 & 4.40E-07 & 80 \\
\hline $\mathrm{C}$ & GO:0043227 & membrane-bounded organelle & $1.20 \mathrm{E}-02$ & 50 & $3.60 \mathrm{E}-03$ & 51 & 4.40E-07 & 80 \\
\hline $\mathrm{C}$ & GO:0005737 & cytoplasm & $2.60 \mathrm{E}-02$ & 46 & $3.30 \mathrm{E}-03$ & 49 & $8.30 \mathrm{E}-14$ & 93 \\
\hline $\mathrm{C}$ & GO:0044444 & cytoplasmic part & 4.00E-02 & 31 & $1.60 \mathrm{E}-03$ & 36 & $6.70 \mathrm{E}-10$ & 62 \\
\hline
\end{tabular}




\begin{tabular}{|c|c|c|c|c|c|c|c|c|}
\hline \multirow[b]{2}{*}{ Ontology } & \multirow[b]{2}{*}{ GO Term } & \multirow[b]{2}{*}{ Description } & \multicolumn{2}{|c|}{$\begin{array}{l}\text { Methylated in Sham } \\
\text { tissues }\end{array}$} & \multicolumn{2}{|c|}{$\begin{array}{l}\text { Methylated in } \\
\text { AdjBaP tissue }\end{array}$} & \multicolumn{2}{|c|}{$\begin{array}{l}\text { Methylated in } \\
\text { TumBaP tissue }\end{array}$} \\
\hline & & & FDR & Num & FDR & Num & FDR & Num \\
\hline $\mathrm{C}$ & GO:0005886 & plasma membrane & & & 7.10E-03 & 26 & $2.90 \mathrm{E}-08$ & 47 \\
\hline $\mathrm{C}$ & GO:0005794 & Golgi apparatus & & & $2.00 \mathrm{E}-02$ & 10 & & \\
\hline $\mathrm{C}$ & GO:0005768 & endosome & & & $2.80 \mathrm{E}-02$ & 6 & & \\
\hline $\mathrm{C}$ & GO:0005773 & vacuole & & & $3.50 \mathrm{E}-02$ & 5 & & \\
\hline $\mathrm{C}$ & GO:0005829 & cytosol & & & & & $6.00 \mathrm{E}-04$ & 13 \\
\hline $\mathrm{C}$ & GO:0005739 & mitochondrion & & & & & $1.40 \mathrm{E}-02$ & 19 \\
\hline $\mathrm{C}$ & GO:0044422 & organelle part & & & & & $1.60 \mathrm{E}-02$ & 30 \\
\hline $\mathrm{C}$ & GO:0016023 & cytoplasmic membrane-bounded vesicle & & & & & $2.30 \mathrm{E}-02$ & 8 \\
\hline $\mathrm{C}$ & GO:0044446 & intracellular organelle part & & & & & $2.40 \mathrm{E}-02$ & 29 \\
\hline $\mathrm{C}$ & GO:0031988 & membrane-bounded vesicle & & & & & $3.10 \mathrm{E}-02$ & 8 \\
\hline $\mathrm{C}$ & GO:0005634 & nucleus & & & & & $3.70 \mathrm{E}-02$ & 40 \\
\hline $\mathrm{C}$ & GO:0043232 & intracellular non-membrane-bounded organelle & & & & & $3.80 \mathrm{E}-02$ & 21 \\
\hline $\mathrm{C}$ & GO:0043228 & non-membrane-bounded organelle & & & & & $3.80 \mathrm{E}-02$ & 21 \\
\hline
\end{tabular}

interest and excluding all features that were not methylated ( $\log _{2}$ peak ratio $<2$ ) in at least 2 of the 3 biological samples not of interest. Thus, genes in the methylated Sham list were consistently methylated in only the sham tissues. Gene ontology was performed using AgriGO (Du, et al., 2010) using the singular enrichment analysis tool against the mouse gene ontology database (Mouse Genome Informatics) with the following parameters: Fisher test with FDR under dependency correction and significance level of $P<0.05$ and the minimum number of mapping entries set at 5 genes. The gene ontology type performed was a generic GO slim (Gene Ontology Consortium). GO terms shown above are for biological processes (B), molecular function (F) and cellular component (C). For each tissue, the FDR-adjusted $P$ value and the number of genes associated with the GO term are shown. Color blocks indicate the level of significance for each GO term, color-coded according to the tissue type for cross reference with Figures and supplemental materials (gray scale, Sham; green scale, AdjBAP; orange scale, TumBAP). Absence of $P$ value and gene numbers for a $\mathrm{GO}$ term indicates that that term was not significantly enriched for that particular gene set. For clarity, in cases where a biological process is represented in more than one tissue type, the specific methylated genes associated with that term are different for each tissue. 
TABLE E.3.

Agrigo GO Slim Results for Biological Process, Molecular Function and Cellular Compartment for Sham, TumDBC and TumBaP Tissues

\begin{tabular}{|c|c|c|c|c|c|c|c|c|c|c|}
\hline \multirow[b]{2}{*}{ Ontology } & \multirow[b]{2}{*}{ GO Term } & \multirow[b]{2}{*}{ Description } & \multicolumn{2}{|c|}{$\begin{array}{l}\text { Methylated in Sham } \\
\text { tissues }\end{array}$} & \multicolumn{2}{|c|}{$\begin{array}{l}\text { Methylated in } \\
\text { TumDBC tissue }\end{array}$} & \multicolumn{2}{|c|}{$\begin{array}{l}\text { Methylated in } \\
\text { TumBaP tissue }\end{array}$} & \multicolumn{2}{|c|}{$\begin{array}{c}\text { Methylated in } \\
\text { TumDBC+TumBaP } \\
\text { tissues }\end{array}$} \\
\hline & & & FDR & Num & FDR & Num & FDR & Num & FDR & Num \\
\hline$P$ & GO:0048856 & anatomical structure development & $2.10 \mathrm{E}-05$ & 40 & & & $1.20 \mathrm{E}-02$ & 22 & $1.30 \mathrm{E}-03$ & 19 \\
\hline $\mathrm{P}$ & GO:0009653 & anatomical structure morphogenesis & $1.20 \mathrm{E}-05$ & 28 & & & $3.60 \mathrm{E}-03$ & 16 & $6.80 \mathrm{E}-03$ & 11 \\
\hline$P$ & GO:0065007 & biological regulation & $1.90 \mathrm{E}-03$ & 72 & & & 3.70E-02 & 43 & & \\
\hline $\mathrm{P}$ & GO:0009058 & biosynthetic process & 7.10E-03 & 44 & & & & & $1.40 \mathrm{E}-03$ & 24 \\
\hline$P$ & GO:0007154 & cell communication & & & & & $5.60 \mathrm{E}-03$ & 15 & & \\
\hline $\mathrm{P}$ & GO:0030154 & cell differentiation & $3.60 \mathrm{E}-03$ & 26 & & & $2.00 \mathrm{E}-02$ & 17 & & \\
\hline $\mathrm{P}$ & GO:0008283 & cell proliferation & $1.80 \mathrm{E}-02$ & 14 & & & & & & \\
\hline $\mathrm{P}$ & GO:0044249 & cellular biosynthetic process & $4.70 \mathrm{E}-03$ & 44 & & & & & $1.30 \mathrm{E}-03$ & 24 \\
\hline $\mathrm{P}$ & GO:0016043 & cellular component organization & $1.10 \mathrm{E}-03$ & 32 & & & & & & \\
\hline $\mathrm{P}$ & GO:0048869 & cellular developmental process & $1.90 \mathrm{E}-03$ & 28 & & & $2.90 \mathrm{E}-02$ & 17 & & \\
\hline $\mathrm{P}$ & GO:0034645 & cellular macromolecule biosynthetic process & $1.90 \mathrm{E}-03$ & 36 & & & & & $1.40 \mathrm{E}-03$ & 19 \\
\hline $\mathrm{P}$ & GO:0044260 & cellular macromolecule metabolic process & $3.90 \mathrm{E}-04$ & 55 & & & & & $2.70 \mathrm{E}-03$ & 25 \\
\hline $\mathrm{P}$ & GO:0044237 & cellular metabolic process & $2.10 \mathrm{E}-04$ & 69 & $4.90 \mathrm{E}-04$ & 42 & $2.00 \mathrm{E}-02$ & 40 & $1.30 \mathrm{E}-03$ & 32 \\
\hline $\mathrm{P}$ & GO:0009987 & cellular process & 4.70E-08 & 124 & $5.20 \mathrm{E}-05$ & 67 & $1.40 \mathrm{E}-05$ & 77 & $1.30 \mathrm{E}-03$ & 48 \\
\hline $\mathrm{P}$ & GO:0044267 & cellular protein metabolic process & $3.50 \mathrm{E}-02$ & 24 & & & & & & \\
\hline $\mathrm{P}$ & GO:0007010 & cytoskeleton organization & $3.00 \mathrm{E}-02$ & 8 & & & & & & \\
\hline $\mathrm{P}$ & GO:0032502 & developmental process & $3.50 \mathrm{E}-05$ & 45 & & & $2.20 \mathrm{E}-02$ & 24 & $1.30 \mathrm{E}-03$ & 21 \\
\hline $\mathrm{P}$ & GO:0009790 & embryonic development & $3.50 \mathrm{E}-02$ & 12 & & & & & & \\
\hline $\mathrm{P}$ & GO:0051234 & establishment of localization & & & & & $2.10 \mathrm{E}-03$ & 25 & & \\
\hline $\mathrm{P}$ & GO:0010467 & gene expression & $1.30 \mathrm{E}-03$ & 39 & & & & & $2.80 \mathrm{E}-03$ & 19 \\
\hline $\mathrm{P}$ & GO:0042592 & homeostatic process & & & & & $2.00 \mathrm{E}-02$ & 10 & & \\
\hline $\mathrm{P}$ & GO:0051179 & localization & $8.70 \mathrm{E}-03$ & 35 & & & $2.30 \mathrm{E}-03$ & 27 & & \\
\hline $\mathrm{P}$ & GO:0009059 & macromolecule biosynthetic process & $2.10 \mathrm{E}-03$ & 36 & & & & & $1.40 \mathrm{E}-03$ & 19 \\
\hline$P$ & GO:0043170 & macromolecule metabolic process & $1.20 \mathrm{E}-05$ & 66 & & & $2.30 \mathrm{E}-03$ & 39 & $1.40 \mathrm{E}-03$ & 28 \\
\hline $\mathrm{P}$ & GO:0043412 & macromolecule modification & $2.20 \mathrm{E}-02$ & 19 & & & & & & \\
\hline $\mathrm{P}$ & GO:0008152 & metabolic process & $2.70 \mathrm{E}-06$ & 86 & $5.20 \mathrm{E}-05$ & 50 & 7.10E-05 & 55 & $1.30 \mathrm{E}-03$ & 37 \\
\hline $\mathrm{P}$ & GO:0007275 & multicellular organismal development & 4.00E-05 & 42 & & & $2.00 \mathrm{E}-02$ & 23 & $1.30 \mathrm{E}-03$ & 20 \\
\hline$P$ & GO:0032501 & multicellular organismal process & $9.20 \mathrm{E}-04$ & 56 & & & 4.80E-02 & 32 & $2.70 \mathrm{E}-02$ & 23 \\
\hline$P$ & GO:0006807 & nitrogen compound metabolic process & $5.00 \mathrm{E}-03$ & 41 & & & & & $6.40 \mathrm{E}-03$ & 20 \\
\hline $\mathrm{P}$ & GO:0006139 & nucleobase, nucleoside, nucleotide and nucleic acid metabolic process & $8.70 \mathrm{E}-03$ & 37 & & & & & $5.20 \mathrm{E}-03$ & 19 \\
\hline $\mathrm{P}$ & GO:0006996 & organelle organization & $2.70 \mathrm{E}-03$ & 20 & & & & & & \\
\hline $\mathrm{P}$ & GO:0044238 & primary metabolic process & $3.50 \mathrm{E}-05$ & 73 & $1.50 \mathrm{E}-04$ & 44 & 3.00E-04 & 48 & $1.40 \mathrm{E}-03$ & 32 \\
\hline
\end{tabular}




\begin{tabular}{|c|c|c|c|c|c|c|c|c|c|c|}
\hline \multirow[b]{2}{*}{ Ontology } & \multirow[b]{2}{*}{ GO Term } & \multirow[b]{2}{*}{ Description } & \multicolumn{2}{|c|}{$\begin{array}{l}\text { Methylated in Sham } \\
\text { tissues }\end{array}$} & \multicolumn{2}{|c|}{$\begin{array}{l}\text { Methylated in } \\
\text { TumDBC tissue }\end{array}$} & \multicolumn{2}{|c|}{$\begin{array}{l}\text { Methylated in } \\
\text { TumBaP tissue }\end{array}$} & \multicolumn{2}{|c|}{$\begin{array}{c}\text { Methylated in } \\
\text { TumDBC+TumBaP } \\
\text { tissues }\end{array}$} \\
\hline & & & FDR & Num & FDR & Num & FDR & Num & FDR & Num \\
\hline $\mathrm{P}$ & GO:0019538 & protein metabolic process & $7.30 \mathrm{E}-03$ & 31 & & & $2.00 \mathrm{E}-02$ & 21 & & \\
\hline$P$ & GO:0006464 & protein modification process & $3.00 \mathrm{E}-02$ & 18 & & & & & & \\
\hline $\mathrm{P}$ & GO:0050789 & regulation of biological process & $5.10 \mathrm{E}-03$ & 64 & & & $4.80 \mathrm{E}-02$ & 39 & & \\
\hline $\mathrm{P}$ & GO:0050794 & regulation of cellular process & 2.30E-02 & 58 & & & & & & \\
\hline $\mathrm{P}$ & GO:0010468 & regulation of gene expression & 4.00E-05 & 36 & & & & & $2.50 \mathrm{E}-03$ & 16 \\
\hline$P$ & GO:0060255 & regulation of macromolecule metabolic process & $2.10 \mathrm{E}-04$ & 37 & & & & & $2.80 \mathrm{E}-03$ & 17 \\
\hline $\mathrm{P}$ & GO:0019222 & regulation of metabolic process & $8.10 \mathrm{E}-04$ & 38 & & & & & $1.40 \mathrm{E}-03$ & 19 \\
\hline$P$ & GO:0000003 & reproduction & & & $2.10 \mathrm{E}-02$ & 9 & & & & \\
\hline$P$ & GO:0006350 & transcription & $2.10 \mathrm{E}-03$ & 29 & & & & & $1.40 \mathrm{E}-03$ & 16 \\
\hline$P$ & GO:0006810 & transport & & & & & $2.10 \mathrm{E}-03$ & 25 & & \\
\hline $\mathrm{F}$ & GO:0005488 & binding & $1.30 \mathrm{E}-12$ & 129 & $2.30 \mathrm{E}-11$ & 77 & $2.20 \mathrm{E}-07$ & 77 & 7.10E-03 & 44 \\
\hline $\mathrm{F}$ & GO:0003677 & DNA binding & $3.10 \mathrm{E}-05$ & 32 & & & & & $1.20 \mathrm{E}-02$ & 13 \\
\hline $\mathrm{F}$ & GO:0046872 & metal ion binding & $1.00 \mathrm{E}-03$ & 42 & $9.80 \mathrm{E}-04$ & 26 & 4.90E-02 & 23 & & \\
\hline $\mathrm{F}$ & GO:0003824 & catalytic activity & $1.00 \mathrm{E}-03$ & 57 & $1.50 \mathrm{E}-05$ & 40 & $1.00 \mathrm{E}-04$ & 42 & & \\
\hline $\mathrm{F}$ & GO:0043169 & cation binding & $1.00 \mathrm{E}-03$ & 42 & $6.50 \mathrm{E}-04$ & 27 & $3.70 \mathrm{E}-02$ & 24 & & \\
\hline $\mathrm{F}$ & GO:0043167 & ion binding & $1.00 \mathrm{E}-03$ & 42 & $3.10 \mathrm{E}-04$ & 28 & $3.70 \mathrm{E}-02$ & 24 & & \\
\hline $\mathrm{F}$ & GO:0005515 & protein binding & $1.00 \mathrm{E}-03$ & 61 & $2.30 \mathrm{E}-02$ & 32 & $4.90 \mathrm{E}-03$ & 39 & & \\
\hline $\mathrm{F}$ & GO:0003676 & nucleic acid binding & $1.00 \mathrm{E}-03$ & 35 & & & & & 7.10E-03 & 17 \\
\hline $\mathrm{F}$ & GO:0005509 & calcium ion binding & $7.70 \mathrm{E}-03$ & 11 & & & & & & \\
\hline $\mathrm{F}$ & GO:0030528 & transcription regulator activity & $1.00 \mathrm{E}-02$ & 18 & & & & & 7.10E-03 & 11 \\
\hline $\mathrm{F}$ & GO:0016787 & hydrolase activity & $1.10 \mathrm{E}-02$ & 28 & $2.00 \mathrm{E}-02$ & 17 & $3.70 \mathrm{E}-02$ & 18 & & \\
\hline $\mathrm{F}$ & GO:0003700 & transcription factor activity & $4.50 \mathrm{E}-02$ & 12 & & & & & & \\
\hline $\mathrm{F}$ & GO:0000166 & nucleotide binding & & & $9.80 \mathrm{E}-04$ & 20 & & & & \\
\hline $\mathrm{F}$ & GO:0016740 & transferase activity & & & 4.00E-03 & 16 & $3.70 \mathrm{E}-02$ & 15 & & \\
\hline $\mathrm{F}$ & GO:0030234 & enzyme regulator activity & & & $2.80 \mathrm{E}-02$ & 8 & $3.70 \mathrm{E}-02$ & 9 & & \\
\hline $\mathrm{F}$ & GO:0008233 & peptidase activity & & & & & $1.30 \mathrm{E}-03$ & 11 & & \\
\hline $\mathrm{F}$ & GO:0016772 & transferase activity, transferring phosphorus-containing groups & & & & & 4.60E-02 & 10 & & \\
\hline $\mathrm{F}$ & GO:0016301 & kinase activity & & & & & 4.90E-02 & 9 & & \\
\hline $\mathrm{F}$ & GO:0005215 & transporter activity & & & & & & & $2.10 \mathrm{E}-02$ & 9 \\
\hline $\mathrm{C}$ & GO:0044464 & cell part & $9.00 \mathrm{E}-18$ & 168 & $5.80 \mathrm{E}-10$ & 89 & $9.90 \mathrm{E}-18$ & 112 & $8.90 \mathrm{E}-09$ & 68 \\
\hline $\mathrm{C}$ & GO:0005623 & cell & $9.00 \mathrm{E}-18$ & 168 & $5.80 \mathrm{E}-10$ & 89 & $9.90 \mathrm{E}-18$ & 112 & $8.90 \mathrm{E}-09$ & 68 \\
\hline $\mathrm{C}$ & GO:0044424 & intracellular part & 3.10E-11 & 118 & $1.10 \mathrm{E}-04$ & 58 & $9.60 \mathrm{E}-10$ & 77 & 2.30E-07 & 51 \\
\hline $\mathrm{C}$ & GO:0005622 & intracellular & $3.10 \mathrm{E}-11$ & 120 & $1.10 \mathrm{E}-04$ & 59 & $3.90 \mathrm{E}-12$ & 83 & $2.20 \mathrm{E}-07$ & 52 \\
\hline $\mathrm{C}$ & GO:0043226 & organelle & 4.60E-08 & 98 & 7.00E-04 & 49 & $2.40 \mathrm{E}-05$ & 59 & $5.10 \mathrm{E}-08$ & 48 \\
\hline $\mathrm{C}$ & GO:0043229 & intracellular organelle & 7.30E-08 & 97 & 7.00E-04 & 49 & $2.40 \mathrm{E}-05$ & 59 & $5.10 \mathrm{E}-08$ & 48 \\
\hline $\mathrm{C}$ & GO:0043231 & intracellular membrane-bounded organelle & 7.50E-06 & 83 & $3.70 \mathrm{E}-04$ & 46 & $1.30 \mathrm{E}-04$ & 52 & $2.30 \mathrm{E}-07$ & 43 \\
\hline
\end{tabular}




\begin{tabular}{|c|c|c|c|c|c|c|c|c|c|c|}
\hline \multirow[b]{2}{*}{ Ontology } & \multirow[b]{2}{*}{ GO Term } & \multirow[b]{2}{*}{ Description } & \multicolumn{2}{|c|}{$\begin{array}{l}\text { Methylated in Sham } \\
\text { tissues }\end{array}$} & \multicolumn{2}{|c|}{$\begin{array}{l}\text { Methylated in } \\
\text { TumDBC tissue }\end{array}$} & \multicolumn{2}{|c|}{$\begin{array}{l}\text { Methylated in } \\
\text { TumBaP tissue }\end{array}$} & \multicolumn{2}{|c|}{$\begin{array}{l}\text { Methylated in } \\
\text { TumDBC+TumBaP } \\
\text { tissues }\end{array}$} \\
\hline & & & FDR & Num & FDR & Num & FDR & Num & FDR & Num \\
\hline $\mathrm{C}$ & GO:0043227 & membrane-bounded organelle & $7.50 \mathrm{E}-06$ & 83 & $3.70 \mathrm{E}-04$ & 46 & $1.30 \mathrm{E}-04$ & 52 & $2.30 \mathrm{E}-07$ & 43 \\
\hline $\mathrm{C}$ & GO:0005737 & cytoplasm & $7.60 \mathrm{E}-06$ & 79 & $1.30 \mathrm{E}-03$ & 42 & $4.10 \mathrm{E}-09$ & 61 & $7.40 \mathrm{E}-03$ & 31 \\
\hline $\mathrm{C}$ & GO:0005576 & extracellular region & $1.10 \mathrm{E}-05$ & 31 & $3.20 \mathrm{E}-03$ & 16 & & & & \\
\hline $\mathrm{C}$ & GO:0005634 & nucleus & $3.00 \mathrm{E}-05$ & 55 & $1.50 \mathrm{E}-02$ & 27 & & & 7.90E-05 & 27 \\
\hline $\mathrm{C}$ & GO:0044446 & intracellular organelle part & $1.20 \mathrm{E}-03$ & 36 & $4.70 \mathrm{E}-02$ & 18 & & & & \\
\hline $\mathrm{C}$ & GO:0044422 & organelle part & $1.40 \mathrm{E}-03$ & 36 & $4.90 \mathrm{E}-02$ & 18 & & & & \\
\hline $\mathrm{C}$ & GO:0016020 & membrane & $1.50 \mathrm{E}-03$ & 76 & $1.10 \mathrm{E}-04$ & 49 & $2.10 \mathrm{E}-07$ & 61 & $1.80 \mathrm{E}-02$ & 32 \\
\hline $\mathrm{C}$ & GO:0043232 & intracellular non-membrane-bounded organelle & $1.70 \mathrm{E}-03$ & 27 & & & & & & \\
\hline $\mathrm{C}$ & GO:0043228 & non-membrane-bounded organelle & $1.70 \mathrm{E}-03$ & 27 & & & & & & \\
\hline $\mathrm{C}$ & GO:0005615 & extracellular space & $3.10 \mathrm{E}-03$ & 13 & & & & & & \\
\hline $\mathrm{C}$ & GO:0044444 & cytoplasmic part & $6.90 \mathrm{E}-03$ & 46 & $1.30 \mathrm{E}-02$ & 27 & $2.40 \mathrm{E}-05$ & 38 & $1.80 \mathrm{E}-02$ & 21 \\
\hline $\mathrm{C}$ & GO:0005694 & chromosome & $7.00 \mathrm{E}-03$ & 10 & & & & & & \\
\hline $\mathrm{C}$ & GO:0044421 & extracellular region part & $9.60 \mathrm{E}-03$ & 15 & & & & & & \\
\hline $\mathrm{C}$ & GO:0032991 & macromolecular complex & $1.30 \mathrm{E}-02$ & 31 & & & & & & \\
\hline $\mathrm{C}$ & GO:0005856 & cytoskeleton & $4.60 \mathrm{E}-02$ & 16 & & & & & & \\
\hline $\mathrm{C}$ & GO:0005886 & plasma membrane & & & $2.90 \mathrm{E}-02$ & 20 & $6.10 \mathrm{E}-04$ & 27 & & \\
\hline $\mathrm{C}$ & GO:0005794 & Golgi apparatus & & & $3.50 \mathrm{E}-02$ & 8 & & & & \\
\hline $\mathrm{C}$ & GO:0016023 & cytoplasmic membrane-bounded vesicle & & & & & $1.50 \mathrm{E}-03$ & 8 & & \\
\hline $\mathrm{C}$ & GO:0031988 & membrane-bounded vesicle & & & & & $2.20 \mathrm{E}-03$ & 8 & & \\
\hline $\mathrm{C}$ & GO:0031410 & cytoplasmic vesicle & & & & & $3.50 \mathrm{E}-03$ & 9 & & \\
\hline $\mathrm{C}$ & GO:0005829 & cytosol & & & & & $3.60 \mathrm{E}-03$ & 9 & & \\
\hline $\mathrm{C}$ & GO:0031982 & vesicle & & & & & $5.20 \mathrm{E}-03$ & 9 & & \\
\hline
\end{tabular}

Note: Gene lists for functional ontology analysis were generated by selecting features with $\log _{2}$ peak ratio $>2$ (indicates methylated DNA) in all three samples of interest and excluding all features that were not methylated $\left(\log _{2}\right.$ peak ratio $\left.<2\right)$ in at least 2 of the 3 biological samples not of interest. Thus, genes in the methylated Sham list were consistently methylated in only the sham tissues. Gene ontology was performed using AgriGO (Du, et al., 2010) using the singular enrichment analysis tool against the mouse gene ontology database (Mouse Genome Informatics) with the following

parameters: Fisher test with FDR under dependency correction and significance level of $P<0.05$ and the minimum number of mapping entries set at 5 genes. The gene ontology type performed was a generic GO slim (Gene Ontology Consortium). GO terms shown above are for biological processes (B), molecular function (F) and cellular component (C). For each tissue, the FDR-adjusted $P$ value and the number of genes associated with the GO term are shown. Color blocks indicate the level of significance for each GO term, color-coded according to the tissue type for cross reference with Figures and supplemental materials (gray scale, Sham; red scale, TumDBC; orange scale, TumBaP; light blue scale, union set for both TumDBC and TumBaP). Absence of $P$ value and gene numbers for a GO term indicates that that term was not significantly enriched for that particular gene set. For clarity, in cases where a biological process is represented in more than one tissue type, the specific methylated genes associated with that term are different for each tissue. 
Appendix F

Gene Ontology Maps

Gene ontology maps were generated for all group comparisons outlined in Table D.1.; these maps are provided as high resolution .tif files at the DOI link available in the following reference (Fish, 2015e). This archive includes 10 individual image files (see Table F.1), each of which depicts a Gene Ontolgoy map for GO terms representing hypermethylated gene promoters unique for the indicated tissue(s) (listed first in file name) compared to other tissues. Gene ontology analysis was performed using the AgriGO singular enrichment analysis (SEA) tool (http://bioinfo.cau.edu.cn/agriGO) against the mouse gene ontology database (Mouse Genome Informatics). Significantly enriched GO terms (Generic GO Slim) were determined using the Fisher test with an FDR-corrected significance level of $P<0.05$ (Yekutieli multi-test adjustment method) and the minimum number of mapping entries set at 5 genes.

\section{TABLE F.1}

\section{List of File Names Included in the Archive File for Appendix F}

Figure designation File name

Fig. F.1

Fig. F.2

Fig. F.3

Fig. F.4

Fig. F.5

Fig. F.6

Fig. F.7

Fig. F.8

Fig. F.9

Fig. F.10
GO Slim Map for Methylated in Sham, Not AdjDBC or TumDBC.tif GO Slim Map for Methylated in AdjDBC, Not Sham or TumDBC.tif GO Slim Map for Methylated in TumDBC, Not Sham or AdjDBC.tif GO Slim Map for Methylated in Sham, Not AdjBaP or TumBaP.tif GO Slim Map for Methylated in AdjBaP, Not Sham or TumBaP.tif GO Slim Map for Methylated in TumBaP, Not Sham or AdjBaP.tif GO Slim Map for Methylated in Sham, Not TumDBC or TumBaP.tif GO Slim Map for Methylated in TumDBC, Not Sham or TumBaP.tif GO Slim Map for Methylated in TumBaP, Not Sham or TumDBC.tif GO Slim Map for Methylated in TumDBC and TumBaP, Not Sham.tif 